GREGOR KONRAD ENNES SIMONS

ENGENHEIRO CIVIL - ESCOLA POLITÉCNICA DA USP

2002

UMA INVESTIGAÇÃO SOBRE A APLICABILIDADE DA TEORIA DE SISTEMAS NÃO-IDEAIS A FUNDAÇÕES DE MÁQUINAS REAIS

Dissertação apresentada à Escola Politécnica da Universidade de São Paulo para obtenção do título de Mestre em Engenharia 
GREGOR KONRAD ENNES SIMONS

ENGENHEIRO CIVIL - ESCOLA POLITÉCNICA DA USP

2002

\section{UMA INVESTIGAÇÃO SOBRE A APLICABILIDADE DA TEORIA DE SISTEMAS NÃO-IDEAIS A FUNDAÇÕES DE MÁQUINAS REAIS}

Dissertação apresentada à Escola Politécnica da Universidade de São Paulo para obtenção do título de Mestre em Engenharia

Área de Concentração:

Engenharia de Estruturas

Orientador:

Prof. Livre-Docente

Reyolando Manoel Lopes Rebello da

Fonseca Brasil 
FICHA CATALOGRÁFICA

Simons, Gregor Konrad Ennes

Uma investigação sobre a aplicabilidade da teoria de sistemas não-ideais a fundações de máquinas reais / G.K.E. Simons. -- São Paulo, 2008.

$135 \mathrm{p}$.

Dissertação (Mestrado) - Escola Politécnica da Universidade de São Paulo. Departamento de Engenharia de Estruturas e Geotécnica.

1. Dinâmica das estruturas 2. Efeito Sommerfeld 3. Sistema ideal e não-ideal de energia 4. Fundações de máquinas I. Universidade de São Paulo. Escola Politécnica. Departamento de Engenharia de Estruturas e Geotécnica II. t. 
Aos meus pais, George e Marisa Simons e irmãos, Klaus Christoph e George Aleksandr, que deixa muita saudade. 


\section{AGRADECIMENTOS}

A Deus pela vida.

Ao amigo e orientador Prof. Dr. Reyolando Brasil, pela presença, paciência e constante estímulo transmitido durante todo o trabalho.

Aos meus pais e irmão, simplesmente por existirem e estarem sempre presentes na minha vida, em todas as circunstâncias.

Em especial a Daniela Lourenço, pela paciência e compreensão durante todos esses anos de dedicação.

Ao amigo e gerente da Engenharia Civil da CNEC Engenharia, Luís Fernando Favaro, pelo incentivo e constante apoio.

A WEG Equipamentos Elétricos S/A - Motores, pelas informações fornecidas. 


\section{RESUMO}

O principal objetivo do presente projeto é verificar a interação entre a estrutura da fundação de uma máquina - um ventilador industrial - com a fonte de excitação, no caso seu próprio motor, com o intuito de identificar possível ocorrência do chamado "fenômeno do salto", também conhecido como Efeito Sommerfeld. Tal fenômeno é decorrente do acoplamento dinâmico entre a fonte excitadora e a estrutura durante a passagem pelos diferentes estados de ressonância, à medida que o motor é acelerado, até chegar ao regime permanente, sendo possível observar-se o mesmo fenômeno também no momento da desaceleração. Conceitua-se, neste trabalho, o modelo fonte não-ideal de energia como aquele que decorre da efetiva interação do motor da máquina com a estrutura, diferentemente do modelo de fonte ideal, aqui compreendida como sendo isenta de qualquer tipo de interação com a estrutura. Em razão do modelo adotado, a estrutura em estudo apresenta poucos graus de liberdade, definem-se as equações do movimento, tanto para o sistema ideal, como para o não-ideal, por meio da formulação de Lagrange, e obtêm-se as soluções numéricas. Conclui-se que se as Normas usuais de projeto de fundações de máquinas são empregadas em casos reais da engenharia, os fenômenos não-ideais procurados não são observados.

Palavras-Chave: Efeito Sommerfeld. Fonte ideal e não-ideal de energia. 


\begin{abstract}
The main objective of the present project is to verify the interaction between the foundation structure of a machine - an industrial fan - with the excitation source, its own motor, in this case to identify possible occurrence of the so called "jump phenomenon", also known as Sommerfeld Effect. Such phenomenon is derived from the dynamic coupling between the excitation source and the structure during the passage through the different resonance states as the motor is accelerated until it arrives to its steady-state. It is possible to observe the same phenomenon at the moment of the deceleration. It is considered, in this work, the non-ideal source of energy model as the one that derives from the effective interaction between the motor of the machine with the structure, differently of the ideal source model, herein understood as exempted of any kind of interaction with the structure. As a consequence of the adopted model of the structure under analysis presenting few degrees of freedom, the equations of motion are defined, both for ideal and for nonideal systems, through the formulation of Lagrange, and their numerical solutions are obtained. It is concluded that if usual design Codes for machine foundations are applied to real engineering cases, the searched for phenomena are not observed.
\end{abstract}

Keywords: Sommerfeld effect. Ideal and non-ideal energy source 


\section{LISTA DE FIGURAS}

Figura 1.1 - Arranjo mecânico de turbina a vapor 16

Figura 1.2 - Estrutura tipo aporticada de turbina a vapor .17

Figura 1.3 - Arranjo mecânico de ventilador centrífugo .........................................17

Figura 1.4 - Estrutura tipo bloco de ventilador centrífugo ........................................ 18

Figura 2.1 - Curva de energia consumida $E\left(\omega_{m}\right)$ pela estrutura ............................28

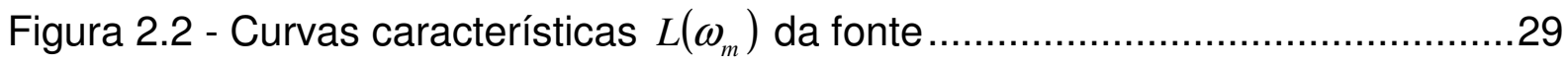

Figura 2.3 - Curvas dos conjugados resistentes internos $H\left(\omega_{m}\right)$ da fonte...............29

Figura 2.4 - Ponto de estabilidade de sistema não-ideal........................................30

Figura 2.5 - Modelo físico experimental de Kononenko e Korablev ..........................31

Figura 2.6 - Curvas características $L\left(\omega_{m}\right)$ do motor e curva $S\left(\omega_{m}\right) \ldots \ldots \ldots \ldots \ldots \ldots \ldots \ldots . . . .31$

Figura 2.7 - Curvas teóricas e experimentais obtidas por Kononenko e Korablev ....32

Figura 2.8 - Comparação entre sistemas ideal e não-ideal .........................................33

Figura 3.1 - Universo tecnológico de motores elétricos..........................................36

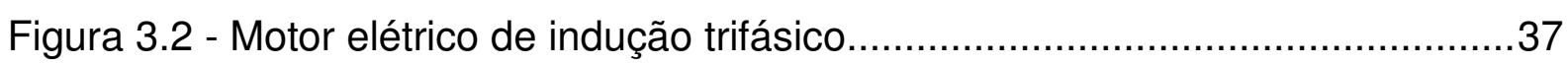

Figura 3.3 - Partes integrantes de motor elétrico …….......................................38

Figura 3.4 - Conjugados do motor e resistente da carga .........................................39

Figura 3.5 - Curvas de conjugado x Velocidade dos diferentes tipos de motor..........39

Figura 3.6 - Curvas de conjugado $x$ Velocidade das diferentes categorias ................40

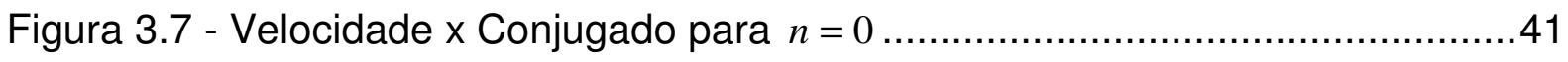

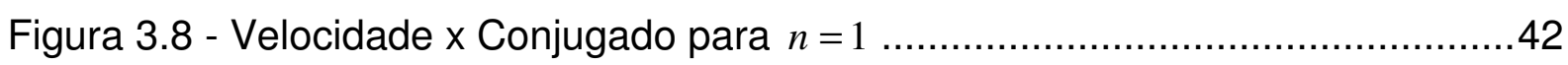

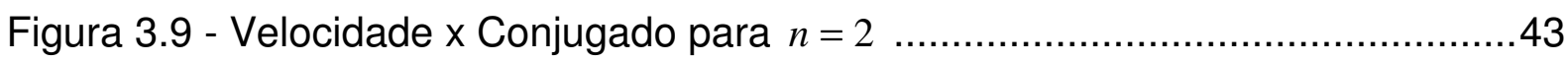

Figura 4.1 - Modelo para formulação: sistema ideal e não-ideal ..............................44

Figura 5.1 - Arranjo simplificado do sistema motor-estrutura ..............................57

Figura 5.2 - Seção longitudinal de uma torre de resfriamento de água......................58

Figura 5.3 - Seção transversal de uma torre de resfriamento de água ......................58 


\section{LISTA DE GRÁFICOS}

Gráfico 6.1 - Deslocamentos: sistema ideal e não-ideal com $r \cdot \omega_{m}=6,3 \mathrm{~mm} / \mathrm{s}$ 61

Gráfico 6.2 - Velocidades: sistema ideal e não-ideal com $r \cdot \omega_{m}=6,3 \mathrm{~mm} / \mathrm{s}$ 61

Gráfico 6.3 - Rotação do motor: sistema não-ideal com $r \cdot \omega_{m}=6,3 \mathrm{~mm} / \mathrm{s}$. 62

Gráfico 6.4 - Planos de fase: sistema ideal e não-ideal com $r \cdot \omega_{m}=6,3 \mathrm{~mm} / \mathrm{s}$ 62

Gráfico 6.5 - Energia consumida pela estrutura x Altura .63

Gráfico 6.6 - Freqüência natural x Altura \& Rotação .63

Gráfico 6.7 - Deslocamento da estrutura x Altura. 63

Gráfico 6.8 - Rotação do motor x Altura 63

Gráfico 6.9 - Plano de fase para $L=1.5 \mathrm{~m}$. .64

Gráfico 6.10 - Plano de fase para $L=1.5 \mathrm{~m}$ detalhe $v \mathrm{l}_{0} \times$ vo. .64

Gráfico 6.11 - Plano de fase para $L=2.0 \mathrm{~m}$. 64

Gráfico 6.12 - Plano de fase para $L=2.0 \mathrm{~m}$ detalhe vel $_{0} \mathrm{x}$ vo .64

Gráfico 6.13 - Plano de fase para $L=2.5 \mathrm{~m}$. .64

Gráfico 6.14 - Plano de fase para $L=2.5 \mathrm{~m}$ detalhe vel $_{0} \times$ vo .64

Gráfico 6.15 - Plano de fase para $L=3.0 \mathrm{~m}$. .64

Gráfico 6.16 - Plano de fase para $L=3.0 \mathrm{~m}$ detalhe vel $_{0} \times$ vo .64

Gráfico 6.17 - Plano de fase para $L=3.5 \mathrm{~m}$. .65

Gráfico 6.18 - Plano de fase para $L=3.5 \mathrm{~m}$ detalhe vel $_{0} \mathrm{x}$ vo .65

Gráfico 6.19 - Plano de fase para $L=4.0 \mathrm{~m}$. 65

Gráfico 6.20 - Plano de fase para $L=4.0 \mathrm{~m}$ detalhe vel $_{0} \mathrm{x}$ vo 65

Gráfico 6.21 - Plano de fase para $L=4.5 \mathrm{~m}$. 65

Gráfico 6.22 - Plano de fase para $L=4.5 \mathrm{~m}$ detalhe vel $_{0} \times$ vo 65

Gráfico 6.23 - Plano de fase para $L=5.0 \mathrm{~m}$. 65

Gráfico 6.24 - Plano de fase para $L=5.0 \mathrm{~m}$ detalhe $v \mathrm{l}_{0} \times$ vo. .65

Gráfico 6.25 - Plano de fase para $L=5.5 \mathrm{~m}$. .66

Gráfico 6.26 - Plano de fase para $L=5.5 \mathrm{~m}$ detalhe vel $_{0} \mathrm{x}$ vo .66

Gráfico 6.27 - Plano de fase para $L=6.0 \mathrm{~m}$. .66

Gráfico 6.28 - Plano de fase para $L=6.0 \mathrm{~m}$ detalhe vel $_{0} \times$ vo .66

Gráfico 6.29 - Energia consumida pela estrutura x Altura .67

Gráfico 6.30 - Freqüência natural x Altura \& Rotação 


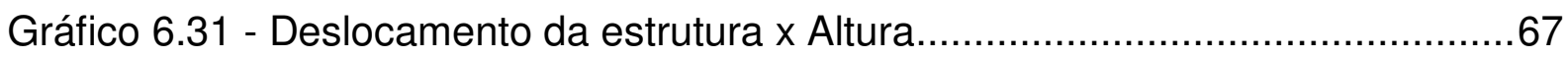

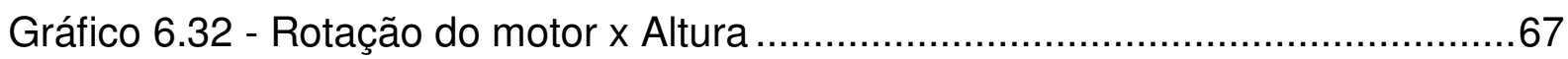

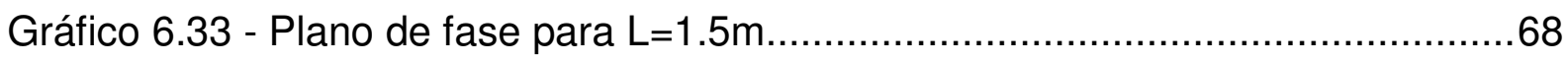

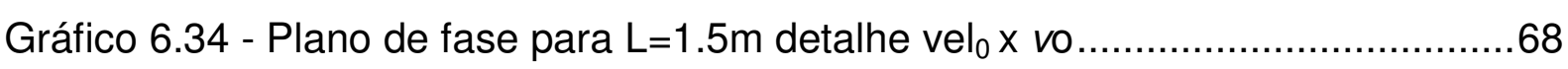

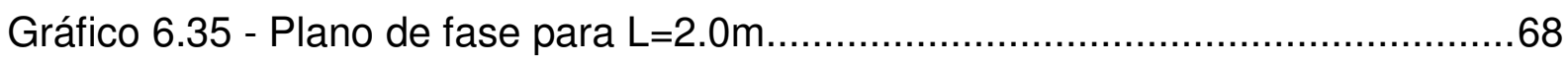

Gráfico 6.36 - Plano de fase para $L=2.0 \mathrm{~m}$ detalhe $v^{2} \mathrm{l}_{0} \mathrm{x}$ vo .................................68

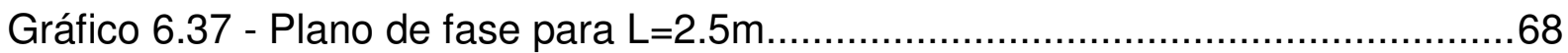

Gráfico 6.38 - Plano de fase para $L=2.5 \mathrm{~m}$ detalhe $v^{2} \mathrm{l}_{0} \times$ vo ..................................68

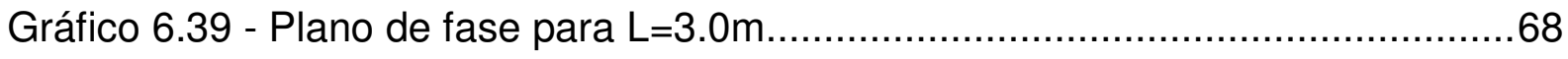

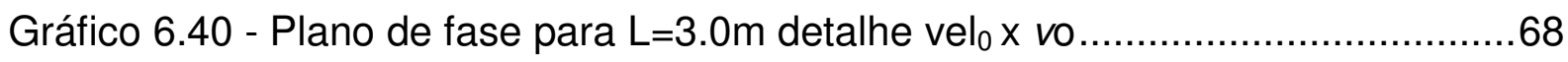

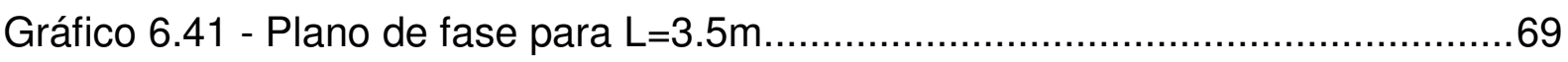

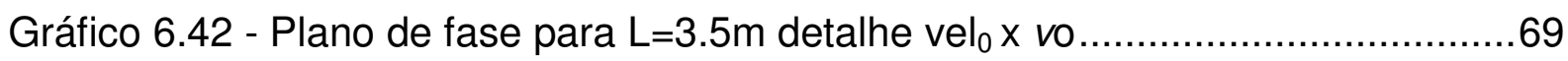

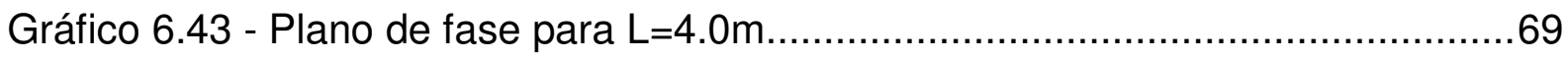

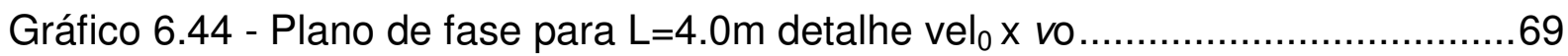

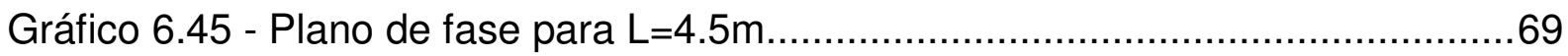

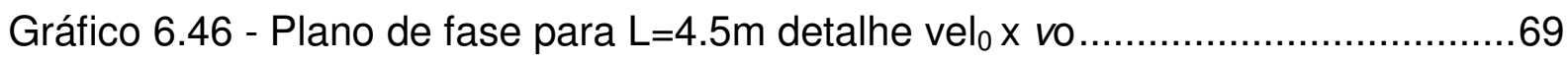

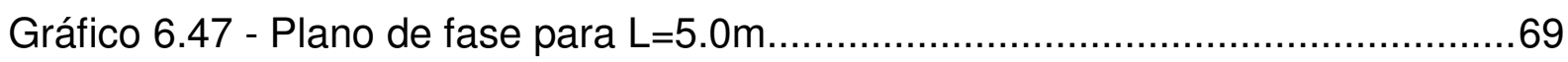

Gráfico 6.48 - Plano de fase para $L=5.0 \mathrm{~m}$ detalhe $v^{2} \mathrm{l}_{0} \mathrm{x}$ vo ................................69

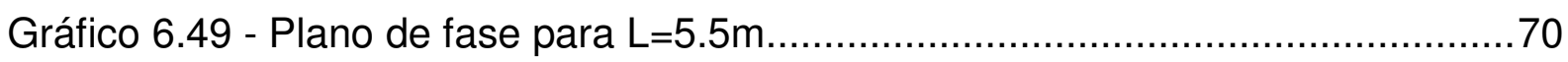

Gráfico 6.50 - Plano de fase para $L=5.5 \mathrm{~m}$ detalhe $v^{2} \mathrm{l}_{0} \mathrm{x}$ vo ..................................

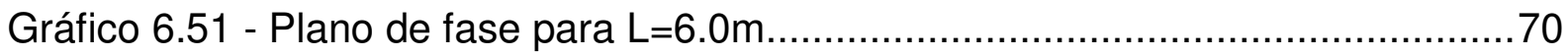

Gráfico 6.52 - Plano de fase para $L=6.0 \mathrm{~m}$ detalhe vel $_{0} \times$ vo ….............................70

Gráfico 6.53 - Energia consumida pela estrutura x Altura ......................................71

Gráfico 6.54 - Freqüência natural x Altura \& Rotação ...............................................71

Gráfico 6.55 - Deslocamento da estrutura x Altura................................................71

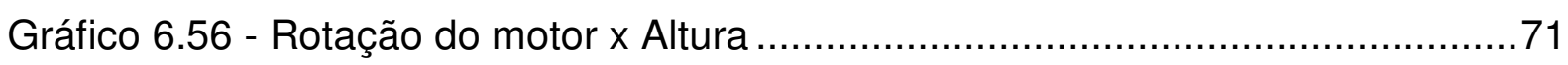

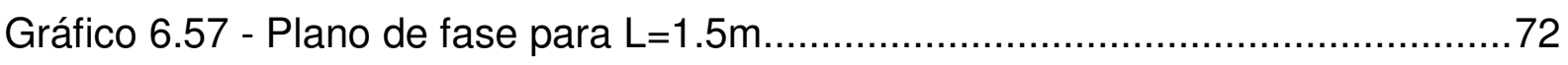

Gráfico 6.58 - Plano de fase para $\mathrm{L}=1.5 \mathrm{~m}$ detalhe vel $\mathrm{x}$ vo ...................................72

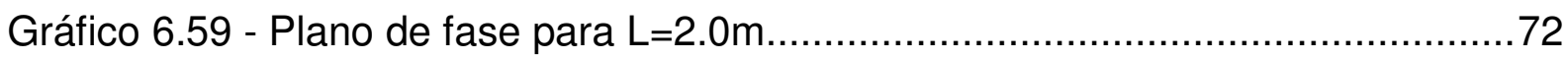

Gráfico 6.60 - Plano de fase para $L=2.0 \mathrm{~m}$ detalhe vel $\mathrm{x}$ vo ..................................72

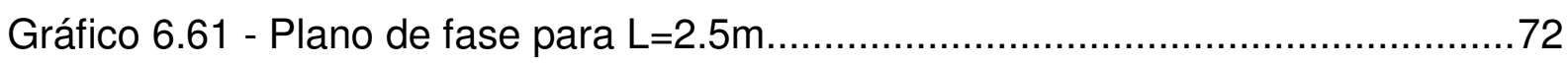

Gráfico 6.62 - Plano de fase para $L=2.5 \mathrm{~m}$ detalhe vel $_{0} \times \mathrm{vo}$..................................

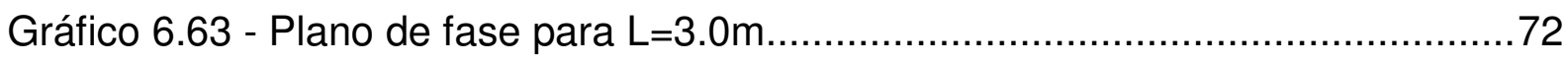

Gráfico 6.64 - Plano de fase para $L=3.0 \mathrm{~m}$ detalhe $v^{2} \mathrm{l}_{0} \mathrm{x}$ vo .................................72 


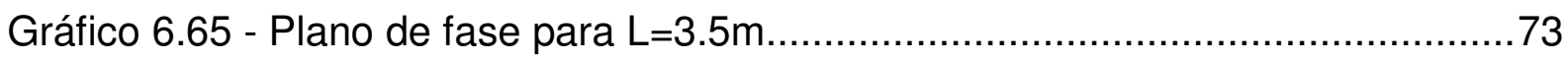

Gráfico 6.66 - Plano de fase para $L=3.5 \mathrm{~m}$ detalhe vel $_{0} \mathrm{x}$ vo ....................................

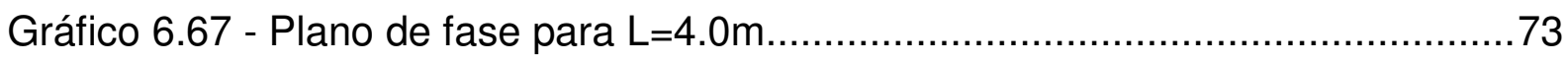

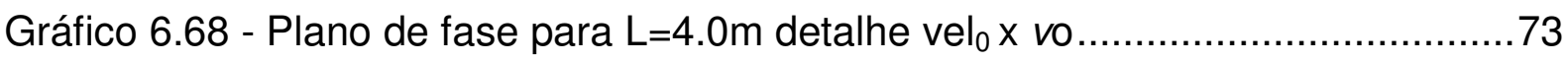

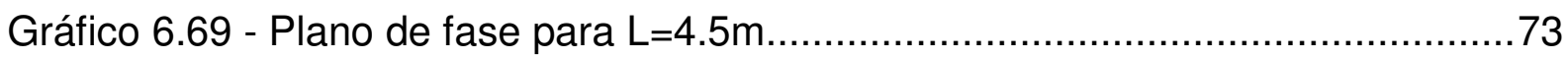

Gráfico 6.70 - Plano de fase para $L=4.5 \mathrm{~m}$ detalhe vel $_{0} \mathrm{x}$ vo .................................73

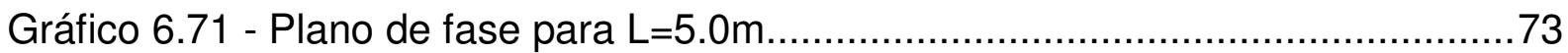

Gráfico 6.72 - Plano de fase para $L=5.0 \mathrm{~m}$ detalhe $v^{2} \mathrm{l}_{0} \mathrm{x}$ vo ................................73

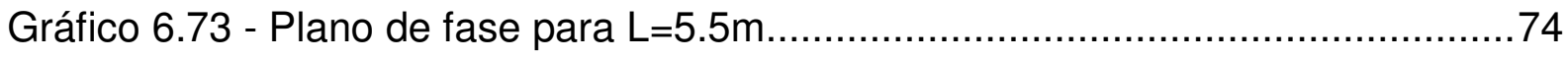

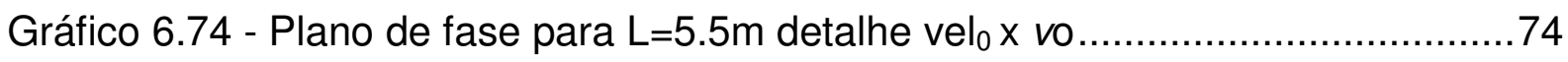

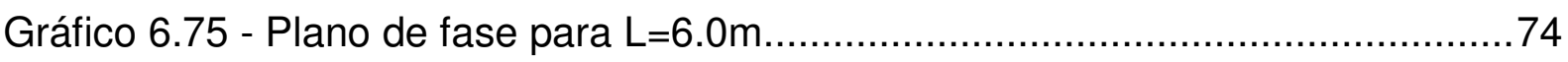

Gráfico 6.76 - Plano de fase para $L=6.0 \mathrm{~m}$ detalhe vel $_{0} \mathrm{x}$ vo ................................74

Gráfico 6.77 - Energia consumida E0 x Altura (r0.w0m: 6.3mm/s).......................75

Gráfico 6.78 - Energia consumida E1 x Altura (r1.w1m: 2000mm/s)....................75

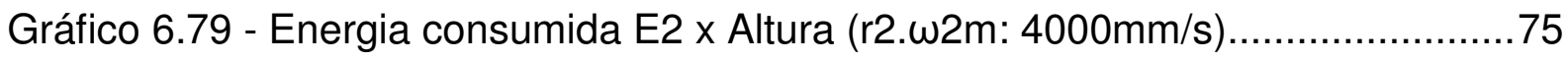

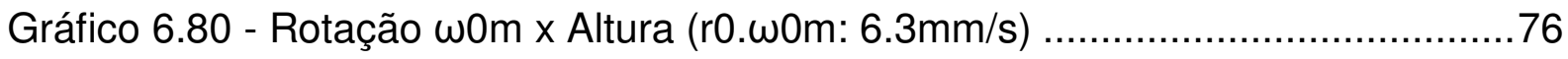

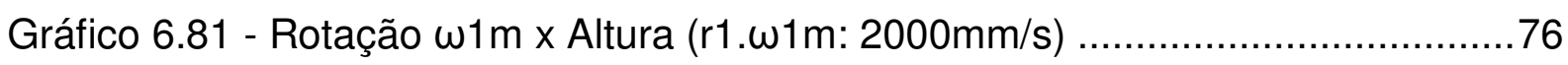

Gráfico 6.82 - Rotação w2m x Altura (r2.w2m: 4000mm/s) ................................76

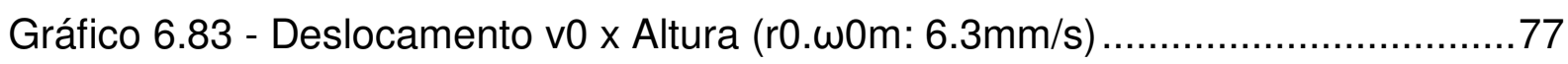

Gráfico 6.84 - Deslocamento v1 x Altura (r1.w1 m: 2000mm/s) ….......................77

Gráfico 6.85 - Deslocamento v2 x Altura (r2.w2m: 4000mm/s) …........................77

Gráfico 7.1 - Comparação entre rigidez à flexão e geométrica x Altura ....................80

Gráfico 7.2 - Influência da rigidez geométrica x Altura .............................................80 


\section{LISTA DE TABELAS}

Tabela 3.1 - Velocidades síncronas .43

Tabela 6.1 - Respostas do sistema não-ideal $b=0.75, h=0.80$ e $L=$ variável $(m) \ldots . .63$

Tabela 6.2 - Respostas do sistema não-ideal $b=0.75, h=1.00$ e $L=$ variável $(m) \ldots . .67$

Tabela 6.3 - Respostas do sistema não-ideal $b=0.95, h=0.80$ e $L=$ variável $(m) \ldots . .71$ 


\section{LISTA DE SÍMBOLOS}

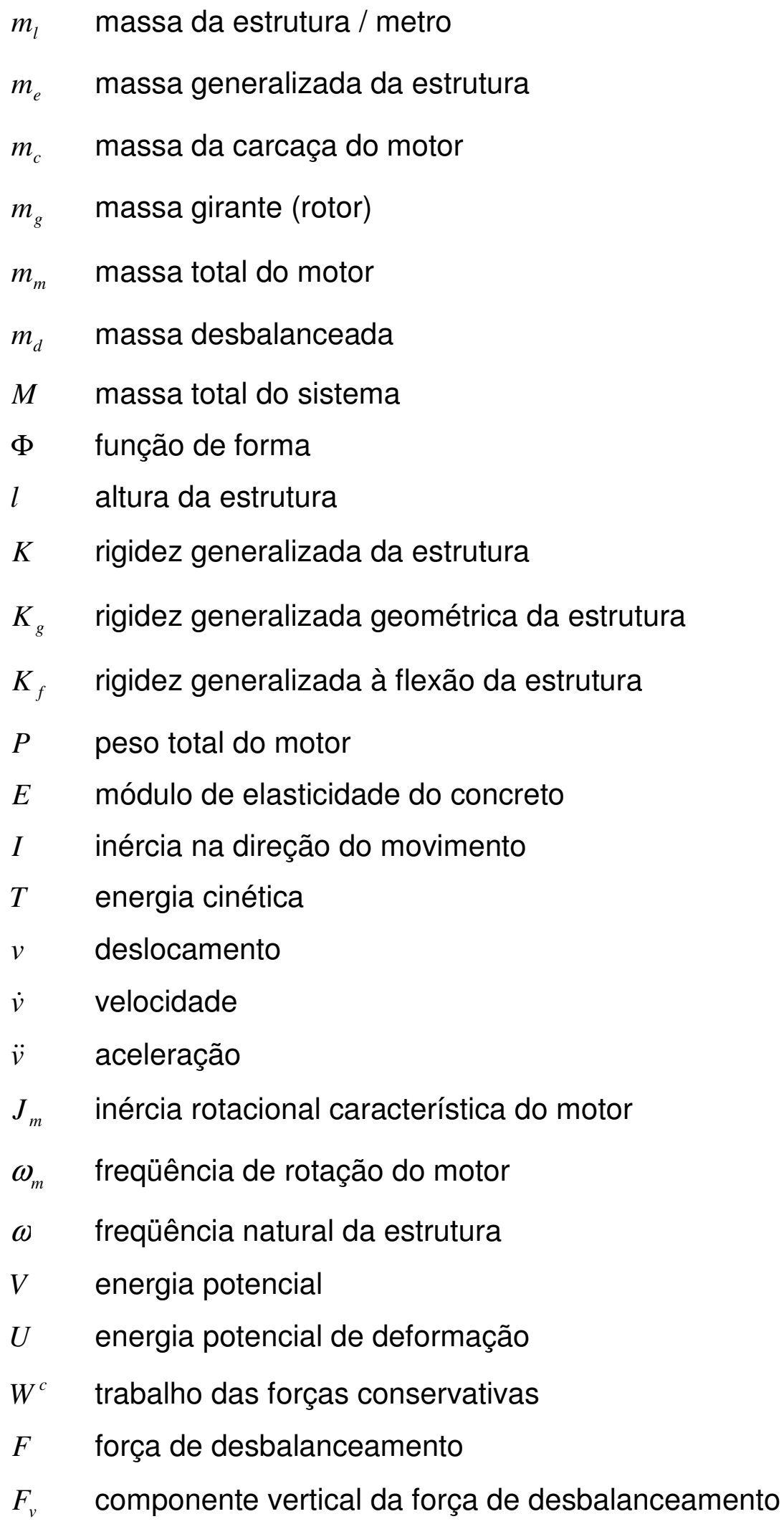




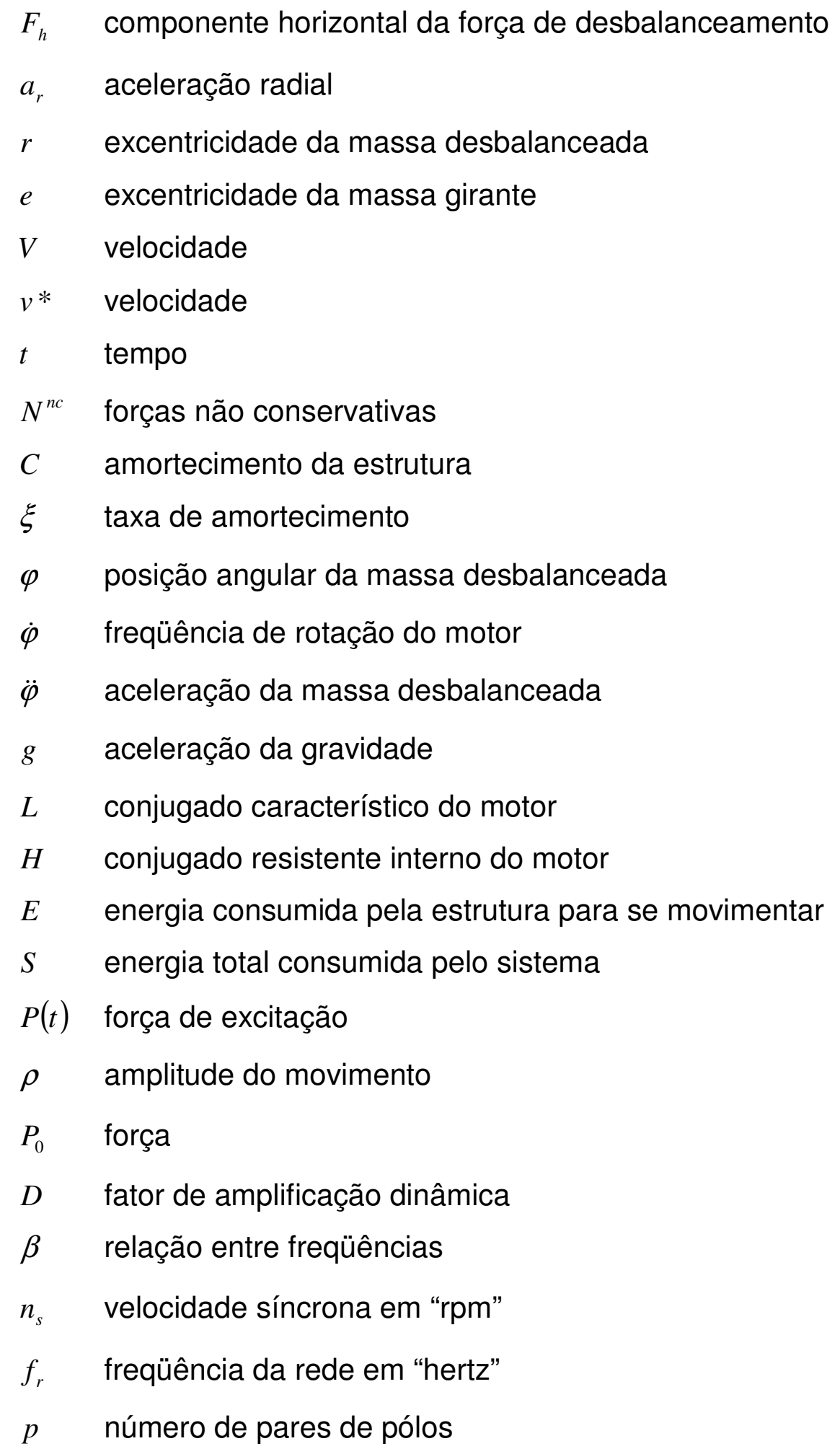




\section{SUMÁRIO}

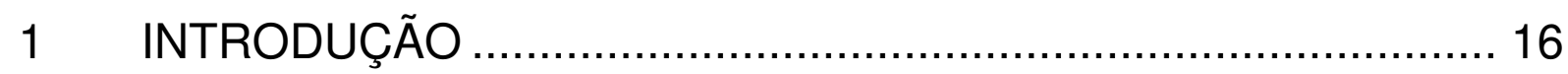

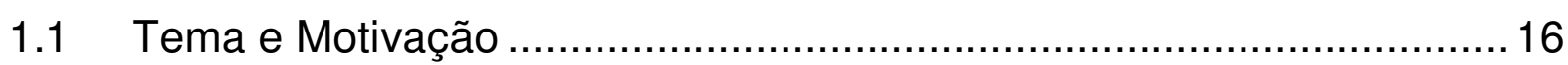

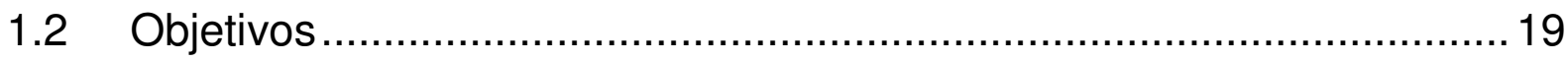

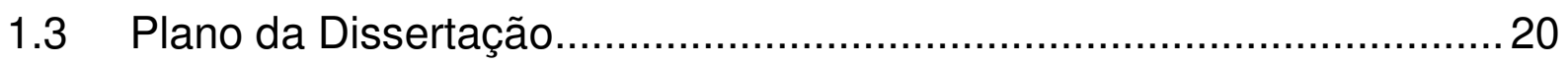

2 REVISÃO DA LITERATURA ............................................ 21

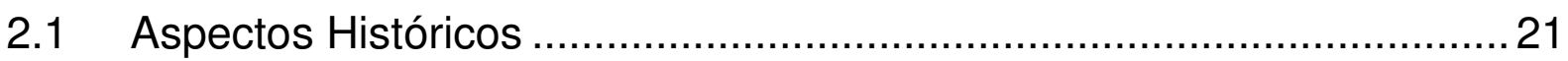

2.2 Diferença entre os Modelos de Fonte de Energia Ideal e Não-Ideal .... 25

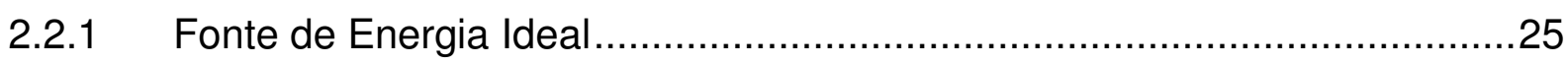

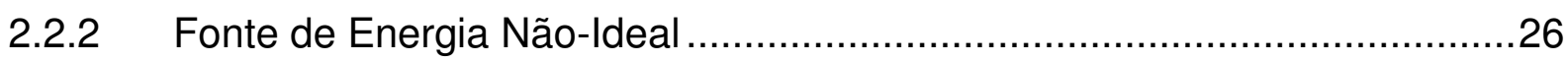

2.3 Características das Fontes de Energia .............................................. 28

2.4 Estabilidade de Sistema com Fonte de Energia Não-Ideal ................... 29

2.5 Fenômenos Relacionados à Fonte Não-Ideal ..................................... 30

3 NOÇÕES FUNDAMENTAIS SOBRE MOTORES ..................... 34

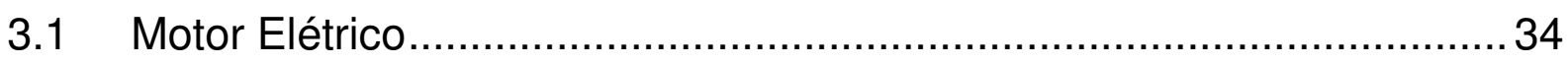

3.1.1 Motores Elétricos de Corrente Contínua ..................................................35

3.1.2 Motores Elétricos de Corrente Alternada ……..........................................35

3.2 Curvas dos Conjugados Motor e Resistente da Carga .........................39

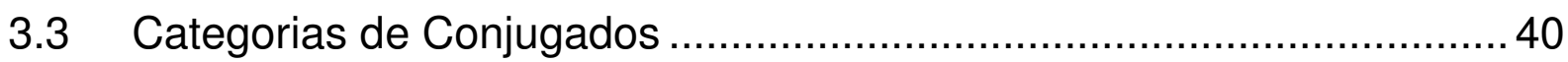

3.4 Curvas Características Conjugado / Veloc. das Cargas Mecânicas ..... 41

3.4.1 Cargas de Conjugado Resistente Constante $(n=0) \ldots \ldots \ldots \ldots \ldots \ldots \ldots \ldots \ldots \ldots . . . .41$

3.4.2 Cargas de Conjugado Resistente Linear com a Velocidade $(n=1)$............42

3.4.3 Cargas de Conjugado Resistente Cresc. com Quadrado da Vel. $(n=2) \ldots . .42$

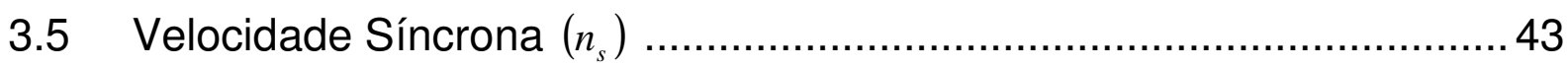

4 FORMULAÇÃO: SISTEMA COM FONTE IDEAL E NÃO-IDEAL DE ENERGIA ............................................................................... 44

4.1 Sistema com Fonte Ideal de Energia ............................................ 44

4.1.1 Cálculo das Características Dinâmicas Generalizadas da Estrutura ..........44

4.1.1.1 Cálculo da Massa Generalizada da Estrutura $m_{e}$ 
4.1.1.2 Cálculo da Rigidez Generalizada da Estrutura $K$.....................46

4.1.2 Cálculo da Energia Cinética do Sistema ..............................................47

4.1.3 Cálculo da Energia Potencial do Sistema ..........................................48

4.1.4 Cálculo da Força não Conservativa ..............................................48

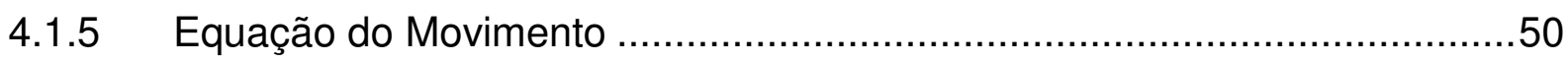

4.2 Sistema com Fonte Não-Ideal de Energia................................... 51

4.2.1 Cálculo da Energia Cinética do Sistema ...........................................51

4.2.2 Cálculo da Energia Potencial do Sistema ...........................................52

4.2.3 Cálculo da Força não Conservativa ....................................................53

4.2.4 Equação do Movimento .........................................................54

5 MATERIAIS E METODOLOGIA....................................... 57

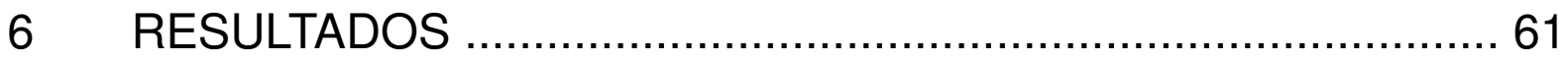

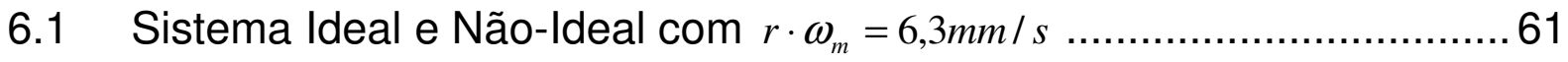

6.2 Sistemas Não-Ideais com $r \cdot \omega_{m}=6,3 \mathrm{~mm} / \mathrm{s}, 2000 \mathrm{~mm} / \mathrm{s}$ e $4000 \mathrm{~mm} / \mathrm{s} \ldots . .62$

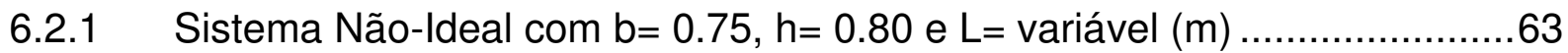

6.2.2 Sistema Não-Ideal com $b=0.75, h=1.00$ e $L=$ variável $(m)$....................67

6.2.3 Sistema Não-Ideal com $b=0.95, h=0.80$ e $\mathrm{L}=$ variável $(\mathrm{m})$.....................71

6.3 Sistemas Não-Ideais com $r \cdot \omega_{m}=6,3 \mathrm{~mm} / \mathrm{s}, 2000 \mathrm{~mm} / \mathrm{s}$ e $4000 \mathrm{~mm} / \mathrm{s}$ para

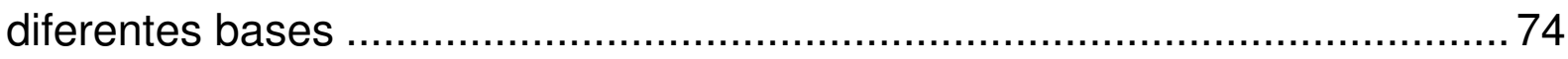

7 DISCUSSÃO DOS RESULTADOS ....................................... 78

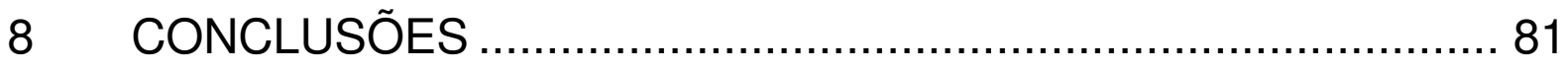

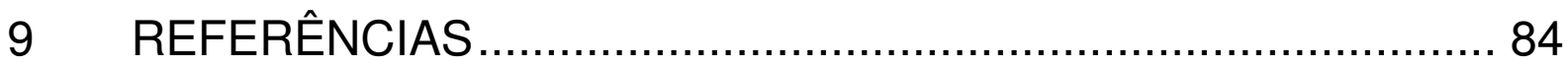

10 REFERÊNCIAS COMPLEMENTARES .............................. 86 APÊNDICE A - ROTINA PARA REGRESSÃO POLINOMIAL ............... 87 APÊNDICE B - ROTINA DE CÁLCULO SISTEMA IDEAL - 1GL.......... 91 APÊNDICE C - ROTINA DE CÁLCULO SISTEMA NÃO-IDEAL - 2GL . 95 APÊNDICE D - GRÁFICOS DAS RESPOSTAS DA ESTRUTURA PARA SISTEMA NÃO-IDEAL DE ENERGIA ........................................ 102 ANEXO A - CARACTERÍSTICAS MECÂNICAS DO MOTOR WEG.....133 


\section{INTRODUÇÃO}

\subsection{Tema e Motivação}

O campo da Engenharia Industrial é constituído basicamente por equipamentos, a maior parte máquinas rotativas, que podem apresentar custos extremamente elevados e estruturas com alta complexidade, como é o caso da fundação de uma turbina a vapor ou a gás. Daí advém a necessidade do desenvolvimento de projetos teoricamente bem fundamentados para limitar as amplitudes de deslocamento de cada tipo de máquina a valores aceitáveis, de modo a não comprometer seu funcionamento e a garantir uma vida útil maior para o sistema como um todo.

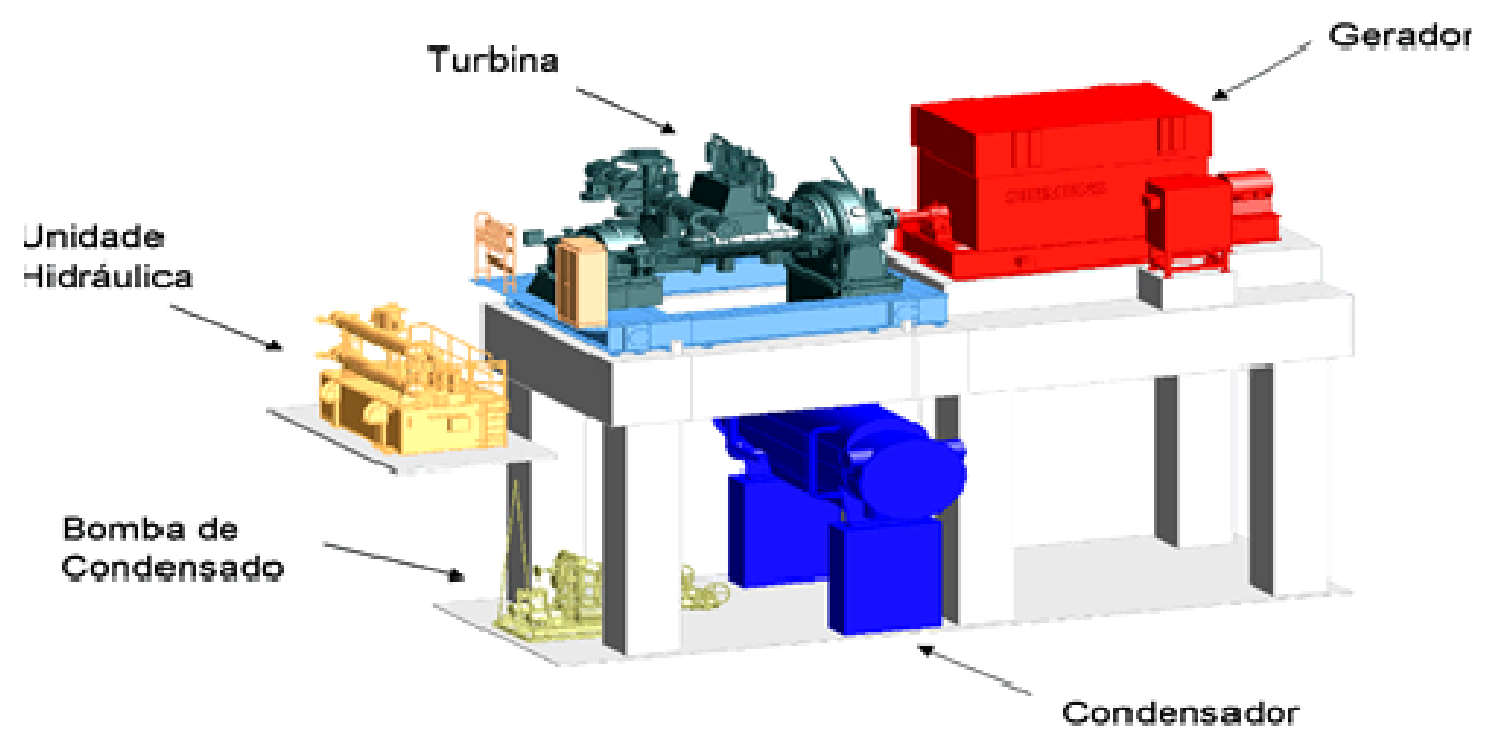

Figura 1.1 - Arranjo mecânico de turbina a vapor

A alta complexidade desse tipo de estrutura também advém do fato de que ela serve de suporte para diversos equipamentos, com diferentes tipos de funcionamento e interligados por um emaranhado de linhas de tubos. 


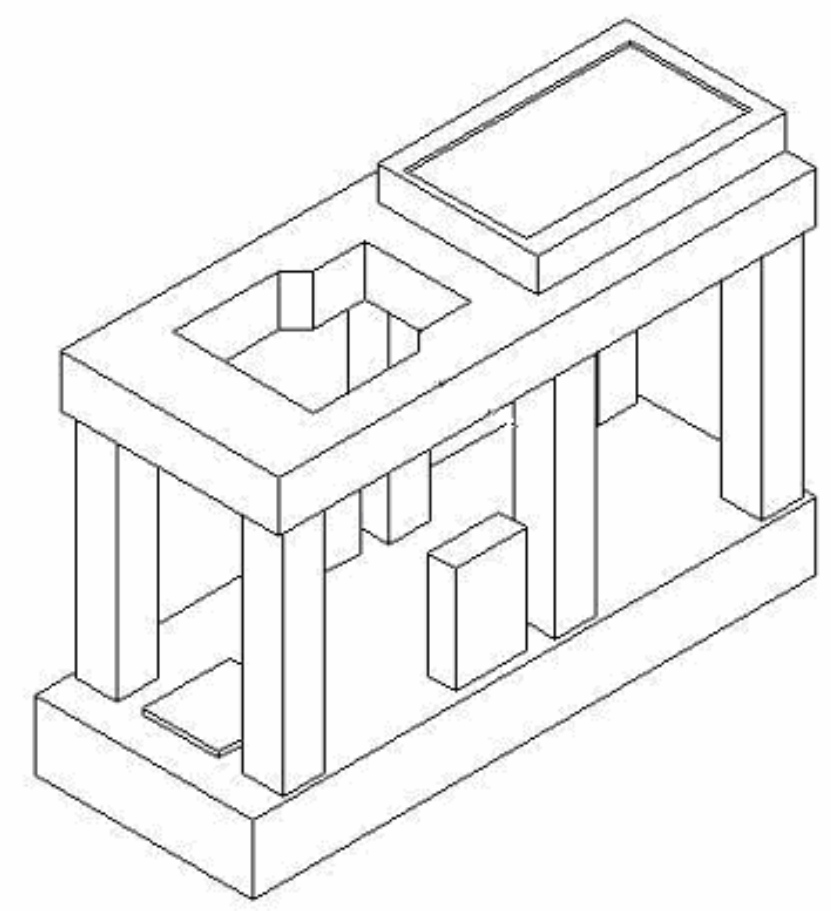

Figura 1.2 - Estrutura tipo aporticada de turbina a vapor

Por outro lado, também há estruturas de menor complexidade, como é o caso de fundações de máquinas tipo ventilador ou bomba, nas quais a base de sustentação se resume apenas a um bloco em concreto armado para um único equipamento.

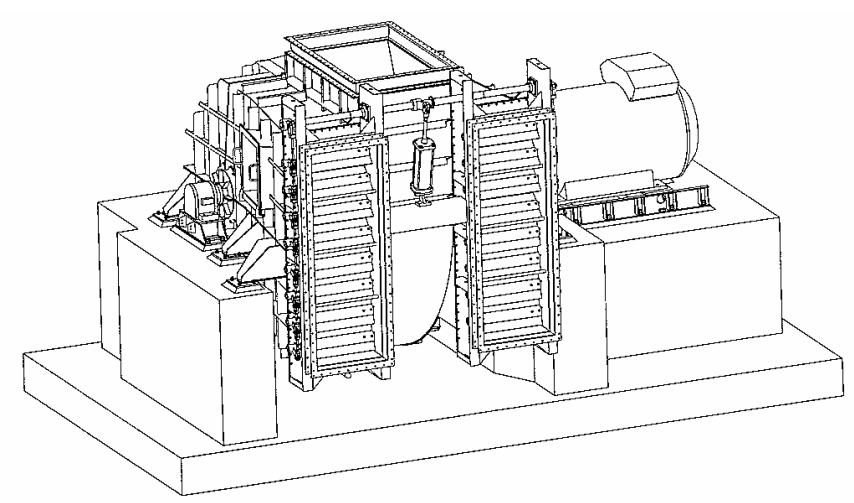

Figura 1.3 - Arranjo mecânico de ventilador centrífugo 


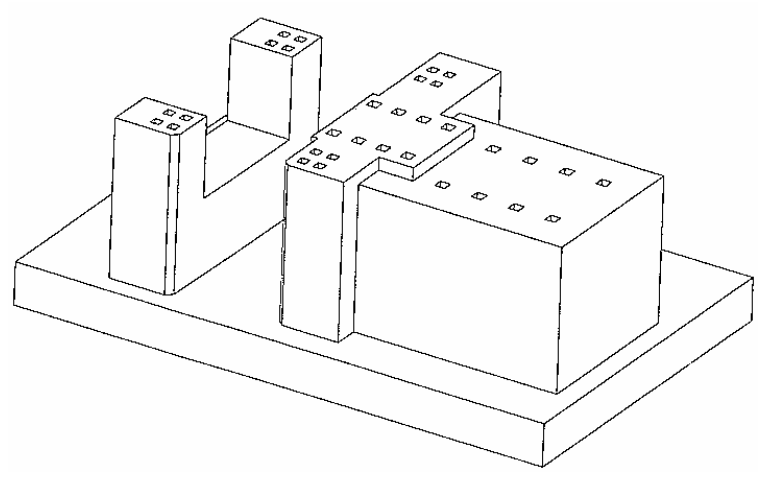

Figura 1.4 - Estrutura tipo bloco de ventilador centrífugo

Sabe-se, ainda, que muitas máquinas têm suas fundações projetadas com base em regras empíricas, as quais estabelecem, na maioria das vezes, que a massa da fundação deve ser $n$ vezes a massa da própria máquina. Essas regras não se justificam mais nos dias de hoje, com o avanço de softwares na Área de Dinâmica Estrutural.

É importante salientar que sempre que uma fundação estiver sujeita a excitações dinâmicas, a análise deve ser feita cuidadosamente de maneira a adequar as freqüências naturais da estrutura às freqüências de funcionamento do equipamento, evitando-se, assim, estados de ressonância. Uma das maneiras mais simples de afastamento das freqüências naturais da estrutura daquelas geradas pela fonte excitadora é através do enrijecimento da fundação ou aumento de sua massa.

Em adição a isso, existem métodos alternativos para evitar o estado de ressonância e redução de vibrações; são os chamados métodos de controle passivo de vibrações, os quais consistem na apropriada manipulação das características do sistema. Um dos mais comuns e eficientes métodos de redução de vibrações ressonantes em estruturas é o Amortecedor de Massa Sintonizado, usualmente conhecido pela sigla TMD (Tuned Mass Damper). Um TMD, nada mais é do que um dispositivo modular composto de uma massa $m$, uma rigidez $k$ e um amortecedor $c$, ajustados de modo a sincronizar a freqüência natural do TMD com a freqüência do modo a ser amortecido. 
A título de curiosidade, há outros métodos de controle de vibrações, que têm sido objeto de estudo de diferentes pesquisadores, como os Absorvedores de Vibrações de Coluna Líquida (LCVA), os Controladores por Impacto de Massas (ID), bem como os Amortecedores de Coluna Líquida Sintonizado (TLCD), assunto analisado em publicação de Felix et al. (2005b).

Geralmente, a análise de estruturas sujeitas a vibrações não leva em consideração a interação da estrutura com a fonte de excitação, o que simplifica a modelagem matemática do sistema e facilita a sua análise. Nesse caso, o sistema é dito ideal, porque é constituído de uma fonte de energia denominada ideal. Pode-se, então, dizer que os sistemas ideais compreendem os casos de vibrações forçadas por um termo periódico externo com uma determinada freqüência e amplitude função do tempo, em que esse termo periódico é a fonte de energia do sistema, obtida a partir de motor de potência praticamente ilimitada. Entretanto, quando ocorre interação entre a estrutura e a fonte de excitação, o modelo do sistema é dito não-ideal. Além disso, é importante salientar que fontes de energia não-ideais não podem ser representadas por uma função que dependa somente do tempo. Há necessidade de se introduzir na formulação uma equação que correlacione a fonte de energia com a equação do movimento do sistema. Logo, os sistemas não-ideais apresentam um grau de liberdade a mais em relação aos sistemas ideais correspondentes (BALTHAZAR et al., 2003 e 2004).

\subsection{Objetivos}

Com base nos conceitos relacionados a sistemas não-ideais, o presente projeto pretende estudar, ou até mesmo constatar, a existência de interação entre a estrutura em concreto armado para suporte de um ventilador axial com a sua fonte de energia, seu próprio motor, que, se desbalanceado, produz quantidade limitada de energia. Objetiva-se também constatar que, nas regiões próximas a estados de ressonância, ocorrem condições instáveis no movimento. A situação é atribuída ao fato do motor não conseguir fornecer energia suficiente para que seu regime de rotação ultrapasse os picos de ressonância do sistema, ou seja, a potência gerada pelo motor é consumida nos movimentos de grande amplitude da estrutura e não 
mais na mudança do regime de rotação. Mesmo que o motor consiga ultrapassar esse regime de rotação, não existem soluções estáveis em determinadas faixas de freqüência, o que acarreta grandes saltos. O fenômeno é conhecido como Efeito Sommerfeld (1904), também chamado de "fenômeno do salto".

Uma análise a ser feita relaciona-se às conseqüências ocasionadas aos sistemas, quando observados somente de forma ideal.

\subsection{Plano da Dissertação}

Com o intuito de antecipar o conteúdo desta dissertação, o capítulo 2 apresenta um relato histórico sobre as pesquisas pioneiras, bem como aquelas que têm sido realizadas atualmente sobre sistemas com fontes de energia não-ideais, conceitos básicos sobre fontes ideais e não-ideais de energia e descrição do fenômeno Sommerfeld.

No capítulo 3, são apresentadas noções fundamentais sobre os motores elétricos, descrição de seus principais componentes, suas curvas de conjugado e resistente da carga e os vários critérios de classificação e categorias dos motores elétricos.

No capítulo 4, deduz-se as equações do movimento para sistema ideal e não-ideal e, a partir delas, são elaboradas as rotinas de cálculo para obtenção das respostas numéricas dos sistemas, por meio do Método de Integração Runge Kutta.

O capítulo 5 aborda os materiais a serem analisados e a metodologia empregada.

O capítulo 6 traz os resultados obtidos para os sistemas analisados, segundo a metodologia apresentada no capítulo 5.

Por fim, faz-se a discussão sobre os resultados obtidos no capítulo 7 e apresentamse as conclusões no capítulo 8. 


\section{REVISÃO DA LITERATURA}

\subsection{Aspectos Históricos}

Em 1904, a partir de experiências realizadas para verificar a interação entre um motor e a estrutura que lhe dava suporte, por meio da monitoração das freqüências e das amplitudes da resposta do sistema, Sommerfeld constatou o "fenômeno do salto", ou descontinuidade na freqüência de rotação, que ficou conhecido como Efeito Sommerfeld, em sua homenagem. Tratava-se da instabilidade presente na rotação da máquina, em certas faixas de potência, nas regiões próximas da ressonância. Este foi o primeiro registro que se teve de problemas ligados a fontes não-ideais, conforme informação de V. O. Kononenko, em seu clássico livro, Vibrating Systems with Limited Power Supply (1969), inteiramente voltado ao assunto. De acordo com o mesmo autor, na citada obra, depois de 14 anos, os experimentos de Sommerfeld foram repetidos e estendidos por Timoshenko, que também não conseguiu explicar completamente o mecanismo de interação do sistema oscilante com sua fonte de energia.

A clássica obra de Kononenko aponta, em 1939, um outro estudioso do assunto, Kalischuk, que se preocupou em discutir substancialmente o mesmo fenômeno da interação existente entre um sistema oscilante e uma fonte de energia, embora a análise não se ativesse a relacionar sua dependência às características do motor.

Em 1940, ainda na esteira de Kononenko, Martyshkin publicou o primeiro trabalho que relatou uma descrição qualitativa precisa da interação de um sistema oscilante com uma determinada fonte de energia. Martyshkin avaliou que as oscilações instáveis de um sistema linear, na região da ressonância, estavam diretamente relacionadas com as características do motor elétrico.

Segundo a obra de Kononenko, em 1949, Rocard encontrou a solução para o problema de um sistema excitado pela força de inércia de uma massa girante desbalanceada. Neste trabalho, Rocard constatou a existência de regiões de 
oscilações instáveis, porém não conseguiu definir a posição exata em que ocorriam. Em 1955, Mazet retomou o assunto e conseguiu definir com exatidão essas regiões de instabilidade.

Dois anos antes, Blekhman considerou o problema da auto-sincronização de várias massas girantes desbalanceadas e conseguiu obter uma base comum para a representação de qualquer sistema oscilante com um grau de liberdade. A equação do movimento encontrada por Blekhman para o sistema, com uma massa desbalanceada, foi igual à de Rocard. Mais tarde, em 1994, esse mesmo assunto viria a ser estudado por Nóbrega, que, em Auto-Sincronização de motores nãoideais apoiados em estruturas elásticas, analisou o fenômeno da auto-sincronização de dois motores elétricos de corrente contínua. A análise apresentou resultados vinculados aos fenômenos relacionados à estabilidade dinâmica do sistema, em razão da não-linearidade da fonte de excitação, como também demonstrou os efeitos decorrentes da auto-sincronização dos rotores, que tendem a entrar em sincronia com velocidade angular e diferença de fase.

Fazendo ainda referência ao trabalho de Blekhman, este concluiu que era possível um sistema oscilante fazer um "salto" de transição de um estado ressonante para um não-ressonante, assumindo que o torque do motor era proporcional à velocidade angular. Os resultados encontrados por Blekhman foram utilizados para explicar o fenômeno observado por Sommerfeld.

Em 1958, Kononenko estudou problemas relativos à interação de um sistema oscilante linear com uma fonte de energia, introduzindo a característica estática da fonte de energia na equação do movimento. No mesmo ano, Pust estudou um problema semelhante, e ambos descobriram o mesmo caráter de interação do sistema oscilante com a fonte de energia, por meio de testes experimentais, em modelos mecânicos, que confirmaram a validade dos resultados obtidos. Então, foi natural assumir que tal interação deveria se manifestar não somente no estado estacionário, mas também no estado não estacionário do movimento, especialmente no caso de interesse de passagem do sistema oscilante pela ressonância. Essa transição foi estudada por Kononenko, também em 1958, que chegou à conclusão de que a variação da freqüência de oscilação do sistema estava intimamente ligada 
com a amplitude. Na região de grandes amplitudes, a velocidade de transição do sistema caía rapidamente na ressonância, enquanto que para pequenas amplitudes esta velocidade aumentava.

Kononenko foi a primeira pessoa a estudar, em 1959, a interação de um sistema oscilante com $n$ graus de liberdade e uma fonte de energia, chegando à conclusão de que todas características encontradas em um sistema de um grau de liberdade ocorrem em cada região de ressonância, se o movimento é estacionário ou não.

O primeiro trabalho realizado sobre a interação de um sistema oscilante sujeito a uma força restabelecedora não-linear e a sua fonte de energia foi feito em parceria, na qual Korablev estudou modelos mecânicos experimentais e discutiu o comportamento de um sistema não-linear, enquanto Kononenko e Frolov determinavam de forma mais completa o estado estacionário de oscilação e as propriedades do sistema. Este trabalho mostrou que as propriedades de ressonância de um sistema oscilante não-linear dependiam profundamente das propriedades da fonte de energia.

Em 1969, Kononenko aprofundou e ampliou o estudo até então realizado, tendo como foco a análise dos sistemas oscilatórios lineares e não-lineares expostos à excitação por fontes com potência limitada de energia. Para além de análises realizadas anteriormente, que desconsideravam 0 movimento da estrutura, Kononenko preocupou-se com a influência do movimento estrutural, apresentando formulações alternativas para a questão.

Nayfeh e Mook, em seu livro Nonlinear Oscillations (1979), discutiram o experimento realizado por Kononenko e Korablev em 1959, contribuindo com o assunto por meio da comparação de resultados experimentais e teóricos.

Novas contribuições foram dadas por Brasil e Mook (1994) ao analisarem a ação de um motor não-ideal sobre um pórtico plano e as vibrações não-lineares decorrentes. O estudo levou em consideração tanto o movimento vertical quanto o horizontal da estrutura, reproduzindo o Efeito Sommerfeld através de solução numérica, fenômeno que foi observado experimentalmente por Garzeri em 2001, em sua tese de 
doutorado. Nela, além do Efeito Sommerfeld, Garzeri verificou a ocorrência dos fenômenos de ressonância interna e saturação modal.

Ainda em 2001, Balthazar juntamente com outros estudiosos analisaram numericamente a passagem pela ressonância de um sistema vibratório, com dois graus de liberdade, excitado por uma fonte não-ideal de energia. Suas observações resultaram em uma lei de controle do sistema durante sua passagem pelo primeiro pico de ressonância.

Em 2002, Felix apresentou em sua tese de doutorado um modelo matemático destinado ao estudo analítico-numérico das vibrações não-lineares decorrentes de um problema não-ideal, tendo por base o modelo experimental estudado por Gazeri em 2001. Felix desenvolveu e analisou algoritmos de controle por saturação para sistemas não-ideais, utilizando simulação computacional.

Dando prosseguimento aos estudos sobre os sistemas vibratórios não-ideais, Balthazar et al. (2003) realizaram estudo panorâmico, selecionando e resumindo pesquisas até então publicadas sobre o assunto. O estudo mostrou que os modelos descritos encontravam-se muito próximos de situações reais de ordem prática.

Nesse mesmo ano de 2003, Kuroiwa, em sua dissertação de mestrado, analisando as vibrações ocorridas nas estruturas suportes de equipamentos rotativos desbalanceados, avaliou a possibilidade de seu controle passivo por TMD. As nãolinearidades inerentes ao comportamento não-ideal do motor foram consideradas em estudo paramétrico, que envolveu a manipulação das características do sistema.

Em 2004, Balthazar, Brasil e Garzeri publicaram On non-ideal simple portal frame structural model: experimental results under non-ideal excitation, trabalho conciso, mas importante, sobre os sistemas não-ideais. Nele os autores discutiram os fenômenos físicos envolvidos na fonte de energia, no motor e na estrutura e apresentaram uma proposta metodológica para tratá-los adequadamente.

Outra publicação de Balthazar e outros marca o ano de 2004: A review of new vibration issues due to non ideal energy sources, na qual os pesquisadores fazem 
uma ampla revisão bibliográfica sobre as fontes não-ideais, numa importante contribuição.

Tsuchida e outros (2005) voltaram-se à análise numérica de um sistema vibratório não-linear e não-ideal com dois graus de liberdade e ressonância interna 1:2, avaliando suas vibrações regulares e irregulares.

Feitosa, em sua dissertação de mestrado (2006), procurou analisar possíveis aplicações práticas no controle de vibrações em duas situações: um pórtico de suporte a motor não-ideal e uma torre de suporte a gerador eólico, ambos desbalanceados. Objetivou também demonstrar que o amortecimento por impacto pode controlar o indesejável fenômeno Sommerfeld.

\subsection{Diferença entre os Modelos de Fonte de Energia Ideal e Não-Ideal}

\subsubsection{Fonte de Energia Ideal}

Conforme conceito anteriormente apresentado, o modelo de fonte de energia ideal é aquele em que a fonte atua sobre o sistema oscilante, mas não sofre influência recíproca do sistema; isto é, independe das condições de movimento e pode ser representada por uma função explícita do tempo.

A força de excitação gerada por esse tipo de fonte, no caso de um motor elétrico desbalanceado, é dada por:

$P(t)=F \cdot \sin \left(\omega_{m} \cdot t\right)$

em que $\omega_{m}$ é a freqüência de rotação do motor, sendo:

$F=m_{d} \cdot a_{r}$ 
$a_{r}=\frac{V^{2}}{r}=\frac{\left(r \cdot \omega_{m}\right)^{2}}{r}=r \cdot \omega_{m}^{2}$

em que $r$ é uma excentricidade de balanceamento de massas.

Introduzindo as Eq. (2.2) e Eq. (2.3) na Eq. (2.1), a força de excitação do motor é obtida através da Eq. (2.4).

$P(t)=m_{d} \cdot r \cdot \omega_{m}^{2} \cdot \sin \left(\omega_{m} \cdot t\right)$

Logo, em sistemas ideais, o problema maior está em controlar ou até mesmo evitar amplificações dinâmicas que ocorrem nos estados de ressonância, fenômeno decorrente da proximidade da freqüência de operação da fonte (motor) com a da freqüência natural da estrutura.

\subsubsection{Fonte de Energia Não-Ideal}

O modelo de fonte de energia não-ideal é aquele em que a fonte atua sobre o sistema oscilante e, ao mesmo tempo, sofre uma ação recíproca do sistema, sendo que eventuais alterações nos parâmetros do sistema podem ser acompanhadas por alterações nas condições de funcionamento da fonte. Diz-se, então, que há interação entre a fonte e a estrutura que a suporta. Em determinadas situações, tal interação pode se tornar muito significativa, principalmente no caso em que a fonte possui energia limitada.

A interação entre a fonte de energia (motor) e a estrutura deve-se ao fato de que parte da energia fornecida pela fonte é consumida para movimentar a estrutura. A energia consumida pela estrutura pode ser calculada pela Eq.(2.5).

$E\left(\omega_{m}\right)=\frac{C}{2 \cdot \omega_{m}} \cdot \omega^{2} \cdot \rho^{2}$ 
em que:

$\rho \quad$ amplitude do movimento

$\omega \quad$ freqüência natural da estrutura

C coeficiente de amortecimento da estrutura

sendo:

$\rho=\frac{P_{0}}{K} \cdot D$

$P_{0}=m_{d} \cdot r \cdot \omega_{m}^{2}$

$D=\frac{1}{\sqrt{\left(1-\beta^{2}\right)^{2}+(2 \cdot \xi \cdot \beta)^{2}}}$

$\beta=\frac{\omega_{m}}{\omega}$

$C=2 \cdot \xi \cdot M \cdot \omega$

em que:

$K \quad$ rigidez da estrutura

$D$ fator de amplificação dinâmica

$\xi \quad$ taxa de amortecimento

A figura 2.1 mostra a curva da energia $E\left(\omega_{m}\right)$ consumida pela estrutura. Observando-se o gráfico, pode-se constatar que a quantidade de energia consumida para movimentar a estrutura é crescente à medida que a amplitude de vibração aumenta, atingindo valores máximos nos estados de ressonância. 


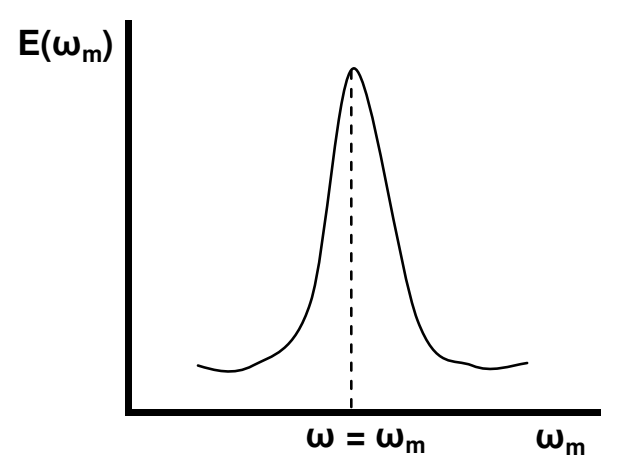

Figura 2.1 - Curva de energia consumida $E\left(\omega_{m}\right)$ pela estrutura

Como a influência de uma fonte de energia não-ideal em um sistema oscilante depende do estado de seu movimento, é impossível expressar tal ação com uma função explícita do tempo. Assim, um sistema oscilante com fonte de energia nãoideal deve ser analisado de maneira particular.

\subsection{Características das Fontes de Energia}

Geralmente, as características das fontes de energia são definidas através de curvas que retratam relações entre grandezas. A escolha adequada da grandeza a ser considerada depende do tipo de energia. Grandezas convenientes para energia elétrica normalmente são tensão e corrente elétrica; já para a energia mecânica, podem ser torque e velocidade angular, ou força e velocidade linear.

Assume-se que tais características representam as propriedades essenciais da fonte de energia, as quais determinam seu estado permanente de funcionamento e, por essa razão, são freqüentemente nomeadas como estáticas.

As características das fontes de energia mecânica, que ocorrem em sistemas mecânicos, normalmente são especificadas por relações entre conjugados $L$ e $H$ versus velocidade de rotação $\omega_{m}$, como se pode observar nas figuras 2.2 e 2.3. 


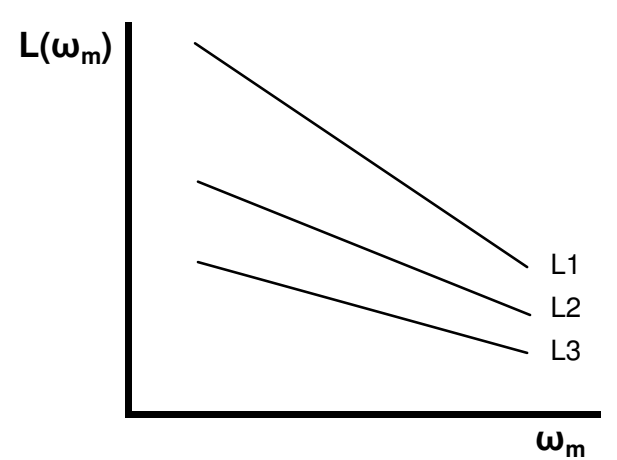

Figura 2.2 - Curvas características $L\left(\omega_{m}\right)$ da fonte

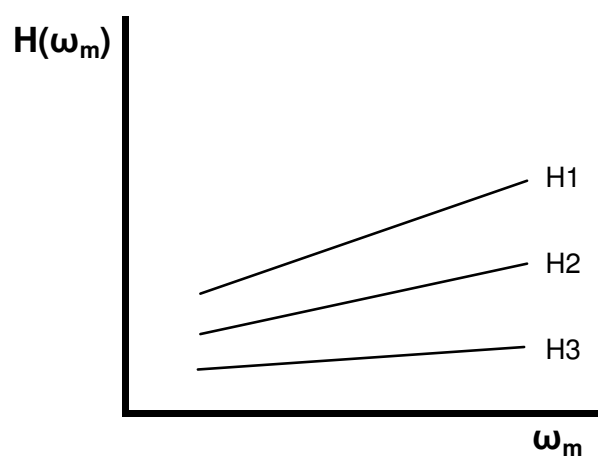

Figura 2.3 - Curvas dos conjugados resistentes internos $H\left(\omega_{m}\right)$ da fonte

Cada par de curva $L$ e $H$ apresentado ( $L 1$ e $H 1, L 2$ e $H 2, L 3$ e $H 3$ ) relaciona-se a uma posição de um dispositivo para regulagem da fonte de energia. É importante salientar que tanto a função $L\left(\omega_{m}\right)$ como a $H\left(\omega_{m}\right)$ são determinadas de forma experimental e assumidas como conhecidas neste trabalho.

\subsection{Estabilidade de Sistema com Fonte de Energia Não-Ideal}

Segundo estudos realizados por Kononenko (1969), em sistemas mecânicos, o estado permanente de funcionamento de um sistema não-ideal, ou seja, a situação em que a rotação da fonte de energia permanece constante, ocorre no ponto de interseção da curva característica $L\left(\omega_{m}\right)$ referente a uma determinada potência fornecida pela fonte, com a curva de energia total consumida pelo sistema, dada pela Eq. (2.11).

$S\left(\omega_{m}\right)=E\left(\omega_{m}\right)+H\left(\omega_{m}\right)$ 
A figura 2.4 retrata a situação de estabilidade de sistema não-ideal, em que a rotação da fonte (motor) é definida quando o sistema atinge um estado estacionário, caracterizado pela equivalência entre as energias fornecidas ao sistema e aquelas consumidas, tanto para movimentar a estrutura $E\left(\omega_{m}\right)$, quanto para atuar nas resistências internas do motor $H\left(\omega_{m}\right)$.

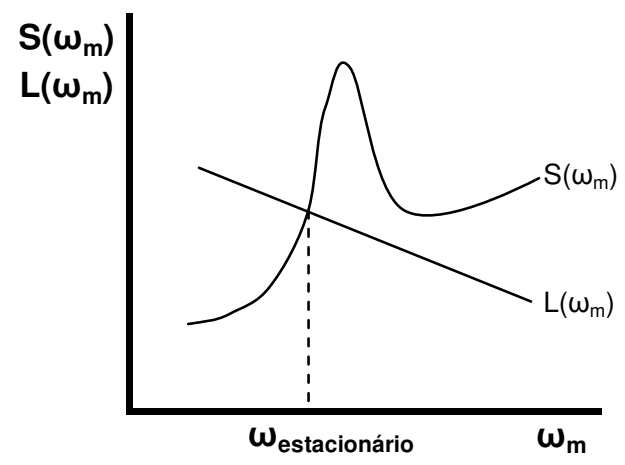

Figura 2.4 - Ponto de estabilidade de sistema não-ideal

Portanto, em sistema não-ideal observa-se que a resposta do sistema influencia a fonte de energia, que, por sua vez, é responsável pelo movimento da estrutura; ou seja, há uma interação entre a fonte e a estrutura, fazendo com que a análise do sistema se torne bem mais complexa quando comparada a sistemas ideais.

\subsection{Fenômenos Relacionados à Fonte Não-Ideal}

Nayfeh e Mook (1979) discutiram o experimento realizado por Kononenko e Korablev (1959), reproduzindo o Efeito Sommerfeld.

O modelo físico utilizado por Kononenko e Korablev era constituído por uma viga (2) de rigidez $K$, com uma das extremidades engastada (3) e a outra fixada a um motor elétrico (1), com o rotor desbalanceado por um furo (4). A figura 2.5 mostra o modelo do experimento. 


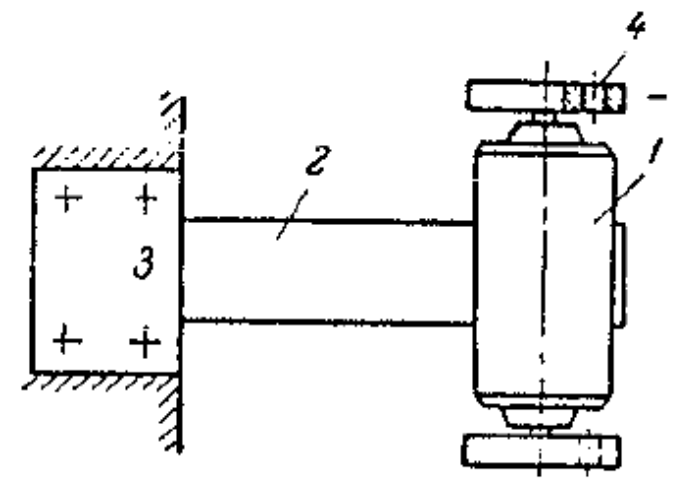

Figura 2.5 - Modelo físico experimental de Kononenko e Korablev

O experimento tinha por objetivo analisar as respostas de um motor não-ideal submetido à variação controlada de energia fornecida ao sistema. Dessa maneira, Kononenko e Korablev obtiveram o par de valores "amplitude x freqüência de rotação do motor" para cada nível de conjugado, no momento em que o sistema alcançava o regime permanente.

No entanto, sabendo-se que o regime permanente do sistema é definido na interseção da curva $S\left(\omega_{m}\right)$ com a curva característica $L\left(\omega_{m}\right)$ do motor, conforme a figura 2.4, e tendo em vista que um motor pode apresentar diferentes curvas características (por exemplo: L1 a L5), quando submetido a diferentes níveis de energia, têm-se, então, diferentes pontos de funcionamento para o sistema, conforme a figura 2.6 .

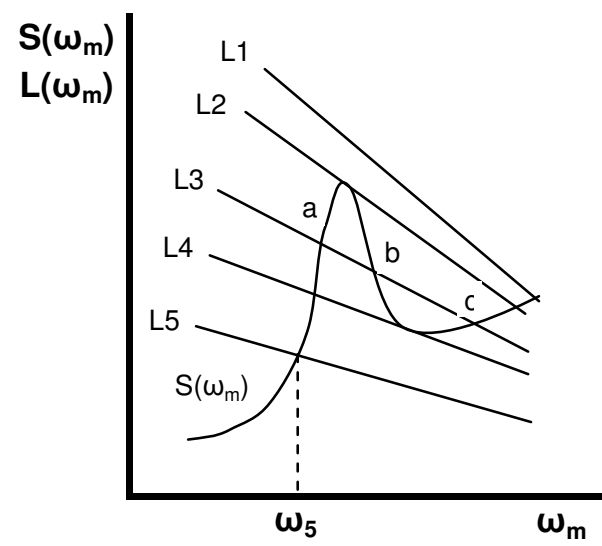

Figura 2.6 - Curvas características $L\left(\omega_{m}\right)$ do motor e curva $S\left(\omega_{m}\right)$ 
Fornecendo-se potência crescente ao sistema, tem-se inicialmente a interseção das curvas $L 5$ e $S\left(\omega_{m}\right)$, definindo o estado permanente de funcionamento do sistema com rotação $\omega_{5}$. Aplicando-se uma potência maior ao sistema, obtém-se um novo ponto de estabilidade, quando a curva $L 4$ intercepta $S\left(\omega_{m}\right)$. Esse foi o procedimento adotado por Kononenko e Korablev para obter os vários pares de valores "amplitude x freqüência de rotação do motor", para potência elétrica crescente e decrescente, plotados na figura 2.7 .

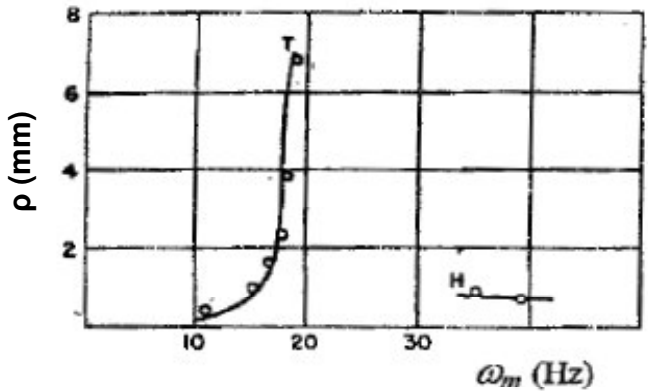

(a) $\omega_{m}$ crescente

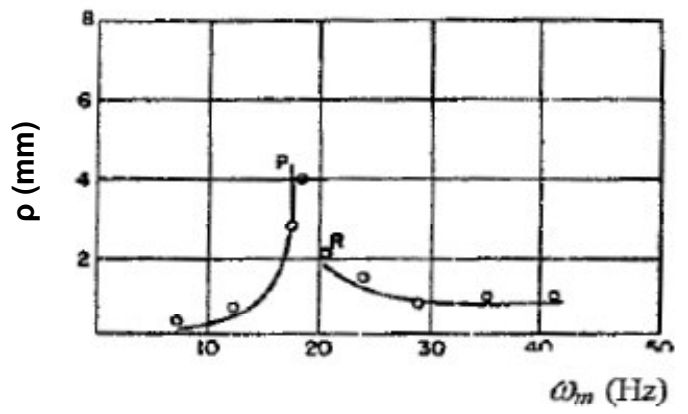

(b) $\omega_{\mathrm{m}}$ decrescente

Figura 2.7 - Curvas teóricas e experimentais obtidas por Kononenko e Korablev

Observando-se a figura 2.6, constata-se que a curva $L 3$ intercepta $S\left(\omega_{m}\right)$ em três pontos, a saber: $a, b$ e $c$. No entanto, o ponto $a$ é o que define o ponto de estabilidade do sistema, uma vez que está sendo analisado o controle crescente na potência elétrica fornecida ao sistema. Sabe-se, ainda, que o ponto $b$ é instável e o ponto $c$ estável; isso porque, a estabilidade do sistema só é verificada quando as curvas que se interceptam apresentam derivadas de sinais contrários, ou seja, uma em sentido crescente e a outra em sentido decrescente. As descontinuidades das curvas, observadas na figura 2.7, indicam os intervalos de instabilidade do sistema e caracterizam o fenômeno conhecido como Efeito Sommerfeld (1904) ou "fenômeno do salto". O ponto $T$ da figura 2.7 refere-se ao primeiro ponto de interseção das curvas $L 2$ e $S\left(\omega_{m}\right)$ da figura 2.6, assim como o ponto $H$ refere-se ao segundo ponto de interseção das mesmas curvas. Analisando-se, agora, o controle decrescente da potência elétrica fornecida ao sistema, observa-se que o intervalo de instabilidade é menor, sendo definido pelos pontos $P$ e $R$ da figura 2.7, que se referem aos pontos de interseção das curvas $L 4$ e $S\left(\omega_{m}\right)$ da figura 2.6. 
A figura 2.8 correlaciona as Figuras 2.7 (a) e 2.7 (b), mostrando as diferentes respostas do sistema, quando submetido à fonte de energia ideal e não ideal.

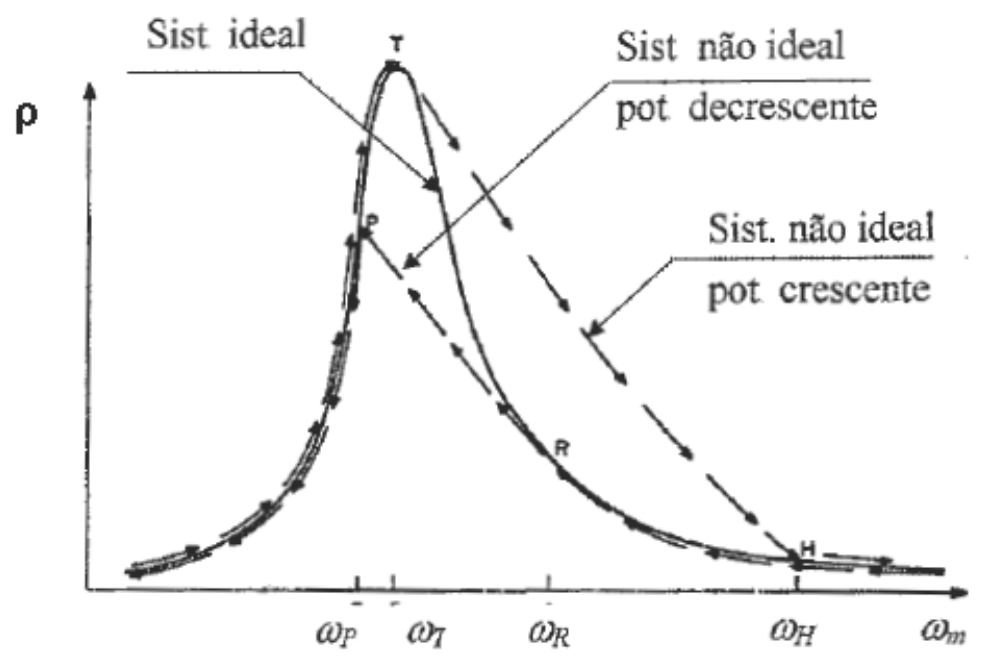

Figura 2.8 - Comparação entre sistemas ideal e não-ideal

Analisando-se $\circ$ gráfico apresentado na figura 2.8, vê-se que a extremidade esquerda da curva representa a faixa em que a potência elétrica fornecida pela fonte é relativamente baixa, mas, à medida que a potência elétrica aumenta, a amplitude do sistema cresce consideravelmente. Entretanto, a freqüência de rotação do motor não se altera de modo significativo, podendo tal fato ser observado principalmente no trecho compreendido entre os pontos $P$ e $T$ sobre a curva. Já, quando o sistema está operando na altura do ponto $T$, um pequeno acréscimo na potência elétrica causa modificação repentina no movimento, ocasionando uma expressiva diminuição na amplitude de vibração do sistema e um aumento significativo em sua freqüência. É isso que caracteriza o "fenômeno do salto".

Assim, o experimento realizado por Kononenko e Korablev pôde constatar que, nas regiões próximas ao estado de ressonância, em certas faixas de potência, ocorrem condições instáveis no movimento, acarretando grandes saltos. Isso pode ser visto na figura 2.8, onde, para potência crescente, o salto ocorre do ponto $T$ ao $H$, enquanto que, para potência decrescente, o salto ocorre do $R$ ao $P$. 


\section{NOÇÕES FUNDAMENTAIS SOBRE MOTORES}

\subsection{Motor Elétrico}

O setor industrial é responsável pela demanda de aproximadamente $45 \%$ de toda a energia elétrica consumida no país, e dentro desse setor o consumo de motores elétricos é estimado em cerca de $75 \%$, fato que evidencia a grande importância do conhecimento deste tipo de equipamento por parte dos engenheiros e técnicos.

Neste estudo, ênfase maior foi dada aos motores trifásicos, também conhecidos como motores assíncronos ou motores de indução, pois representam cerca de $90 \%$ da potência de motores fabricados e são os mais difundidos nas aplicações de engenharia, por sua simplicidade de utilização, versatilidade e custo.

A finalidade básica dos motores é o acionamento de máquinas e equipamentos mecânicos, cabendo ao usuário a correta seleção do motor para cada processo industrial. $\mathrm{O}$ processo de seleção dos motores deve satisfazer basicamente três requisitos:

- condições ambientais: temperatura, agressividade do meio, altitude etc;

- especificações sobre a alimentação: tensão, freqüência, tipo da fonte etc;

- características, exigências da carga e condições de serviço, tais como rotação, conjugados, confiabilidade exigida pelo processo industrial, ciclo de operação, esforços mecânicos, potência solicitada etc.

Sabe-se, ainda, que existem três tipos de motores elétricos: os de corrente contínua, os de corrente alternada e os universais. 


\subsubsection{Motores Elétricos de Corrente Contínua}

Esses motores apresentam custo mais elevado e, além disso, necessitam de uma fonte de corrente contínua, ou de um dispositivo que converta a corrente alternada em contínua. Podem operar com velocidade ajustável entre amplos limites e se prestam a controles de grande flexibilidade e precisão. Por essa razão, seu uso é restrito a casos especiais em que estas exigências compensem o custo mais elevado da instalação. Dentre o universo dos motores de corrente contínua, os mais utilizados são os de excitação paralela e série.

\subsubsection{Motores Elétricos de Corrente Alternada}

São os mais utilizados, uma vez que a distribuição de energia elétrica é feita geralmente em corrente alternada. Os principais tipos são:

a) motor síncrono: funciona com velocidade fixa e é usado somente para grandes potências, ou quando há necessidade de que a velocidade seja invariável;

b) motor de indução, normalmente opera com uma velocidade constante, a qual varia ligeiramente com a carga mecânica aplicada ao eixo. Atualmente é o motor mais empregado no acionamento de máquinas, por ser simples, versátil e de baixo custo. Uma característica interessante é que, hoje em dia, pode-se controlar a sua velocidade com o auxilio de inversores de freqüência. 
A figura 3.1 mostra o universo tecnológico de motores elétricos

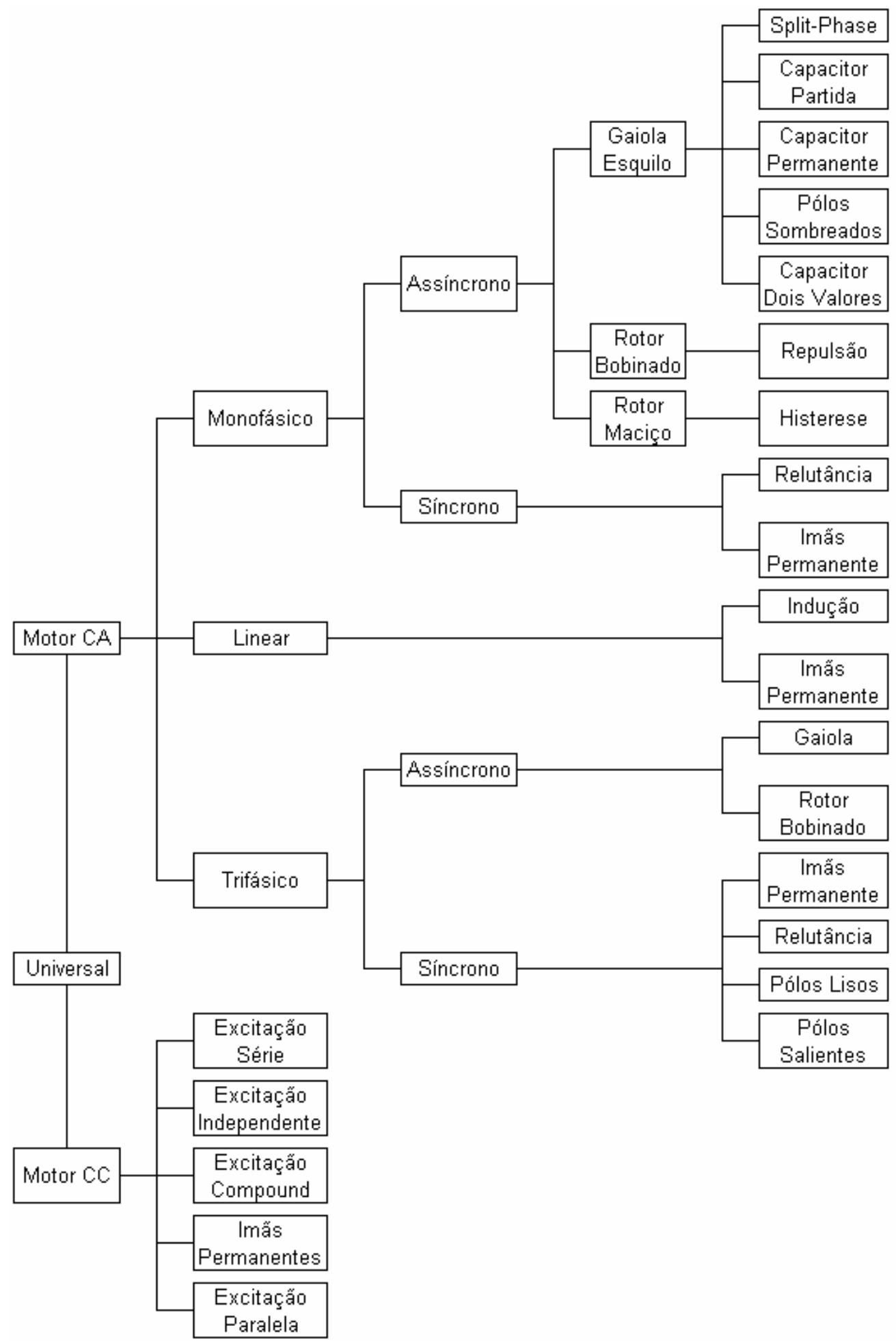

Figura 3.1 - Universo tecnológico de motores elétricos 
As figuras 3.2 e 3.3 mostram os principais componentes de um motor elétrico de indução trifásico.

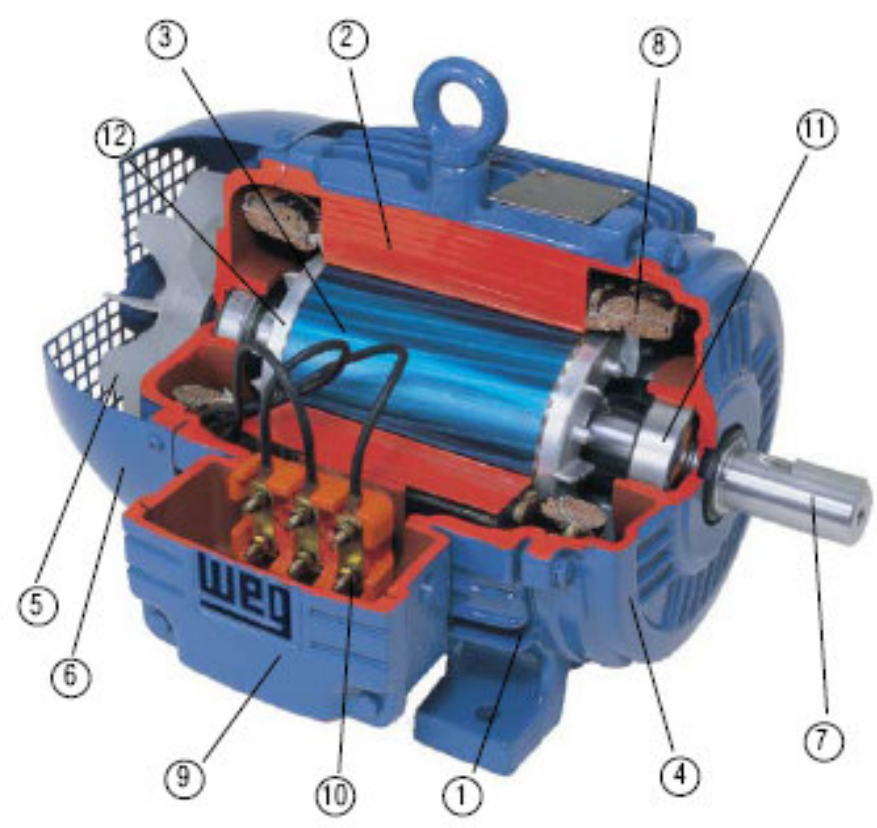

Figura 3.2 - Motor elétrico de indução trifásico

O motor de indução trifásico é composto basicamente de duas partes: estator e rotor.

- Estator

1 - carcaça: estrutura suporte do conjunto; de construção robusta em ferro fundido, aço ou alumínio injetado, resistente à corrosão e com aletas;

2 - núcleo de chapas: chapas em aço magnético, tratadas termicamente para reduzir ao mínimo as perdas no ferro;

8 - enrolamento trifásico: três conjuntos iguais de bobinas, uma para cada fase, formando um sistema trifásico que é ligado à rede trifásica de alimentação. 
- Rotor

7 - eixo: transmite a potência mecânica desenvolvida pelo motor; é tratado termicamente para evitar problemas como empenamento e fadiga;

3 - núcleo de chapas: essas chapas possuem as mesmas características das chapas do estator;

12 - barras e anéis de curto-circuito: são constituídas de alumínio injetado sob pressão, formando numa única peça.

- Outras partes do motor de indução trifásico

4 - tampa;

5 - ventilador;

6 - tampa defletora;

9 - caixa de ligação;

10 - terminais;

11 - rolamentos.

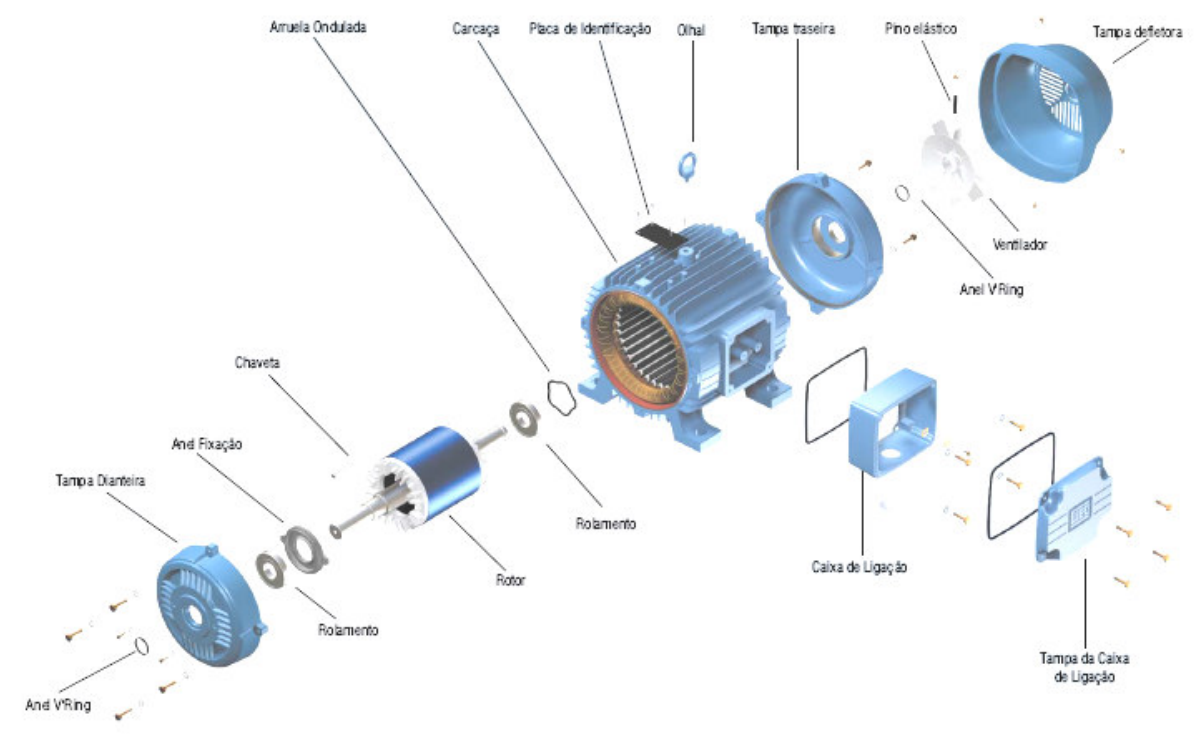

Figura 3.3 - Partes integrantes de motor elétrico 


\subsection{Curvas dos Conjugados Motor e Resistente da Carga}

Define-se a medida do esforço necessário para girar um eixo como sendo o conjugado do motor, também chamado torque, momento ou binário. De acordo com a natureza da carga mecânica, haverá uma curva de conjugado resistente associada a ela. Em cargas de ventilação, o conjugado resistente é proporcional ao quadrado da velocidade, enquanto que em guindastes e pontes rolantes, o conjugado resistente é praticamente constante. A figura 3.4, apresenta a curva do conjugado acelerante para um motor de indução.

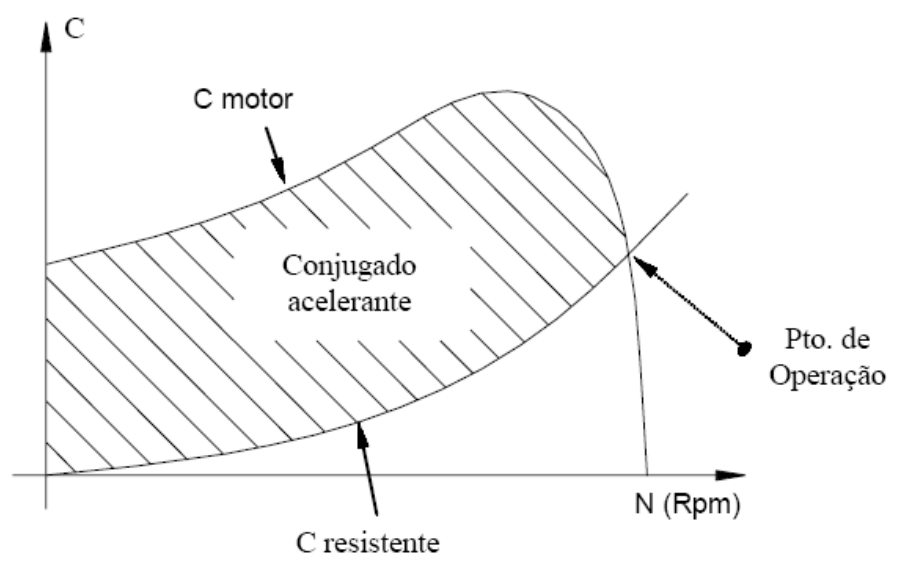

Figura 3.4 - Conjugados do motor e resistente da carga

A figura 3.5 mostra as curvas de conjugado em função da rotação para diferentes tipos de motores.

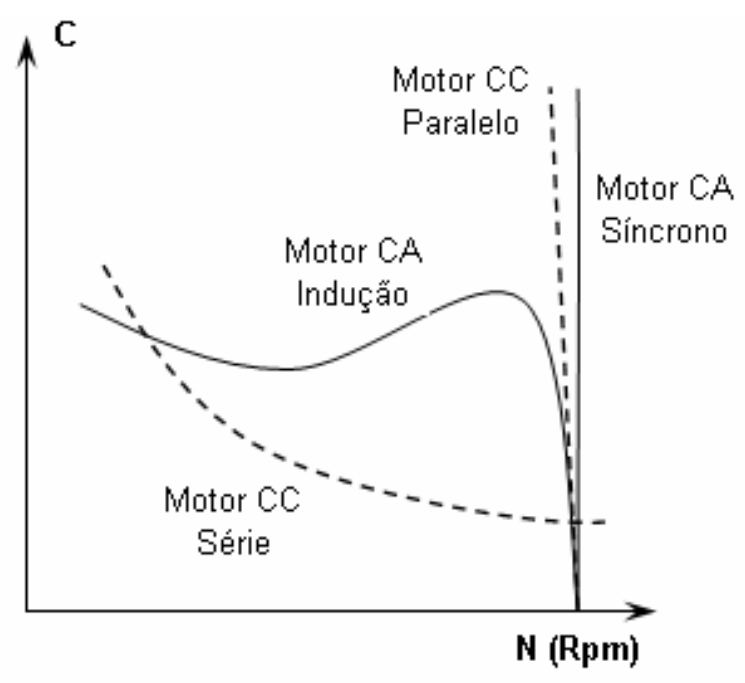

Figura 3.5 - Curvas de conjugado x Velocidade dos diferentes tipos de motor 


\subsection{Categorias de Conjugados}

De acordo com as características de conjugado em relação à velocidade e corrente de partida, os motores de indução trifásicos são classificados em categorias, cada uma adequada a um tipo de carga. As diferentes categorias são observadas na figura 3.6.

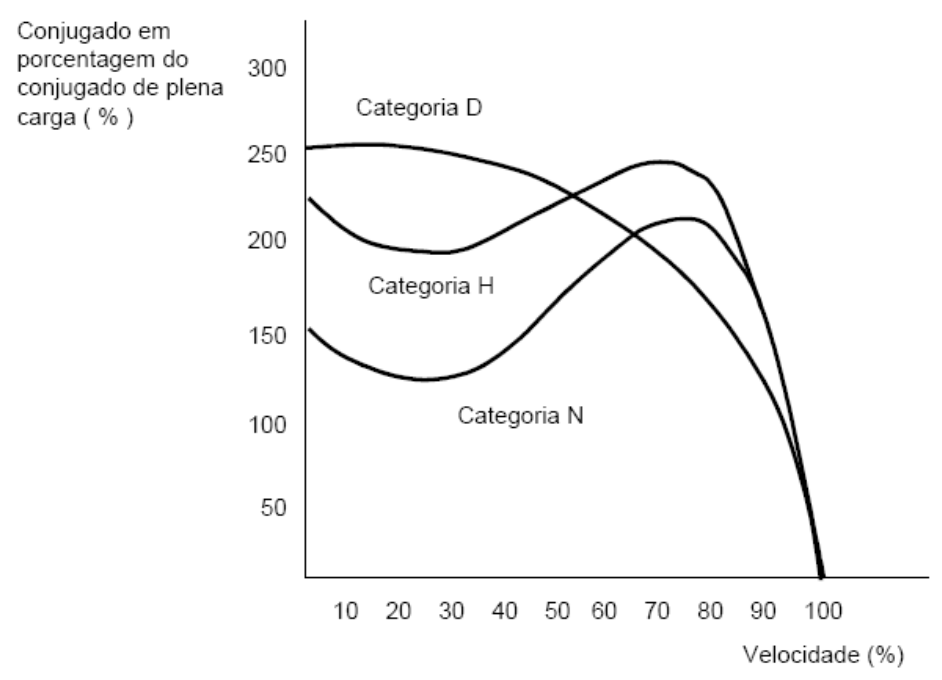

Figura 3.6 - Curvas de conjugado x Velocidade das diferentes categorias

Tais conjugados têm as seguintes aplicações:

- categoria N: conjugado e corrente de partida normal, baixo escorregamento. Abrange a maior parte dos motores encontrados no mercado e se destinam a cargas normais, tais como bombas, máquinas operatrizes e ventiladores;

- categoria $\mathrm{H}$ : alto conjugado de partida, corrente de partida normal, baixo escorregamento. Recomendado para esteiras transportadoras, peneiras, trituradores e britadores;

- categoria D: alto conjugado de partida, corrente de partida normal, alto escorregamento. Usados em prensas excêntricas, elevadores e acionadores de cargas com picos periódicos. 


\subsection{Curvas Características Conjugado / Veloc. das Cargas Mecânicas}

No universo das cargas mecânicas a serem acionadas, podem-se destacar tipos básicos que obedecem à equação geral abaixo:

$C_{r}=C_{0}+\left(C_{n}-C_{0}\right) \cdot\left(\frac{\omega}{\omega_{n}}\right)^{n}$

sendo:

$C_{r} \quad$ conjugado resistente

$C_{0} \quad$ conjugado resistente para $\omega=0$

$C_{n} \quad$ conjugado resistente nominal

$\omega$ velocidade

$\omega_{n} \quad$ velocidade nominal

\subsubsection{Cargas de Conjugado Resistente Constante $(n=0)$}

Ocorrem quando o seu conjugado se mantém inalterado para qualquer valor da velocidade do acionamento, resultando em $C_{r}=C_{n}$. Fazem parte desses tipos de carga: as esteiras transportadoras, pontes rolantes, cadeira do laminador de chapa e máquinas de atrito seco.

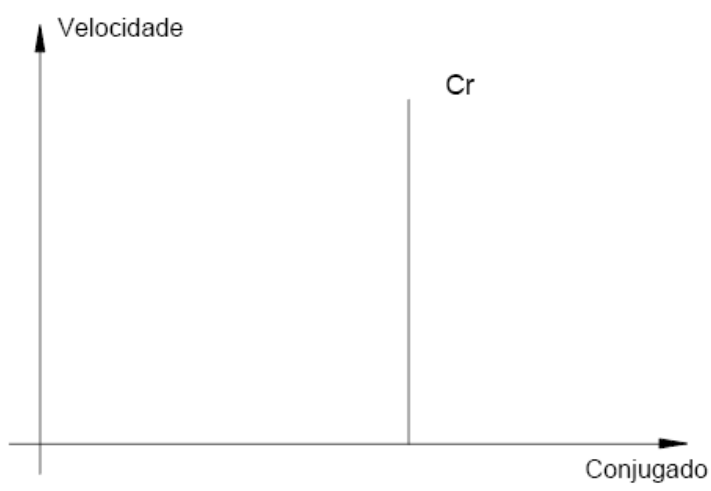

Figura 3.7 - Velocidade x Conjugado para $n=0$ 
3.4.2 Cargas de Conjugado Resistente Linear com a Velocidade $(n=1)$

Ocorrem quando o conjugado varia linearmente em função da velocidade, resultando em:

$C_{r}=C_{0}+\left(C_{n}-C_{0}\right) \cdot\left(\frac{\omega}{\omega_{n}}\right)$

Fazem parte desses tipos de carga: os sistemas de acoplamento hidráulico ou eletromagnético, geradores com alto fator de potência e transmissão de torque por atrito viscoso.

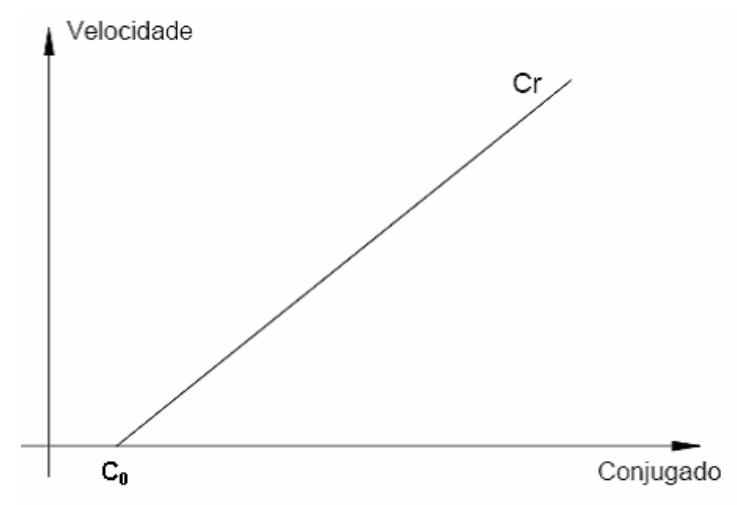

Figura 3.8 - Velocidade $\times$ Conjugado para $n=1$

3.4.3 Cargas de Conjugado Resistente Cresc. com Quadrado da Vel. $(n=2)$

Ocorrem quando o conjugado varia em relação à velocidade segundo uma parábola, resultando em:

$C_{r}=C_{0}+\left(C_{n}-C_{0}\right) \cdot\left(\frac{\omega}{\omega_{n}}\right)^{2}$

Fazem parte desses tipos de carga: as bombas centrífugas e os ventiladores. 


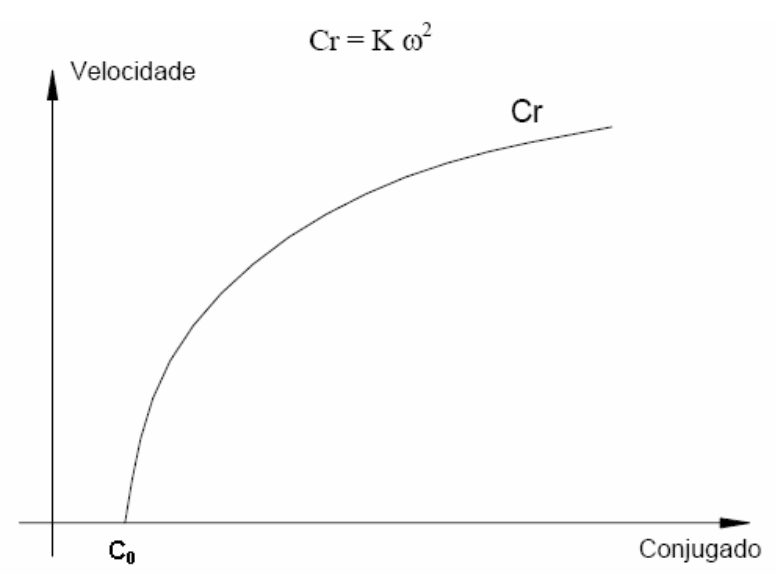

Figura 3.9 - Velocidade x Conjugado para $n=2$

\subsection{Velocidade Síncrona $\left(n_{s}\right)$}

A velocidade síncrona do motor é definida pela velocidade de rotação do campo girante. O valor dessa velocidade depende da maneira como estão distribuídas e ligadas as bobinas no estator do motor, bem como da freqüência da corrente que circula pelo enrolamento estatórico.

Prova-se que esta velocidade vale:

$n_{s}=\frac{60 \cdot f_{r}}{p}$

Para as freqüências e "polaridades" usuais, as velocidades síncronas são apresentadas na tabela 3.1 .

Tabela 3.1 - Velocidades síncronas

\begin{tabular}{ccc}
\hline \multirow{2}{*}{ No de Pólos $^{2}$} & \multicolumn{2}{c}{ Rotação Sincrona (rpm) } \\
& 60 Hertz & 50 Hertz \\
\hline 2 & 3600 & 3000 \\
4 & 1800 & 1500 \\
6 & 1200 & 1000 \\
8 & 900 & 750 \\
10 & 720 & 600 \\
\hline
\end{tabular}




\section{FORMULAÇÃO: SISTEMA COM FONTE IDEAL E NÃO-IDEAL DE ENERGIA}

\subsection{Sistema com Fonte Ideal de Energia}

$\mathrm{Na}$ situação ideal o sistema em estudo possui uma única coordenada generalizada $Q_{1}=v$, conforme indicado na figura 4.1, a qual também mostra as outras principais características do sistema.

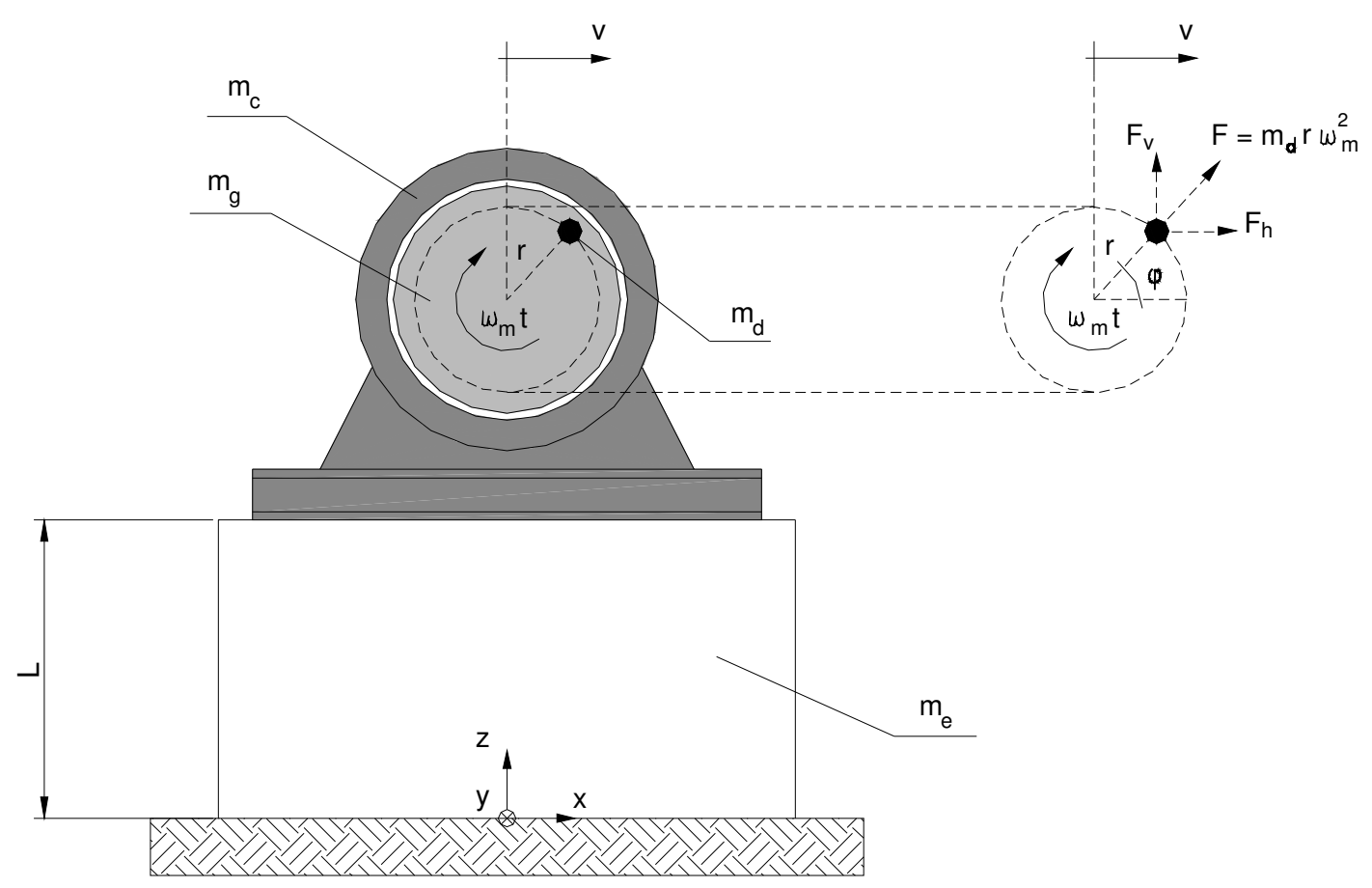

Figura 4.1 - Modelo para formulação: sistema ideal e não-ideal

\subsubsection{Cálculo das Características Dinâmicas Generalizadas da Estrutura}

O sistema apresentado acima constitui um modelo físico contínuo. Para determinação das características do modelo, tais como massa e rigidez dinâmicas generalizadas, torna-se necessário discretizá-lo, de modo que ele possa ser representado por equações diferenciais ordinárias que governem seu movimento. 
A solução clássica para o problema da determinação de soluções para as equações diferenciais parciais representativas de um sistema físico contínuo baseia-se na substituição de uma certa função $u$ (escalar ou vetorial), a solução exata, desconhecida, por aproximações construídas pela superposição de funções do tipo:

$u \approx \hat{u}=\Phi_{0}+\sum_{i=1}^{n} \Phi_{i} \cdot \mathrm{B}_{i}$

em que $\mathrm{B}_{i}(i=1,2,3, \ldots, n)$ são parâmetros a serem computados para um "bom ajuste". Funções desse tipo são chamadas "trial functions" e suas componentes $\Phi$, "shape functions", ou funções de forma.

\subsubsection{Cálculo da Massa Generalizada da Estrutura $m_{e}$}

O cálculo da massa generalizada da estrutura $m_{e}$ é feito por meio da Eq. (4.1), sendo que $\Phi(z)$, definida pela a Eq. (4.2), representa uma função de forma. Neste caso, $\Phi(z)$, por apresentar uma ótima aproximação para o modelo em estudo, é a única componente da "trial function".

$m_{e}=m_{l} \cdot \int_{0}^{l} \Phi^{2}(z) \cdot d z$

$\Phi(z)=1-\cos \left(\frac{\pi}{2} \cdot \frac{z}{l}\right)$

$\Phi^{2}(z)=1-2 \cdot \cos \left(\frac{\pi}{2} \cdot \frac{z}{l}\right)+\cos ^{2}\left(\frac{\pi}{2} \cdot \frac{z}{l}\right)$

Introduzindo a Eq. (4.3) na Eq. (4.1), obtém-se:

$$
m_{e}=\left(\frac{3}{2}-\frac{4}{\pi}\right) \cdot m_{l} \cdot l
$$

que permite calcular o valor de $m_{e}$. 


\subsubsection{Cálculo da Rigidez Generalizada da Estrutura $K$}

Da mesma maneira que a massa $m_{e}$ foi calculada, pode-se calcular a rigidez generalizada da estrutura $K$, através da Eq. (4.5), sendo $K_{g}$ a rigidez geométrica e $K_{f}$ a rigidez a flexão.

$K=K_{f}-K_{g}$

$K_{g}=P \cdot \int_{0}^{l} \Phi^{\prime 2}(z) \cdot d z$

$K_{f}=E \cdot I \cdot \int_{0}^{l} \Phi^{\prime 2}(z) \cdot d z$

sendo:

$\Phi^{\prime}(z)=\frac{\pi}{2 \cdot l} \cdot \sin \left(\frac{\pi}{2} \cdot \frac{z}{l}\right)$

$\Phi^{\prime \prime}(z)=\frac{\pi^{2}}{4 \cdot l^{2}} \cdot \cos \left(\frac{\pi}{2} \cdot \frac{z}{l}\right)$

$\Phi^{\prime 2}(z)=\frac{\pi^{2}}{4 \cdot l^{2}} \cdot \sin ^{2}\left(\frac{\pi}{2} \cdot \frac{z}{l}\right)$

$\Phi^{\prime 2}(z)=\frac{\pi^{4}}{16 \cdot l^{4}} \cdot \cos ^{2}\left(\frac{\pi}{2} \cdot \frac{z}{l}\right)$ 
Introduzindo as Eq. (4.10) e (4.11) nas Eq. (4.6) e (4.7), respectivamente, e em seguida, substituíndo-as na Eq. (4.5), obtém-se:

$$
K=\frac{\pi^{4} \cdot E \cdot I}{32 \cdot l^{3}}-\frac{\pi^{2} \cdot P}{8 \cdot l}
$$

que permite calcular o valor de $K$. Nota-se que a primeira parcela corresponde à chamada rigidez elástica e a segunda à chamada rigidez geométrica, que leva em conta o estado de carregamento axial da estrutura.

\subsubsection{Cálculo da Energia Cinética do Sistema}

No caso ideal, a energia cinética total do sistema é determinada pela Eq. (4.13).

$$
T=\frac{1}{2} \cdot\left(m_{c}+m_{g}\right) \cdot \dot{v}^{2}+\frac{1}{2} \cdot m_{l} \cdot \int_{0}^{l} \Phi^{2}(z) \cdot d z \cdot \dot{v}^{2}+\frac{1}{2} \cdot J_{m} \cdot \omega_{m}^{2}
$$

sendo:

$$
m_{m}=m_{c}+m_{g}
$$

Introduzindo a Eq. (4.1) e Eq. (4.14) na Eq. (4.13), obtém-se:

$$
T=\frac{1}{2} \cdot\left[m_{m} \cdot \dot{v}^{2}+m_{e} \cdot \dot{v}^{2}+J_{m} \cdot \omega_{m}^{2}\right]
$$

que permite calcular o valor de $T$. 


\subsubsection{Cálculo da Energia Potencial do Sistema}

Para o sistema ideal, o cálculo da energia potencial $V$ é feito utilizando-se a Eq. (4.16), sendo $U$ a energia potencial de deformação, calculada pela Eq. (4.17), e $W^{c}$ o trabalho das forças conservativas.

$V=U-W^{c}$

$U=\frac{1}{2} \cdot\left[E \cdot I \cdot \int_{0}^{l} \Phi^{\prime 2}(z) \cdot d z-P \cdot \int_{0}^{l} \Phi^{\prime 2}(z) \cdot d z\right] \cdot v^{2}$

A única força conservativa aplicada é a força gravitacional, que não realiza trabalho por ser perpendicular à direção do movimento. Logo $W^{c}=0$ e $V=U$.

Introduzindo as Eq. (4.6) e (4.7) na Eq. (4.17), obtém-se:

$U=\frac{1}{2} \cdot\left(K_{f}-K_{g}\right) \cdot v^{2}=\frac{1}{2} \cdot K \cdot v^{2}$

e conseqüentemente:

$V=\frac{1}{2} \cdot K \cdot v^{2}$

\subsubsection{Cálculo da Força não Conservativa}

Para sistema ideal, a força $F$ de desbalanceamento ocasionada por um motor elétrico pode ser calculada pela Eq. (4.20).

$$
F=m_{d} \cdot a_{r}
$$


sendo:

$a_{r}=\frac{V^{2}}{r}=\frac{\left(r \cdot \omega_{m}\right)^{2}}{r}=r \cdot \omega_{m}^{2}$

Introduzindo a Eq. (4.21) na Eq. (4.20), obtém-se:

$F=m_{d} \cdot r \cdot \omega_{m}{ }^{2}$

Logo, as forças de desbalanceamento ocasionadas pela massa desbalanceada $m_{d}$ e excentricidade $r$, na direção vertical e horizontal, são obtidas pelas Eq. (4.23) e (4.24), respectivamente.

$$
\begin{aligned}
& F_{v}=F \cdot \sin \left(\omega_{m} \cdot t\right)=m_{d} \cdot r \cdot \omega_{m}{ }^{2} \cdot \sin \left(\omega_{m} \cdot t\right) \\
& F_{h}=F \cdot \cos \left(\omega_{m} \cdot t\right)=m_{d} \cdot r \cdot \omega_{m}{ }^{2} \cdot \cos \left(\omega_{m} \cdot t\right)
\end{aligned}
$$

Agora, utilizando o teorema do trabalho virtual, em que $F_{v}$ não realiza trabalho por ser perpendicular à direção do movimento, a força não conservativa é obtida pela Eq. (4.25).

$N^{n c}=-C \cdot \dot{v}+m_{d} \cdot r \cdot \omega_{m}{ }^{2} \cdot \cos \left(\omega_{m} \cdot t\right)$

admitindo-se amortecimento estrutural na forma:

$C=2 \cdot M \cdot \omega \cdot \xi$

$\omega=\sqrt{\frac{K}{M}}$

$M=m_{m}+m_{e}$

Em geral, em estruturas de concreto, a taxa de amortecimento $\xi$ pode ser assumida em torno de $2 \%$ a $5 \%$. 


\subsubsection{Equação do Movimento}

A equação do movimento é obtida empregando-se a Equação Generalizada de Lagrange:

$\frac{\partial}{\partial t}\left(\frac{\partial T}{\partial \dot{v}}\right)-\frac{\partial T}{\partial v}=-\frac{\partial V}{\partial v}+N^{n c}$

sendo:

$\frac{\partial T}{\partial \dot{v}}=\left[m_{m} \cdot \dot{v}+m_{e} \cdot \dot{v}\right]=\left[m_{m}+m_{e}\right] \cdot \dot{v}=M \cdot \dot{v}$

$\frac{\partial}{\partial t}\left(\frac{\partial T}{\partial \dot{v}}\right)=\left[m_{m} \cdot \ddot{v}+m_{e} \cdot \ddot{v}\right]=\left[m_{m}+m_{e}\right] \cdot \ddot{v}=M \cdot \ddot{v}$

$\frac{\partial T}{\partial v}=0$

$\frac{\partial V}{\partial v}=K \cdot v$

Introduzindo as Eq. (4.25), (4.31), (4.32) e (4.33) na Eq. (4.29), obtém-se a equação do movimento para o sistema ideal:

$M \cdot \ddot{v}+C \cdot \dot{v}+K \cdot v=m_{d} \cdot r \cdot \omega_{m}{ }^{2} \cdot \cos \left(\omega_{m} \cdot t\right)$

Isolando a aceleração $\ddot{v}$, obtém-se:

$\ddot{v}=\frac{m_{d} \cdot r \cdot \omega_{m}{ }^{2} \cdot \cos \left(\omega_{m} \cdot t\right)-C \cdot \dot{v}-K \cdot v}{M}$

que permite calcular o valor de $\ddot{v}$. 


\subsection{Sistema com Fonte Não-Ideal de Energia}

Na situação não-ideal, acrescenta-se mais um grau de liberdade $Q_{2}=\varphi$, referente à posição da massa desbalanceada $m_{d}$ com excentricidade $r$. A figura 4.1 também retrata a situação não-ideal para o sistema em estudo.

\subsubsection{Cálculo da Energia Cinética do Sistema}

Neste caso, inclui-se a energia cinética da massa desbalanceada $m_{d}$, conforme se verifica na Eq. (4.36).

$T=\frac{1}{2} \cdot\left(m_{c}+m_{g}\right) \cdot \dot{v}^{2}+\frac{1}{2} \cdot m_{l} \cdot \int_{0}^{l} \Phi^{2}(z) \cdot d z \cdot \dot{v}^{2}+\frac{1}{2} \cdot m_{d} \cdot v^{* 2}+\frac{1}{2} \cdot J_{m} \cdot \dot{\varphi}^{2}$

Em que a posição da massa desbalanceada $m_{d}$ é obtida pelas Eq. (4.37) e (4.38).

$x=v+r \cdot \cos \varphi$

$z=r \cdot \sin \varphi$

A partir das Eq. (4.37) e (4.38), a velocidade da massa desbalanceada $m_{d}$ é obtida por:

$\dot{x}=\dot{v}-\dot{\varphi} \cdot r \cdot \sin \varphi$

$\dot{z}=\dot{\varphi} \cdot r \cdot \cos \varphi$

sabendo-se que:

$v^{* 2}=\dot{x}^{2}+\dot{z}^{2}$ 
e introduzindo as Eq. (4.39) e (4.40) na Eq. (4.41), obtém-se:

$v^{*^{2}}=\dot{v}^{2}-2 \cdot r \cdot \dot{v} \cdot \dot{\varphi} \cdot \sin \varphi+\dot{\varphi}^{2} \cdot r^{2}$

Agora, introduzindo as Eq. (4.1) e (4.42) na Eq. (4.36), obtém-se:

$T=\frac{1}{2} \cdot\left[\left(m_{c}+m_{g}+m_{e}+m_{d}\right) \cdot \dot{v}^{2}+\left(m_{d} \cdot r^{2}+J_{m}\right) \cdot \dot{\varphi}^{2}-2 \cdot m_{d} \cdot r \cdot \dot{v} \cdot \dot{\varphi} \cdot \sin \varphi\right]$

fazendo:

$M_{0}=M+m_{d}=m_{m}+m_{e}+m_{d}=m_{c}+m_{g}+m_{e}+m_{d}$

$J_{0}=m_{d} \cdot r^{2}+J_{m}$

$S_{0}=m_{d} \cdot r$

A equação da energia cinética se resume a Eq. (4.47).

$T=\frac{1}{2} \cdot\left[M_{0} \cdot \dot{v}^{2}+J_{0} \cdot \dot{\varphi}^{2}-2 \cdot S_{0} \cdot \dot{v} \cdot \dot{\varphi} \cdot \sin \varphi\right]$

que permite calcular o valor de $T$.

\subsubsection{Cálculo da Energia Potencial do Sistema}

Novamente, o cálculo da energia potencial $V$ é realizado por meio da Eq. (4.16), sendo $U$ a energia potencial de deformação dada pela Eq. (4.18), que neste caso coincide com a do sistema ideal. 
No sistema não-ideal, a parcela do trabalho das forças conservativas $W^{c}$ é levada em consideração, em conseqüência da energia potencial gravitacional da massa desbalanceada $m_{d}$, que pode ser calculada pela Eq.(4.48).

$W^{c}=-g \cdot m_{d} \cdot r \cdot \sin \varphi=-g \cdot S_{0} \cdot \sin \varphi$

Introduzindo as Eq. (4.18) e (4.48) na Eq. (4.16), obtém-se:

$V=\frac{1}{2} \cdot K \cdot v^{2}+g \cdot m_{d} \cdot r \cdot \sin \varphi=\frac{1}{2} \cdot K \cdot v^{2}+g \cdot S_{0} \cdot \sin \varphi$

que permite calcular o valor de $V$.

\subsubsection{Cálculo da Força não Conservativa}

Para a coordenada generalizada do sistema $Q_{1}=v$, a força não conservativa é dada pela Eq. (4.50), o amortecimento estrutural:

$$
N_{v}{ }^{n c}=-C \cdot \dot{v}
$$

Para a coordenada generalizada do sistema $Q_{2}=\varphi$, o trabalho das forças não conservativas é calculado pela diferença entre o conjugado característico do motor $L_{(\dot{\varphi})}$ e o conjugado resistente interno do motor $H_{(\dot{\varphi})}$, conforme a Eq. (4.51).

$$
N_{\varphi}^{n c}=L_{(\dot{\varphi})}-H_{(\dot{\varphi})}
$$

A empresa WEG já fornece graficamente o conjugado característico líquido do motor, ou seja, $L_{(\dot{\varphi})}-H_{(\dot{\varphi})}$, apresentado no Anexo A. 
Sabe-se, ainda, que o conjugado resistente $H_{(\dot{\varphi})}$ depende exclusivamente das características internas do motor e geralmente pode ser definido pela Eq. (4.52).

$H_{(\dot{\varphi})}=K_{r} \cdot \dot{\varphi}$

sendo:

$K_{r}$ : constante relacionada com o conjugado absorvido pelas perdas mecânicas e pela resistência aerodinâmica do rotor do motor.

4.2.4 Equação do Movimento

A equação do movimento é obtida utilizando-se a formulação de Lagrange.

- Para a coordenada generalizada $Q_{1}=v$, tem-se:

$\frac{\partial}{\partial t}\left(\frac{\partial T}{\partial \dot{v}}\right)-\frac{\partial T}{\partial v}=-\frac{\partial V}{\partial v}+N_{v}{ }^{n c}$

sendo:

$\frac{\partial T}{\partial \dot{v}}=\left[M_{0} \cdot \dot{v}-S_{0} \cdot \dot{\varphi} \cdot \sin \varphi\right]$

$\frac{\partial}{\partial t}\left(\frac{\partial T}{\partial \dot{v}}\right)=\left[M_{0} \cdot \ddot{v}-S_{0} \cdot\left(\ddot{\varphi} \cdot \sin \varphi+\dot{\varphi}^{2} \cdot \cos \varphi\right)\right]$

$\frac{\partial T}{\partial v}=0$

$\frac{\partial V}{\partial v}=K \cdot v$ 
Introduzindo as Eq. (4.50), (4.55), (4.56) e (4.57) na Eq. (4.53), obtém-se a Eq. (4.58) do movimento para o sistema não-ideal referente à coordenada generalizada $Q_{1}=v$ :

$M_{0} \cdot \ddot{v}+C \cdot \dot{v}+K \cdot v=S_{0} \cdot\left(\ddot{\varphi} \cdot \sin \varphi+\dot{\varphi}^{2} \cdot \cos \varphi\right)$

- Para a coordenada generalizada $Q_{2}=\varphi$, tem-se:

$\frac{\partial}{\partial t}\left(\frac{\partial T}{\partial \dot{\varphi}}\right)-\frac{\partial T}{\partial \varphi}=-\frac{\partial V}{\partial \varphi}+N_{\varphi}{ }^{n c}$

sendo:

$\frac{\partial T}{\partial \dot{\varphi}}=\left[J_{0} \cdot \dot{\varphi}-S_{0} \cdot \dot{v} \cdot \sin \varphi\right]$

$\frac{\partial}{\partial t}\left(\frac{\partial T}{\partial \dot{\varphi}}\right)=\left[J_{0} \cdot \ddot{\varphi}-S_{0} \cdot(\ddot{v} \cdot \sin \varphi+\dot{v} \cdot \dot{\varphi} \cdot \cos \varphi)\right]$

$\frac{\partial T}{\partial \varphi}=\left[-S_{0} \cdot \dot{v} \cdot \dot{\varphi} \cdot \cos \varphi\right]$

$\frac{\partial V}{\partial \varphi}=\left[g \cdot S_{0} \cdot \cos \varphi\right]$

Introduzindo as Eq. (4.51), (4.61), (4.62) e (4.63) na Eq. (4.59), obtém-se a Eq. (4.64) do movimento para o sistema não-ideal referente à coordenada generalizada $Q_{2}=\varphi$ :

$J_{0} \cdot \ddot{\varphi}-S_{0} \cdot \sin \varphi \cdot \ddot{v}=L_{(\ddot{\varphi})}-H_{(\ddot{\varphi})}-g \cdot S_{0} \cdot \cos \varphi$ 
A partir das Eq. (4.58) e (4.64), pode-se montar o seguinte sistema:

$\left[\begin{array}{cc}-S_{0} \cdot \sin \varphi & J_{0} \\ M_{0} & -S_{0} \cdot \sin \varphi\end{array}\right] \cdot\left[\begin{array}{l}\ddot{v} \\ \ddot{\varphi}\end{array}\right]=\left[\begin{array}{l}L_{(\dot{\varphi})}-H_{(\dot{\varphi})}-g \cdot S_{0} \cdot \cos \varphi \\ \dot{\varphi}^{2} \cdot S_{0} \cdot \cos \varphi-C \cdot \dot{v}-K \cdot v\end{array}\right]$

Utilizando-se a Regra de Cramer no sistema acima, obtém-se $\ddot{v}$ através da Eq. (4.67) e $\ddot{\varphi}$ pela Eq. (4.69):

$\ddot{v}=\frac{\left|\begin{array}{cc}L_{(\dot{\varphi})}-H_{(\dot{\varphi})}-g \cdot S_{0} \cdot \cos \varphi & J_{0} \\ \dot{\varphi}^{2} \cdot S_{0} \cdot \cos \varphi-C \cdot \dot{v}-K \cdot v & -S_{0} \cdot \sin \varphi\end{array}\right|}{\left|\begin{array}{cc}-S_{0} \cdot \sin \varphi & J_{0} \\ M_{0} & -S_{0} \cdot \sin \varphi\end{array}\right|}$

$\ddot{v}=\frac{\left\lfloor-\left(L_{(\dot{\varphi})}-H_{(\dot{\varphi})}-g \cdot S_{0} \cdot \cos \varphi\right) \cdot S_{0} \cdot \sin \varphi-J_{0} \cdot\left(\dot{\varphi}^{2} \cdot S_{0} \cdot \cos \varphi-C \cdot \dot{v}-K \cdot v\right)\right]}{S_{0}{ }^{2} \cdot \sin ^{2} \varphi-J_{0} \cdot M_{0}}$

$\ddot{\varphi}=\frac{\left|\begin{array}{cc}-S_{0} \cdot \sin \varphi & L_{(\dot{\varphi})}-H_{(\dot{\varphi})}-g \cdot S_{0} \cdot \cos \varphi \\ M_{0} & \dot{\varphi}^{2} \cdot S_{0} \cdot \cos \varphi-C \cdot \dot{v}-K \cdot v\end{array}\right|}{\left|\begin{array}{cc}-S_{0} \cdot \sin \varphi & J_{0} \\ M_{0} & -S_{0} \cdot \sin \varphi\end{array}\right|}$

$\ddot{\varphi}=\frac{\left\lfloor-\left(\dot{\varphi}^{2} \cdot S_{0} \cdot \cos \varphi-C \cdot \dot{v}-K \cdot v\right) \cdot S_{0} \cdot \sin \varphi-M_{0} \cdot\left(L_{(\dot{\varphi})}-H_{(\dot{\varphi})}-g \cdot S_{0} \cdot \cos \varphi\right)\right\rfloor}{S_{0}{ }^{2} \cdot \sin ^{2} \varphi-J_{0} \cdot M_{0}}$

Para a resolução das equações diferenciais do movimento é aplicado o método de integração Runge-Kutta, com passo fixo, disponível na biblioteca interna do Programa Mathcad. 


\section{MATERIAIS E METODOLOGIA}

A investigação realizada neste trabalho, sobre a aplicabilidade da teoria de sistemas não-ideais, foi feita tomando-se um sistema composto por uma estrutura em concreto armado que serve de fundação e um motor elétrico de um ventilador industrial, conforme arranjo mostrado na figura 5.1.

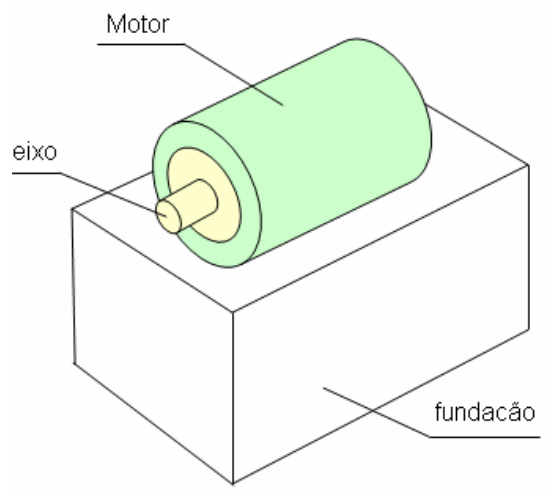

Figura 5.1 - Arranjo simplificado do sistema motor-estrutura

O sistema foi analisado sob diferentes pontos de vista; num primeiro momento, foram definidas as dimensões iniciais da base (largura de $0.75 \mathrm{~m}$, comprimento de $0.80 \mathrm{~m}$ e altura de $2.00 \mathrm{~m}$ ), decorrentes das características geométricas e mecânicas do motor, apresentadas no Anexo A.

Depois de definidas as dimensões da base, a mesma foi submetida à análise segundo a teoria de sistemas não-ideais e seus resultados foram comparados com os resultados da teoria de sistemas ideais. Os Apêndices $B$ e $C$ fornecem as rotinas de cálculo para sistema ideal e não-ideal, respectivamente.

Num segundo instante, essa mesma base foi submetida a variações de altura (de $1.50 \mathrm{~m}$ a $6.00 \mathrm{~m}$ ) e continuou sendo analisada segundo a teoria de sistemas nãoideais; porque tal situação pode ser encontrada em outros tipos de estrutura, como é o caso de um tipo de torre de resfriamento de água, figuras 5.2 e 5.3, em que a concepção estrutural retrata a mesma situação em estudo, ou seja, uma base de concreto como apoio para um motor elétrico. 


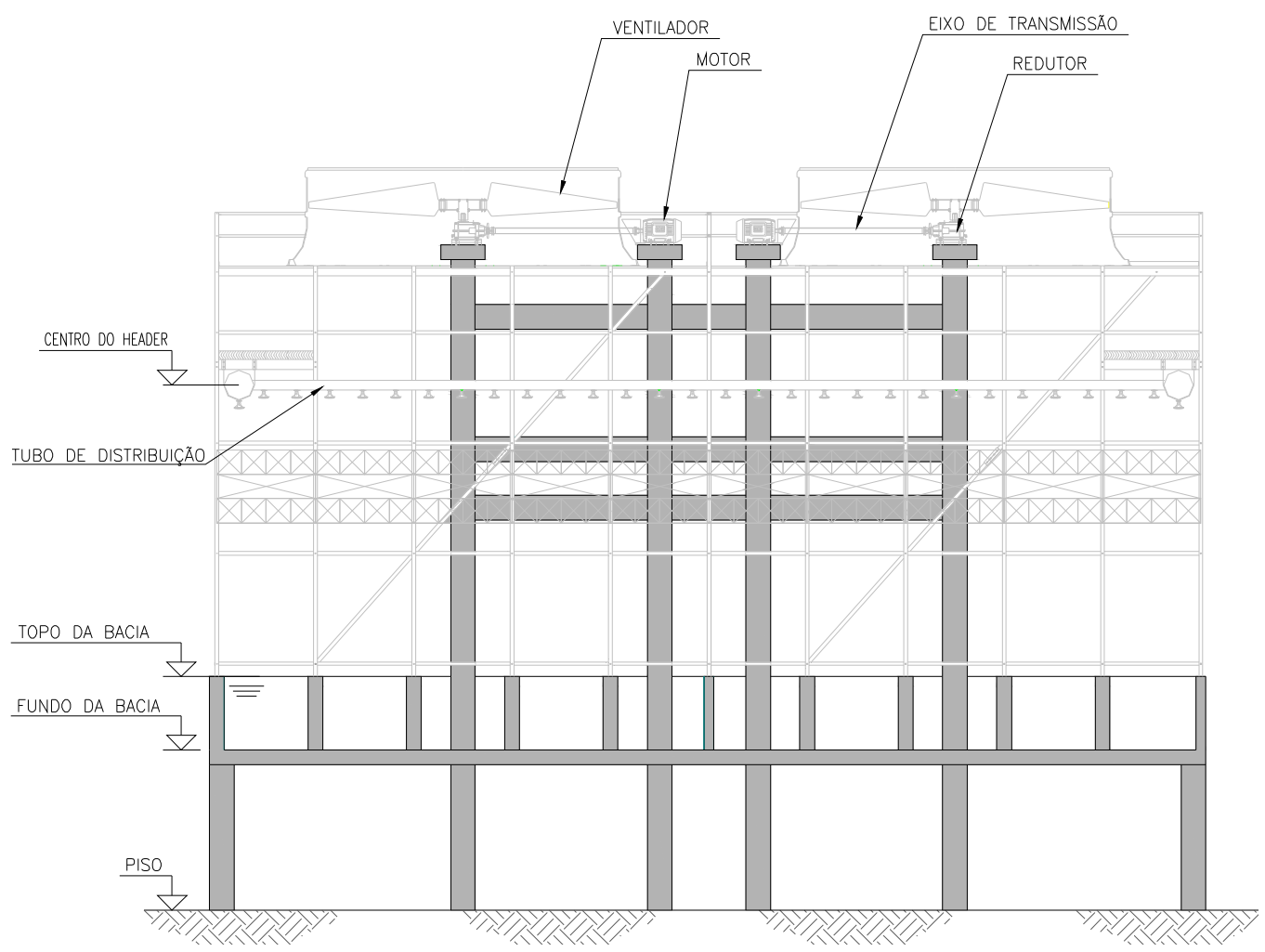

Figura 5.2 - Seção longitudinal de uma torre de resfriamento de água

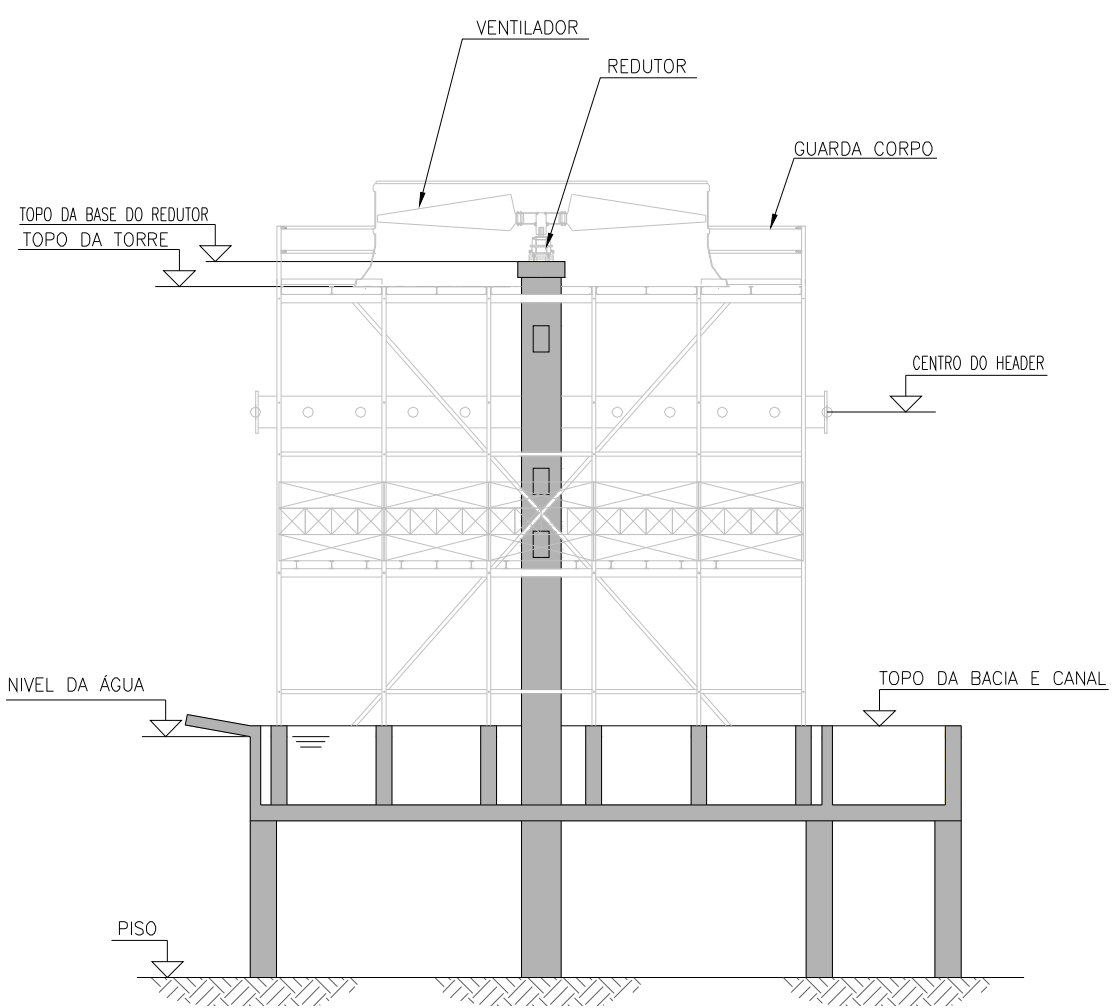

Figura 5.3 - Seção transversal de uma torre de resfriamento de água 
Finalmente, a base de concreto foi acrescida de $0.20 \mathrm{~m}$; primeiro, na direção de maior inércia e, em seguida, na direção de menor inércia. Em ambas as situações houve variação da altura. Os resultados foram analisados e comparados com aqueles gerados a partir da análise das dimensões atribuídas à base inicial.

Importante salientar que a análise segundo a teoria de sistemas ideais e não-ideais foi feita seguindo as recomendações da Norma ISO 1940/1, que define qualidade de balanceamento como sendo o produto da excentricidade da massa girante $(65 \%$ da massa total do motor, conforme informação do fabricante WEG Equipamentos Elétricos S/A - Motores) pela freqüência de rotação do motor, no caso $\omega_{m}=187.45 \mathrm{rad} / \mathrm{s}$. Para ventiladores esse valor é $r \cdot \omega_{m}=6,3 \mathrm{~mm} / \mathrm{s}$.

Com o intuito de aprofundar mais o estudo, além de seguir as recomendações da Norma ISO 1940/1 no que diz respeito à qualidade de balanceamento para ventiladores, os sistemas foram submetidos à análise com outros valores de qualidade de balanceamento não recomendados pela Norma, a saber: $r \cdot \omega_{m}=2000 \mathrm{~mm} / \mathrm{s}$ e $r \cdot \omega_{m}=4000 \mathrm{~mm} / \mathrm{s}$. O Apêndice $\mathrm{D}$ apresenta os gráficos das respostas dos sistemas em estudo.

A formulação, empregada na análise, segundo a teoria de sistemas ideais e nãoideais de energia, foi desenvolvida para um modelo de poucos graus de liberdade, em que as equações do movimento foram deduzidas a partir da formulação de Lagrange. Para a resolução das equações diferenciais do movimento, foi aplicado o método de integração Runge-Kutta, com passo fixo, disponível na biblioteca interna do Programa Mathcad, da empresa MathSoft.

As informações inerentes do motor, como curvas de conjugado característico e resistente interno, foram fornecidas pela WEG. Notar que a curva característica apresentada no Anexo A já é a curva característica líquida do motor $L_{(\dot{\varphi})}-H_{(\dot{\varphi})}$, ou seja, estão descontadas as perdas referentes às resistências internas do motor; isso porque o fabricante gera essa curva com o motor em funcionamento. Portanto, a curva de energia total consumida pelo sistema $S\left(\omega_{m}\right)$, refere-se somente à parcela de energia consumida pela estrutura $E\left(\omega_{m}\right)$ para se movimentar. 
Como o gráfico da curva característica líquida do motor encontra-se admensionalizado (conjugado em relação ao conjugado nominal $\mathrm{C} / \mathrm{C}$ n $\mathrm{x}$ rotação em relação à rotação síncrona em \%), é necessário e fundamental colocá-lo num sistema comum de unidade de trabalho ( $\mathrm{Nm} \times \mathrm{rad} / \mathrm{s})$. O Apêndice A apresenta os valores correspondentes a essa equivalência de unidade e também fornece um procedimento, através de regressão polinomial para o aplicativo Mathcad, para ajustar o grau do menor polinômio que melhor se aproxima dos valores de entrada; no caso, o polinômio correspondente é o de $12^{\circ} \mathrm{grau}$. 


\section{RESULTADOS}

6.1 Sistema Ideal e Não-Ideal com $r \cdot \omega_{m}=6,3 \mathrm{~mm} / \mathrm{s}$

Os resultados apresentados abaixo referem-se à análise, segundo a teoria de sistemas ideais e não-ideais de energia, da base de concreto de dimensões iniciais iguais a $0.75 \mathrm{~m}$ de largura, $0.80 \mathrm{~m}$ de comprimento e $2.00 \mathrm{~m}$ de altura.

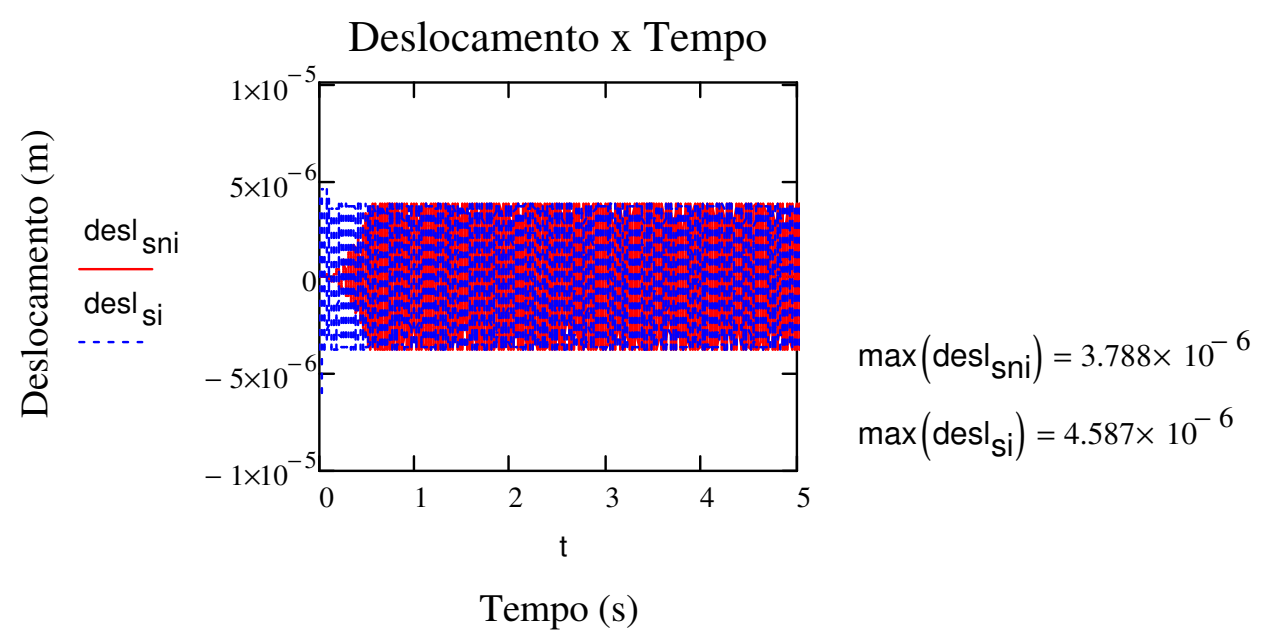

Gráfico 6.1 - Deslocamentos: sistema ideal e não-ideal com $r \cdot \omega_{m}=6,3 \mathrm{~mm} / \mathrm{s}$

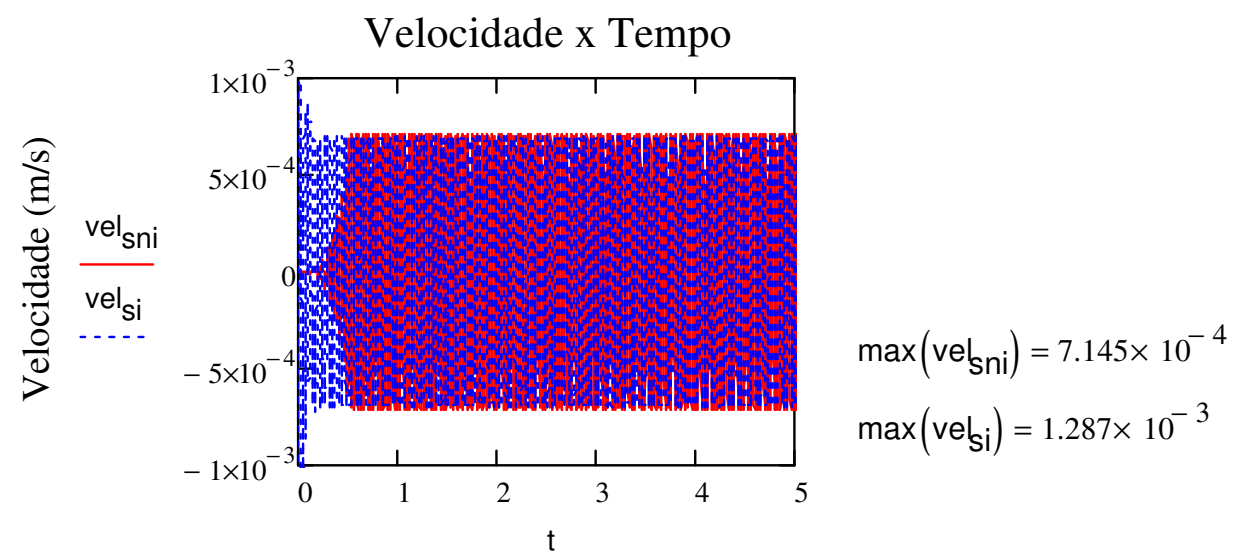

Tempo (s)

Gráfico 6.2 - Velocidades: sistema ideal e não-ideal $\operatorname{com} r \cdot \omega_{m}=6,3 \mathrm{~mm} / \mathrm{s}$ 


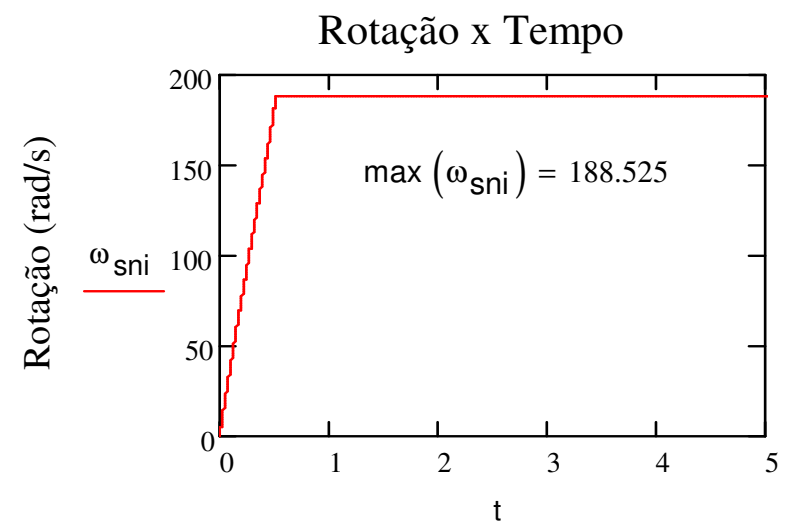

Tempo (s)

Gráfico 6.3 - Rotação do motor: sistema não-ideal com $r \cdot \omega_{m}=6,3 \mathrm{~mm} / \mathrm{s}$

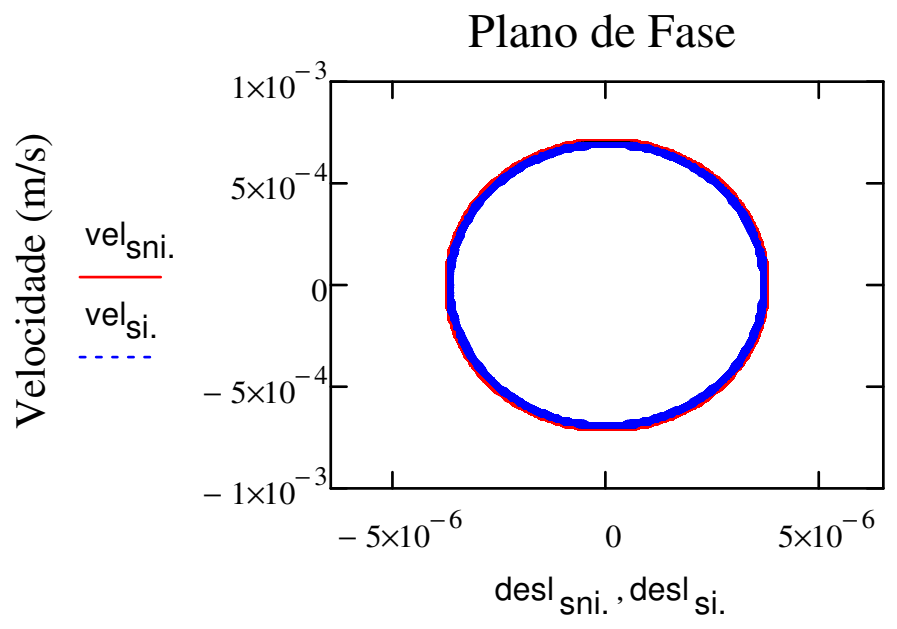

Deslocamento (m)

Gráfico 6.4 - Planos de fase: sistema ideal e não-ideal $\operatorname{com} r \cdot \omega_{m}=6,3 \mathrm{~mm} / \mathrm{s}$

6.2 Sistemas Não-Ideais com $r \cdot \omega_{m}=6,3 \mathrm{~mm} / \mathrm{s}, 2000 \mathrm{~mm} / \mathrm{s}$ e $4000 \mathrm{~mm} / \mathrm{s}$

Os resultados apresentados a seguir referem-se à análise, segundo a teoria de sistemas não-ideais de energia, da base de concreto com diferentes dimensões e altura variável. 


\subsubsection{Sistema Não-Ideal com b= 0.75, h=0.80 e L=variável $(\mathrm{m})$}

\section{Base de concreto com dimensões iniciais.}

Tabela 6.1 - Respostas do sistema não-ideal $b=0.75, h=0.80$ e $L=$ variável $(\mathrm{m})$

\begin{tabular}{ccccccccccccc}
\hline \multicolumn{2}{c}{ Caracteristicas da Estrutura } & $\begin{array}{c}\mathrm{r} 0 . \omega 0_{\mathrm{m}}: \\
\mathrm{r} 0:\end{array}$ & $\begin{array}{c}6,3 \\
0,034\end{array}$ & $\begin{array}{c}\mathrm{mm} / \mathrm{s} \\
\mathrm{mm}\end{array}$ & $\begin{array}{c}\mathrm{r} 1 . \omega 1_{\mathrm{m}}: \\
\mathrm{r} 1:\end{array}$ & $\begin{array}{c}2000 \\
10,67\end{array}$ & $\begin{array}{c}\mathrm{mm} / \mathrm{s} \\
\mathrm{mm}\end{array}$ & $\begin{array}{c}\mathrm{r} 2 . \omega 2_{\mathrm{m}}: \\
\mathrm{r} 2:\end{array}$ & $\begin{array}{c}4000 \\
21,34\end{array}$ & $\begin{array}{c}\mathrm{mm} / \mathrm{s} \\
\mathrm{mm}\end{array}$ \\
\hline $\mathrm{b}$ & $\mathrm{h}$ & $\mathrm{L}$ & $\omega$ & $\mathrm{E} 0$ & $\omega 0_{\mathrm{m}}$ & $v 0$ & $\mathrm{E} 1$ & $\omega 1_{\mathrm{m}}$ & $v 1$ & $\mathrm{E} 2$ & $\omega 2_{\mathrm{m}}$ & $v 2$ \\
$(\mathrm{~m})$ & $(\mathrm{m})$ & $(\mathrm{m})$ & $(\mathrm{rad} / \mathrm{s})$ & $(\mathrm{N} \cdot \mathrm{m})$ & $(\mathrm{rad} / \mathrm{s})$ & $(\mathrm{m})$ & $(\mathrm{N} \cdot \mathrm{m})$ & $(\mathrm{rad} / \mathrm{s})$ & $(\mathrm{m})$ & $(\mathrm{N} \cdot \mathrm{m})$ & $(\mathrm{rad} / \mathrm{s})$ & $(\mathrm{m})$ \\
\hline 0,75 & 0,80 & 1,50 & 670,43 & $3,67 \mathrm{E}-04$ & $1,89 \mathrm{E}+02$ & $1,39 \mathrm{E}-06$ & $3,70 \mathrm{E}+01$ & $1,89 \mathrm{E}+02$ & $4,40 \mathrm{E}-04$ & $1,48 \mathrm{E}+02$ & $1,89 \mathrm{E}+02$ & $8,82 \mathrm{E}-04$ \\
0,75 & 0,80 & 2,00 & 417,51 & $7,55 \mathrm{E}-04$ & $1,89 \mathrm{E}+02$ & $3,79 \mathrm{E}-06$ & $7,61 \mathrm{E}+01$ & $1,89 \mathrm{E}+02$ & $1,20 \mathrm{E}-03$ & $3,24 \mathrm{E}+02$ & $1,89 \mathrm{E}+02$ & $2,39 \mathrm{E}-03$ \\
0,75 & 0,80 & 2,50 & 287,37 & $2,23 \mathrm{E}-03$ & $1,89 \mathrm{E}+02$ & $1,04 \mathrm{E}-05$ & $2,25 \mathrm{E}+02$ & $1,89 \mathrm{E}+02$ & $3,26 \mathrm{E}-03$ & $9,00 \mathrm{E}+02$ & $1,89 \mathrm{E}+02$ & $6,47 \mathrm{E}-03$ \\
0,75 & 0,80 & 3,00 & 210,88 & $4,00 \mathrm{E}-02$ & $1,89 \mathrm{E}+02$ & $5,03 \mathrm{E}-05$ & $4,02 \mathrm{E}+03$ & $1,88 \mathrm{E}+02$ & $1,40 \mathrm{E}-02$ & $1,61 \mathrm{E}+04$ & $1,85 \mathrm{E}+02$ & $2,20 \mathrm{E}-02$ \\
0,75 & 0,80 & 3,50 & 161,81 & $5,00 \mathrm{E}-02$ & $1,89 \mathrm{E}+02$ & $8,55 \mathrm{E}-05$ & $5,00 \mathrm{E}+03$ & $1,59 \mathrm{E}+02$ & $2,70 \mathrm{E}-02$ & $2,00 \mathrm{E}+04$ & $1,47 \mathrm{E}+02$ & $2,60 \mathrm{E}-02$ \\
0,75 & 0,80 & 4,00 & 128,34 & $2,90 \mathrm{E}-02$ & $1,89 \mathrm{E}+02$ & $7,14 \mathrm{E}-05$ & $2,95 \mathrm{E}+03$ & $1,89 \mathrm{E}+02$ & $2,80 \mathrm{E}-02$ & $1,18 \mathrm{E}+04$ & $1,21 \mathrm{E}+02$ & $3,20 \mathrm{E}-02$ \\
0,75 & 0,80 & 4,50 & 104,41 & $1,80 \mathrm{E}-02$ & $1,89 \mathrm{E}+02$ & $6,34 \mathrm{E}-05$ & $1,84 \mathrm{E}+03$ & $1,89 \mathrm{E}+02$ & $2,00 \mathrm{E}-02$ & $7,36 \mathrm{E}+03$ & $1,04 \mathrm{E}+02$ & $3,90 \mathrm{E}-02$ \\
0,75 & 0,80 & 5,00 & 86,69 & $1,20 \mathrm{E}-02$ & $1,89 \mathrm{E}+02$ & $5,39 \mathrm{E}-05$ & $1,20 \mathrm{E}+03$ & $1,89 \mathrm{E}+02$ & $1,80 \mathrm{E}-02$ & $4,80 \mathrm{E}+03$ & $9,36 \mathrm{E}+01$ & $4,70 \mathrm{E}-02$ \\
0,75 & 0,80 & 5,50 & 73,18 & $8,02 \mathrm{E}-03$ & $1,89 \mathrm{E}+02$ & $4,38 \mathrm{E}-05$ & $8,08 \mathrm{E}+02$ & $1,89 \mathrm{E}+02$ & $1,40 \mathrm{E}-02$ & $3,23 \mathrm{E}+03$ & $1,90 \mathrm{E}+02$ & $3,40 \mathrm{E}-02$ \\
0,75 & 0,80 & 6,00 & 62,63 & $5,61 \mathrm{E}-03$ & $1,89 \mathrm{E}+02$ & $3,77 \mathrm{E}-05$ & $5,65 \mathrm{E}+02$ & $1,89 \mathrm{E}+02$ & $1,30 \mathrm{E}-02$ & $2,26 \mathrm{E}+03$ & $1,89 \mathrm{E}+02$ & $2,70 \mathrm{E}-02$ \\
\hline
\end{tabular}

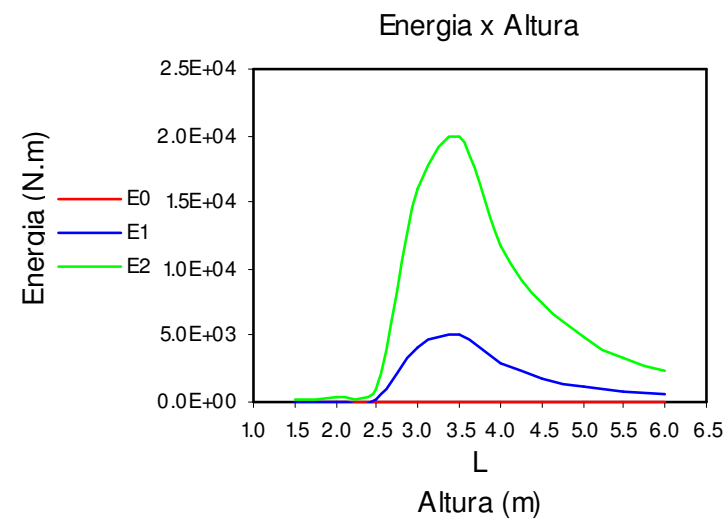

Gráfico 6.5 - Energia consumida pela estrutura x Altura

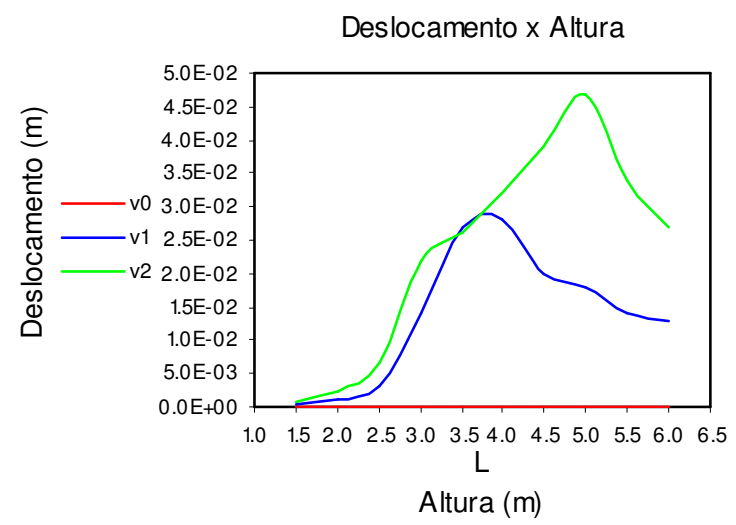

Gráfico 6.7 - Deslocamento da estrutura x Altura
Freqüência Natural \& Rotação Nominal

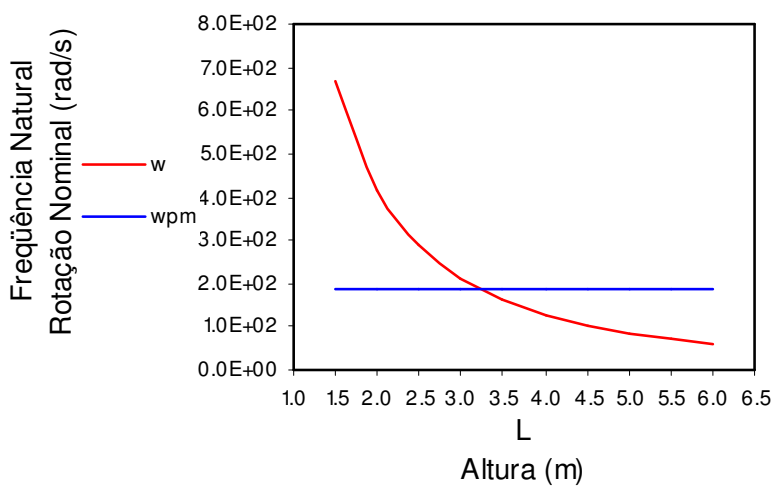

Gráfico 6.6 - Freqüência natural x Altura \& Rotação

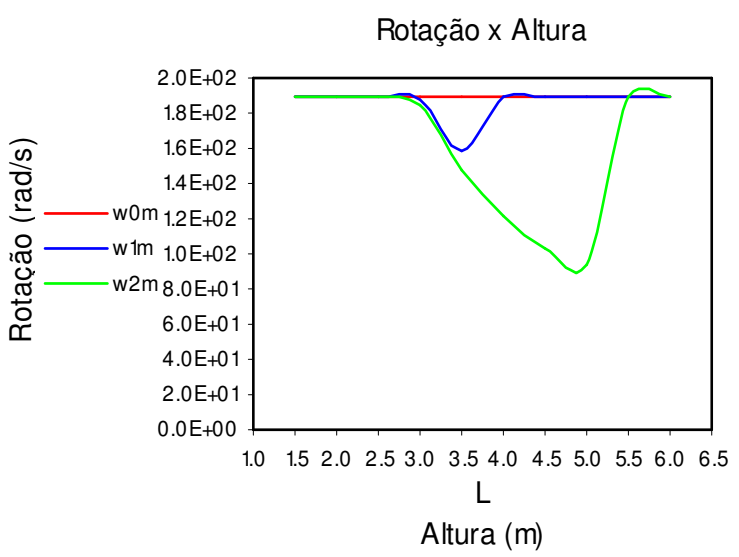

Gráfico 6.8 - Rotação do motor x Altura 


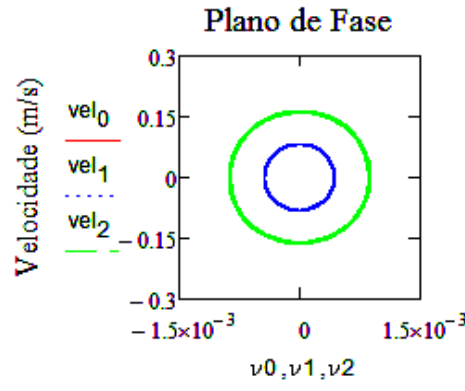

Deslocamento (m)

Gráfico 6.9 - Plano de fase para $L=1.5 \mathrm{~m}$

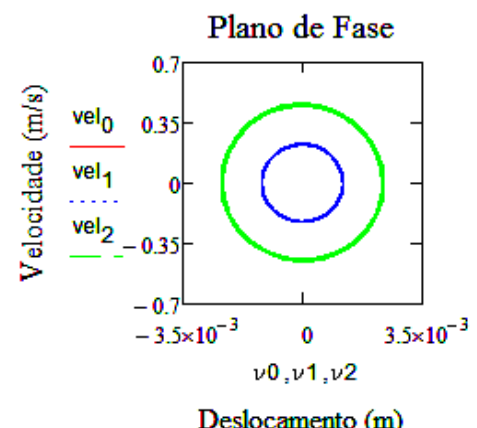

Gráfico 6.11 - Plano de fase para L=2.0m

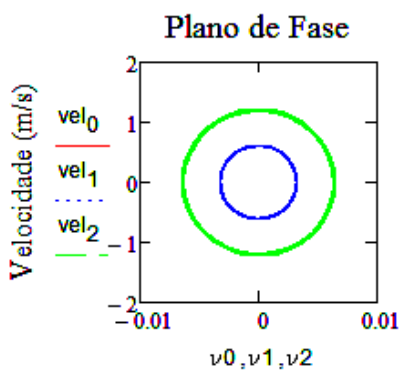

Deslocamento (m)

Gráfico 6.13 - Plano de fase para $L=2.5 \mathrm{~m}$

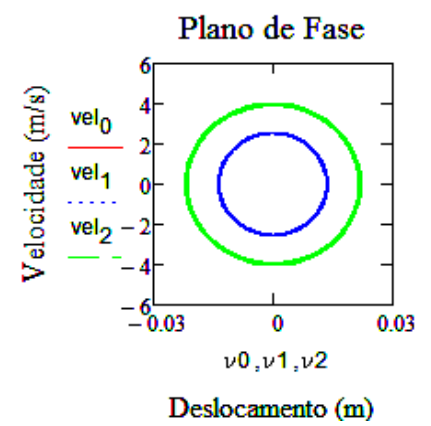

Gráfico 6.15 - Plano de fase para $L=3.0 \mathrm{~m}$

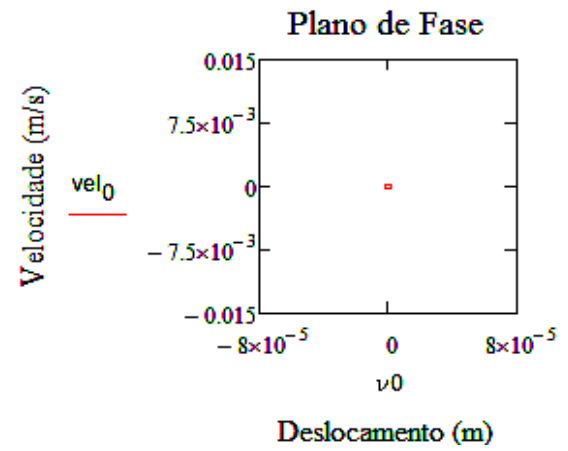

Gráfico 6.10 - Plano de fase para $L=1.5 \mathrm{~m}$ detalhe vel $_{0} \times$ vo

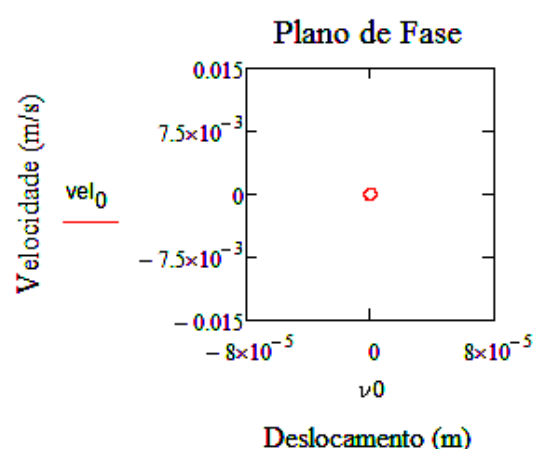

Gráfico 6.12 - Plano de fase para $L=2.0 \mathrm{~m}$ detalhe vel $_{0} \mathrm{x}$ vo

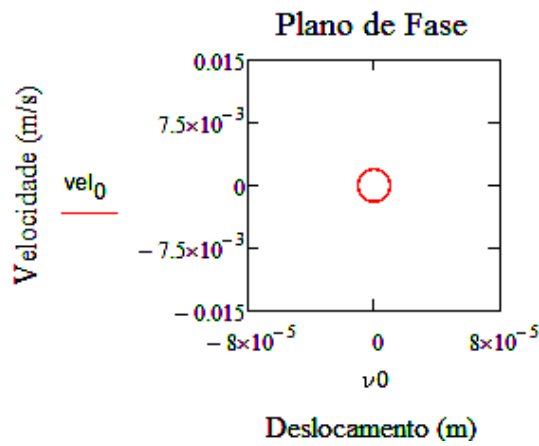

Gráfico 6.14 - Plano de fase para $L=2.5 \mathrm{~m}$ detalhe $\mathrm{vel}_{0} \times$ vo

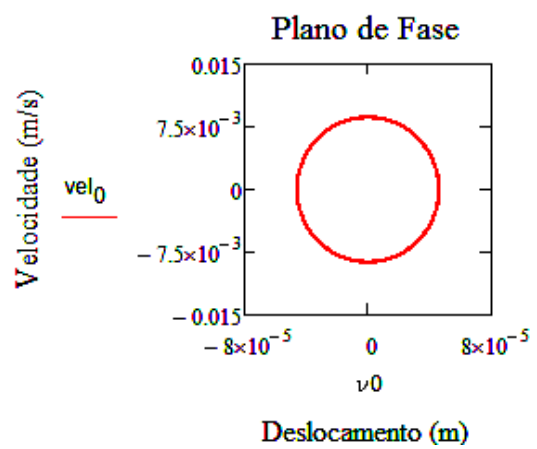

Gráfico 6.16 - Plano de fase para $L=3.0 \mathrm{~m}$ detalhe vel 0 vo 


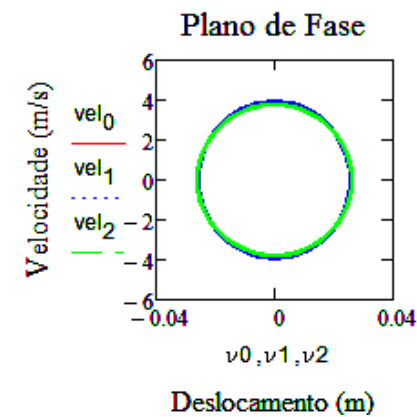

Gráfico 6.17 - Plano de fase para $L=3.5 \mathrm{~m}$

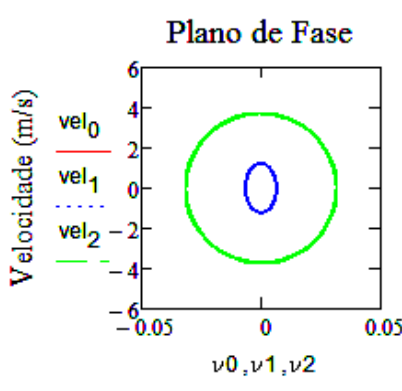

Deslocamento (m)

Gráfico 6.19 - Plano de fase para $L=4.0 \mathrm{~m}$

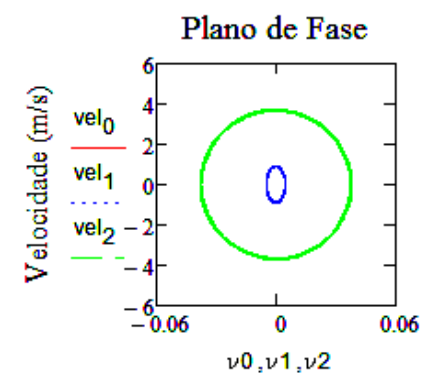

Deslocamento (m)

Gráfico 6.21 - Plano de fase para $L=4.5 \mathrm{~m}$

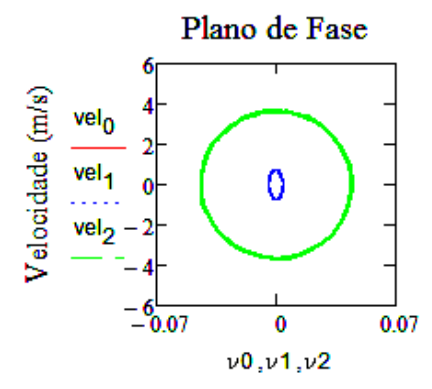

Deslocamento (m)

Gráfico 6.23 - Plano de fase para L=5.0m

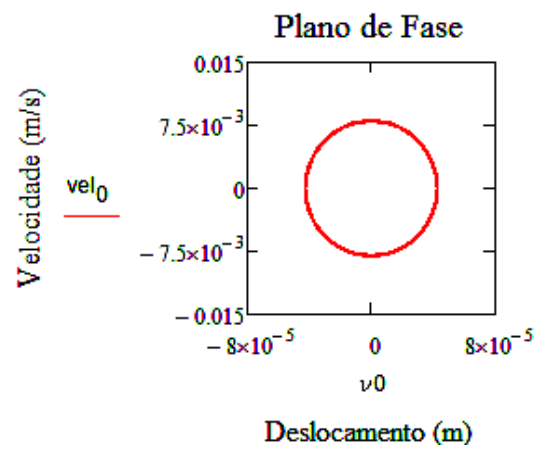

Gráfico 6.18 - Plano de fase para $L=3.5 \mathrm{~m}$ detalhe $\mathrm{vel}_{0} \mathrm{x}$ vo

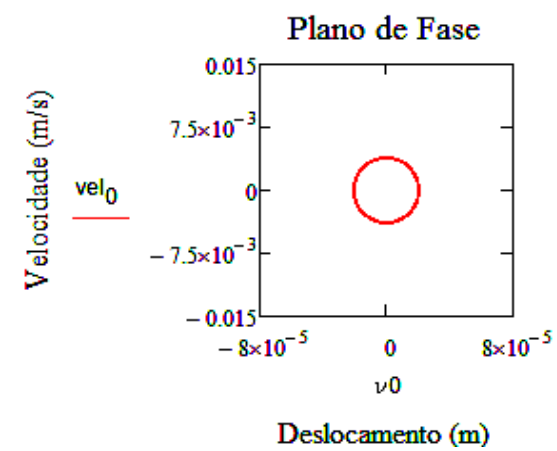

Gráfico 6.20 - Plano de fase para $L=4.0 \mathrm{~m}$ detalhe vel $x$ vo

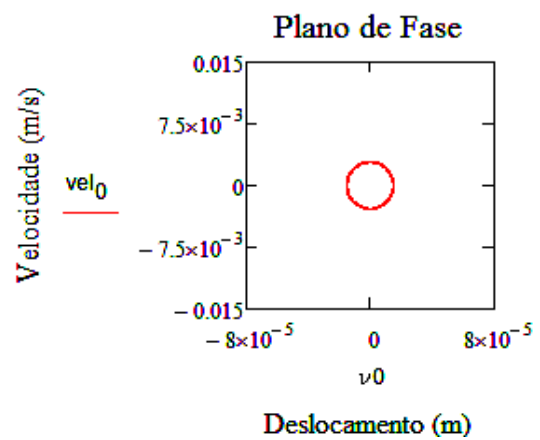

Gráfico 6.22 - Plano de fase para $L=4.5 \mathrm{~m}$ detalhe vel 0 vo

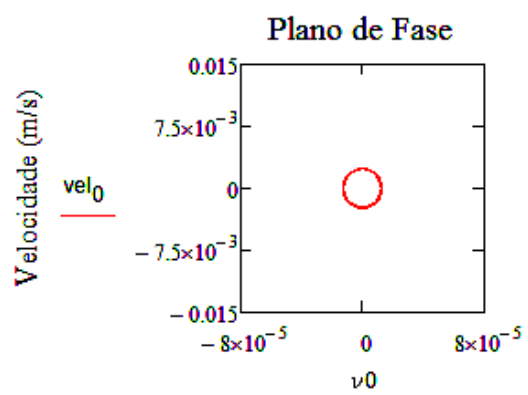

Deslocamento (m)

Gráfico 6.24 - Plano de fase para $L=5.0 \mathrm{~m}$ detalhe vel $_{0} \times$ vo 


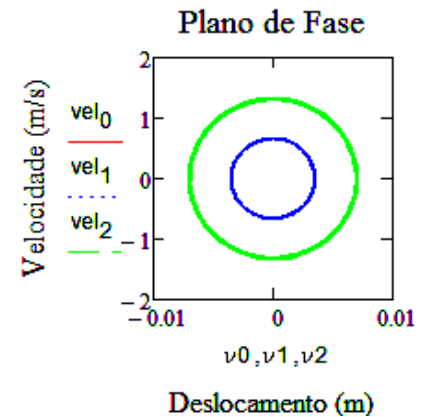

Gráfico 6.25 - Plano de fase para $L=5.5 \mathrm{~m}$

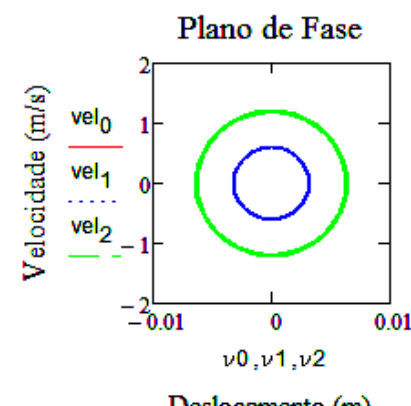

Gráfico 6.27 - Plano de fase para $L=6.0 \mathrm{~m}$

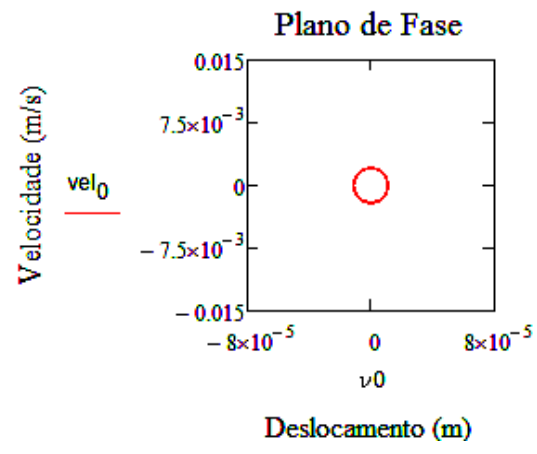

Gráfico 6.26 - Plano de fase para $L=5.5 \mathrm{~m}$ detalhe vel $_{0} \times$ vo

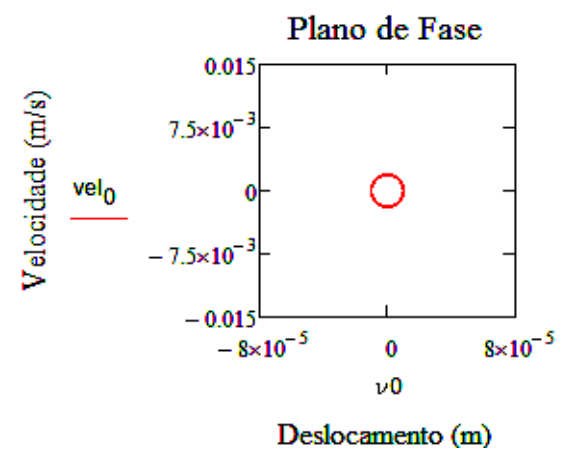

Gráfico 6.28 - Plano de fase para $L=6.0 \mathrm{~m}$ detalhe vel 0 vo 
6.2.2 Sistema Não-Ideal com $b=0.75, h=1.00$ e $L=$ variável $(m)$

\author{
Neste caso a base de concreto inicial é acrescida de $0.20 \mathrm{~m}$ na direção de maior \\ inércia.
}

Tabela 6.2 - Respostas do sistema não-ideal $b=0.75, h=1.00$ e $L=$ variável $(\mathrm{m})$

\begin{tabular}{ccccccccccccc}
\hline \multicolumn{2}{c}{ Caracteristicas da Estrutura } & $\begin{array}{c}\mathrm{r} 0 . \omega 0_{\mathrm{m}}: \\
\mathrm{r} 0:\end{array}$ & $\begin{array}{c}6.3 \\
0.034\end{array}$ & $\begin{array}{c}\mathrm{mm} / \mathrm{s} \\
\mathrm{mm}\end{array}$ & $\begin{array}{c}\mathrm{r} 1 . \omega 1_{\mathrm{m}}: \\
\mathrm{r} 1:\end{array}$ & $\begin{array}{c}2000 \\
10.67\end{array}$ & $\begin{array}{c}\mathrm{mm} / \mathrm{s} \\
\mathrm{mm}\end{array}$ & $\begin{array}{c}\mathrm{r} 2 . \omega 2_{\mathrm{m}}: \\
\mathrm{r} 2:\end{array}$ & $\begin{array}{c}4000 \\
21.34\end{array}$ & $\begin{array}{c}\mathrm{mm} / \mathrm{s} \\
\mathrm{mm}\end{array}$ \\
\hline $\mathrm{b}$ & $\mathrm{h}$ & $\mathrm{L}$ & $\omega$ & $\mathrm{E} 0$ & $\omega 0_{\mathrm{m}}$ & $v 0$ & $\mathrm{E} 1$ & $\omega 1_{\mathrm{m}}$ & $v 1$ & $\mathrm{E} 2$ & $\omega 2_{\mathrm{m}}$ & $v 2$ \\
$(\mathrm{~m})$ & $(\mathrm{m})$ & $(\mathrm{m})$ & $(\mathrm{rad} / \mathrm{s})$ & $(\mathrm{N} \cdot \mathrm{m})$ & $(\mathrm{rad} / \mathrm{s})$ & $(\mathrm{m})$ & $(\mathrm{N} \cdot \mathrm{m})$ & $(\mathrm{rad} / \mathrm{s})$ & $(\mathrm{m})$ & $(\mathrm{N} . \mathrm{m})$ & $(\mathrm{rad} / \mathrm{s})$ & $(\mathrm{m})$ \\
\hline 0.75 & 1.00 & 1.50 & 726.04 & $3.09 \mathrm{E}-04$ & $1.89 \mathrm{E}+02$ & $1.09 \mathrm{E}-06$ & $3.11 \mathrm{E}+01$ & $1.89 \mathrm{E}+02$ & $3.48 \mathrm{E}-04$ & $1.25 \mathrm{E}+02$ & $1.89 \mathrm{E}+02$ & $6.96 \mathrm{E}-04$ \\
0.75 & 1.00 & 2.00 & 449.02 & $6.00 \mathrm{E}-04$ & $1.89 \mathrm{E}+02$ & $2.93 \mathrm{E}-06$ & $6.05 \mathrm{E}+01$ & $1.89 \mathrm{E}+02$ & $9.29 \mathrm{E}-04$ & $2.42 \mathrm{E}+02$ & $1.89 \mathrm{E}+02$ & $1.85 \mathrm{E}-03$ \\
0.75 & 1.00 & 2.50 & 307.27 & $1.54 \mathrm{E}-03$ & $1.89 \mathrm{E}+02$ & $7.56 \mathrm{E}-06$ & $1.55 \mathrm{E}+02$ & $1.89 \mathrm{E}+02$ & $2.39 \mathrm{E}-03$ & $6.20 \mathrm{E}+02$ & $1.89 \mathrm{E}+02$ & $4.75 \mathrm{E}-03$ \\
0.75 & 1.00 & 3.00 & 224.36 & $1.30 \mathrm{E}-02$ & $1.89 \mathrm{E}+02$ & $2.90 \mathrm{E}-05$ & $1.31 \mathrm{E}+03$ & $1.89 \mathrm{E}+02$ & $8.86 \mathrm{E}-03$ & $5.23 \mathrm{E}+03$ & $1.88 \mathrm{E}+02$ & $1.50 \mathrm{E}-02$ \\
0.75 & 1.00 & 3.50 & 171.43 & $5.00 \mathrm{E}-02$ & $1.89 \mathrm{E}+02$ & $8.11 \mathrm{E}-05$ & $5.04 \mathrm{E}+03$ & $1.69 \mathrm{E}+02$ & $2.50 \mathrm{E}-02$ & $2.01 \mathrm{E}+04$ & $1.55 \mathrm{E}+02$ & $2.40 \mathrm{E}-02$ \\
0.75 & 1.00 & 4.00 & 135.46 & $2.90 \mathrm{E}-02$ & $1.89 \mathrm{E}+02$ & $6.78 \mathrm{E}-05$ & $2.93 \mathrm{E}+03$ & $1.89 \mathrm{E}+02$ & $2.60 \mathrm{E}-02$ & $1.17 \mathrm{E}+04$ & $1.27 \mathrm{E}+02$ & $2.80 \mathrm{E}-02$ \\
0.75 & 1.00 & 4.50 & 109.85 & $1.80 \mathrm{E}-02$ & $1.89 \mathrm{E}+02$ & $5.53 \mathrm{E}-05$ & $1.80 \mathrm{E}+03$ & $1.89 \mathrm{E}+02$ & $2.00 \mathrm{E}-02$ & $7.21 \mathrm{E}+03$ & $1.08 \mathrm{E}+02$ & $3.40 \mathrm{E}-02$ \\
0.75 & 1.00 & 5.00 & 90.94 & $1.20 \mathrm{E}-02$ & $1.89 \mathrm{E}+02$ & $4.79 \mathrm{E}-05$ & $1.16 \mathrm{E}+03$ & $1.89 \mathrm{E}+02$ & $1.50 \mathrm{E}-02$ & $4.65 \mathrm{E}+03$ & $9.75 \mathrm{E}+01$ & $4.10 \mathrm{E}-02$ \\
0.75 & 1.00 & 5.50 & 76.57 & $7.70 \mathrm{E}-03$ & $1.89 \mathrm{E}+02$ & $3.68 \mathrm{E}-05$ & $7.75 \mathrm{E}+02$ & $1.89 \mathrm{E}+02$ & $1.20 \mathrm{E}-02$ & $3.10 \mathrm{E}+03$ & $1.89 \mathrm{E}+02$ & $3.00 \mathrm{E}-02$ \\
0.75 & 1.00 & 6.00 & 65.38 & $5.31 \mathrm{E}-03$ & $1.89 \mathrm{E}+02$ & $3.56 \mathrm{E}-05$ & $5.35 \mathrm{E}+02$ & $1.89 \mathrm{E}+02$ & $1.10 \mathrm{E}-02$ & $2.14 \mathrm{E}+03$ & $1.89 \mathrm{E}+02$ & $2.40 \mathrm{E}-02$ \\
\hline
\end{tabular}

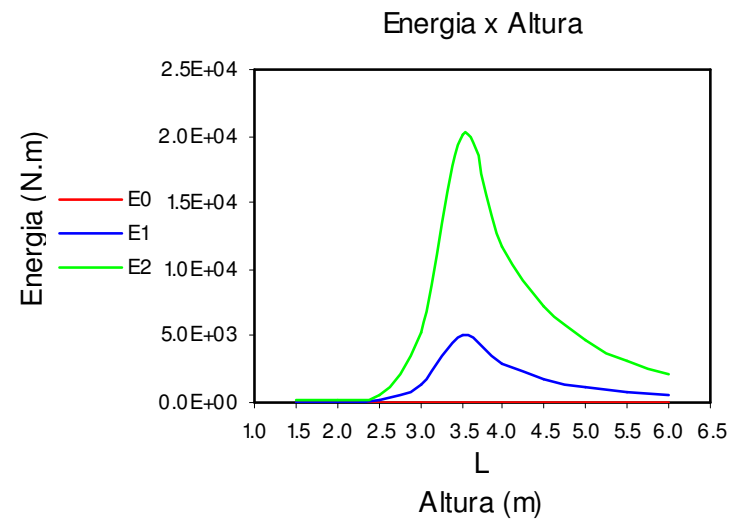

Gráfico 6.29 - Energia consumida pela estrutura x Altura
Freqüência Natural \& Rotação Nominal

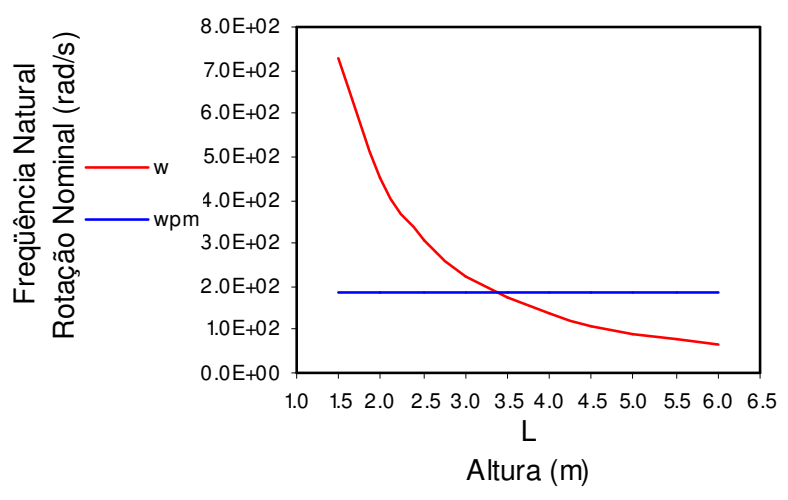

Gráfico 6.30 - Freqüência natural x Altura \& Rotação

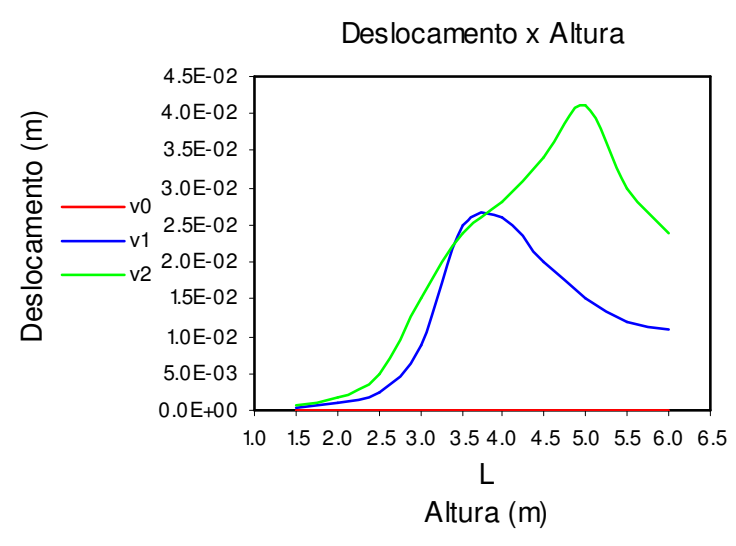

Gráfico 6.31 - Deslocamento da estrutura x Altura

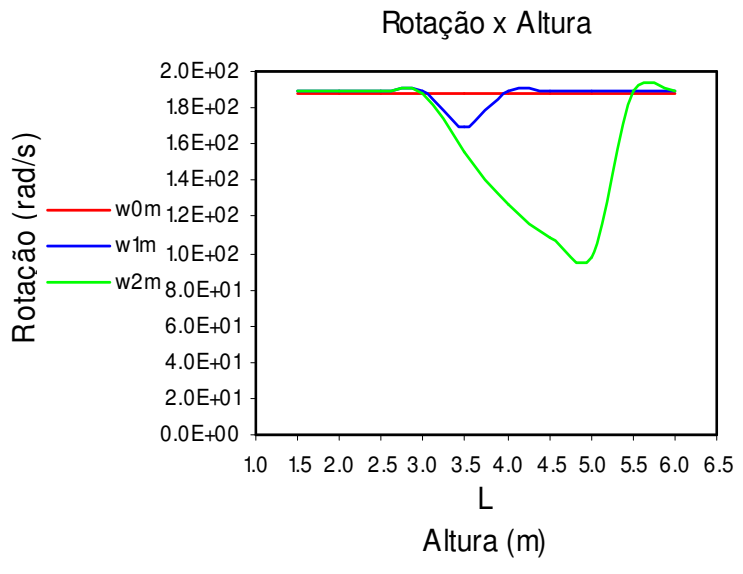

Gráfico 6.32 - Rotação do motor x Altura 


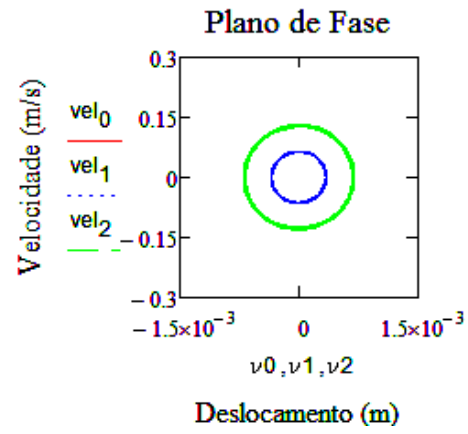

Gráfico 6.33 - Plano de fase para $L=1.5 \mathrm{~m}$

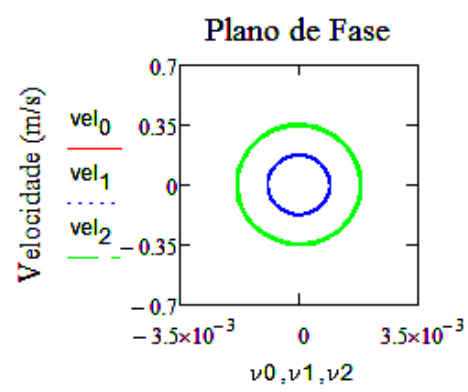

Deslocamento (m)

Gráfico 6.35 - Plano de fase para L=2.0m

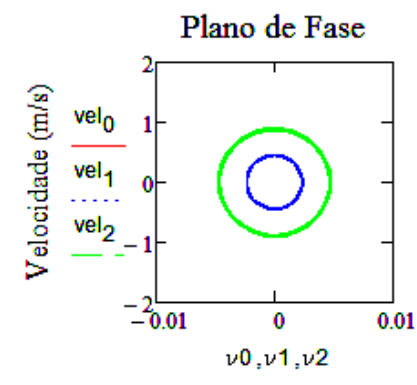

Deslocamento (m)

Gráfico 6.37 - Plano de fase para L=2.5m

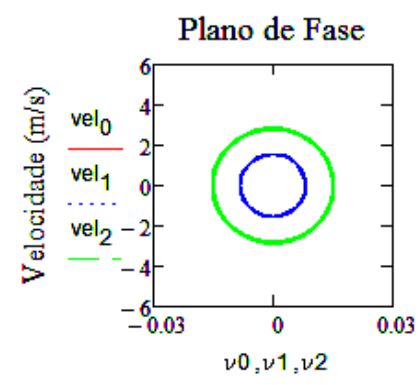

Deslocamento (m)

Gráfico 6.39 - Plano de fase para $L=3.0 \mathrm{~m}$

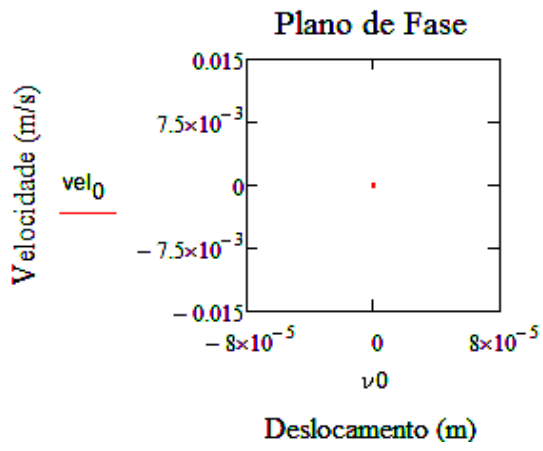

Gráfico 6.34 - Plano de fase para $L=1.5 \mathrm{~m}$ detalhe vel ${ }_{0} \times$ vo

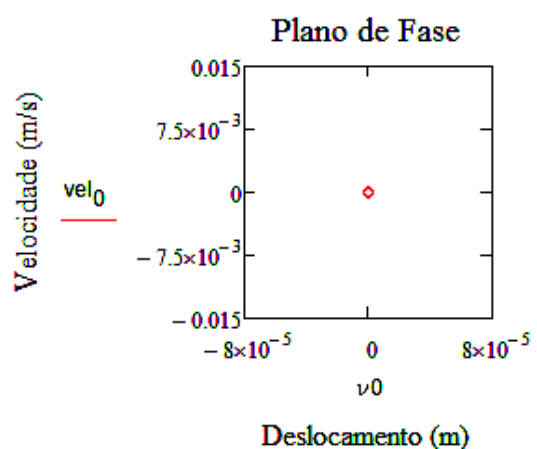

Gráfico 6.36 - Plano de fase para $L=2.0 \mathrm{~m}$ detalhe vel $\mathrm{x}_{0}$ vo

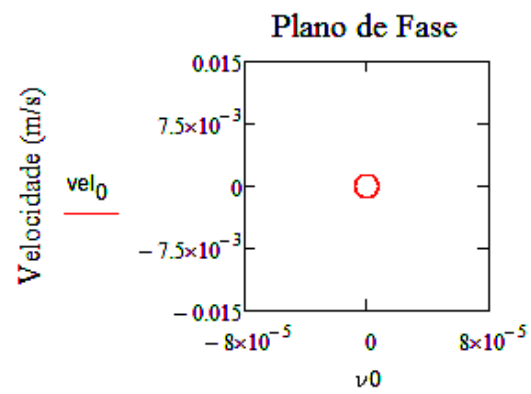

Deslocamento (m)

Gráfico 6.38 - Plano de fase para $L=2.5 \mathrm{~m}$ detalhe vel $x$ vo

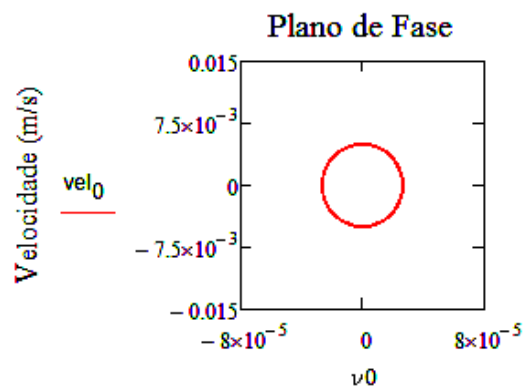

Deslocamento (m)

Gráfico 6.40 - Plano de fase para $L=3.0 \mathrm{~m}$ detalhe vel $x$ vo 


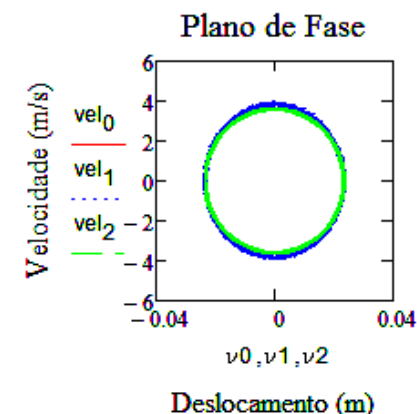

Gráfico 6.41 - Plano de fase para $L=3.5 \mathrm{~m}$

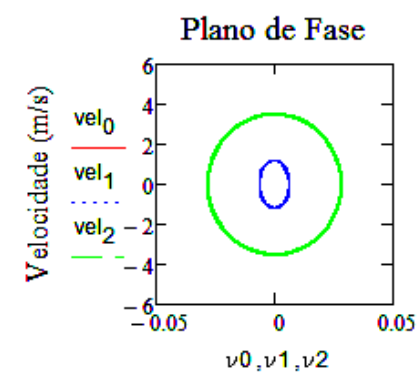

Deslocamento (m)

Gráfico 6.43 - Plano de fase para $L=4.0 \mathrm{~m}$

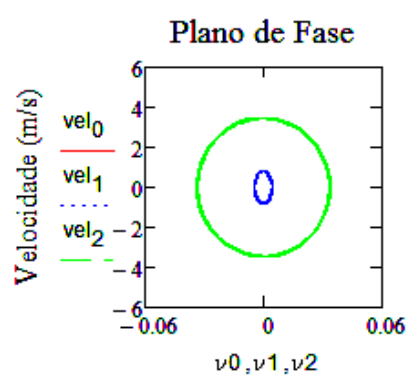

Deslocamento (m)

Gráfico 6.45 - Plano de fase para $L=4.5 \mathrm{~m}$

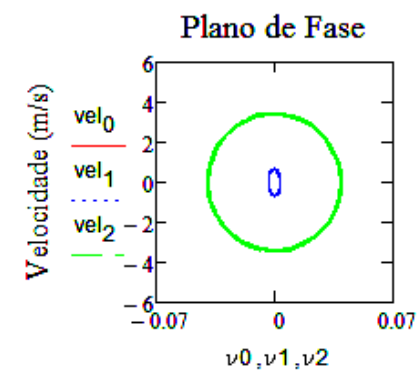

Deslocamento (m)

Gráfico 6.47 - Plano de fase para $L=5.0 \mathrm{~m}$

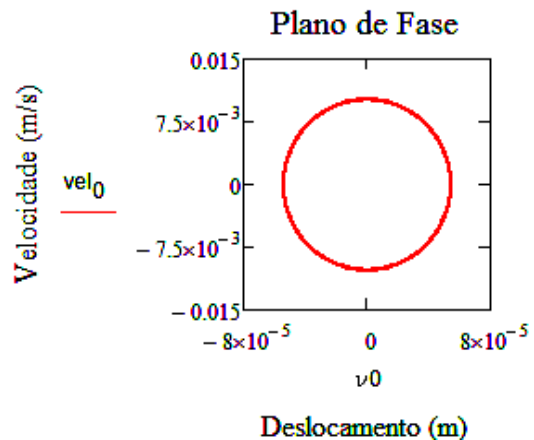

Gráfico 6.42 - Plano de fase para $L=3.5 \mathrm{~m}$ detalhe vel 0 vo

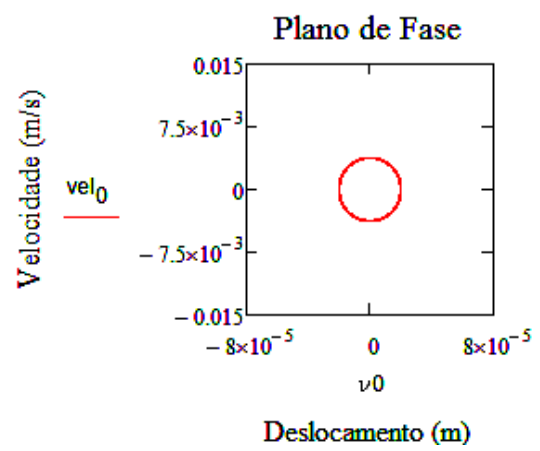

Gráfico 6.44 - Plano de fase para $L=4.0 \mathrm{~m}$ detalhe vel $_{0} \times$ vo

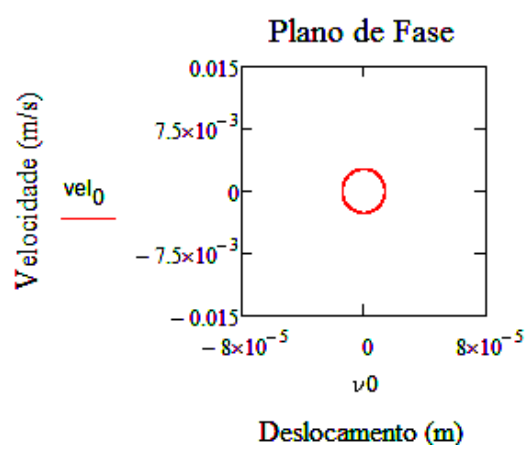

Gráfico 6.46 - Plano de fase para $L=4.5 \mathrm{~m}$ detalhe vel $x$ vo

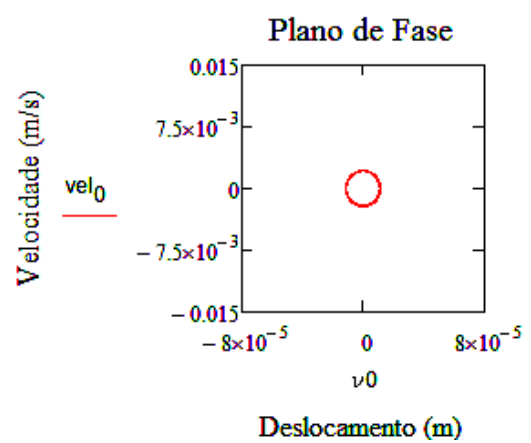

Gráfico 6.48 - Plano de fase para $L=5.0 \mathrm{~m}$ detalhe vel 0 vo 


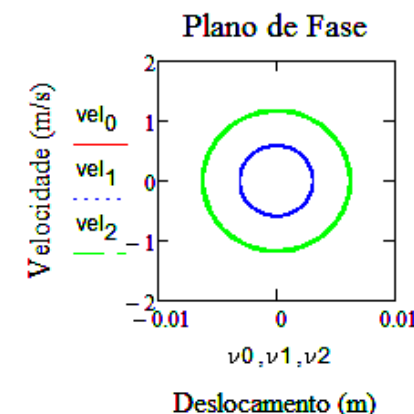

Gráfico 6.49 - Plano de fase para $L=5.5 \mathrm{~m}$

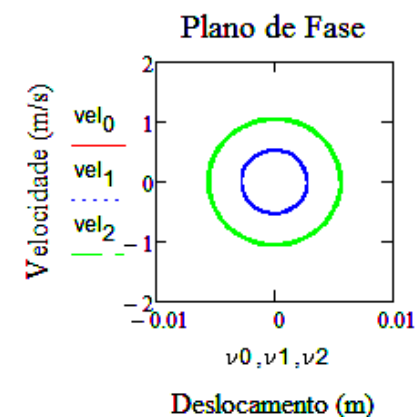

Gráfico 6.51 - Plano de fase para $L=6.0 \mathrm{~m}$

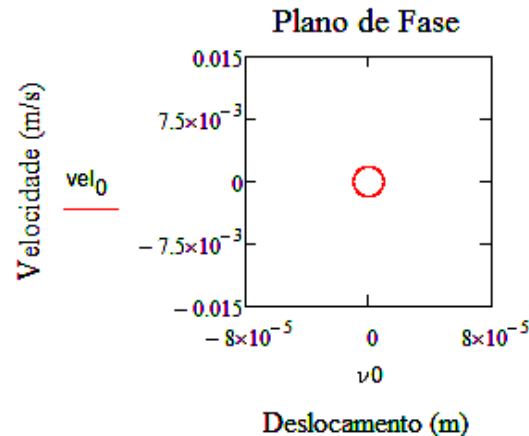

Gráfico 6.50 - Plano de fase para $L=5.5 \mathrm{~m}$ detalhe vel $_{0} \times$ vo

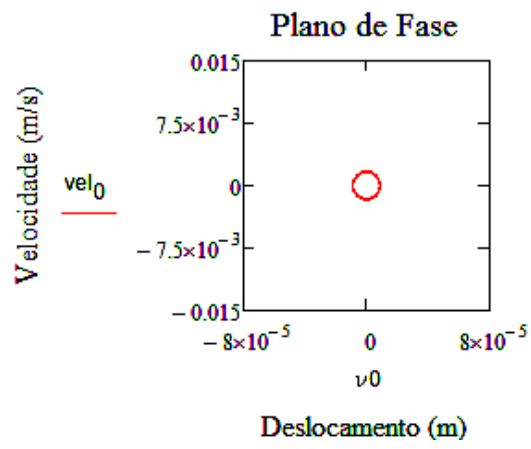

Gráfico 6.52 - Plano de fase para $L=6.0 \mathrm{~m}$ detalhe vel 0 vo 
6.2.3 Sistema Não-Ideal com $b=0.95, h=0.80$ e $L=$ variável $(m)$

Neste caso a base de concreto inicial é acrescida de $0.20 \mathrm{~m}$ na direção de menor inércia.

Tabela 6.3 - Respostas do sistema não-ideal $b=0.95, h=0.80$ e $\mathrm{L}=$ variável $(\mathrm{m})$

\begin{tabular}{|c|c|c|c|c|c|c|c|c|c|c|c|c|}
\hline \multicolumn{4}{|c|}{ Caracteristicas da Estrutura } & $\begin{array}{c}\mathrm{r} 0 . \omega 0_{\mathrm{m}} \\
\text { ro : }\end{array}$ & $\begin{array}{c}6.3 \\
0.034\end{array}$ & $\begin{array}{c}\mathrm{mm} / \mathrm{s} \\
\mathrm{mm}\end{array}$ & $\begin{array}{c}\mathrm{r} 1 . \omega 1_{\mathrm{m}}: \\
\mathrm{r} 1:\end{array}$ & $\begin{array}{l}2000 \\
10.67\end{array}$ & $\begin{array}{c}\mathrm{mm} / \mathrm{s} \\
\mathrm{mm}\end{array}$ & $\begin{array}{c}\text { r2. } \omega 2_{m}: \\
\text { r2: }\end{array}$ & $\begin{array}{l}4000 \\
21.34\end{array}$ & $\begin{array}{c}\mathrm{mm} / \mathrm{s} \\
\mathrm{mm}\end{array}$ \\
\hline $\begin{array}{c}\mathrm{b} \\
(\mathrm{m})\end{array}$ & $\begin{array}{c}\mathrm{h} \\
(\mathrm{m})\end{array}$ & $\begin{array}{l}\mathrm{L} \\
(\mathrm{m})\end{array}$ & $\begin{array}{c}\omega \\
(\mathrm{rad} / \mathrm{s})\end{array}$ & $\begin{array}{c}\text { E0 } \\
\text { (N.m) }\end{array}$ & $\begin{array}{c}\mathrm{\omega}_{\mathrm{m}} \\
(\mathrm{rad} / \mathrm{s})\end{array}$ & $\begin{array}{l}v 0 \\
(\mathrm{~m})\end{array}$ & $\begin{array}{c}\text { E1 } \\
\text { (N.m) }\end{array}$ & $\begin{array}{c}\omega 1_{\mathrm{m}} \\
(\mathrm{rad} / \mathrm{s})\end{array}$ & $\begin{array}{l}v 1 \\
(\mathrm{~m})\end{array}$ & $\begin{array}{c}\text { E2 } \\
\text { (N.m) }\end{array}$ & $\begin{array}{c}\mathrm{\omega} 2_{\mathrm{m}} \\
(\mathrm{rad} / \mathrm{s})\end{array}$ & $\begin{array}{l}v 2 \\
(\mathrm{~m})\end{array}$ \\
\hline 0.95 & 0.80 & 1.50 & 923.86 & $2.27 \mathrm{E}-04$ & $1.89 \mathrm{E}+02$ & $6.56 \mathrm{E}-07$ & $2.29 \mathrm{E}+01$ & $1.89 \mathrm{E}+02$ & $2.08 \mathrm{E}-04$ & $9.17 \mathrm{E}+01$ & $1.89 \mathrm{E}+02$ & $4.16 \mathrm{E}-04$ \\
\hline 0.95 & 0.80 & 2.00 & 571.12 & 3.93E-04 & $1.89 \mathrm{E}+02$ & 1.67E-06 & $3.96 \mathrm{E}+01$ & $1.89 \mathrm{E}+02$ & 5.30E-04 & $1.58 \mathrm{E}+02$ & $1.89 \mathrm{E}+02$ & $.06 \mathrm{E}-03$ \\
\hline 0.95 & 0.80 & 2.50 & 390.69 & 7.39E-04 & $1.89 \mathrm{E}+02$ & $3.78 \mathrm{E}-06$ & $7.45 \mathrm{E}+01$ & $1.89 \mathrm{E}+02$ & $1.20 \mathrm{E}-03$ & $2.98 \mathrm{E}+02$ & $1.89 \mathrm{E}+02$ & $2.40 \mathrm{E}-03$ \\
\hline 0.95 & 0.80 & 3.00 & 285.19 & 1.94E-03 & $1.89 \mathrm{E}+02$ & 8.90E-06 & $1.95 \mathrm{E}+02$ & $1.89 \mathrm{E}+02$ & 2.80E-03 & $7.81 \mathrm{E}+02$ & $1.89 \mathrm{E}+02$ & $5.58 \mathrm{E}-03$ \\
\hline 0.95 & 0.80 & 3.50 & 217.85 & $1.90 \mathrm{E}-02$ & $1.89 \mathrm{E}+02$ & 3.30E-05 & $1.87 \mathrm{E}+03$ & $1.89 \mathrm{E}+02$ & $9.79 \mathrm{E}-03$ & $7.49 \mathrm{E}+03$ & $1.87 \mathrm{E}+02$ & $1.70 \mathrm{E}-02$ \\
\hline 0.95 & 0.80 & 4.00 & 172.10 & 4.70E-02 & $1.89 \mathrm{E}+02$ & $7.60 \mathrm{E}-05$ & $4.70 \mathrm{E}+03$ & $1.71 \mathrm{E}+02$ & 2.40E-02 & $1.88 \mathrm{E}+04$ & $1.57 \mathrm{E}+02$ & $2.30 \mathrm{E}-02$ \\
\hline 0.95 & 0.80 & 4.50 & 139.54 & $2.90 \mathrm{E}-02$ & $1.89 \mathrm{E}+02$ & $6.16 \mathrm{E}-05$ & $2.89 \mathrm{E}+03$ & $1.89 \mathrm{E}+02$ & $2.50 \mathrm{E}-02$ & $1.16 \mathrm{E}+04$ & $1.31 \mathrm{E}+02$ & $2.70 \mathrm{E}-02$ \\
\hline 0.95 & 0.80 & 5.00 & 115.50 & $1.80 \mathrm{E}-02$ & $1.89 \mathrm{E}+02$ & 5.36E-05 & $1.86 \mathrm{E}+03$ & $1.89 \mathrm{E}+02$ & $1.80 \mathrm{E}-02$ & $7.44 \mathrm{E}+03$ & $1.13 E+02$ & $3.20 \mathrm{E}-02$ \\
\hline 0.95 & 0.80 & 5.50 & .23 & $1.20 \mathrm{E}-02$ & $1.89 \mathrm{E}+02$ & 4.30E-05 & $1.24 \mathrm{E}+03$ & $1.89 \mathrm{E}+02$ & 1.50E-02 & $4.97 \mathrm{E}+03$ & $1.02 \mathrm{E}+02$ & 3.70E-02 \\
\hline 0.95 & 0.80 & 6.00 & 83.01 & 8.50E-03 & $1.89 \mathrm{E}+02$ & 3.91E-05 & $8.56 \mathrm{E}+02$ & $1.89 \mathrm{E}+02$ & $1.30 \mathrm{E}-02$ & $3.43 E+03$ & $1.90 \mathrm{E}+02$ & 3.00E-02 \\
\hline
\end{tabular}

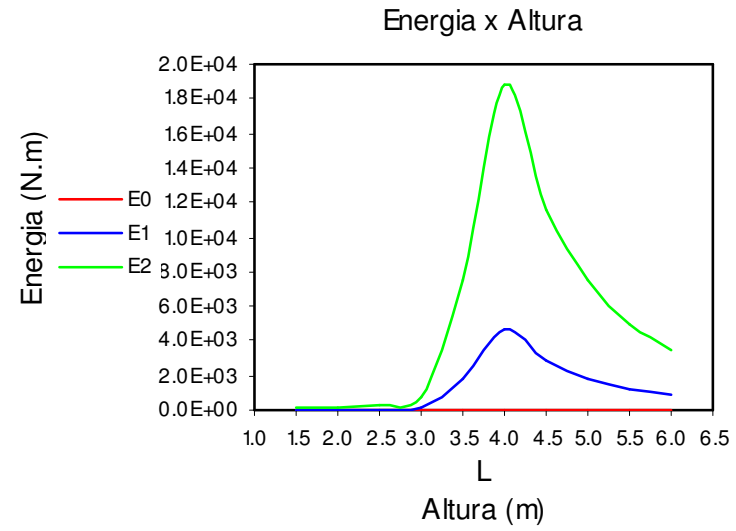

Gráfico 6.53 - Energia consumida pela estrutura x Altura

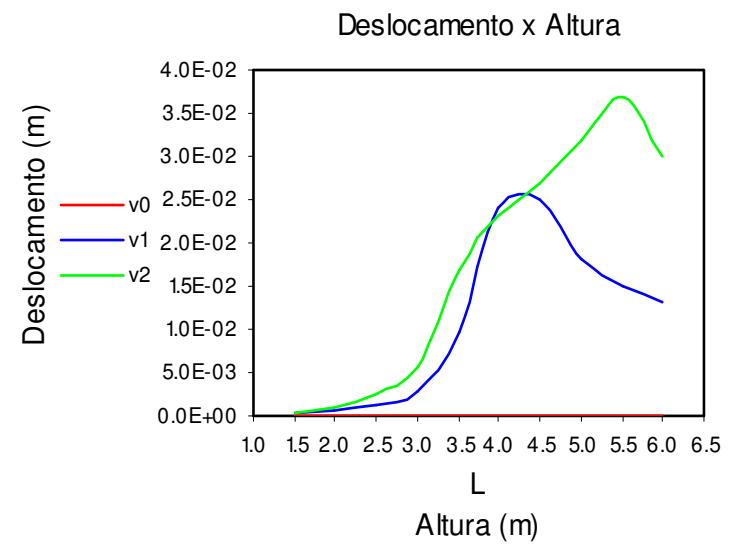

Gráfico 6.55 - Deslocamento da estrutura x Altura
Freqüência Natural \& Rotação Nominal

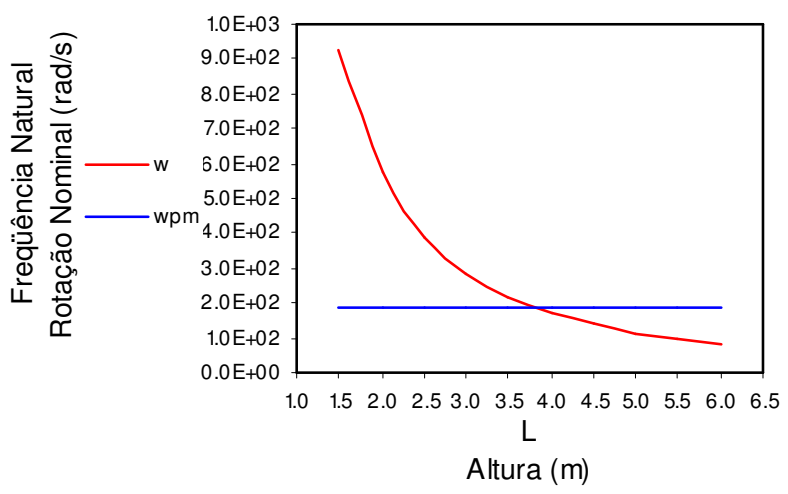

Gráfico 6.54 - Freqüência natural x Altura \& Rotação

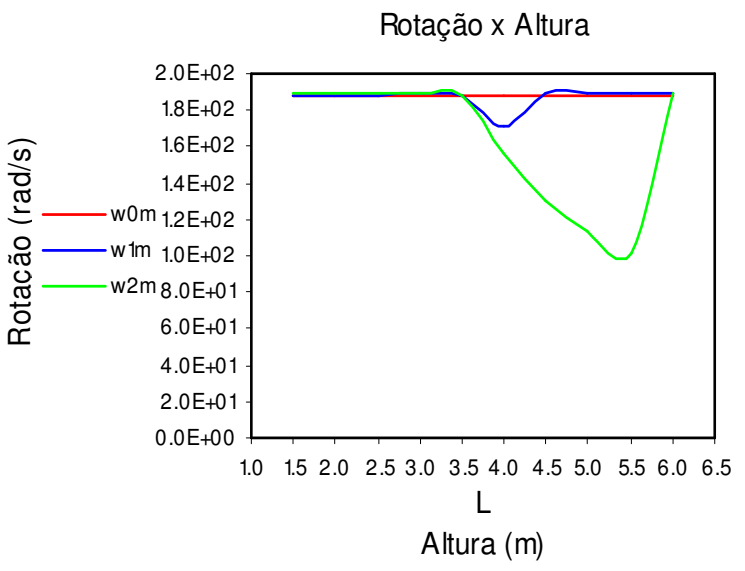

Gráfico 6.56 - Rotação do motor x Altura 


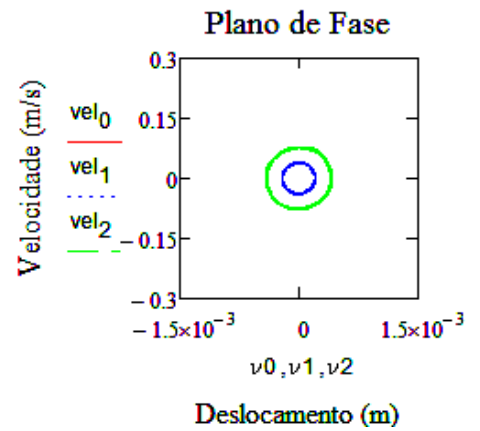

Gráfico 6.57 - Plano de fase para $L=1.5 \mathrm{~m}$

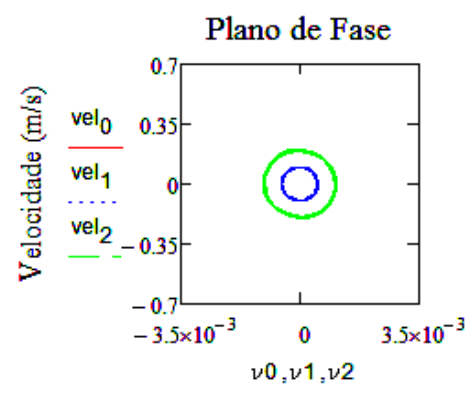

Deslocamento (m)

Gráfico 6.59 - Plano de fase para L=2.0m

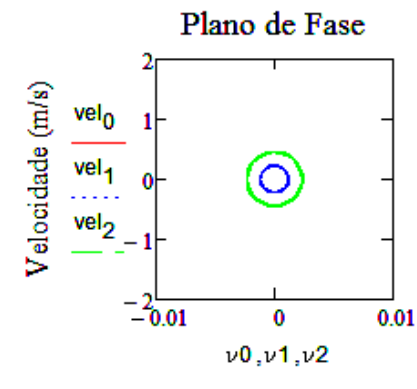

Deslocamento (m)

Gráfico 6.61 - Plano de fase para L=2.5m

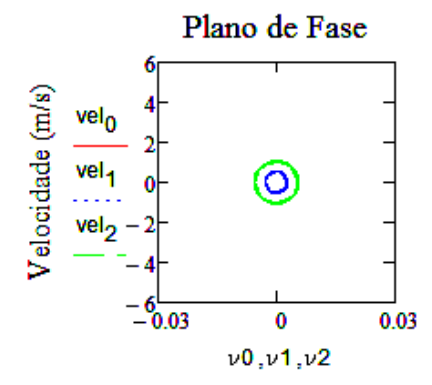

Deslocamento (m)

Gráfico 6.63 - Plano de fase para $L=3.0 \mathrm{~m}$

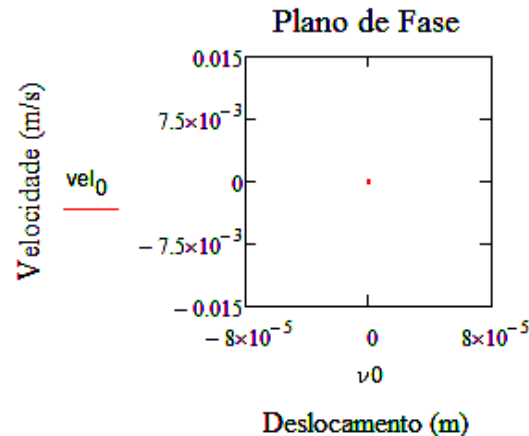

Gráfico 6.58 - Plano de fase para $L=1.5 \mathrm{~m}$ detalhe vel $_{0} \times$ vo

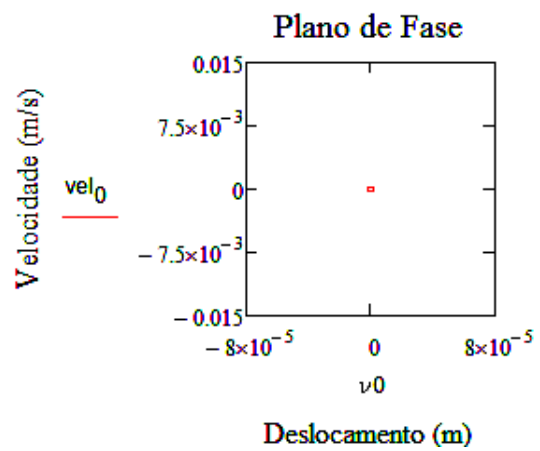

Gráfico 6.60 - Plano de fase para $L=2.0 \mathrm{~m}$ detalhe vel 0 vo

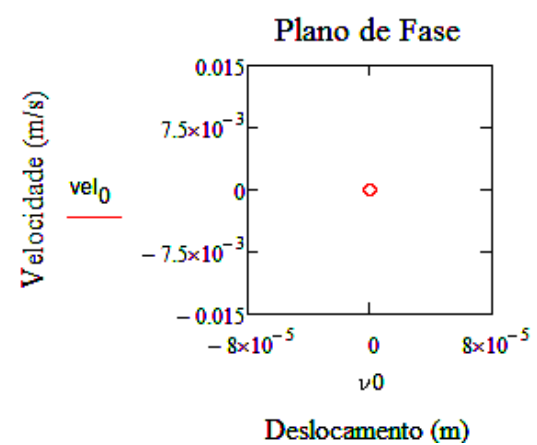

Gráfico 6.62 - Plano de fase para $L=2.5 \mathrm{~m}$ detalhe vel $_{0} \times$ vo

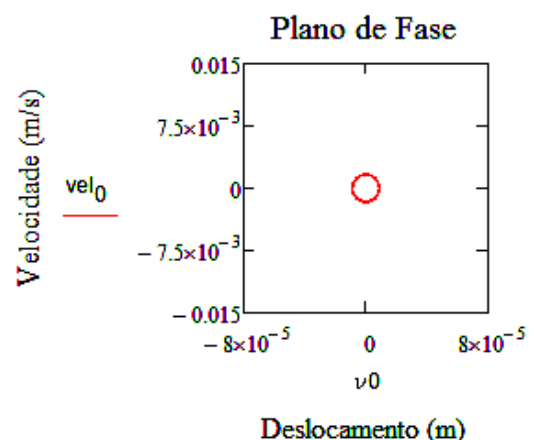

Gráfico 6.64 - Plano de fase para $L=3.0 \mathrm{~m}$ detalhe vel $x$ vo 


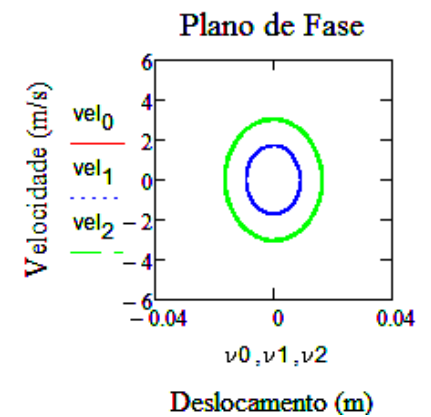

Gráfico 6.65 - Plano de fase para $L=3.5 \mathrm{~m}$

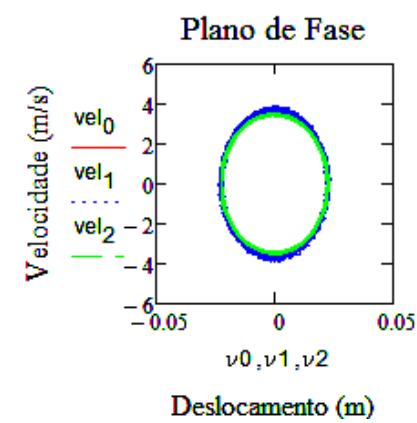

Gráfico 6.67 - Plano de fase para $L=4.0 \mathrm{~m}$

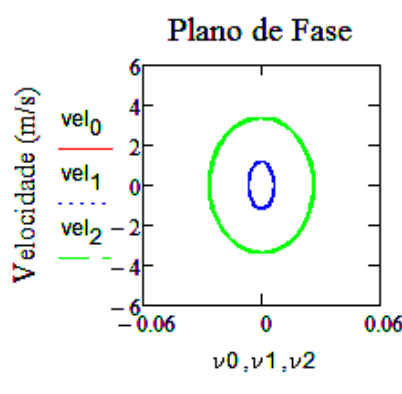

Deslocamento (m)

Gráfico 6.69 - Plano de fase para $L=4.5 \mathrm{~m}$

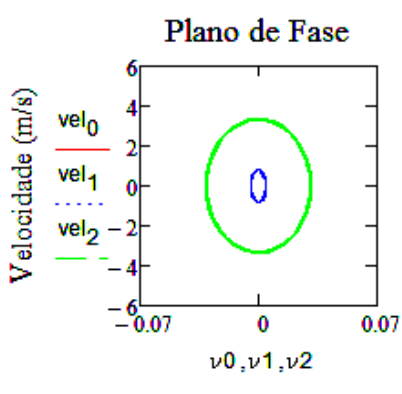

Deslocamento (m)

Gráfico 6.71 - Plano de fase para $L=5.0 \mathrm{~m}$

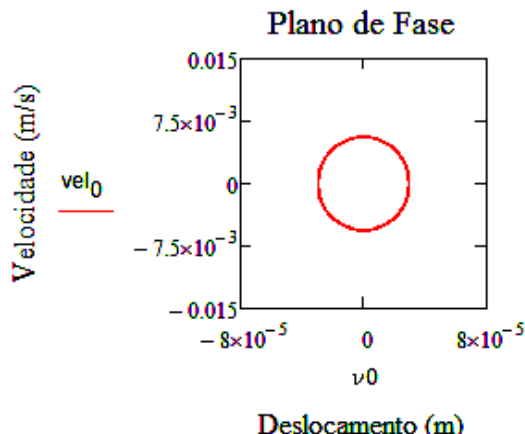

Gráfico 6.66 - Plano de fase para $L=3.5 \mathrm{~m}$ detalhe vel $_{0} \times$ vo

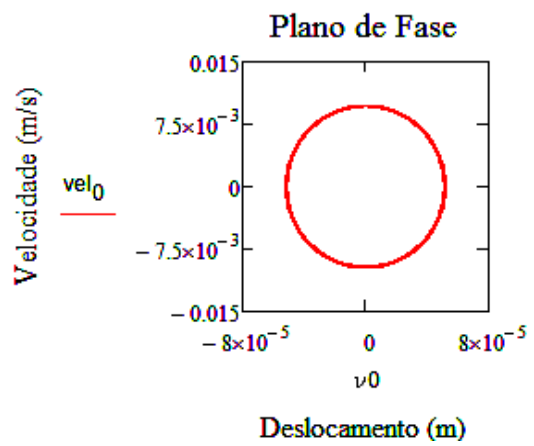

Gráfico 6.68 - Plano de fase para $L=4.0 \mathrm{~m}$ detalhe vel 0 vo

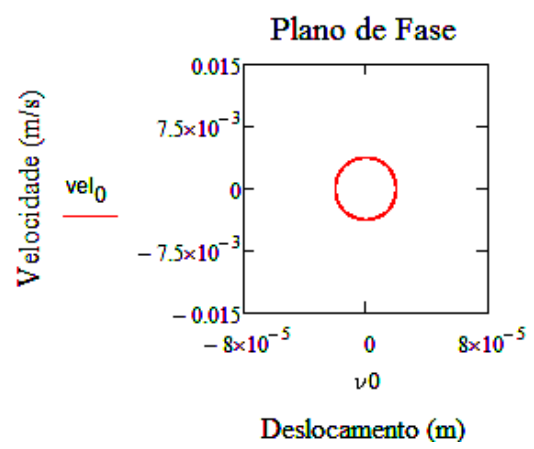

Gráfico 6.70 - Plano de fase para $L=4.5 \mathrm{~m}$ detalhe vel $_{0} \times$ vo

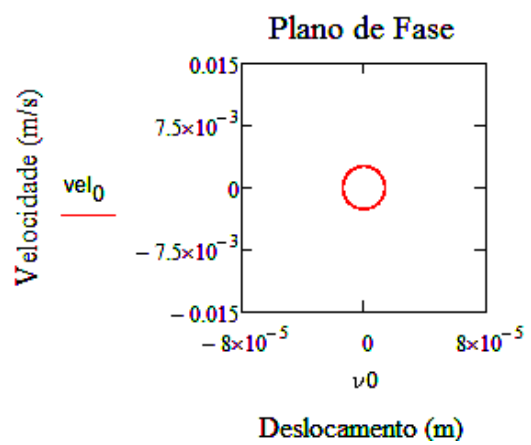

Gráfico 6.72 - Plano de fase para $L=5.0 \mathrm{~m}$ detalhe vel ${ }_{0} \times$ vo 


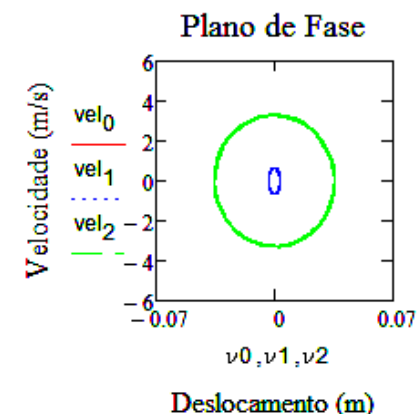

Gráfico 6.73 - Plano de fase para $L=5.5 \mathrm{~m}$

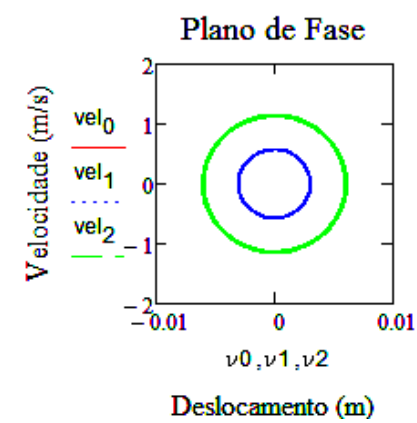

Gráfico 6.75 - Plano de fase para $L=6.0 \mathrm{~m}$

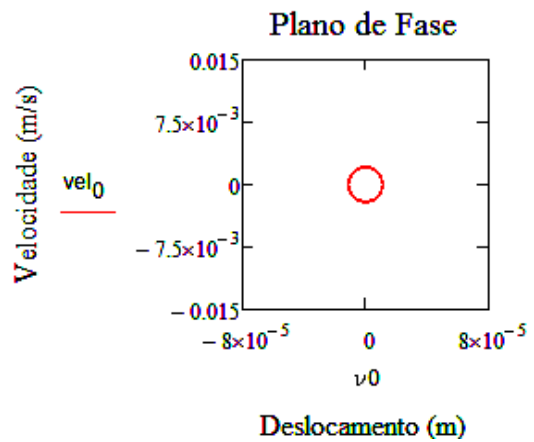

Gráfico 6.74 - Plano de fase para $L=5.5 \mathrm{~m}$ detalhe vel $_{0} \times$ vo

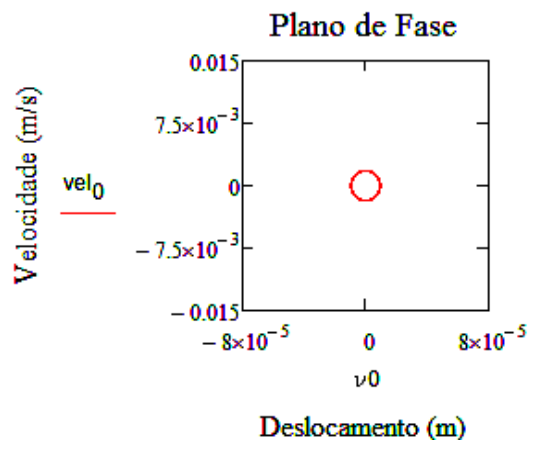

Gráfico 6.76 - Plano de fase para $L=6.0 \mathrm{~m}$ detalhe vel $_{0} \times$ vo

6.3 Sistemas Não-Ideais com $r \cdot \omega_{m}=6,3 \mathrm{~mm} / \mathrm{s}, 2000 \mathrm{~mm} / \mathrm{s}$ e $4000 \mathrm{~mm} / \mathrm{s}$ para diferentes bases

Os resultados apresentados a seguir referem-se à análise, segundo a teoria de sistemas não-ideais de energia, de três diferentes bases de concreto com altura variável. 
Energia Consumida E0 x Altura

ro. $\omega 0 \mathrm{~m}: 6.3 \mathrm{~mm} / \mathrm{s}$ (r0: $0.034 \mathrm{~mm})$

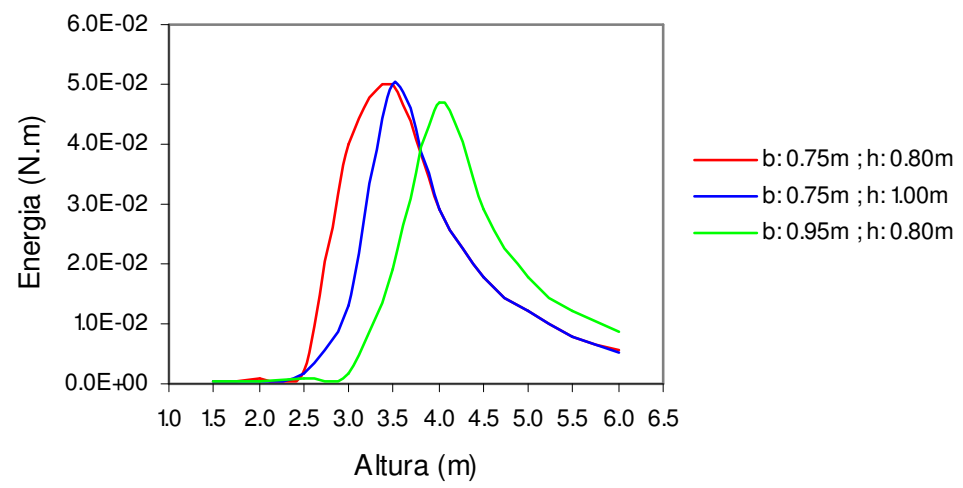

Gráfico 6.77 - Energia consumida E0 x Altura (r0.w0m: 6.3mm/s)

Energia Consumida E1 x Altura

r1. $\omega 1 \mathrm{~m}: 2000 \mathrm{~mm} / \mathrm{s}$ (r1: $10.67 \mathrm{~mm})$

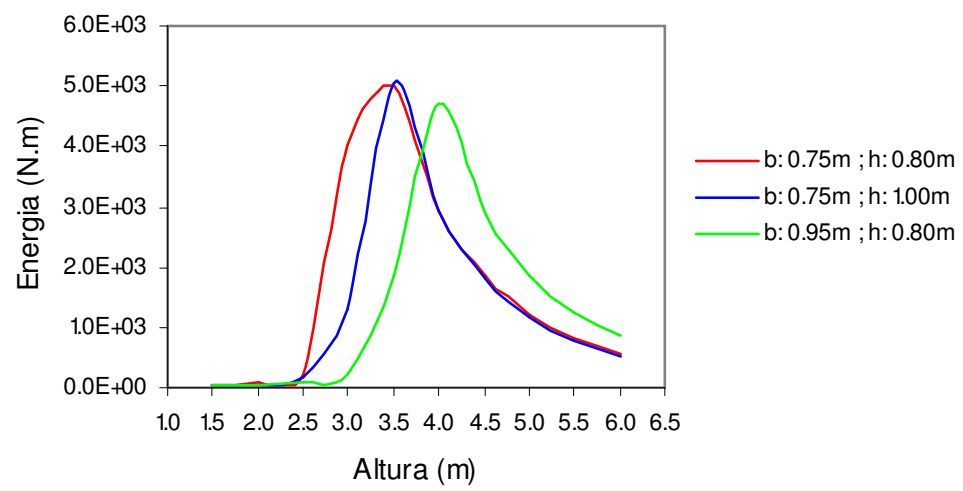

Gráfico 6.78 - Energia consumida E1 x Altura (r1.w1m: 2000mm/s)

Energia Consumida E2 x Altura

r2. $\omega 2 \mathrm{~m}: 4000 \mathrm{~mm} / \mathrm{s}$ (r2: $21.34 \mathrm{~mm})$

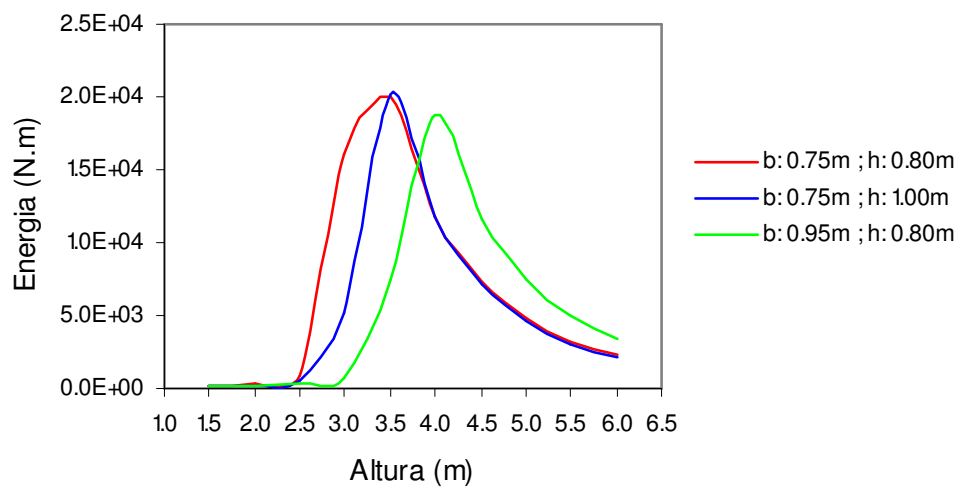

Gráfico 6.79 - Energia consumida E2 x Altura (r2.w2m: 4000mm/s) 
Rotação w0m x Altura

r0. $\omega 0 \mathrm{~m}: 6.3 \mathrm{~mm} / \mathrm{s}$ (r0: $0.034 \mathrm{~mm})$

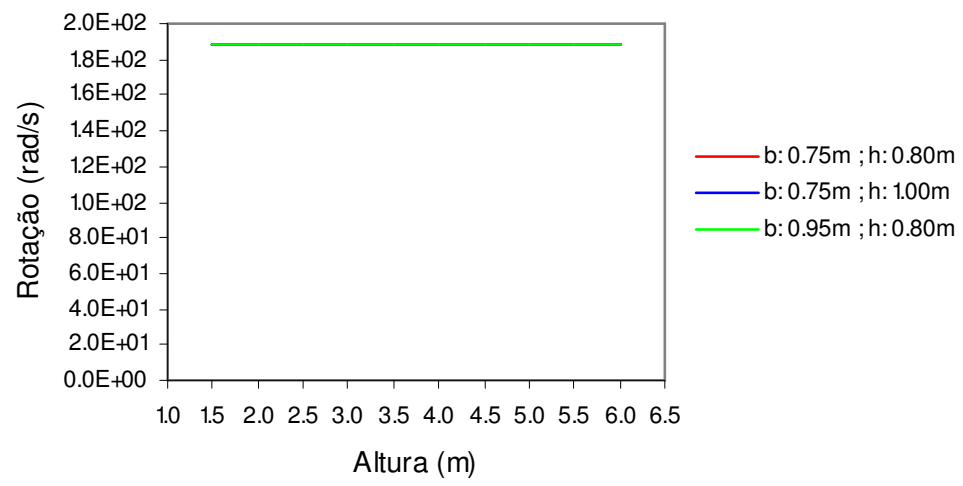

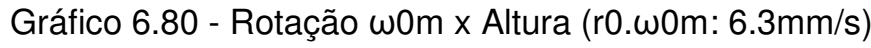

Rotação $\omega 1 \mathrm{~m} \times$ Altura

r1. $\omega 1 \mathrm{~m}: 2000 \mathrm{~mm} / \mathrm{s}$ (r1: $10.67 \mathrm{~mm}$ )

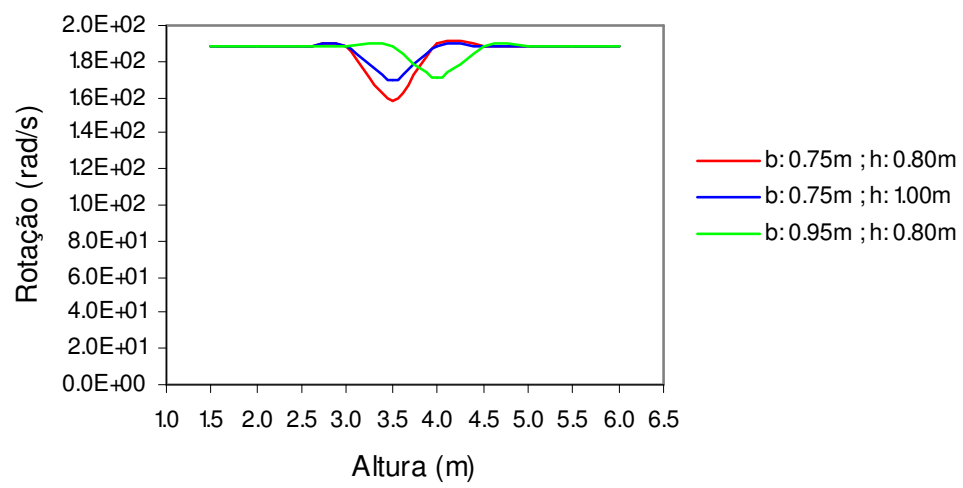

Gráfico 6.81 - Rotação $\omega 1 \mathrm{~m} \times$ Altura (r1.w1m: 2000mm/s)

Rotação $\omega 2 \mathrm{~m} \times$ Altura

r2. $22 \mathrm{~m}: 4000 \mathrm{~mm} / \mathrm{s}$ (r2: $21.34 \mathrm{~mm})$

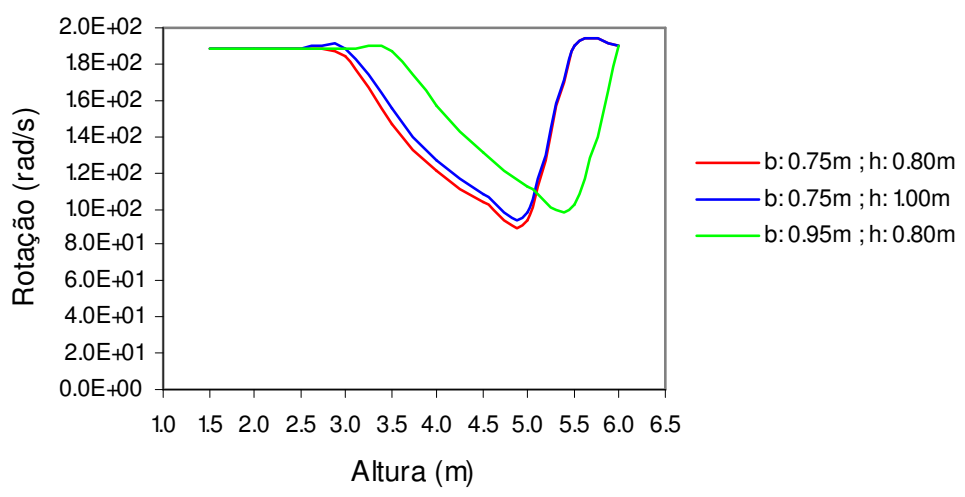

Gráfico 6.82 - Rotação w2m x Altura (r2.w2m: 4000mm/s) 
Deslocamento v0 x Altura

ro. $\omega 0 \mathrm{~m}: 6.3 \mathrm{~mm} / \mathrm{s}$ (r0: $0.034 \mathrm{~mm})$

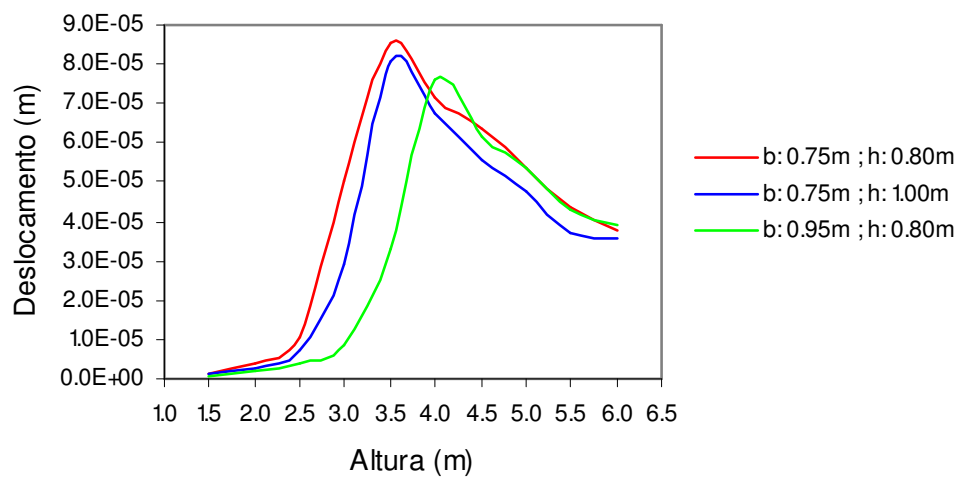

Gráfico 6.83 - Deslocamento v0 x Altura (r0.w0m: $6.3 \mathrm{~mm} / \mathrm{s}$ )

r1. $\omega 1 \mathrm{~m}: 2000 \mathrm{~mm} / \mathrm{s}$ (r1: $10.67 \mathrm{~mm}$ )

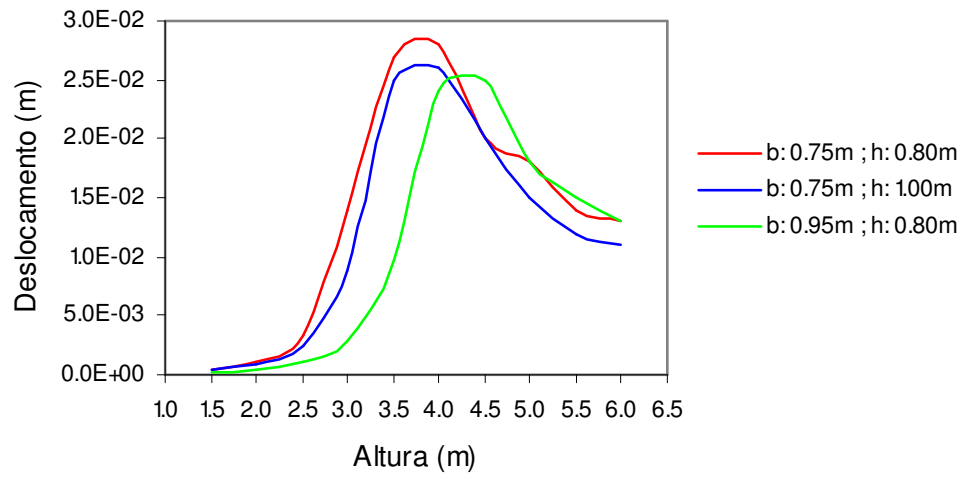

Gráfico 6.84 - Deslocamento v1 x Altura (r1.w1m: 2000mm/s)

Deslocamento v2 $x$ Altura

r2. $22 \mathrm{~m}: 4000 \mathrm{~mm} / \mathrm{s}$ (r2: $21.34 \mathrm{~mm})$

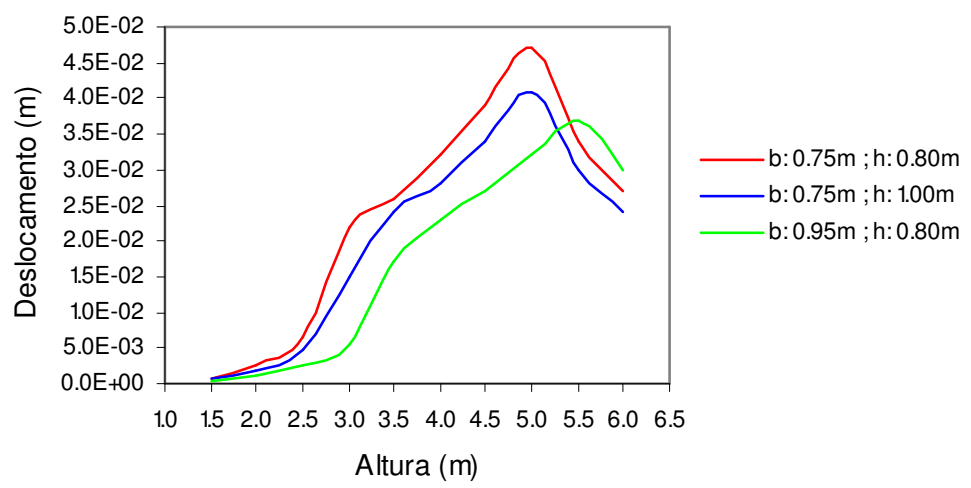

Gráfico 6.85 - Deslocamento v2 x Altura (r2.w2m: 4000mm/s) 


\section{DISCUSSÃO DOS RESULTADOS}

A aplicação da teoria de sistemas não-ideais realizada seguindo as prescrições da Norma ISO $1940 / 1$, referente à qualidade de balanceamento $(6,3 \mathrm{~mm} / \mathrm{s}$ para ventiladores), no sistema formado por motor elétrico e estrutura de concreto com dimensões iniciais a $0.75 \mathrm{~m}$ de largura, $0.80 \mathrm{~m}$ de comprimento e $2.00 \mathrm{~m}$ de altura, mostrou que o sistema se comporta como se fosse ideal. A rotação do motor é constante e igual à nominal, de $187.45 \mathrm{rad} / \mathrm{s}$ (gráfico 6.3), e tanto o sistema ideal como o não-ideal apresentam a mesma magnitude de deslocamento e velocidade (gráficos 6.1 e 6.2), ocorrendo pequenas variações no início do movimento devido ao transiente, mas com manutenção da estabilidade dos movimentos (gráfico 6.4).

Com relação à análise deste mesmo sistema para outras alturas da estrutura de concreto, os resultados revelaram que o seu comportamento continua sendo semelhante ao de um sistema ideal, ou seja, a rotação do motor é sempre igual a nominal (gráficos 6.8). Tal fato se repetiu para os outros dois sistemas estudados, que tiveram suas seções acrescidas de $0.20 \mathrm{~m}$, primeiramente na direção de maior inércia e, em segundo, na direção de menor inércia (gráficos 6.32 e 6.56, respectivamente).

Entretanto, quando adotada qualidade de balanceamento igual a $2000 \mathrm{~mm} / \mathrm{s}$ ou $4000 \mathrm{~mm} / \mathrm{s}$, não se respeitando o limite estabelecido pela Norma ISO 1940/1, que correspondente a $6,3 \mathrm{~mm} / \mathrm{s}$ para ventiladores, os três sistemas analisados passaram a apresentar comportamento não-ideal, em que há variação na rotação do motor para determinadas faixas de altura da estrutura de concreto. Esse comportamento pode ser visto nos mesmos gráficos anteriormente mencionados.

Interessante observar que a máxima energia consumida pela estrutura de concreto, tanto para sistema ideal como não-ideal, ocorre sempre nos estados ressonantes, situação em que a freqüência de operação do motor $187.45 \mathrm{rad} / \mathrm{s}$ se aproxima da freqüência natural da estrutura. Para o sistema analisado de dimensões iniciais, isso 
corresponde à estrutura com 3.35m de altura (gráfico 6.6); para os outros dois sistemas, as alturas são 3.40m e 3.80m (gráficos 6.30 e 6.54, respectivamente).

Quanto ao deslocamento da estrutura, no caso em que se trata de sistema ideal, assumiu-se teoricamente que os máximos deslocamentos ocorrem nos pontos (alturas) em que há maior consumo de energia, que, como exposto no parágrafo anterior, se dá no estado ressonante. Por outro lado, quando o sistema deixa de ser ideal, verifica-se um outro comportamento. Submetendo-se o sistema à qualidade de balanceamento igual a $2000 \mathrm{~mm} / \mathrm{s}$ ou $4000 \mathrm{~mm} / \mathrm{s}$, em ambos os casos se constatou que os maiores deslocamentos transversais da estrutura (gráfico 6.7) não ocorreram para a altura correspondente ao maior consumo de energia (gráfico 6.5) e sim para a altura correspondente à menor freqüência de operação do motor (gráfico 6.8). Contudo, há manutenção da estabilidade dos movimentos (gráficos 6.9 a 6.28). 0 mesmo comportamento se deu para os outros dois sistemas estudados. Pode-se dizer que as causas dessa diferença relacionam-se às características de linearidade versus não-linearidade dos sistemas.

Analisando-se os gráficos 6.77, 6.78 e 6.79, verificou-se ainda que a qualidade de balanceamento é responsável pela magnitude da energia consumida pela estrutura e conseqüentemente pelo comportamento do sistema, gráficos 6.80 a 6.85. Pode-se, inferir que um aumento de $0.20 \mathrm{~m}$ no comprimento da base, no sentido de maior inércia, não acarreta mudanças significativas no movimento do sistema, se comparado ao inicial. No entanto, o mesmo acréscimo, no sentido de menor inércia, ocasiona mudanças expressivas; porque há um distanciamento maior da freqüência natural deste sistema em relação ao inicial.

Com relação à rigidez geométrica, pôde-se ainda constatar (gráfico 7.1) que ela em nada afetou o sistema em análise $(b=0.75 \mathrm{~m}, \mathrm{~h}=0.80 \mathrm{~m})$, pois enquanto sua ordem de grandeza é de $10^{3} \mathrm{~N} / \mathrm{m}$, a da rigidez à flexão é de $10^{8} \mathrm{~N} / \mathrm{m}$. 


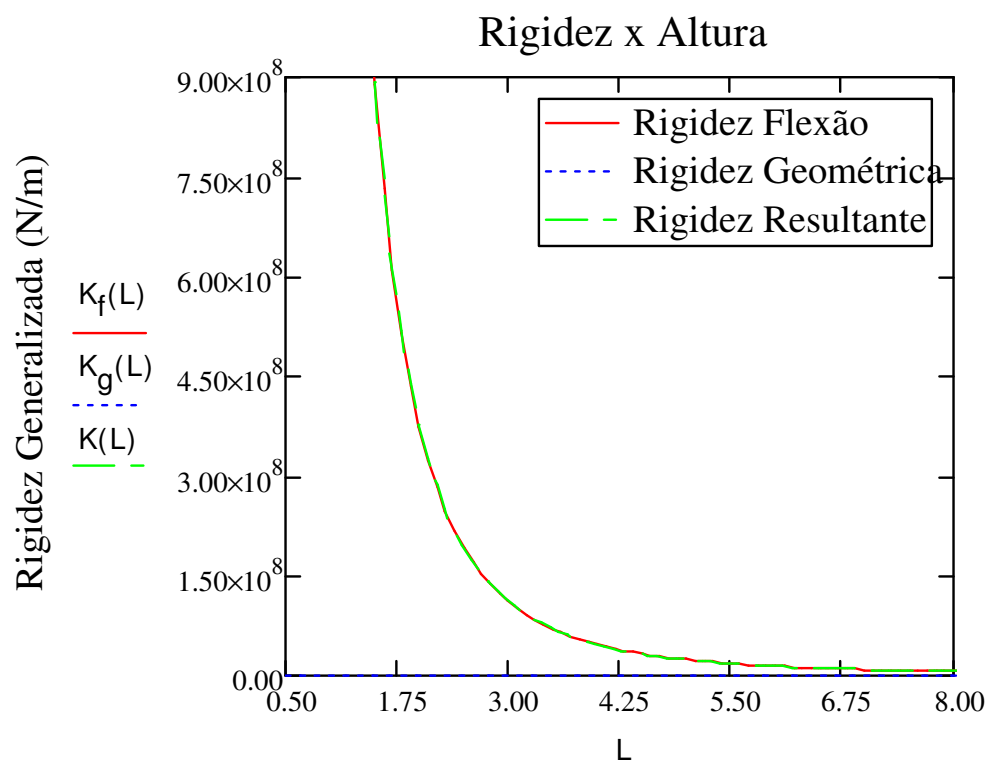

Altura (m)

Gráfico 7.1 - Comparação entre rigidez à flexão e geométrica x Altura

Para que o efeito da rigidez geométrica começasse a influenciar o sistema (gráfico 7.2), a massa do equipamento, no caso o motor elétrico de $1.455 \mathrm{~kg}$, deveria ser de pelo menos 10 mil ton, ou seja, aproximadamente 6.875 vezes maior.

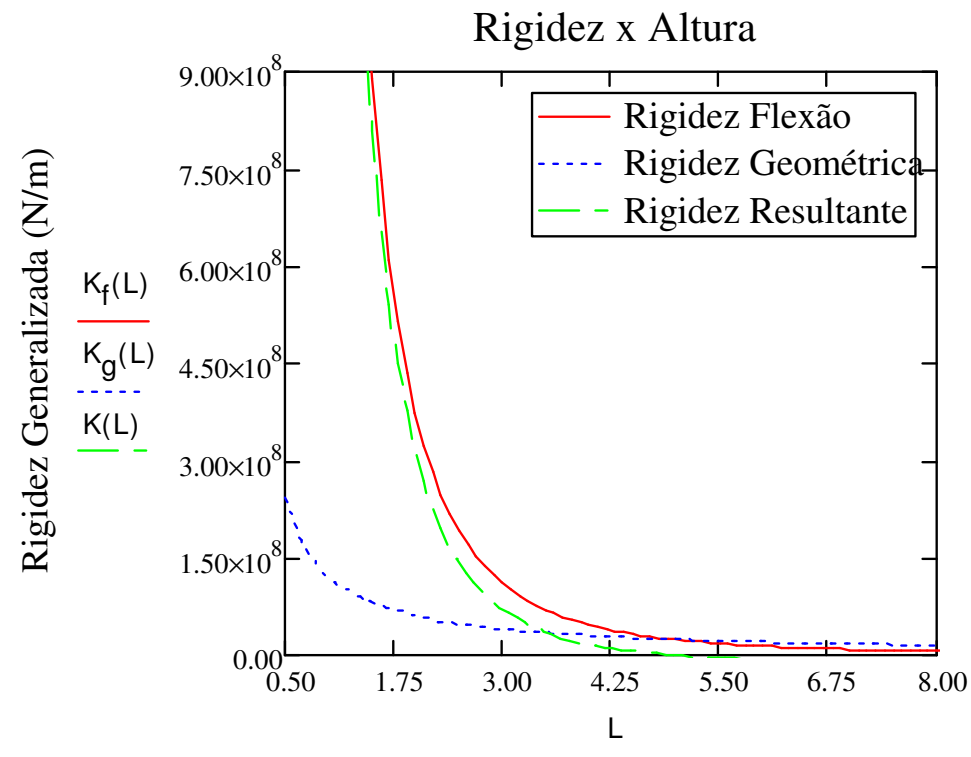

Altura (m)

Gráfico 7.2 - Influência da rigidez geométrica x Altura 


\section{CONCLUSÕES}

Quando da realização de projetos estruturais, raramente é considerada a interação entre o motor e sua estrutura dentro da perspectiva de um funcionamento não-ideal. Parte-se da premissa de que o comportamento do sistema será ideal, por vários motivos, tais como falta de conhecimento do assunto em pauta, dificuldade em se obter informações técnicas específicas sobre os equipamentos (os dados fornecidos pelos fabricantes de equipamentos rotativos normalmente contemplam somente informações básicas, do tipo peso, esforço estaticamente equivalente ao dinâmico e freqüência de rotação da máquina), como também dificuldades de manipulação de complexas equações para obtenção de resultados. Tanto é, que, segundo BALTHAZAR, 2004, p. 237, "The behavior of ideal vibrating systems is well known in the current literature, but there are few results on non-ideal ones".

Sérias conseqüências podem ocorrer se um sistema dimensionado de maneira ideal passar a funcionar como não-ideal. O sistema pode não atingir a performance para qual o foi projetado, gerando perda de eficiência e rendimento, provável redução da vida útil dos equipamentos e elevados prejuízos financeiros. Interessante, ainda, enfatizar que a as empresas fornecedoras de motores deixam ao engenheiro civil a responsabilidade pelo dimensionamento da estrutura que dará suporte à máquina, esperando que esta funcione de acordo com as especificações mecânicas de projeto.

Diante do exposto, o objetivo desse trabalho foi mensurar até que ponto a premissa de que não há problemas em se projetar uma estrutura assumindo que ela faz parte de um sistema ideal corresponde à realidade. Para isso, partiu-se da hipótese de comportamento não-ideal do sistema e verificaram-se as amplitudes de deslocamento, bem como a variação da freqüência de rotação do motor, visando-se contribuir para o estudo dos sistemas não-ideais. 
Frente às exigências dos próprios equipamentos, a Norma ISO 1940/1 estabelece estreitos limites para qualidade de balanceamento. No caso de ventiladores industriais, esse limite é de $r \cdot \omega_{m}=6,3 \mathrm{~mm} / \mathrm{s}$, que corresponde a uma excentricidade máxima permitida de $0.034 \mathrm{~mm}$ para a massa girante de um motor elétrico com rotação de $187.45 \mathrm{rad} / \mathrm{s}$. O limite é tão severo para o equipamento que a energia consumida pela estrutura, decorrente de seu acoplamento dinâmico com o motor, é mínima.

Outro fato constatado é que os motores com aplicações industriais normalmente apresentam curvas de conjugado característico líquido $L_{(\dot{\varphi})}-H_{(\dot{\varphi})}$, muito acima da curva de energia consumida pela estrutura. Nos sistemas analisados a ordem de grandeza da curva do motor é de $10^{3} \mathrm{Nm}$ e das estruturas, de $10^{-2} \mathrm{Nm}$. Desta forma, em virtude do rigoroso limite estabelecido pela Norma ISO 1940/1 e das diferenças de ordem de grandeza da curva de conjugado e de energia, os sistemas estudados não apresentaram ocorrência do Efeito Sommerfeld ou "fenômeno do salto".

Conclui-se, portanto, que, para as estruturas em questão, se a Norma for seguida, a premissa de funcionamento do sistema como ideal é valida. Por outro lado, quando os limites da Norma não são respeitados, como no caso da imposição ao sistema de qualidades de balanceamento de $2000 \mathrm{~mm} / \mathrm{s}$ e $4000 \mathrm{~mm} / \mathrm{s}$, que correspondem a excentricidades de $10.67 \mathrm{~mm}$ e $21.34 \mathrm{~mm}$, respectivamente, para uma velocidade de rotação nominal de $187.45 \mathrm{rad} / \mathrm{s}$, há ocorrência do fenômeno. Esses valores de excentricidade, embora impraticáveis para motores, são possíveis em projetos de estruturas, como por exemplo em um pilar, cuja excentricidade de projeto é a soma das excentricidades de carga, construtiva e de segunda ordem.

Outro ponto a se concluir refere-se às dimensões da estrutura, no caso de situações passiveis de ocorrência do Efeito Sommerfeld. Nestas situações, verificou-se que um aumento nas dimensões da estrutura na direção do movimento do sistema (menor inércia) seria mais recomendável do que o aumento da dimensão no sentido perpendicular ao movimento, o de maior inércia da estrutura. 
A respeito da rigidez geométrica, pode-se ainda inferir que ela em nada afetou o sistema em análise $(b=0.75 \mathrm{~m}, \mathrm{~h}=0.80 \mathrm{~m})$, pois enquanto sua ordem de grandeza é de $10^{3} \mathrm{~N} / \mathrm{m}$, a da rigidez à flexão é de $10^{8} \mathrm{~N} / \mathrm{m}$. Para que seu efeito começasse a influenciar o sistema, a massa do equipamento, no caso o motor elétrico de $1.455 \mathrm{~kg}$, deveria ser de aproximadamente 6.875 vezes maior.

Finalmente, como extensão desse trabalho, propõe-se, em estudos futuros, com base na Teoria de Sistemas Ideais e Não-Ideais, a análise de sistemas sobre apoios elásticos, de modo a simular a interação entre a estrutura e o solo. 


\section{REFERÊNCIAS}

BALTHAZAR, J. M.; CHESHANKOV, B. I.; RUSCHEV, D. T.; BARBANTI, L.; WEBER, $\mathrm{H}$. I. Remarks on the passage through resonance of a vibrating system with two degrees of freedom, excited by a non-ideal energy source. Journal of Sound and Vibration, London, v. 239, n. 5, p. 1075-1085, 2001.

BALTHAZAR, J. M.; MOOK, D. T.; WEBER, H. I.; BRASIL, R. M. L. R. F.; FENILI, A.; BELATO, D.; FELIX, J. L. P. An overview on non-ideal vibrations. Meccanica, Itália, v. 38, p. 613-621, 2003.

BALTHAZAR, J. M.; BRASIL, R. M. L. R. F.; GARZERI, F. J. On non-ideal simple portal frame structural model: experimental results under non-ideal excitation. Applied Mechanics and Materials, Grã-Bretanha, v. 1, n. 2, p. 51-58, 2004.

BALTHAZAR, J. M.; BRASIL, R. M. L. R. F.; WEBER, H. I.; FENILI, A.; BELATO, D.; FELIX, J. L. P.; GARZERI, F. J. A review of new vibration issues due to non-ideal energy sources. In: UDAWADIA, F. E.; WEBER, H. I.; LEITMANN, G. (Org.) Dynamics systems and control. London: Taylor \& Francis, 2004. p. 237-258.

BRASIL, R. M. L. R. F.; MOOK, D. T. Vibration of portal frame excited by a non ideal motor. In: CONFERENCE ON NON LINEAR VIBRATIONS, STABILITY AND DYNAMICS OF STRUCTURES, 5., 1994, Blacksburg. Abstracts. Blacksburg: Virginia Polytechnic Institute and State University, 1994. p. 249-249.

BRASIL, R. M. L. R. F.; BALTHAZAR, J. M. Nonlinear oscillations of a portal frame structure excited by a nonideal motor. In: INTERNATIONAL CONFERENCE ON CONTROL OF OSCILLATIONS AND CHAOS, 2., 2000, Petersburg, Rússia. Proceedings... St. Petersburg, Rússia: IEEE, 2000. p. 275-285.

BRASIL, R. M. L. R. F. Não-linearidade geométrica na dinâmica de estruturas aporticadas planas: um tratamento pelo método dos elementos finitos. 1990. $211 \mathrm{p}$. Tese (Doutorado) - Escola Politécnica, Universidade de São Paulo, São Paulo, 1990.

CLOUGH, R.W.; PENZIEN, J. Dynamics of structures. $2^{\text {nd }}$ ed. New York: McGrawHill, 1994. 738 p.

COSTA, M. N. da; GALHA NITZ, R. Mathcad 12: guia prático. São Paulo: Érica, 2005. $248 \mathrm{p}$. 
FEITOSA, L.C.S. Controle por impacto de vibrações estruturais excitadas por carregamentos não-ideais. 2006. 117 p. Dissertação (Mestrado) - Escola Politécnica, Universidade de São Paulo, São Paulo, 2006.

FELIX, J. L. P. Teoria de sistemas vibratórios aporticados não-lineares e nãoideais. 2002. 181 p. Tese (Doutorado) - Universidade Estadual de Campinas, Campinas, 2002.

FELIX, J. L. P; BALTHAZAR, J. M.; BRASIL, R. M. L. R. F. On tuned liquid column dampers mounted on a structural frame under a non-ideal excitation. Journal of Sound and Vibration, London, v. 282, p. 1285-1292, 2005b.

GARZERI, F. J. Dinâmica não linear de um pórtico plano sob carregamento não ideal: análise numérica e experimental. 2001. 80 p. Tese (Doutorado) - Escola Politécnica, Universidade de São Paulo, São Paulo, 2001.

INTERNATIONAL ORGANIZATION FOR STANDARDIZATION. ISO 1940-1: mechanical vibration - balance quality requirements for rotors in a constant state part 1: specification and verification of balance tolerances. Geneve, 2003. 28 p.

KONONENKO, V. O. Vibrating Systems with Limited Power Supply. London: Iliffe Books, 1969. $236 \mathrm{p}$.

KUROIWA, T. Controle passivo de vibrações de bases de motores não-ideais. 2003. 107 p. Dissertação (Mestrado) - Escola Politécnica, Universidade de São Paulo, São Paulo, 2003.

NAYFEH, A. H.; MOOK, D. T. Nonlinear oscillations. New York: John Willey, 1979. $704 \mathrm{p}$.

NÓBREGA, P. G. B. Auto-sincronização de motores não-ideais apoiados em estruturas elásticas. 1994. 109 p. Dissertação (Mestrado) - Escola Politécnica, Universidade de São Paulo, São Paulo, 1994.

TSUCHIDA, M.; GUILHERME, K. L.; BALTHAZAR, J. M. On chaotic vibrations of a non-ideal system with two degrees of freedom: 1:2 resonance and Sommerfeld effect. Journal of Sound and Vibration, London, v. 282, p. 1201-1207, 2005.

WEG EQUIPAMENTOS ELÉTRICOS SA. Motores elétricos. Jaraguá do Sul, SC, 2005. 147 p. 


\section{REFERÊNCIAS COMPLEMENTARES}

ALMEIDA, E. S. N. Introdução à análise dinâmica de fundações de máquinas. 1989. 120 p. Dissertação (Mestrado) - Escola Politécnica, Universidade de São Paulo, São Paulo,1989.

BRASIL, R. M. L. R. F.; BALTHAZAR J. M. Chaotic motions of a simple portal frame. In: CONGRESSO NACIONAL DE ENGENHARIA MECÂNICA, 2000, Natal. CONEM 2000: anais. Caucaia: Nordeste Digital Line, 2000. 1 CD-ROM.

CRANDALL, S. H. Engineering analysis: a survey of numerical procedures. New York: McGraw-Hill, 1956. 417 p.

FALCONE, A. G. Eletromecânica: máquinas elétricas rotativas. São Paulo: Edgard Blücher, 1979. v. 2.

FELIX, J. L. P; BALTHAZAR, J. M.; BRASIL, R. M. L. R. F. On saturation control of a non-ideal vibrating portal frame foundation type shear-building. Journal of Vibration and Control, London, v. 11, p. 121-136, 2005a.

LOBOSCO, O. S. ; DIAS, J. L. P. C. Seleção e aplicação de motores elétricos. São Paulo, McGraw-Hill: Siemens, 1988. v. 1. 34 p.

MAZZILI, C. E. N.; SOARES, M. E. S. Análise dinâmica de estruturas com comportamento linear. São Paulo: EPUSP, 2006. Notas de aula da disciplina PEF5735, ministrada no curso de pós-graduação de engenharia civil.

SOUZA, S. L. T.; CALDAS, I. L.; VIANA, R. I.; BALTHAZAR, J. M.; BRASIL, R. M. L. R. F. Impact dampers for controlling chaos in systems with limited power supply. Journal of Sound and Vibration, London, v. 279, p. 955-967, 2005. 


\section{APÊNDICE A - ROTINA PARA REGRESSÃO POLINOMIAL}

Parte I - Entrada de Dados

Dados $:=\left(\begin{array}{cc}0.00 & 2587.20 \\ 9.42 & 2528.40 \\ 18.85 & 2469.60 \\ 28.27 & 2434.32 \\ 37.70 & 2399.04 \\ 47.12 & 2363.76 \\ 56.55 & 2328.48 \\ 65.97 & 2293.20 \\ 75.40 & 2257.92 \\ 84.82 & 2222.64 \\ 94.25 & 2199.12 \\ 103.67 & 2187.36 \\ 113.10 & 2175.60 \\ 131.95 & 2163.84 \\ 141.37 & 2140.03 \\ 150.80 & 2199.12 \\ 160.22 & 2257.92 \\ 169.65 & 2469.60 \\ 179.07 & 2704.80 \\ 180.96 & 2528.40 \\ 182.84 & 2234.40 \\ 184.73 & 1764.00 \\ 186.61 & 1176.00 \\ 188.50 & 0.00\end{array}\right)$

Variável independente:

$x:=$ Dados $^{\langle 0\rangle}$

(rotação do motor em rad/s)

Variável dependente:

$y:=\operatorname{Dados}^{\langle 1\rangle}$

(conjugado do motor em Nm)

Grau do polinômio ajustado:

$\mathrm{k}:=12$

Coeficientes do polinômio ajustado:

coef $:=\operatorname{regress}(\mathrm{x}, \mathrm{y}, \mathrm{k})$

Polinômio ajustado:

$\operatorname{CCL}\left(\omega_{\mathrm{m}}\right):=\operatorname{interp}\left(\operatorname{coef}, \mathrm{x}, \mathrm{y}, \omega_{\mathrm{m}}\right)$ 
Gráfico A.1 - Polinômio $1^{\circ} \mathrm{grau}$

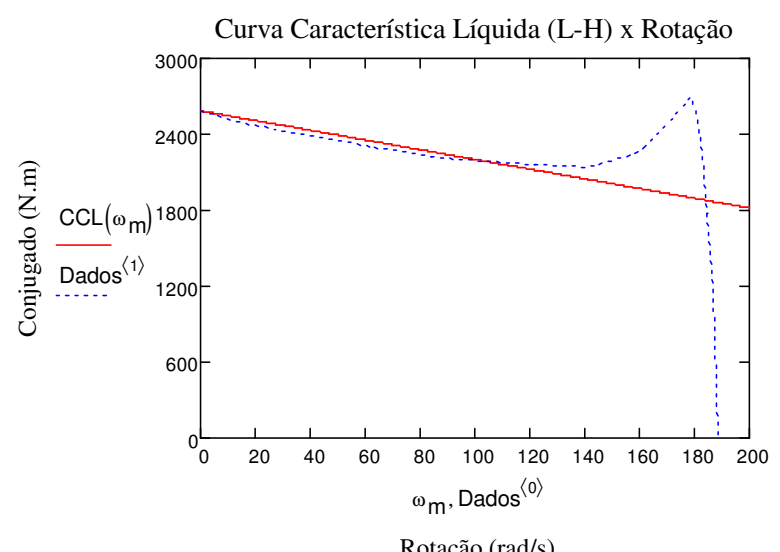

Gráfico A.3 - Polinômio $3^{\circ}$ grau

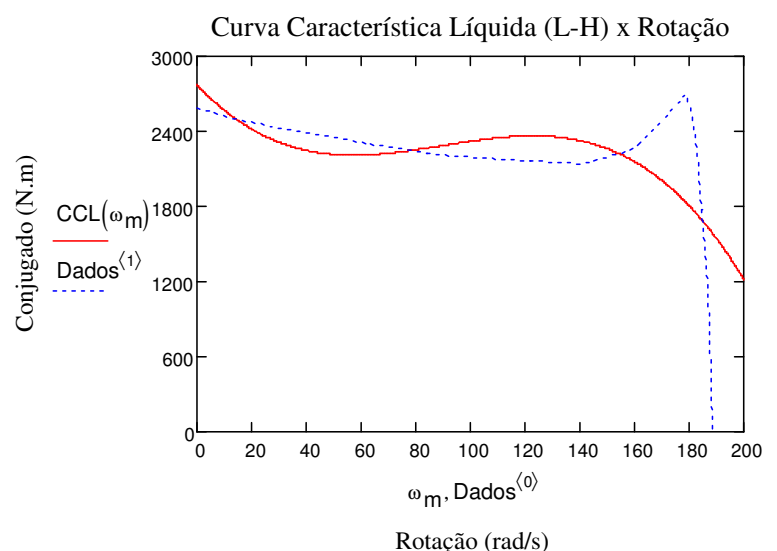

Gráfico A.5 - Polinômio $5^{\circ}$ grau

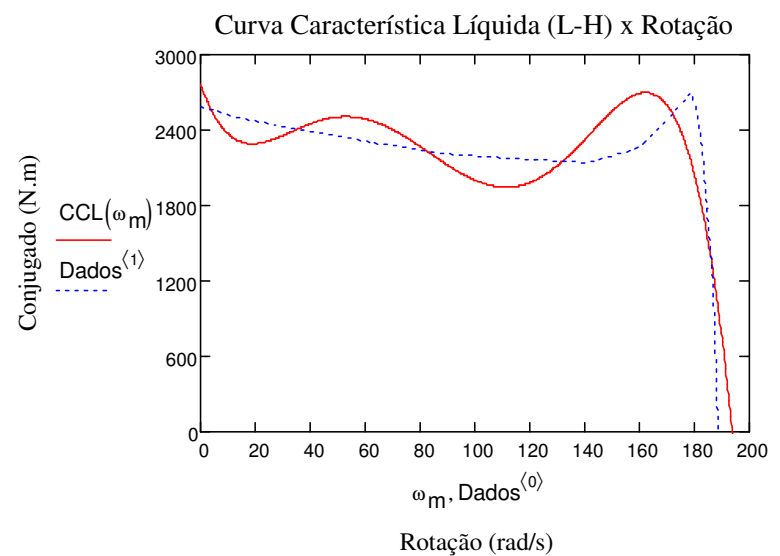

Gráfico A.2 - Polinômio $2^{\circ}$ grau

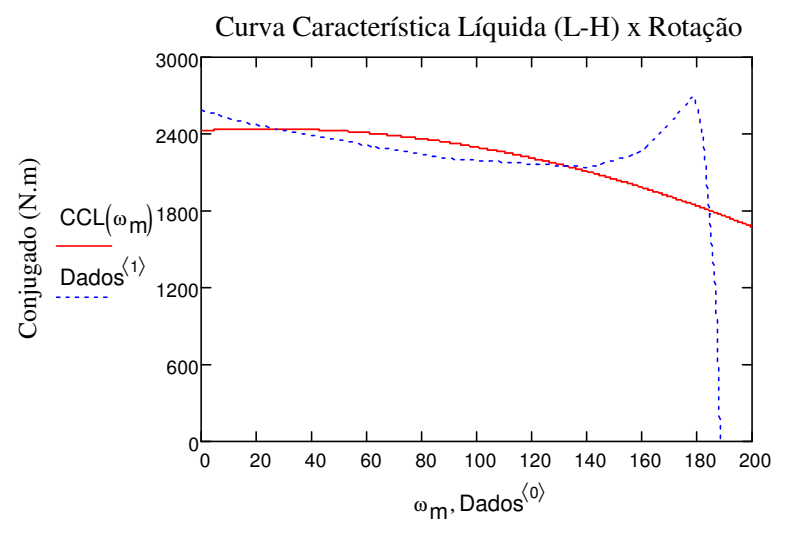

Rotação (rad/s)

Gráfico A.4 - Polinômio $4^{\circ}$ grau

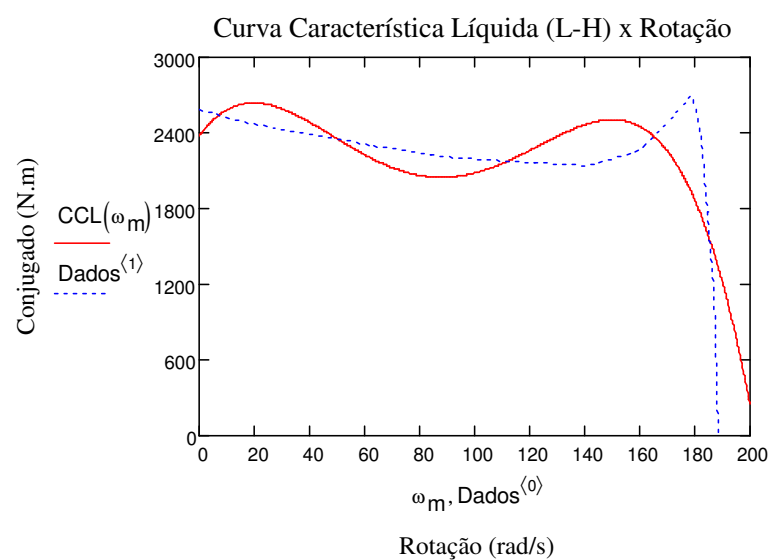

Gráfico A.6 - Polinômio $6^{\circ}$ grau

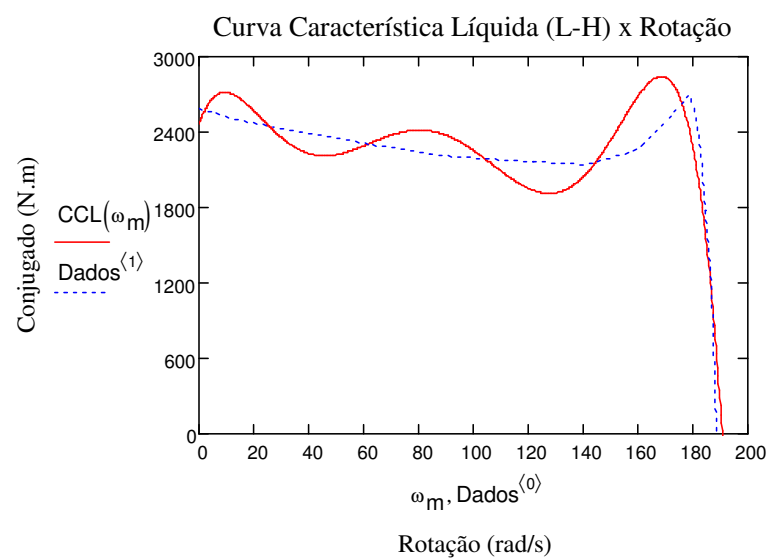


Gráfico A.7 - Polinômio $7^{\circ}$ grau

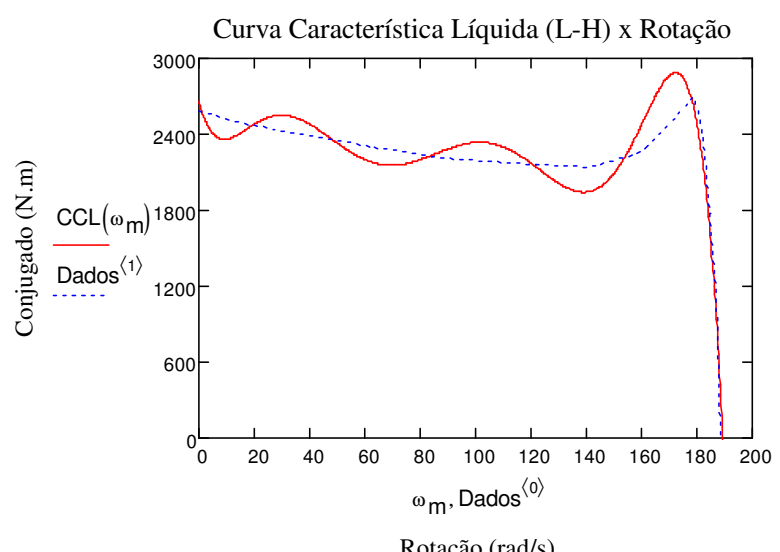

Gráfico A.9 - Polinômio $9^{\circ}$ grau

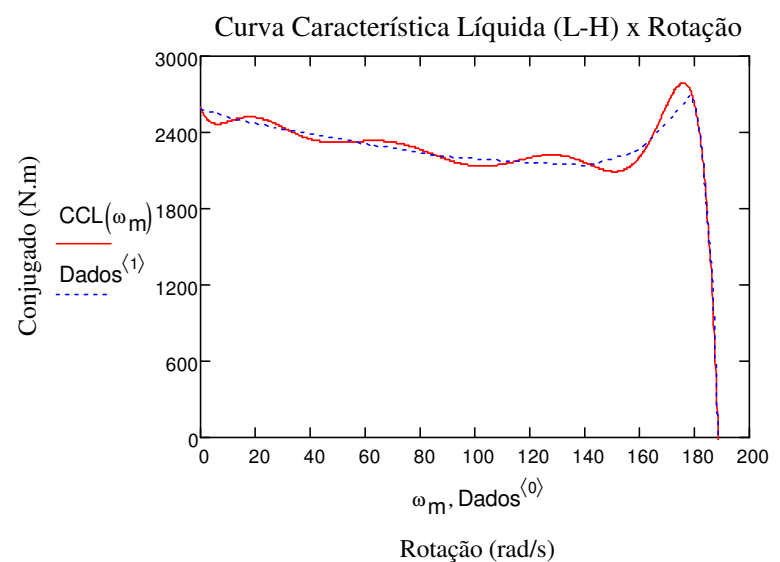

Gráfico A.11 - Polinômio $11^{\circ}$ grau

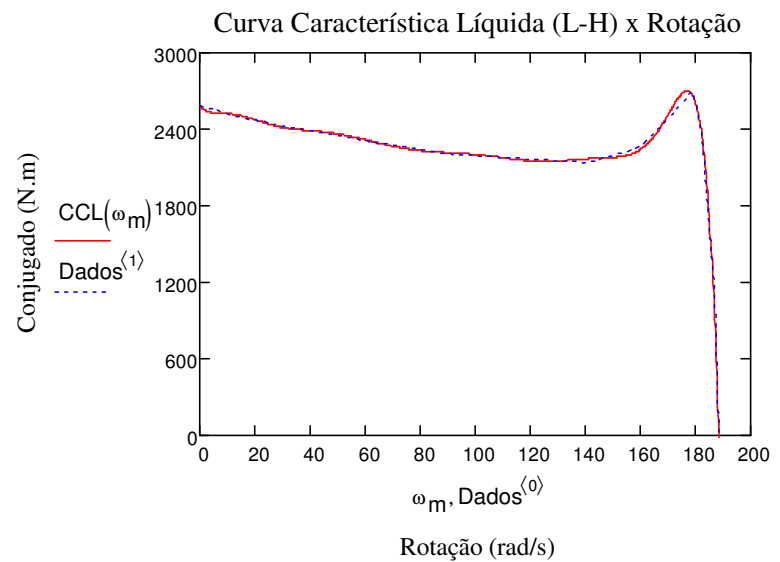

Gráfico A.8 - Polinômio $8^{\circ}$ grau

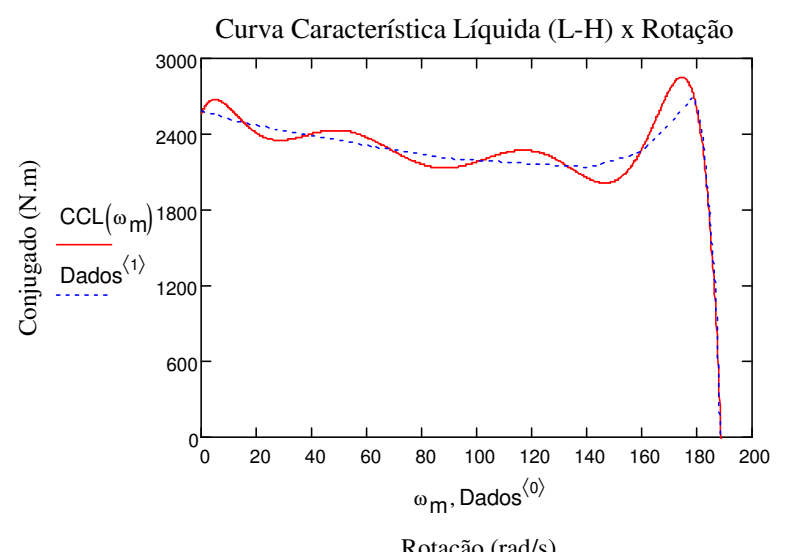

Gráfico A.10 - Polinômio $10^{\circ} \mathrm{grau}$

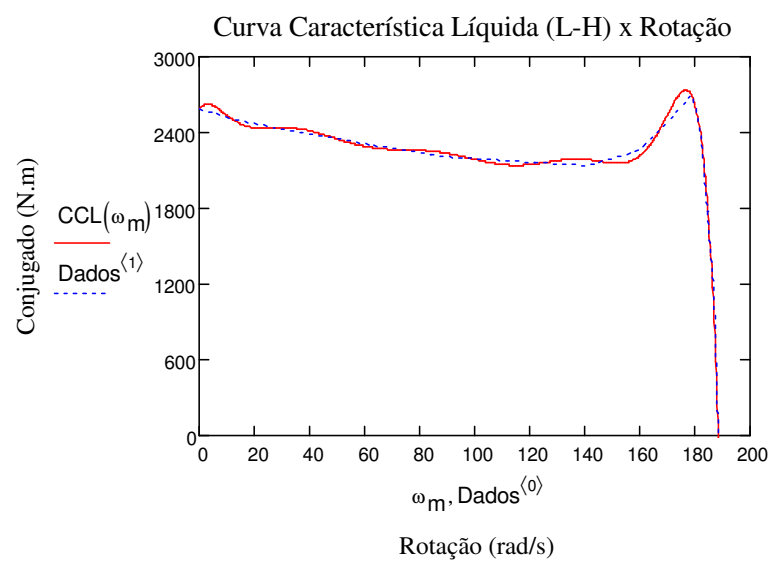

Gráfico A.12 - Polinômio $12^{\circ}$ grau

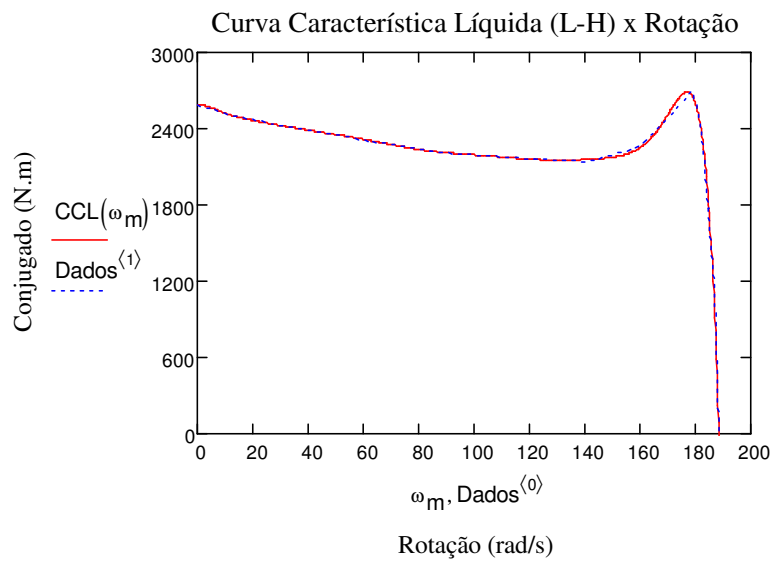


Gráfico A.13 - Polinômio $13^{\circ}$ grau

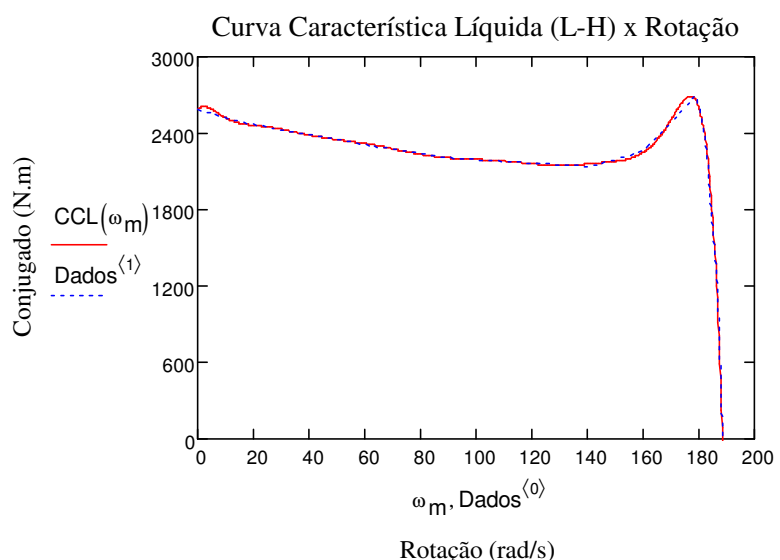

Gráfico A.15 - Polinômio 15º grau

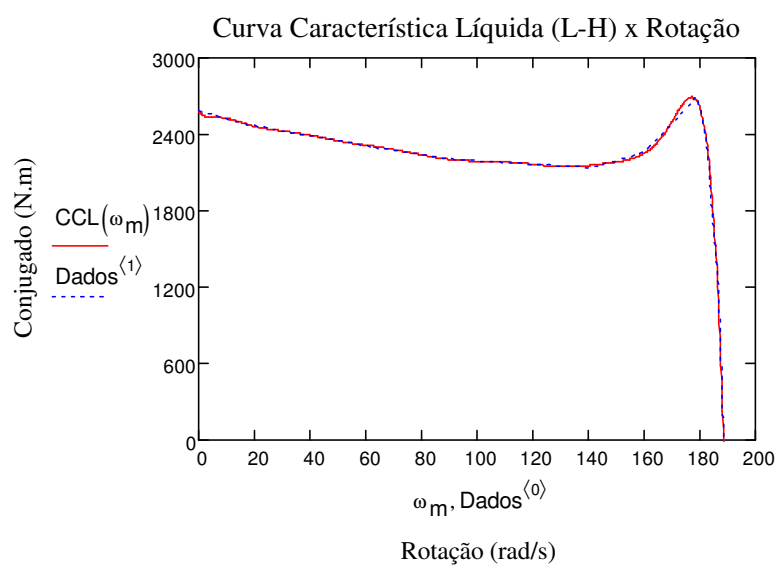

Gráfico A.17 - Polinômio $17^{\circ}$ grau

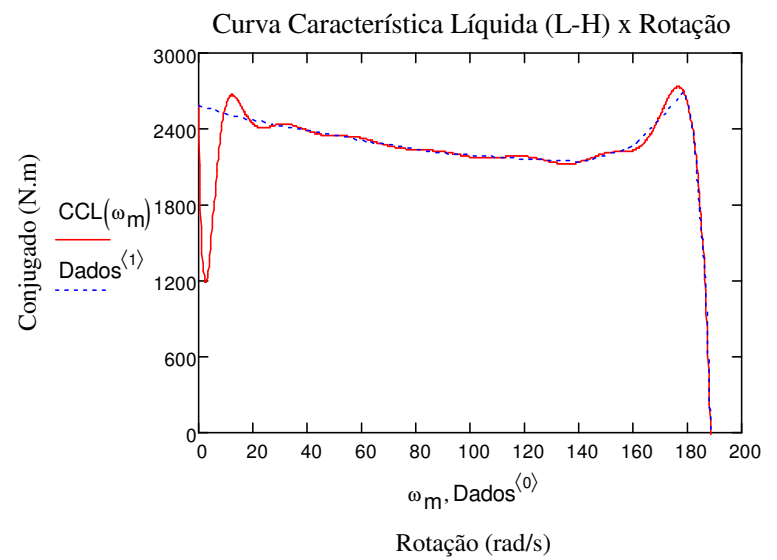

Gráfico A.14 - Polinômio $14^{\circ}$ grau

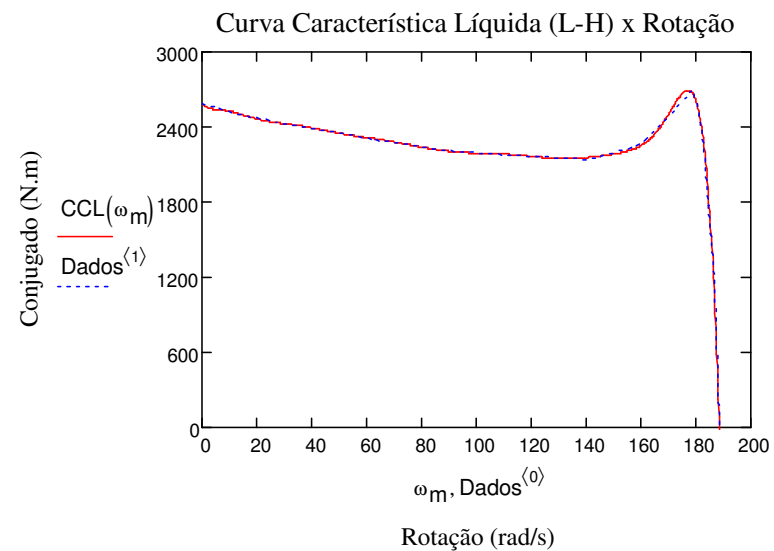

Gráfico A.16 - Polinômio $16^{\circ}$ grau

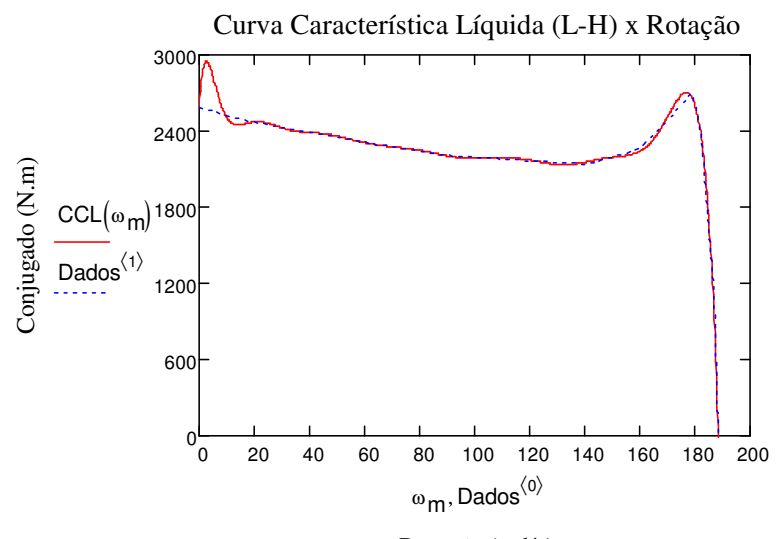

Rotação (rad/s)

\section{Gráfico A.18 - Polinômio $18^{\circ}$ grau}

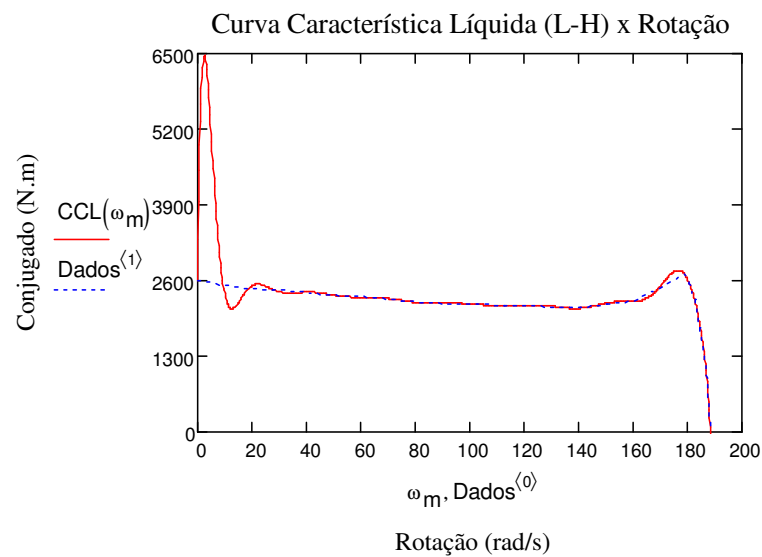


Parte I - Entrada de Dados

a) Qualidade de Balanceamento Conforme Norma ISO 1940/1

ventilador industrial :

$$
\begin{aligned}
& \mathrm{v}:=6.3 \frac{\mathrm{mm}}{\mathrm{s}} \\
& \mathrm{g}=9.81 \frac{\mathrm{m}}{\mathrm{s}^{2}}
\end{aligned}
$$

aceleração da gravidade :

b) Características do Motor WEG - Tríasico de Indıção - Rotor Gaiola

massa total :

massa girante (rotor) :

peso total :

rotação nominal :

excentricidade da massa girante :

c) Características da Estrutura

Material

peso específico do concreto :

$$
\gamma_{\mathrm{C}}:=2.5 \cdot 10^{4} \frac{\mathrm{N}}{\mathrm{m}^{3}}
$$

módulo de elasticidade do concreto :

$$
E:=3.5 \cdot 10^{10} \frac{\mathrm{N}}{\mathrm{m}^{2}}
$$

Geometria

dimensão menor da base :

dimensão maior da base :

altura :

$$
\mathrm{m}_{\mathrm{m}}:=1455 \mathrm{~kg}
$$

$$
\mathrm{m}_{\mathrm{g}}:=0.65 \cdot \mathrm{m}_{\mathrm{m}} \quad \mathrm{m}_{\mathrm{g}}=946 \mathrm{~kg}
$$

$$
P:=m_{m} \cdot g
$$

$P=14269 N$

$$
\begin{array}{ll}
\omega_{\mathrm{pm}}:=1790 \mathrm{ppm} & \omega_{\mathrm{pm}}=187.45 \cdot \frac{\mathrm{rad}}{\mathrm{s}} \\
\mathrm{e}:=\frac{\mathrm{v}}{\omega_{\mathrm{pm}}} & \mathrm{e}=3.36 \times 10^{-5} \mathrm{~m}
\end{array}
$$


Cálculo

inércia em relação ao eixo y :

$\mathrm{I}:=\frac{\mathrm{h} \cdot \mathrm{b}^{3}}{12}$

$I=2.81 \times 10^{-2} \cdot \mathrm{m}^{4}$

massa linear :

$\mathrm{m}_{\mathrm{l}}:=\frac{\mathrm{h} \cdot \mathrm{b} \cdot \gamma_{\mathrm{c}}}{\mathrm{g}} \quad \mathrm{m}_{\mathrm{l}}=1.53 \times 10^{3} \frac{\mathrm{kg}}{\mathrm{m}}$

massa generalizada :

$\mathrm{m}_{\mathrm{e}}:=\left(\frac{3}{2}-\frac{4}{\pi}\right) \cdot \mathrm{m}_{\mathrm{l}} \cdot \mathrm{L} \quad \mathrm{m}_{\mathrm{e}}=693.69 \mathrm{~kg}$

rigidez a flexão generalizada :

$K_{f}:=\frac{\pi^{4} \cdot E \cdot I}{32 \cdot L^{3}} \quad K_{f}=3.75 \times 10^{8} \cdot \frac{N}{m}$

rigidez geométrica generalizada :

$\mathrm{K}_{\mathrm{g}}:=\frac{\pi^{2} \cdot \mathrm{P}}{8 \cdot \mathrm{L}}$

$\mathrm{K}_{\mathrm{g}}=8.80 \times 10^{3} \cdot \frac{\mathrm{N}}{\mathrm{m}}$

rigidez generalizada :

$\mathrm{K}:=\mathrm{K}_{\mathrm{f}}-\mathrm{K}_{\mathrm{g}}$

$\mathrm{K}=3.75 \times 10^{8} \cdot \frac{\mathrm{N}}{\mathrm{m}}$

d) Características do Sistema

massa total :

$M_{0}:=m_{m}+m_{e} \quad M_{0}=2.15 \times 10^{3} \mathrm{~kg}$

freqüência natural :

$\omega:=\sqrt{\frac{\mathrm{K}}{\mathrm{M}_{0}}} \quad \omega=417.51 \cdot \frac{\mathrm{rad}}{\mathrm{s}}$

$\mathrm{f}_{0}:=\frac{\omega}{2 \cdot \pi}$

$f_{0}=66.45 \frac{1}{s}$

taxa de amortecimento :

$\xi:=0.05$

amortecimento :

$C:=2 \cdot M_{0} \cdot \omega \cdot \xi$

$\mathrm{C}=8.97 \times 10^{4} \frac{\mathrm{kg}}{\mathrm{s}}$

excentricidade :

$r:=e$

$\mathrm{r}=3.36 \times 10^{-5} \mathrm{~m}$

massa desbalanceada :

$m_{d}:=\frac{m_{g} \cdot e}{r} \quad m_{d}=946 \mathrm{~kg}$ 
Parte II - Método Runge Kutta com Passo Fixo

a) Intervalo de Integrȩão

intervalo :

inicio $:=0$

fim $:=200$

número de partes por ciclo :

$p:=20 s$

número de passos :

npasso $:=\frac{\text { fim }- \text { inicio }}{\frac{1}{p} \cdot \frac{1}{f_{0}}}$

número de passos calculado :

npasso $=2.658 \times 10^{5}$

número de passos fixado :

npasso $:=260000$

b) Condições Iniciais

aceleração estrutura :

$v_{\mathrm{pp}}:=0$

velocidade estrutura :

$v_{p}:=0$

c) Vetor Condições Iniciais

$$
\text { Condini }_{i}:=\left(\begin{array}{c}
v_{p} \\
v_{p p}
\end{array}\right)
$$

d) Vetor Derivada $D(t, R)$

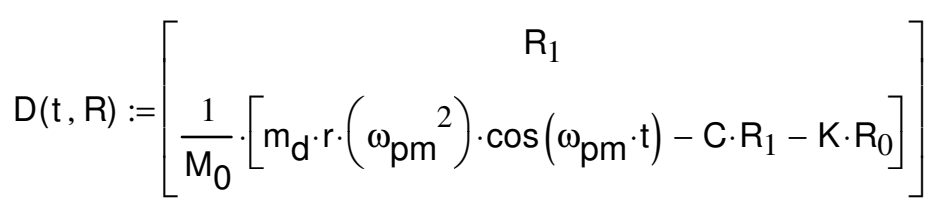


$S:=\operatorname{rkfixed}\left(\right.$ Cond $_{\text {ini }}$, inicio , fim , npasso, D)

$$
\mathrm{t}:=\mathrm{S}^{\langle 0\rangle} \quad \operatorname{des}_{\mathrm{si}}:=\mathrm{S}^{\langle 1\rangle} \quad \mathrm{vel}_{\mathrm{si}}:=\mathrm{S}^{\langle 2\rangle}
$$

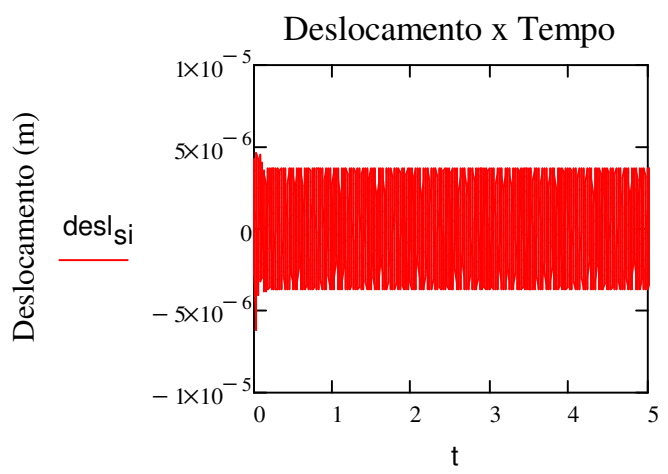

Tempo (s)

$\max \left(\right.$ desl $\left._{\mathrm{si}}\right)=4.587 \times 10^{-6}$

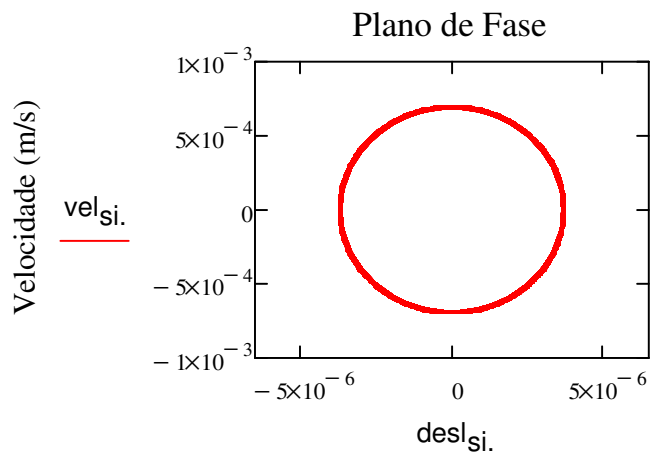

Deslocamento (m)

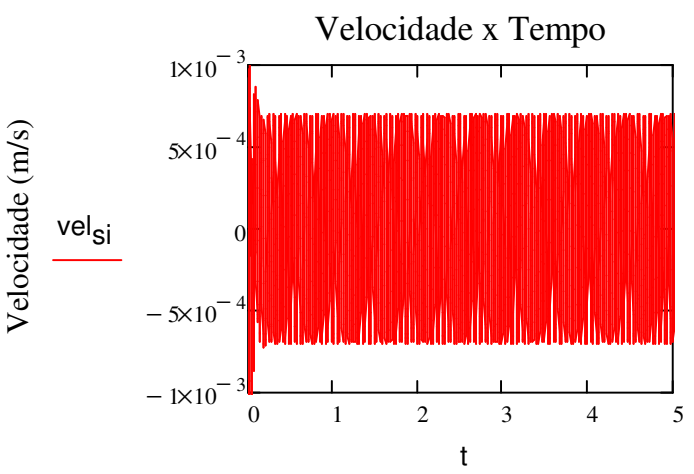

Tempo (s)

$\max \left(\operatorname{vel}_{\mathrm{si}}\right)=1.287 \times 10^{-3}$ 


\section{APÊNDICE C - ROTINA DE CÁLCULO SISTEMA NÃO-IDEAL - 2GL}

Parte I - Entrada de Dados

a) Qualidade de Balanceamento Conforme Norma ISO 1940/1

ventilador industrial :

$$
\begin{aligned}
& v:=6.3 \frac{\mathrm{mm}}{\mathrm{s}} \\
& \mathrm{g}=9.81 \frac{\mathrm{m}}{\mathrm{s}^{2}}
\end{aligned}
$$

aceleração da gravidade :

b) Características do Motor WEG - Tríasico de Indução - Rotor Gaiola

massa total :

massa girante (rotor) :

peso total :

rotação nominal :

excentricidade da massa girante :

inércia rotacional caracteństica :

c) Características da Estrutura

Material

peso específico do concreto :

$$
\gamma_{c}:=2.5 \cdot 10^{4} \frac{\mathrm{N}}{\mathrm{m}^{3}}
$$

módulo de elasticidade do concreto :

$$
E:=3.5 \cdot 10^{10} \frac{\mathrm{N}}{\mathrm{m}^{2}}
$$

Geometria

dimensão menor da base :

$\mathrm{b}:=0.75 \mathrm{~m}$

dimensão maior da base :

$\mathrm{h}:=0.80 \mathrm{~m}$

altura :

$$
m_{m}:=1455 \mathrm{~kg}
$$

$m_{g}:=0.65 \cdot m_{m}$

$m_{g}=946 \mathrm{~kg}$

$P=14269 N$$$
\omega_{\mathrm{pm}}=187.45 \cdot \frac{\mathrm{rad}}{\mathrm{s}}
$$$$
\mathrm{e}=3.36 \times 10^{-5} \mathrm{~m}
$$

$\mathrm{J}_{\mathrm{m}}:=6.34 \mathrm{~kg} \cdot \mathrm{m}^{2}$ 
Cálculo

inércia em relação ao eixo y :

$\mathrm{I}:=\frac{\mathrm{h} \cdot \mathrm{b}^{3}}{12}$

$\mathrm{I}=2.81 \times 10^{-2} \cdot \mathrm{m}^{4}$

massa linear :

$\mathrm{m}_{\mathrm{I}}:=\frac{\mathrm{h} \cdot \mathrm{b} \cdot \gamma_{\mathrm{c}}}{\mathrm{g}} \quad \mathrm{m}_{\mathrm{l}}=1.53 \times 10^{3} \frac{\mathrm{kg}}{\mathrm{m}}$

massa generalizada :

$\mathrm{m}_{\mathrm{e}}:=\left(\frac{3}{2}-\frac{4}{\pi}\right) \cdot \mathrm{m}_{\mathrm{l}} \cdot \mathrm{L} \quad \mathrm{m}_{\mathrm{e}}=693.69 \mathrm{~kg}$

rigidez a flexão generalizada :

$K_{f}:=\frac{\pi^{4} \cdot E \cdot I}{32 \cdot L^{3}} \quad K_{f}=3.75 \times 10^{8} \cdot \frac{N}{m}$

rigidez geométrica generalizada :

$K_{g}:=\frac{\pi^{2} \cdot P}{8 \cdot L}$

$\mathrm{K}_{\mathrm{g}}=8.80 \times 10^{3} \cdot \frac{\mathrm{N}}{\mathrm{m}}$

rigidez generalizada :

$K:=K_{f}-K_{g}$

$\mathrm{K}=3.75 \times 10^{8} \cdot \frac{\mathrm{N}}{\mathrm{m}}$

d) Características do Sistema

massa total :

$M_{0}:=m_{m}+m_{e}$

$\mathrm{M}_{0}=2.15 \times 10^{3} \mathrm{~kg}$

freqüência natural :

$\omega:=\sqrt{\frac{\mathrm{K}}{\mathrm{M}_{0}}}$

$\omega=417.51 \cdot \frac{\mathrm{rad}}{\mathrm{s}}$

$f_{0}:=\frac{\omega}{2 \cdot \pi}$

$f_{0}=66.45 \frac{1}{s}$

taxa de amortecimento :

$\xi:=0.05$

amortecimento :

$\mathrm{C}:=2 \cdot \mathrm{M}_{0} \cdot \omega \cdot \xi$

$\mathrm{C}=8.97 \times 10^{4} \frac{\mathrm{kg}}{\mathrm{s}}$

excentricidade :

$r:=e$

$r=3.36 \times 10^{-5} \mathrm{~m}$

massa desbalanceada :

$m_{d}:=\frac{m_{g} \cdot e}{r} \quad m_{d}=946 k g$

parâmetros auxiliares :

$J_{0}:=m_{d} \cdot r^{2}+J_{m} \quad J_{0}=6.34 \cdot \mathrm{kg} \cdot \mathrm{m}^{2}$

$\mathrm{S}_{0}:=\mathrm{m}_{\mathrm{d}} \cdot \mathrm{r}$

$S_{0}=3.18 \times 10^{-2} \cdot \mathrm{kg} \cdot \mathrm{m}$ 
Parte II - Método Runge Kutta com Passo Fixo

a) Intervalo de Integrȩão

intervalo :

inicio $:=0$

fim $:=200$

número de partes por ciclo :

$p:=20 s$

número de passos :

npasso $:=\frac{\text { fim }- \text { inicio }}{\frac{1}{p} \cdot \frac{1}{f_{0}}}$

número de passos calculado :

npasso $=2.658 \times 10^{5}$

número de passos fixado :

npasso $:=260000$

b) Condições Iniciais

aceleração estrutura :

$v_{\mathrm{pp}}:=0$

aceleração massa desbalanceada : $\quad \phi_{\mathrm{pp}}:=0$

velocidade estrutura :

$v_{p}:=0$

rotação do motor :

$\phi_{p}:=0$

c) Vetor Condições Iniciais

Cond $_{i n i}:=\left(\begin{array}{c}v_{p} \\ \phi_{p} \\ v_{p p} \\ \phi_{p p}\end{array}\right)$ 
d) Coeficientes do Polinômio $12^{\circ}$ grau - Curva Caracteística Líquida (L-H)

a12 := -0.00000000000000000005079203355095821

a11 $:=0.00000000000000005158768499255971$

$\mathrm{a} 10:=-0.000000000000022936931310257087$

a9 $:=0.0000000000058675559999675535$

a8 $:=-0.0000000009548054572554488$

a7 := 0.00000010320363974351303

$\mathrm{a} 6:=-0.000007515971501257237$

$\mathrm{a} 5:=0.0003664916514154084$

a4 := $=0.01164087119466062$

a3 $:=0.22641715279886516$

a2 $:=-2.320439888509873$

a1 $:=2.7922627314204136$

$\mathrm{a} 0:=2587.2836399918815$

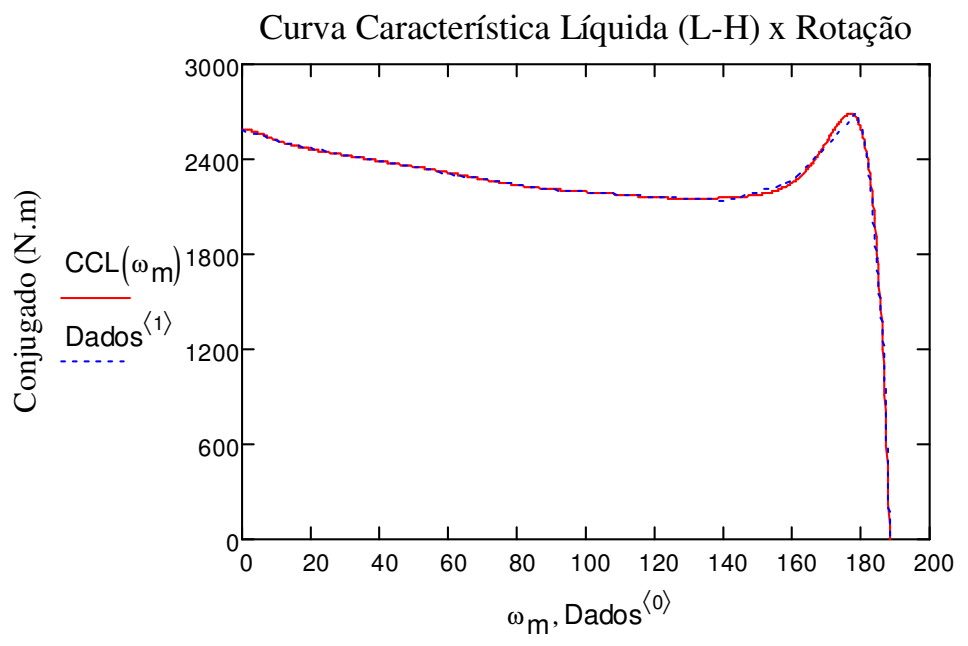

Rotação ( $\mathrm{rad} / \mathrm{s})$ 
e) Vetor Derivada $D(t, R)$

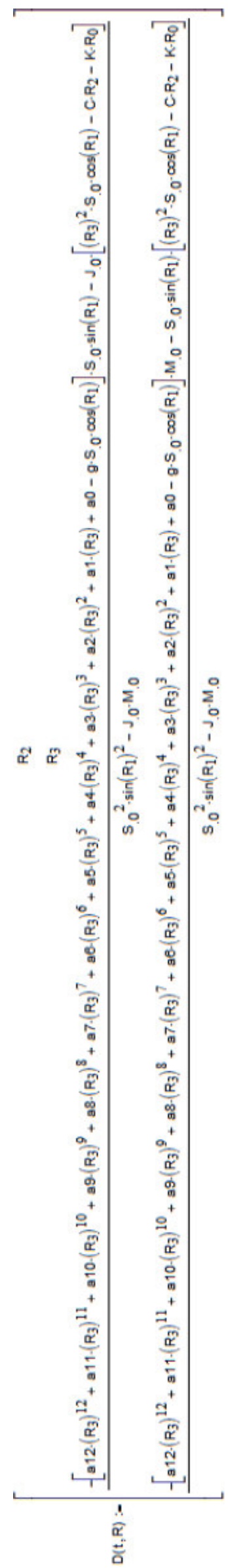


$S:=\operatorname{rkfixed}\left(\right.$ Cond $_{\text {ini }}$, inicio , fim , npasso, D)

$\mathrm{t}:=\mathrm{S}^{\langle 0\rangle} \quad \operatorname{des}_{\text {sni }}:=\mathrm{S}^{\langle 1\rangle} \quad \operatorname{ang}_{\mathrm{sni}}:=\mathrm{S}^{\langle 2\rangle} \quad$ vel $_{\text {sni }}:=\mathrm{S}^{\langle 3\rangle} \quad{ }^{\langle 3} \mathrm{m}:=\mathrm{S}^{\langle 4\rangle}$

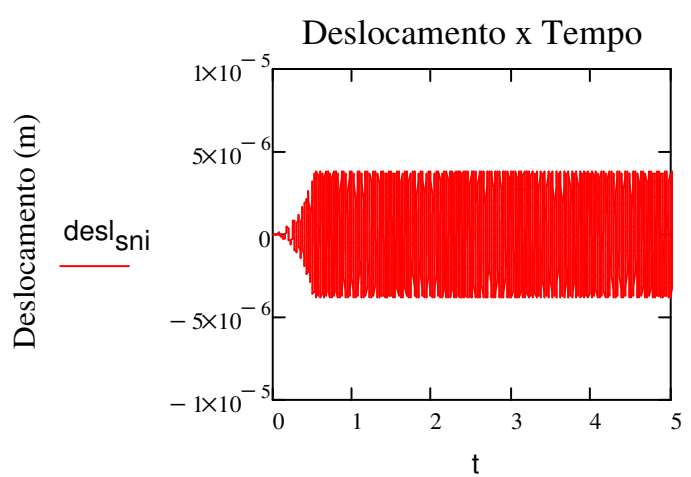

Tempo (s)

$\max \left(\operatorname{des}_{\text {Sni }}\right)=3.788 \times 10^{-6}$

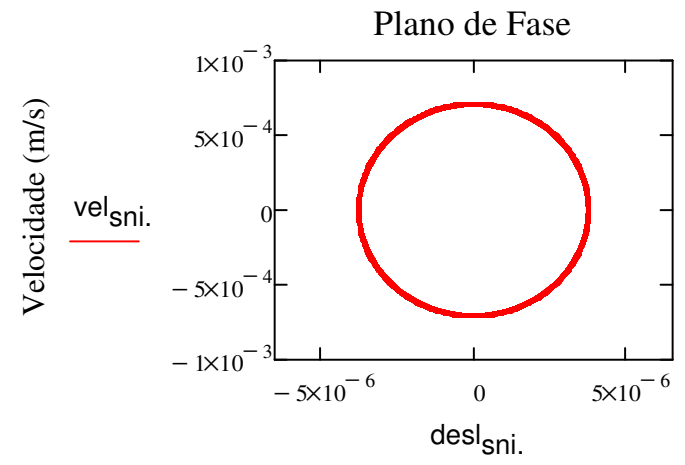

Deslocamento (m)

Ângulo x Tempo

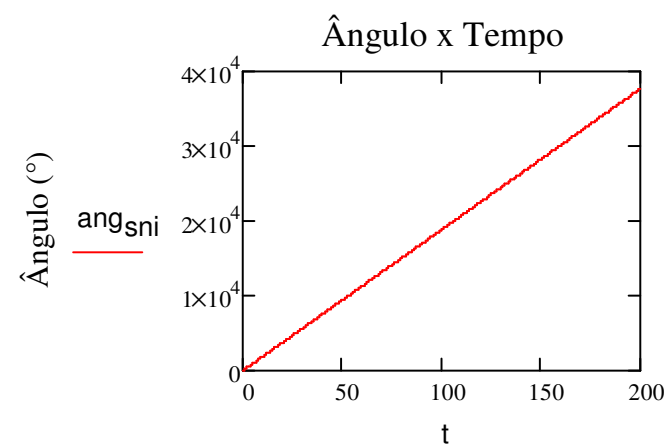

Tempo (s)

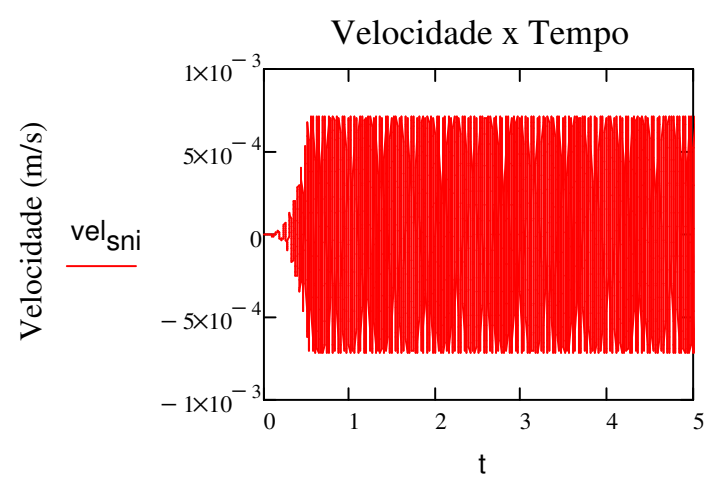

Tempo (s)

$\max \left(\right.$ vel $\left._{\text {sni }}\right)=7.145 \times 10^{-4}$

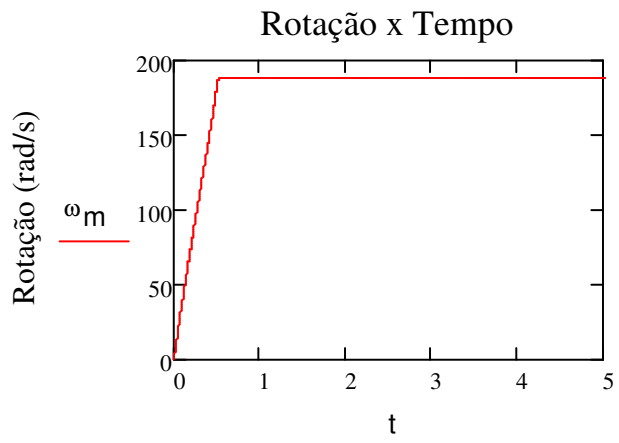

Tempo (s)

$\max \left(\omega_{\mathrm{m}}\right)=188.525$ 
Parte III - Cálculo da Energia Consumida pela Estrutura

rotação do motor :

$$
\omega_{\mathrm{m}}:=0.01,0.5 . .190
$$

relação entrefreqüências :

$$
\beta\left(\omega_{m}\right):=\frac{\omega_{m}}{\omega}
$$

fator de amplificação dinâmica :

$$
\mathrm{D}\left(\omega_{\mathrm{m}}\right):=\frac{1}{\sqrt{\left(1-\beta\left(\omega_{\mathrm{m}}\right)^{2}\right)^{2}+\left(2 \cdot \xi \cdot \beta\left(\omega_{\mathrm{m}}\right)\right)^{2}}}
$$

força desbalanceamento :

$$
\mathrm{P}\left(\omega_{\mathrm{m}}\right):=\mathrm{m}_{\mathrm{d}} \cdot \mathrm{r} \cdot \omega_{\mathrm{m}}{ }^{2}
$$

amplitude do movimento :

$$
\rho\left(\omega_{m}\right):=\frac{P\left(\omega_{m}\right)}{K} \cdot D\left(\omega_{m}\right)
$$

energia consumida :

$$
\mathrm{E}\left(\omega_{\mathrm{m}}\right):=\frac{\mathrm{C}}{2 \cdot \omega_{\mathrm{m}}} \cdot \omega^{2} \cdot \rho\left(\omega_{\mathrm{m}}\right)^{2}
$$

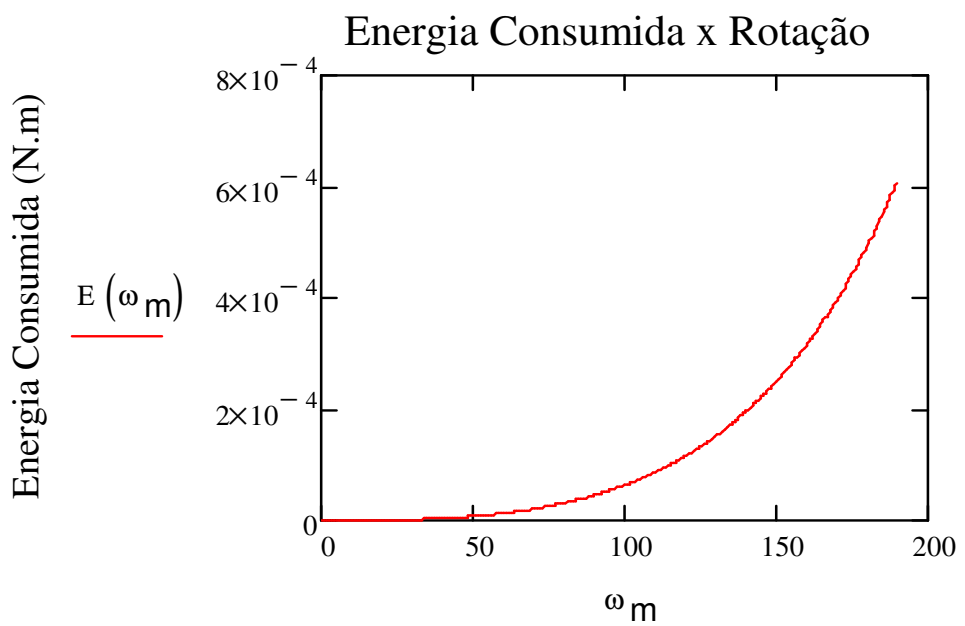

Rotação (rad/s) 
APÊNDICE D - GRÁFICOS DAS RESPOSTAS DA ESTRUTURA PARA SISTEMA NÃO-IDEAL DE ENERGIA

GRÁFICOS D.1 A D.10 - RESPOSTAS DA ESTRUTURA

Geometria da Estrutura

Largura $\quad b=0.75 m$

Comprimento $\quad h=0.80 \mathrm{~m}$

Altura $\quad \mathrm{L}=\operatorname{variável}(1.50 \mathrm{~m}$ a $6.00 \mathrm{~m})$

GRÁFICOS D.11 A D.20 - RESPOSTAS DA ESTRUTURA

Geometria da Estrutura

Largura $\quad b=0.75 m$

Comprimento $\quad h=1.00 m$

Altura $\quad \mathrm{L}=\operatorname{variável}(1.50 \mathrm{~m}$ a $6.00 \mathrm{~m})$

GRÁFICOS D.21 A D.30 - RESPOSTAS DA ESTRUTURA

Geometria da Estrutura

Largura $\quad b=0.95 \mathrm{~m}$

Comprimento $\quad h=0.80 m$

Altura $\quad \mathrm{L}=\operatorname{variável}(1.50 \mathrm{~m}$ a $6.00 \mathrm{~m})$ 
Gráficos D. 1 - Respostas da estrutura $b=0.75 \mathrm{~m}, \mathrm{~h}=0.80 \mathrm{~m}$ e $\mathrm{L}=1.5 \mathrm{~m}$

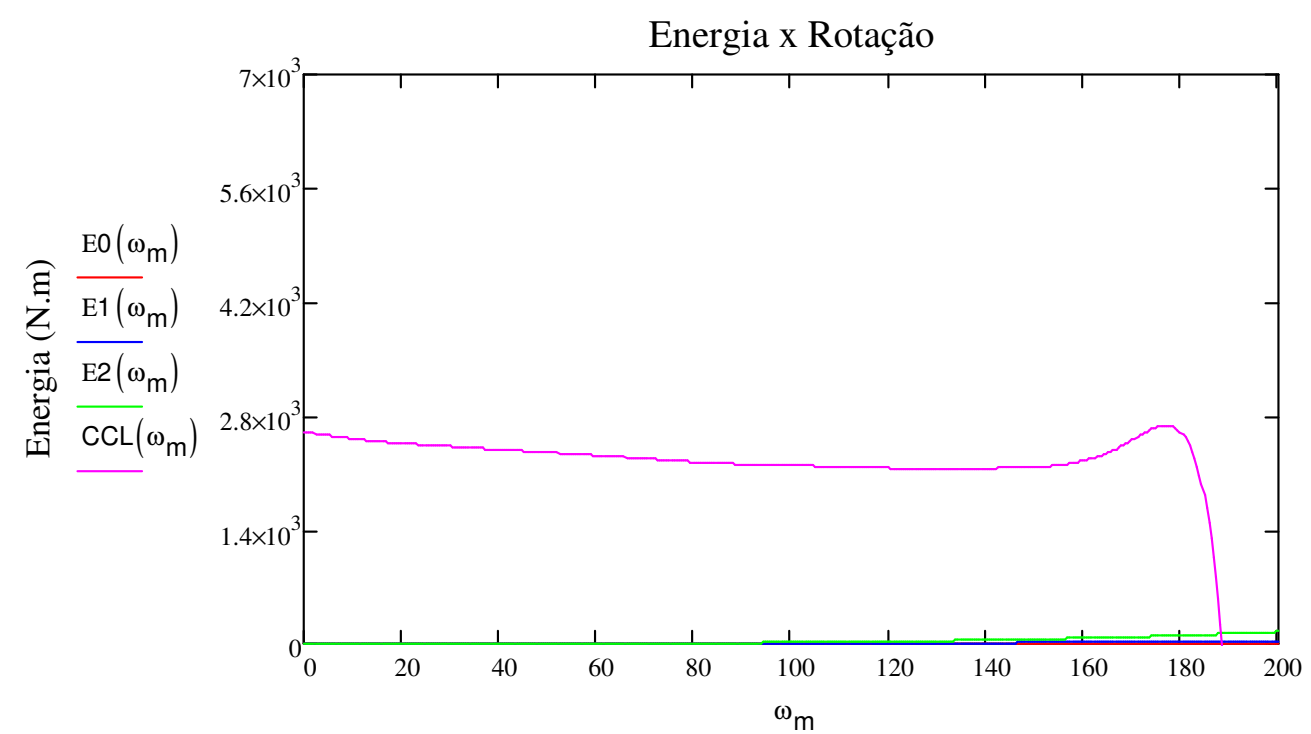

Rotação (rad/s)

— Curva de Energia Consumida pela Estrutura para r0 : $0.034 \mathrm{~mm}$

_ Curva de Energia Consumida pela Estrutura para r1 : $10.67 \mathrm{~mm}$

Curva de Energia Consumida pela Estrutura para r2: $21.34 \mathrm{~mm}$

Curva Característica Líquida do Motor (L-H)

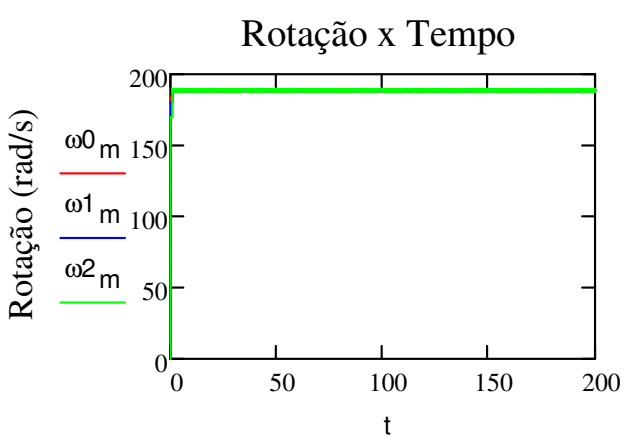

Tempo (s)

Deslocamento x Tempo

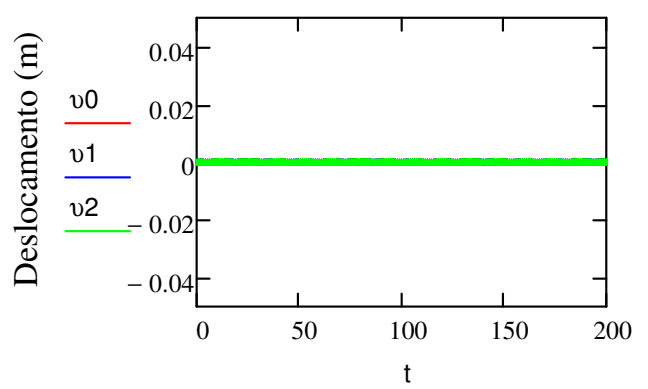

Tempo (s)

$$
\mathrm{E0}_{\max }:=3.67 \cdot 10^{-4}
$$$$
\mathrm{E} 1 \text { max }:=36.975
$$$$
\mathrm{E} 2 \text { max }:=147.90
$$

Rotação x Tempo

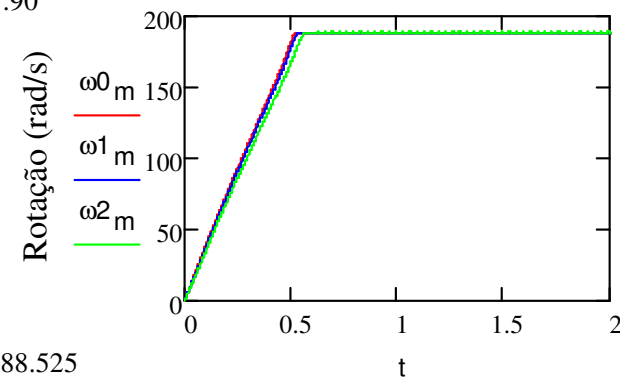

$\max \left(\omega_{\mathrm{m}}\right)=188.525$

$\max (\omega 1 \mathrm{~m})=188.593$

$\max \left(\omega 2_{m}\right)=188.691$

Tempo (s)

Deslocamento x Tempo

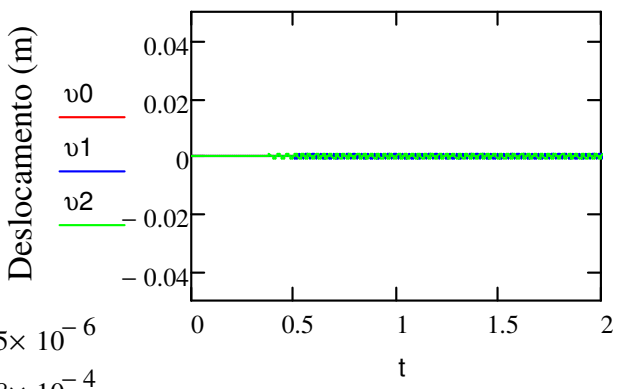

$\max (v 1)=4.398 \times 10^{-4}$

$\max (v 2)=8.818 \times 10^{-4}$

Tempo (s) 
Gráficos D.2 - Respostas da estrutura $b=0.75 \mathrm{~m}, \mathrm{~h}=0.80 \mathrm{~m}$ e $\mathrm{L}=2.0 \mathrm{~m}$

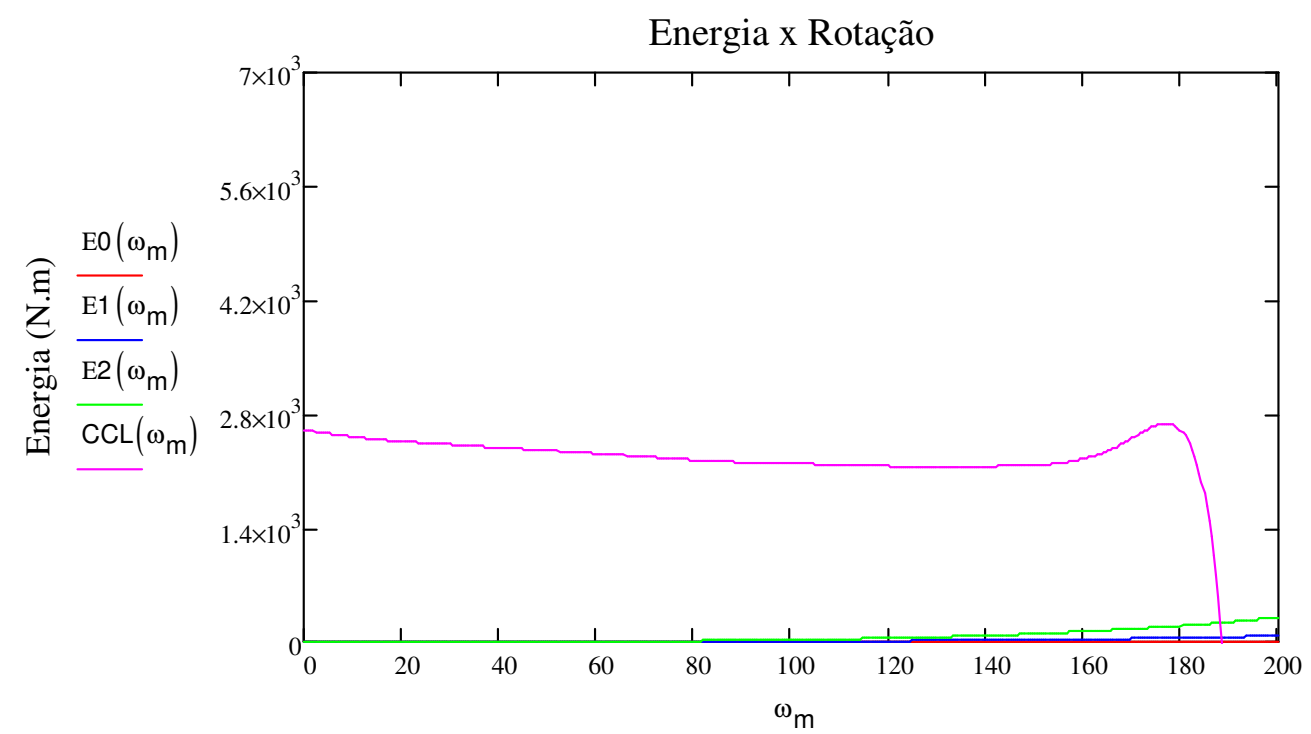

Rotação (rad/s)

— Curva de Energia Consumida pela Estrutura para r0 : $0.034 \mathrm{~mm}$

_ Curva de Energia Consumida pela Estrutura para r1 : $10.67 \mathrm{~mm}$

Curva de Energia Consumida pela Estrutura para r2: $21.34 \mathrm{~mm}$

Curva Característica Líquida do Motor (L-H)

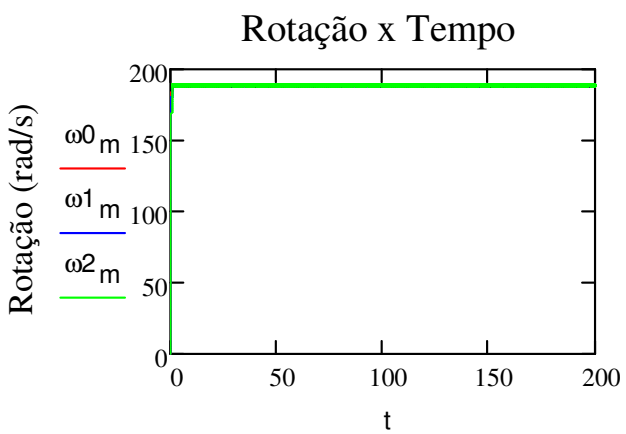

Tempo (s)

Deslocamento x Tempo

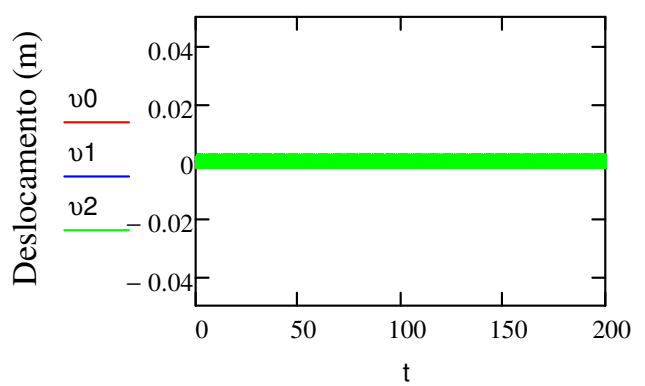

Tempo (s)
$\mathrm{E}_{\max }:=7.55 \cdot 10^{-4}$

$\mathrm{E}_{\text {max }}:=76.065$

$\mathrm{E}_{\text {max }}:=324.25$

Rotação x Tempo

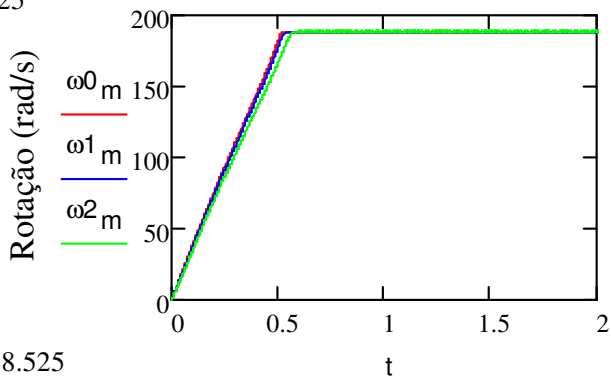

$\max \left(\omega 0_{\mathrm{m}}\right)=188.525$

$\max (\omega 1 \mathrm{~m})=188.625$

$\max (\omega 2 \mathrm{~m})=188.829$

Tempo (s)

Deslocamento x Tempo

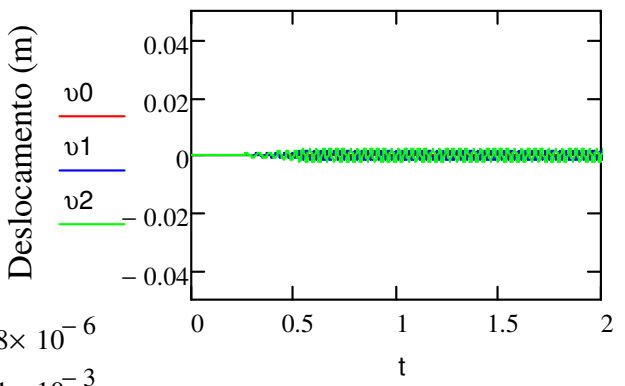

Tempo (s) $\max (v 1)=1.201 \times 10^{-3}$

$\max (v 2)=2.393 \times 10^{-3}$ 
Gráficos D.3 - Respostas da estrutura $b=0.75 \mathrm{~m}, \mathrm{~h}=0.80 \mathrm{~m}$ e $\mathrm{L}=2.5 \mathrm{~m}$

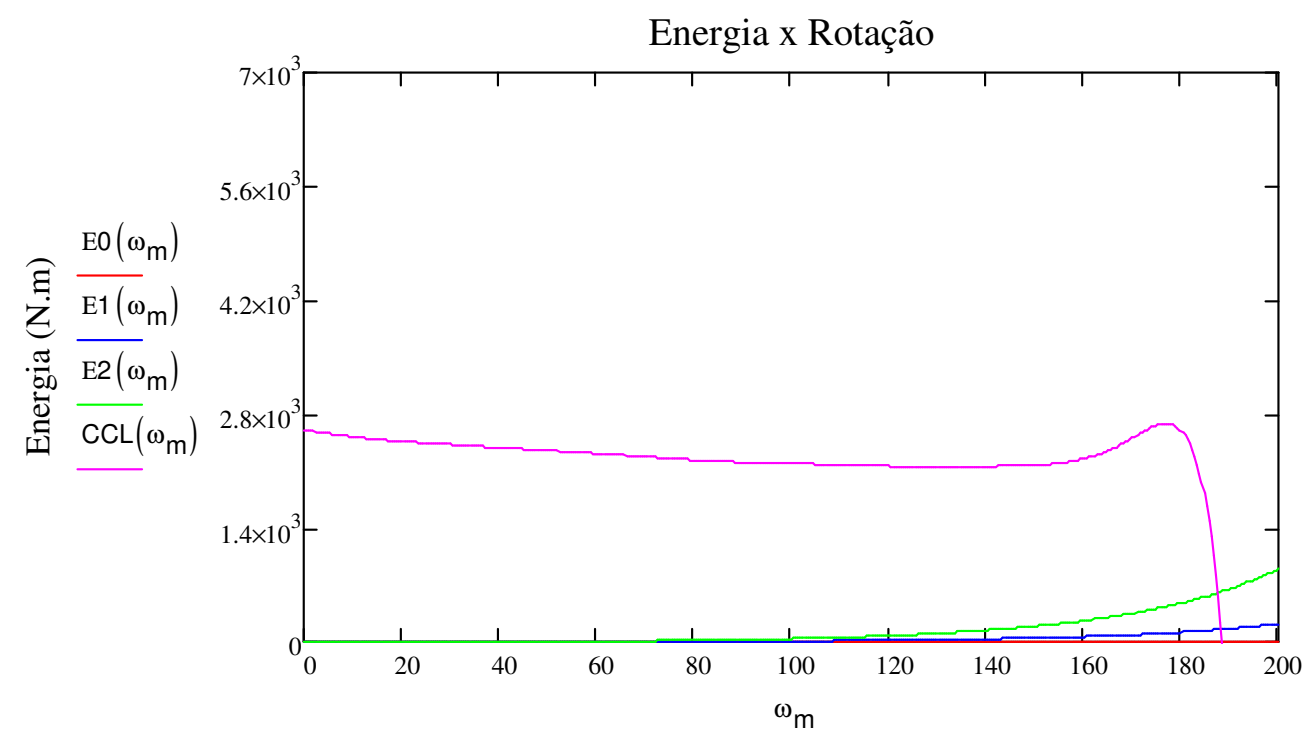

Rotação (rad/s)

— Curva de Energia Consumida pela Estrutura para r0 : $0.034 \mathrm{~mm}$

_ Curva de Energia Consumida pela Estrutura para r1 : $10.67 \mathrm{~mm}$

Curva de Energia Consumida pela Estrutura para r2: $21.34 \mathrm{~mm}$

Curva Característica Líquida do Motor (L-H)

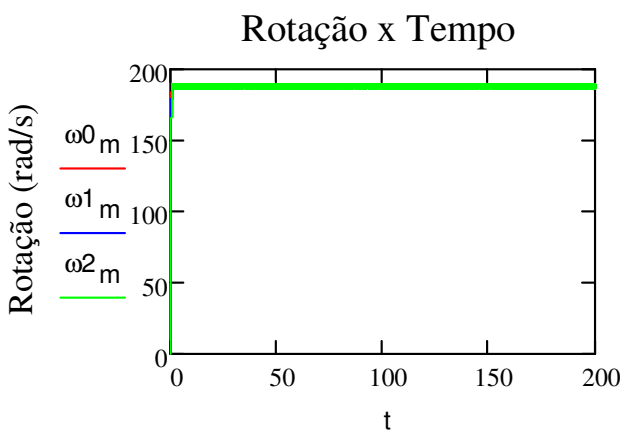

Tempo (s)

Deslocamento x Tempo

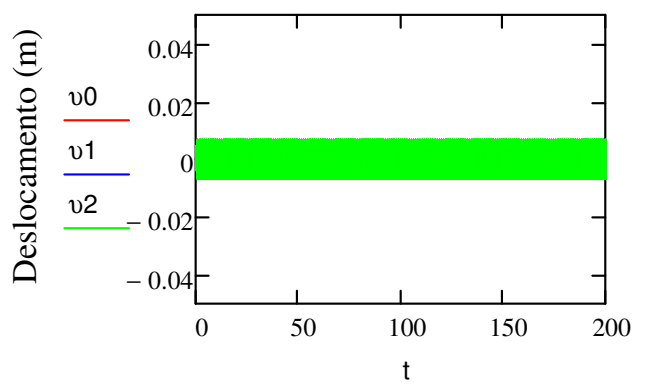

Tempo (s)

$$
\mathrm{E} 0_{\max }:=2.23 \cdot 10^{-3}
$$$$
\mathrm{E1}_{\text {max }}:=224.93
$$$$
\mathrm{E}_{\text {max }} \text { := } 899.70
$$

Rotação x Tempo

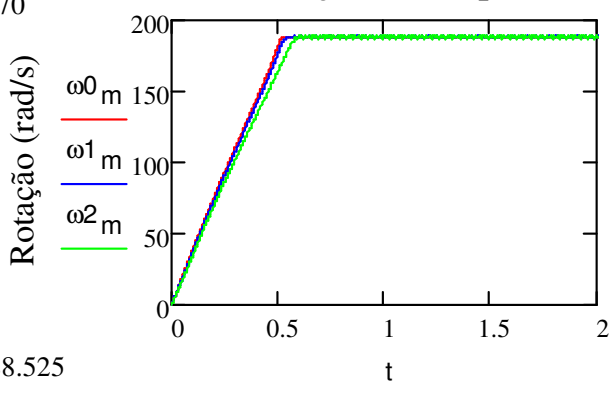

$\max \left(\omega 0_{\mathrm{m}}\right)=188.525$

$\max \left(\omega 1^{m}\right)=188.69$

$\max \left(\omega 2_{m}\right)=189.038$

Tempo (s)

Deslocamento x Tempo

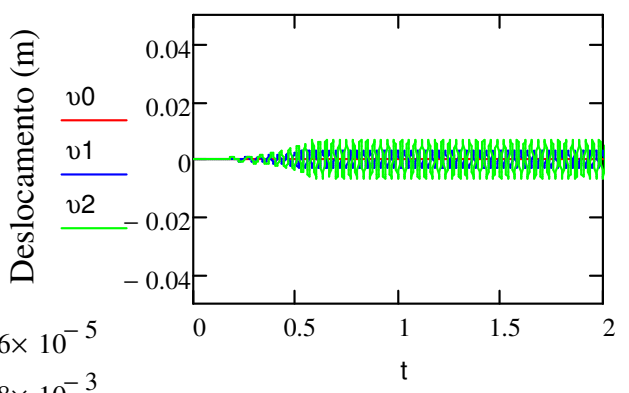

Tempo (s) $\max (v 1)=3.258 \times 10^{-3}$

$\max (v 2)=6.467 \times 10^{-3}$ 
Gráficos D.4 - Respostas da estrutura $b=0.75 \mathrm{~m}, \mathrm{~h}=0.80 \mathrm{~m}$ e $\mathrm{L}=3.0 \mathrm{~m}$

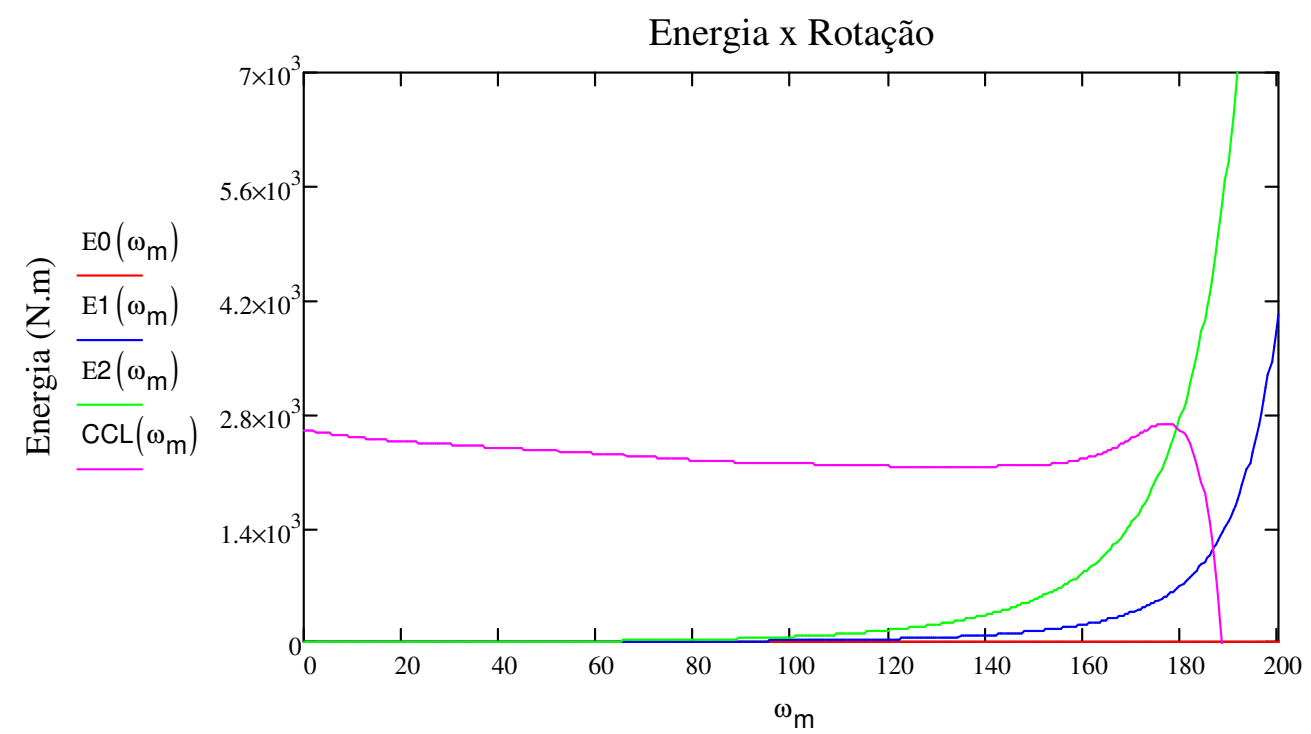

Rotação (rad/s)

— Curva de Energia Consumida pela Estrutura para r0 : $0.034 \mathrm{~mm}$

_ Curva de Energia Consumida pela Estrutura para r1 : $10.67 \mathrm{~mm}$

Curva de Energia Consumida pela Estrutura para r2: $21.34 \mathrm{~mm}$

Curva Característica Líquida do Motor (L-H)

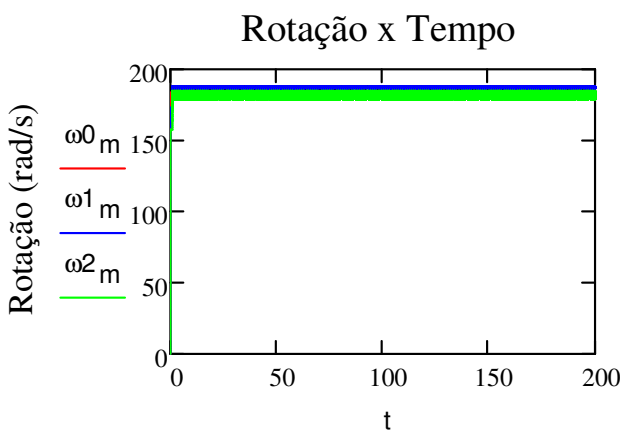

Tempo (s)

Deslocamento x Tempo

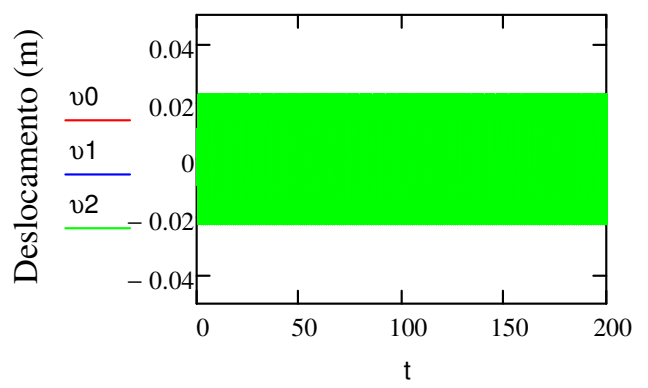

Tempo (s)

$$
\begin{aligned}
& \mathrm{E}_{\text {max }}:=0.04 \\
& \mathrm{E}_{\text {max }}:=4.02 \cdot 10^{3} \\
& { }^{\mathrm{E}} \text { max }_{\text {max }}:=1.61 \cdot 10^{4}
\end{aligned}
$$

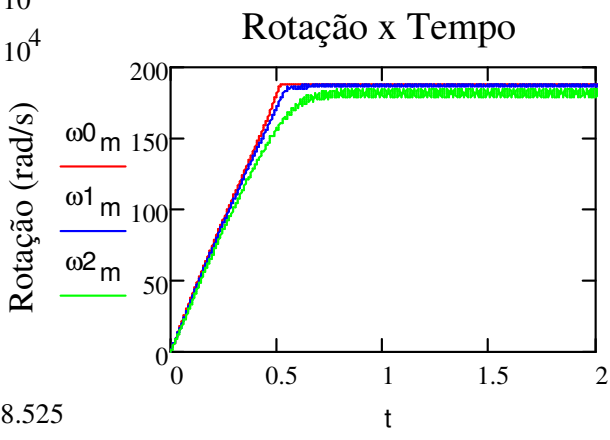

$\max \left(\omega 0_{\mathrm{m}}\right)=188.525$

$\max \left(\omega 1_{\mathrm{m}}\right)=188.07$

$\max \left(\omega 2_{m}\right)=184.815$

Tempo (s)

Deslocamento x Tempo

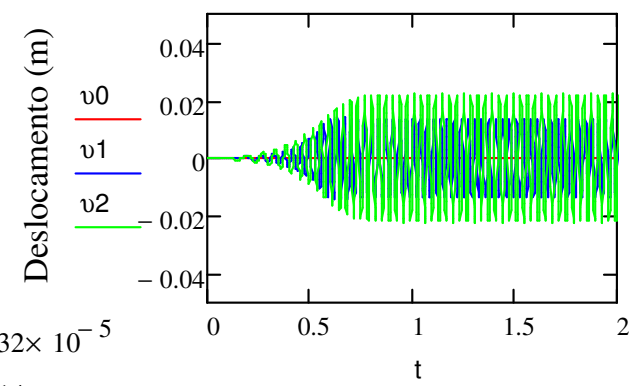

Tempo (s) 
Gráficos D.5 - Respostas da estrutura $b=0.75 \mathrm{~m}, \mathrm{~h}=0.80 \mathrm{~m}$ e $\mathrm{L}=3.5 \mathrm{~m}$

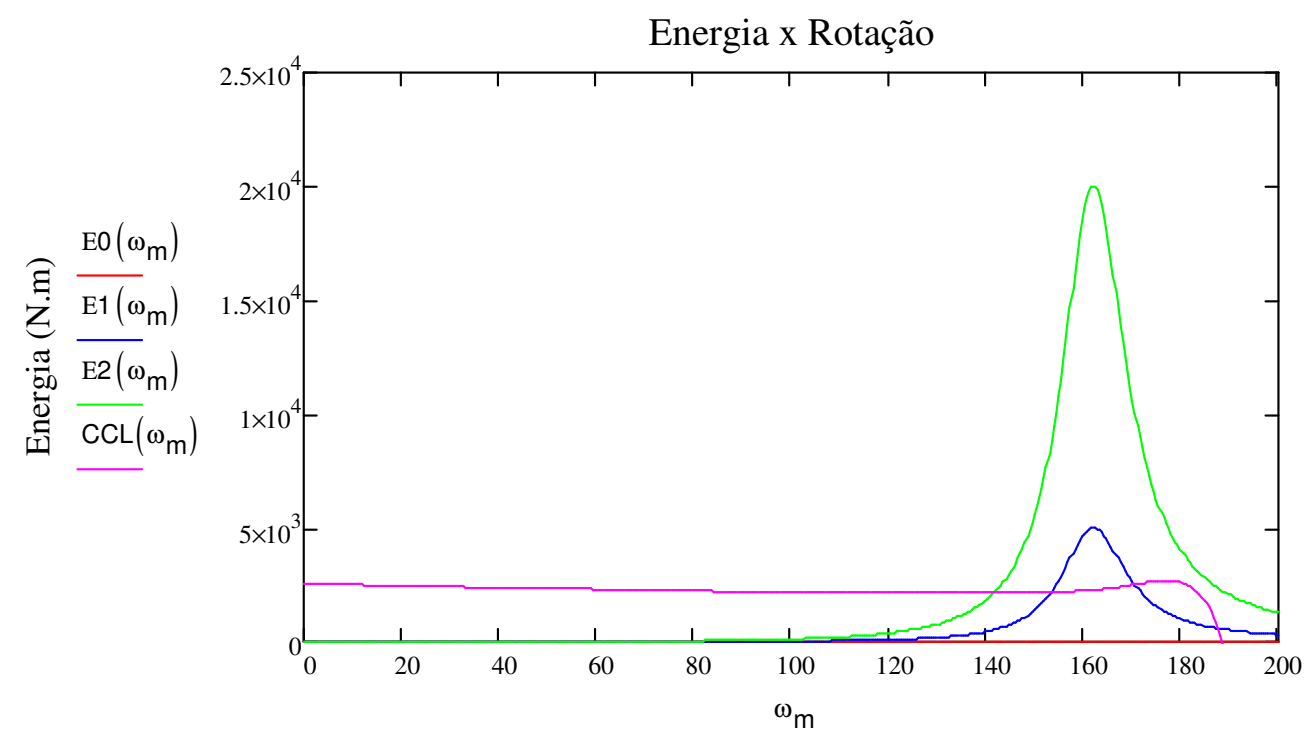

Rotação (rad/s)

— Curva de Energia Consumida pela Estrutura para r0 : $0.034 \mathrm{~mm}$

_ Curva de Energia Consumida pela Estrutura para r1 : $10.67 \mathrm{~mm}$

Curva de Energia Consumida pela Estrutura para r2: $21.34 \mathrm{~mm}$ Curva Característica Líquida do Motor (L-H)

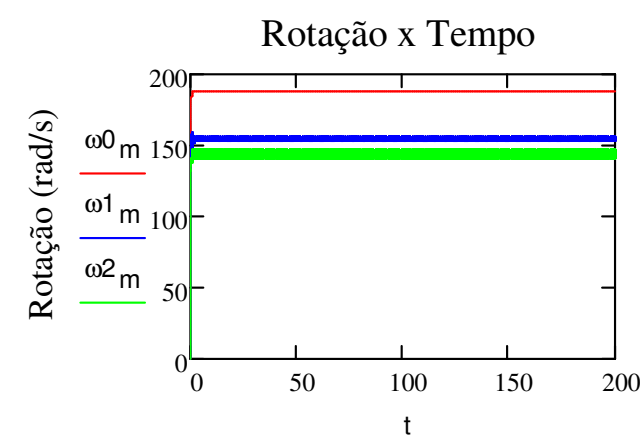

Tempo (s)

Deslocamento x Tempo

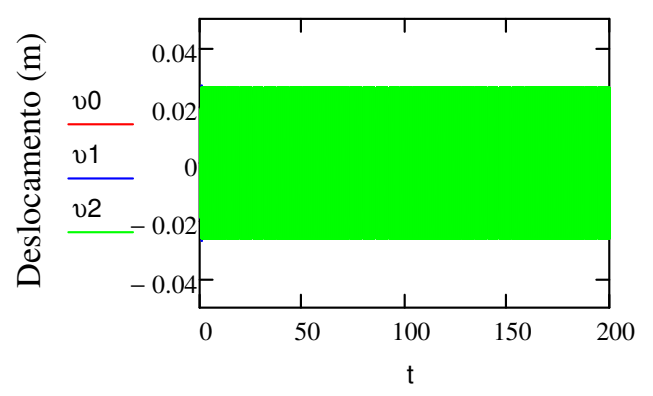

Tempo (s)
$\mathrm{E}_{\text {max }}:=0.05$
${ }^{\mathrm{E} 1}$ max
$\mathrm{E}_{\text {max }}:=5.0 \cdot 10^{3}$
$=2.0 \cdot 10^{4}$

$$
\mathrm{E} 2_{\text {max }}:=2.0 \cdot 10^{4}
$$

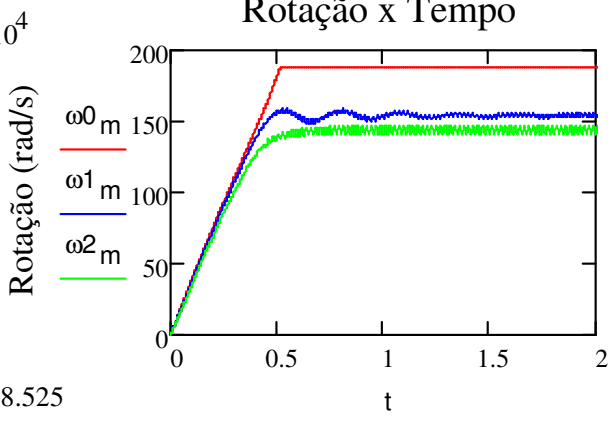

$\max \left(\omega 0_{\mathrm{m}}\right)=188.525$

$\max \left(\omega 1_{\mathrm{m}}\right)=158.535$

$\max \left(\omega 2_{m}\right)=146.957$

Tempo (s)

Deslocamento x Tempo

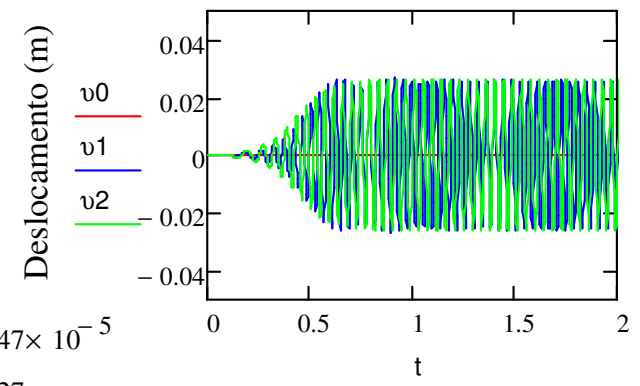

Tempo (s) 
Gráficos D.6 - Respostas da estrutura $b=0.75 \mathrm{~m}, \mathrm{~h}=0.80 \mathrm{~m}$ e $\mathrm{L}=4.0 \mathrm{~m}$

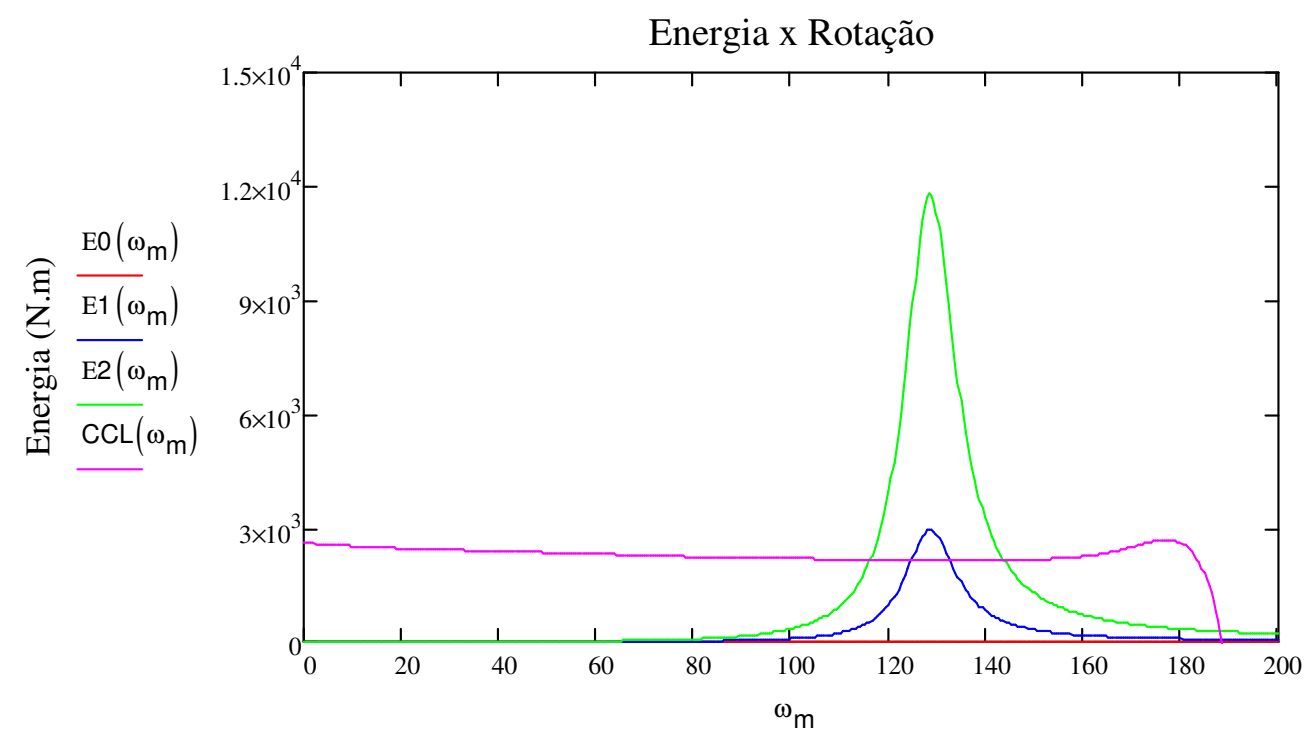

Rotação (rad/s)

_ Curva de Energia Consumida pela Estrutura para r0 : $0.034 \mathrm{~mm}$

_ Curva de Energia Consumida pela Estrutura para r1 : $10.67 \mathrm{~mm}$

Curva de Energia Consumida pela Estrutura para r2: $21.34 \mathrm{~mm}$ Curva Característica Líquida do Motor (L-H)

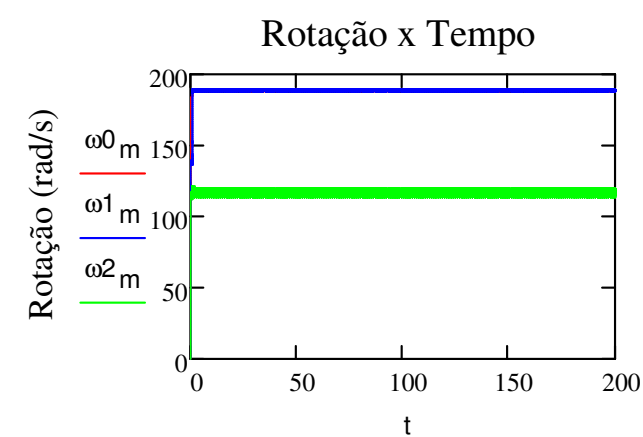

Tempo (s)

Deslocamento x Tempo

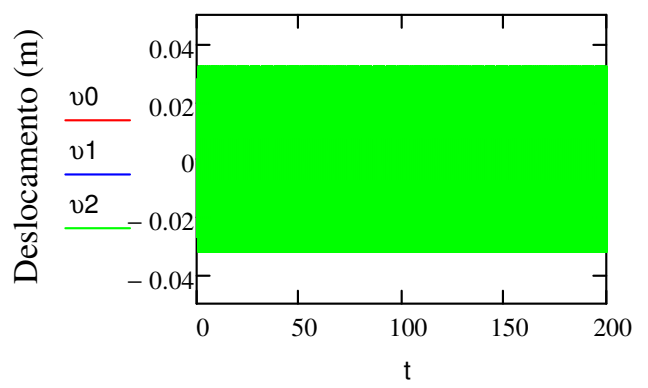

Tempo (s)
$\mathrm{E} 0_{\text {max }}:=0.029$
$\mathrm{E}_{\text {max }}:=2.95 \cdot 10^{3}$
${ }^{\mathrm{E} 2_{\text {max }}}:=1.18 \cdot 10^{4}$

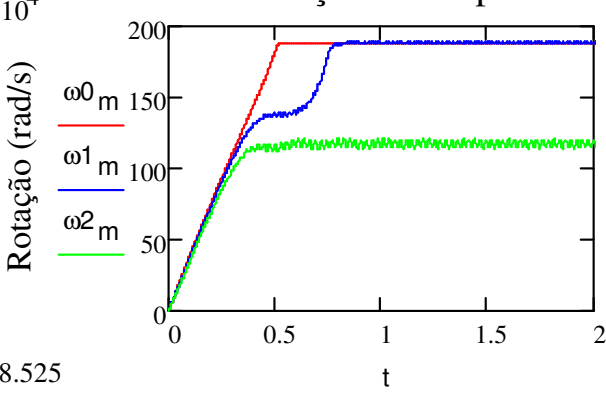

$\max \left(\omega 0_{\mathrm{m}}\right)=188.525$

$\max (\omega 1 \mathrm{~m})=189.213$

$\max \left(\omega 2_{m}\right)=120.9$

Tempo (s)

Deslocamento x Tempo

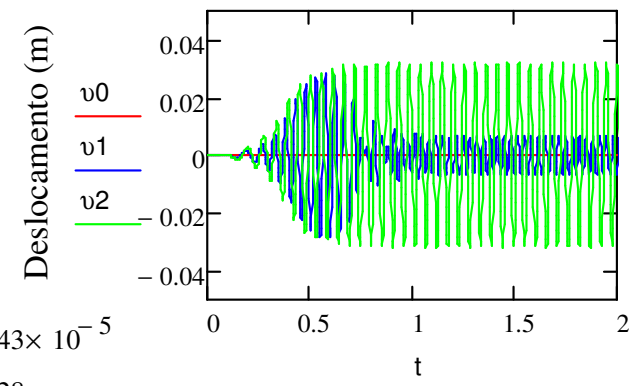

Tempo (s) 
Gráficos D.7 - Respostas da estrutura $b=0.75 \mathrm{~m}, \mathrm{~h}=0.80 \mathrm{~m}$ e $\mathrm{L}=4.5 \mathrm{~m}$

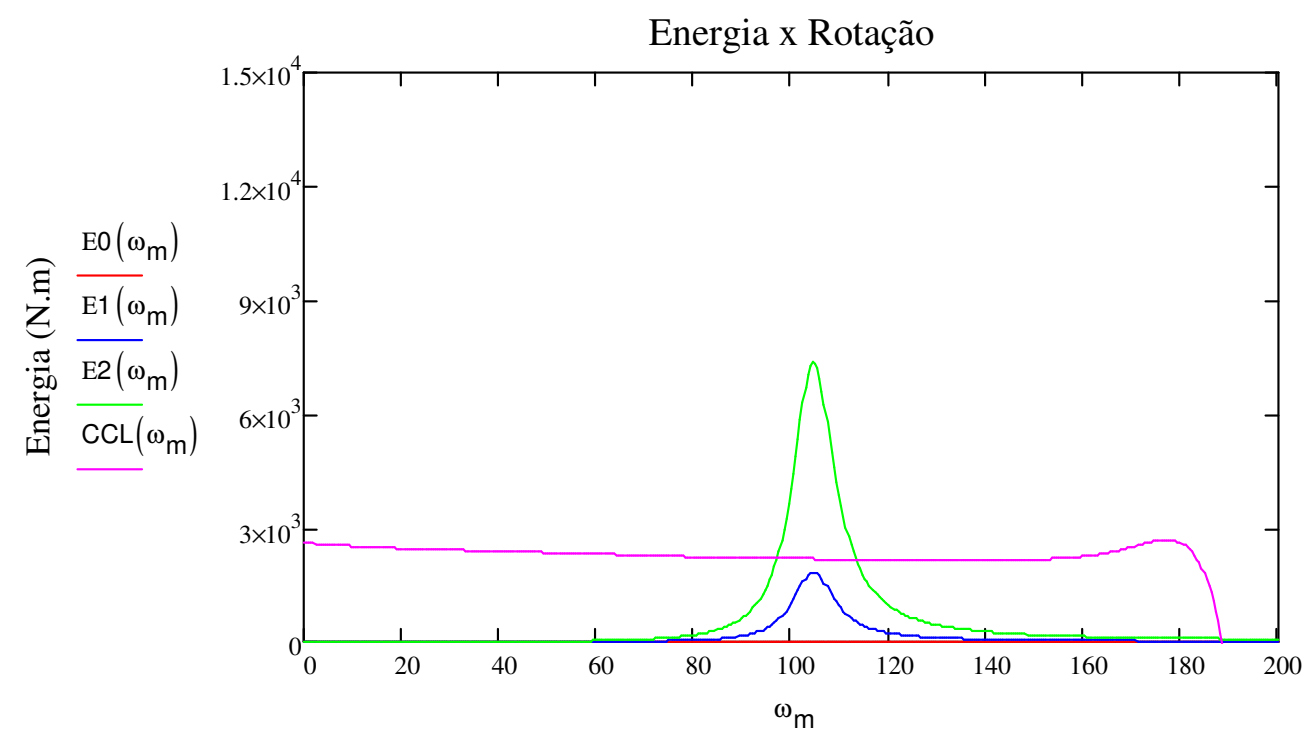

Rotação (rad/s)

_ Curva de Energia Consumida pela Estrutura para r0 : $0.034 \mathrm{~mm}$

_ Curva de Energia Consumida pela Estrutura para r1 : $10.67 \mathrm{~mm}$

Curva de Energia Consumida pela Estrutura para r2: $21.34 \mathrm{~mm}$ Curva Característica Líquida do Motor (L-H)

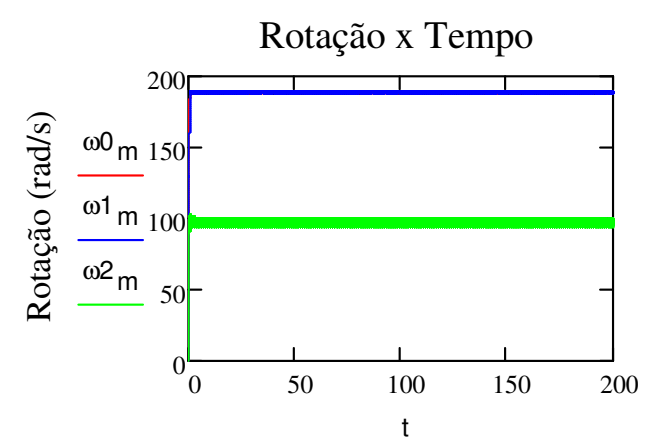

Tempo (s)

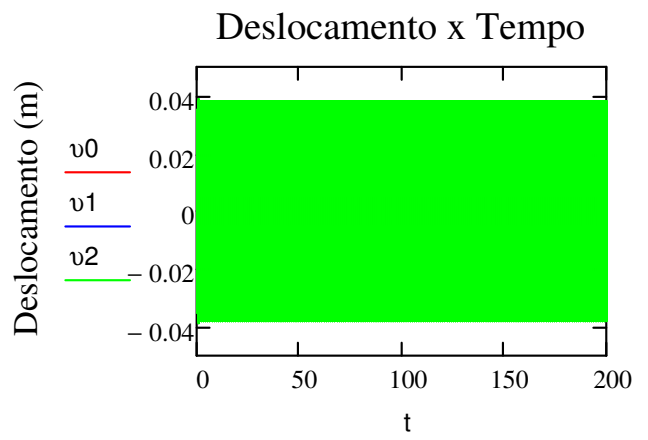

Tempo (s)

$$
\begin{aligned}
& \mathrm{E}_{\text {max }}:=0.018 \\
& \mathrm{E}_{\text {max }}:=1.84 \cdot 10^{3} \\
& { }^{\mathrm{E}}{ }_{\text {max }}:=7.36 \cdot 10^{3}
\end{aligned}
$$

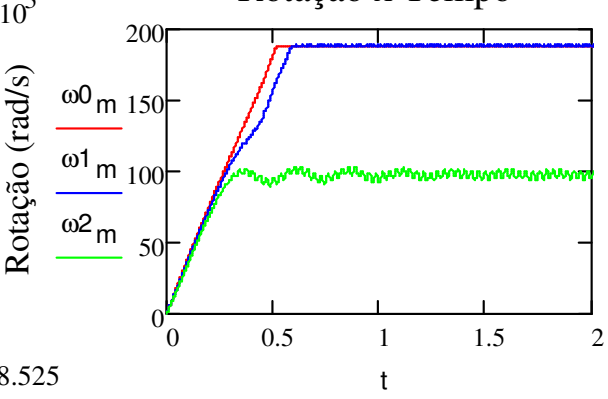

$\max \left(\omega 0_{\mathrm{m}}\right)=188.525$

$\max \left(\omega 1_{\mathrm{m}}\right)=188.965$

$\max (\omega 2 \mathrm{~m})=103.671$

Tempo (s)

Deslocamento x Tempo

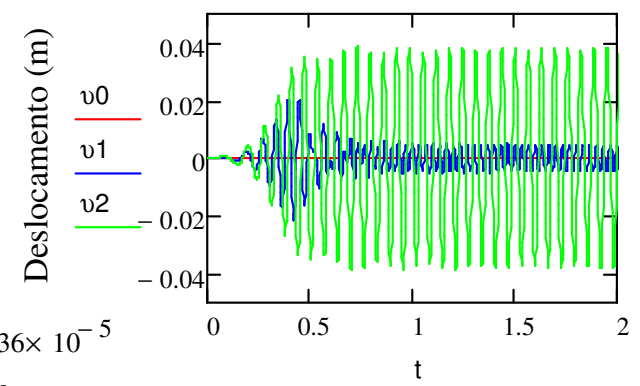

Tempo (s) 
Gráficos D.8 - Respostas da estrutura $b=0.75 \mathrm{~m}, \mathrm{~h}=0.80 \mathrm{~m}$ e $\mathrm{L}=5.0 \mathrm{~m}$

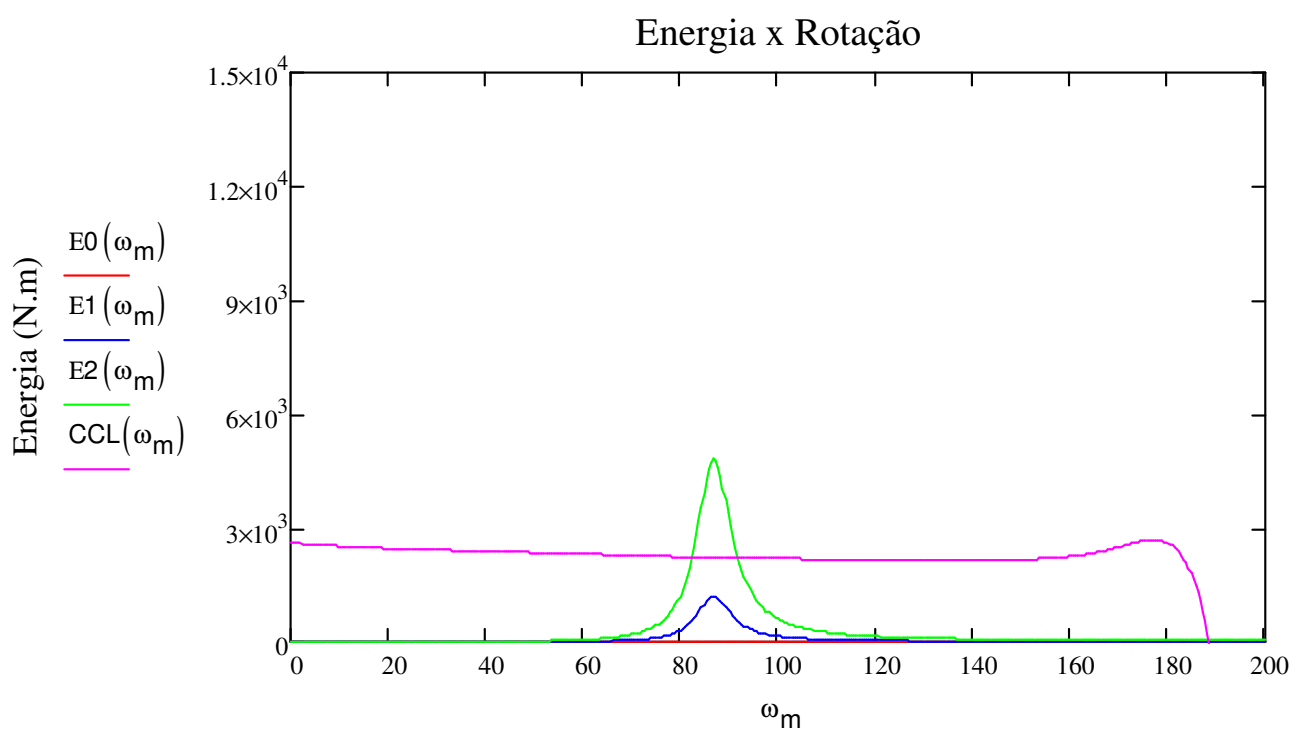

Rotação (rad/s)

— Curva de Energia Consumida pela Estrutura para r0 : $0.034 \mathrm{~mm}$

Curva de Energia Consumida pela Estrutura para r1 : $10.67 \mathrm{~mm}$

Curva de Energia Consumida pela Estrutura para r2 : $21.34 \mathrm{~mm}$

Curva Característica Líquida do Motor (L-H)

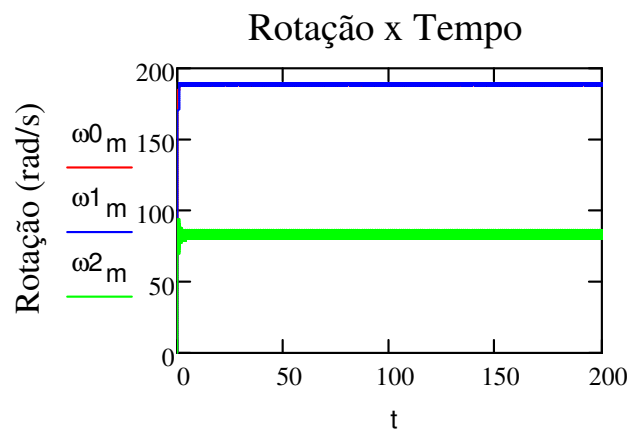

Tempo (s)

Deslocamento x Tempo

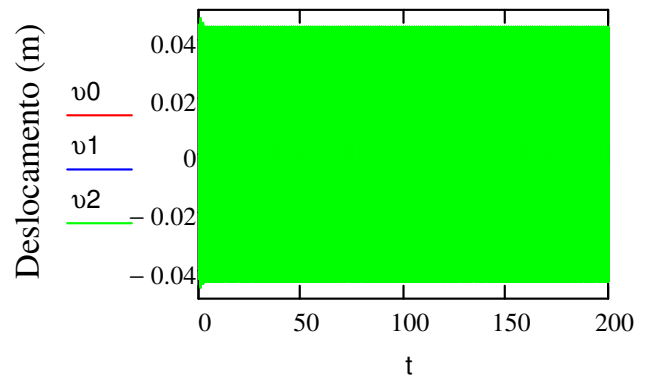

Tempo (s)

$$
\begin{aligned}
& \mathrm{E}_{\text {max }}:=0.012 \\
& \mathrm{E}_{\text {max }}:=1.2 \cdot 10^{3} \\
& \mathrm{E}_{\text {max }}:=4.8 \cdot 10^{3}
\end{aligned}
$$

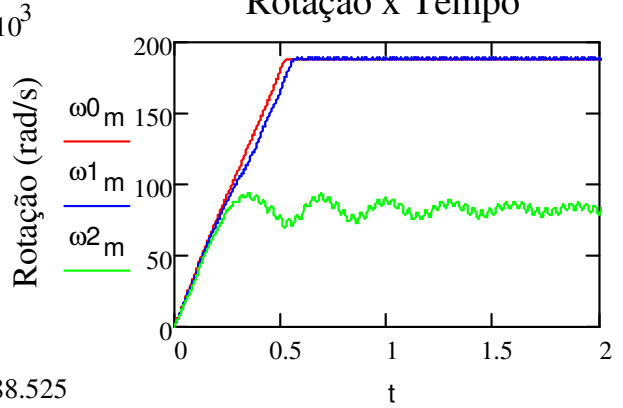

$\max \left(\omega 0_{\mathrm{m}}\right)=188.525$

$\max \left(\omega 1_{\mathrm{m}}\right)=188.906$

$\max \left(\omega 2_{m}\right)=93.555$

Tempo (s)

Deslocamento x Tempo

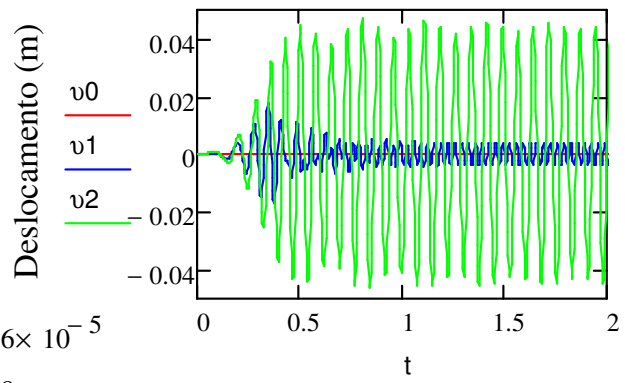

Tempo (s) 
Gráficos D.9 - Respostas da estrutura $b=0.75 \mathrm{~m}, \mathrm{~h}=0.80 \mathrm{~m}$ e $\mathrm{L}=5.5 \mathrm{~m}$

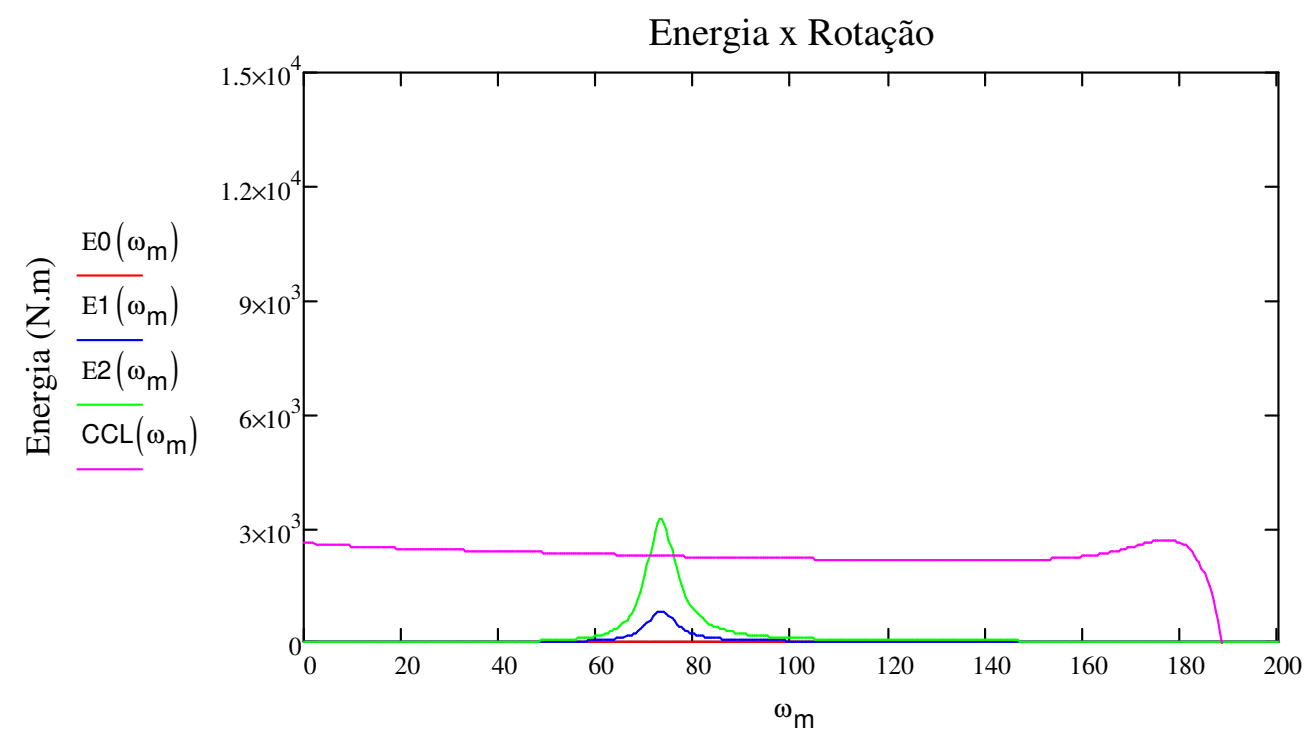

Rotação (rad/s)

— Curva de Energia Consumida pela Estrutura para r0 : $0.034 \mathrm{~mm}$

_ Curva de Energia Consumida pela Estrutura para r1 : $10.67 \mathrm{~mm}$

Curva de Energia Consumida pela Estrutura para r2: $21.34 \mathrm{~mm}$

Curva Característica Líquida do Motor (L-H)

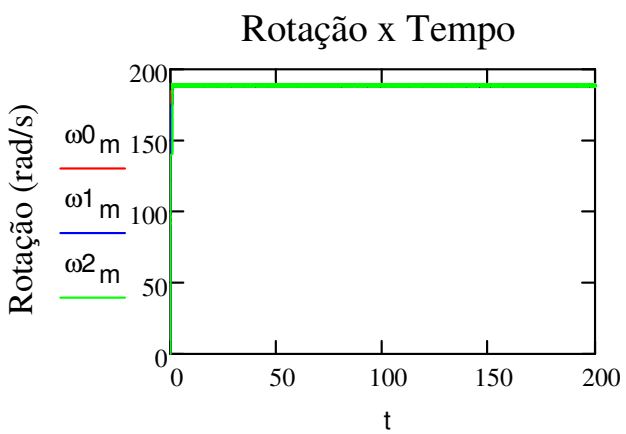

Tempo (s)

Deslocamento x Tempo

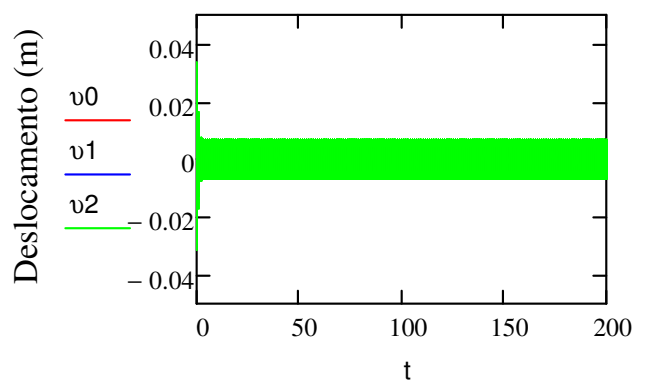

Tempo (s)

$$
\mathrm{E}_{\text {max }}:=8.02 \cdot 10^{-3}
$$$$
\mathrm{E}_{\text {max }}:=807.89
$$$$
\mathrm{E}_{\text {max }}:=3.23 \cdot 10^{3}
$$

Rotação x Tempo

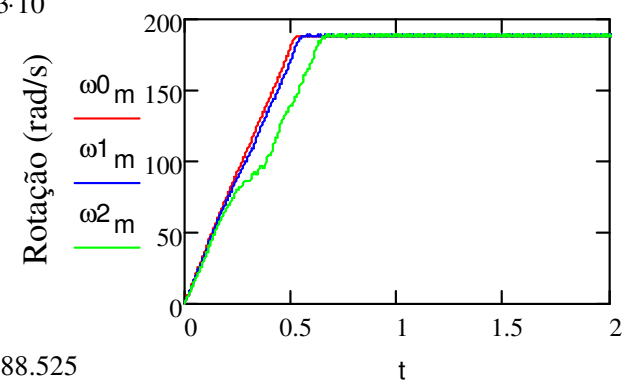

$\max \left(\omega 0_{\mathrm{m}}\right)=188.525$

$\max (\omega 1 \mathrm{~m})=188.831$

$\max (\omega 2 \mathrm{~m})=189.543$

Tempo (s)

Deslocamento x Tempo

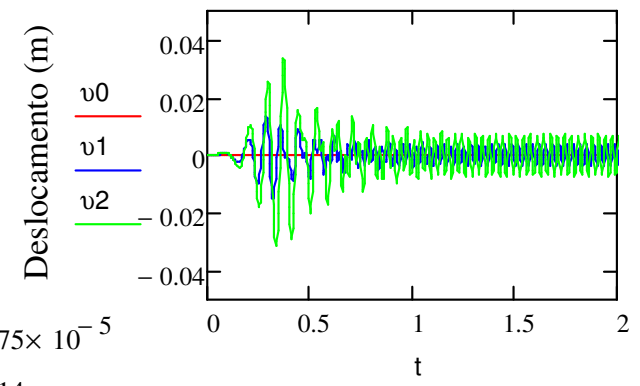

Tempo (s) 
Gráficos D.10 - Respostas da estrutura $b=0.75 \mathrm{~m}, \mathrm{~h}=0.80 \mathrm{~m}$ e $\mathrm{L}=6.0 \mathrm{~m}$

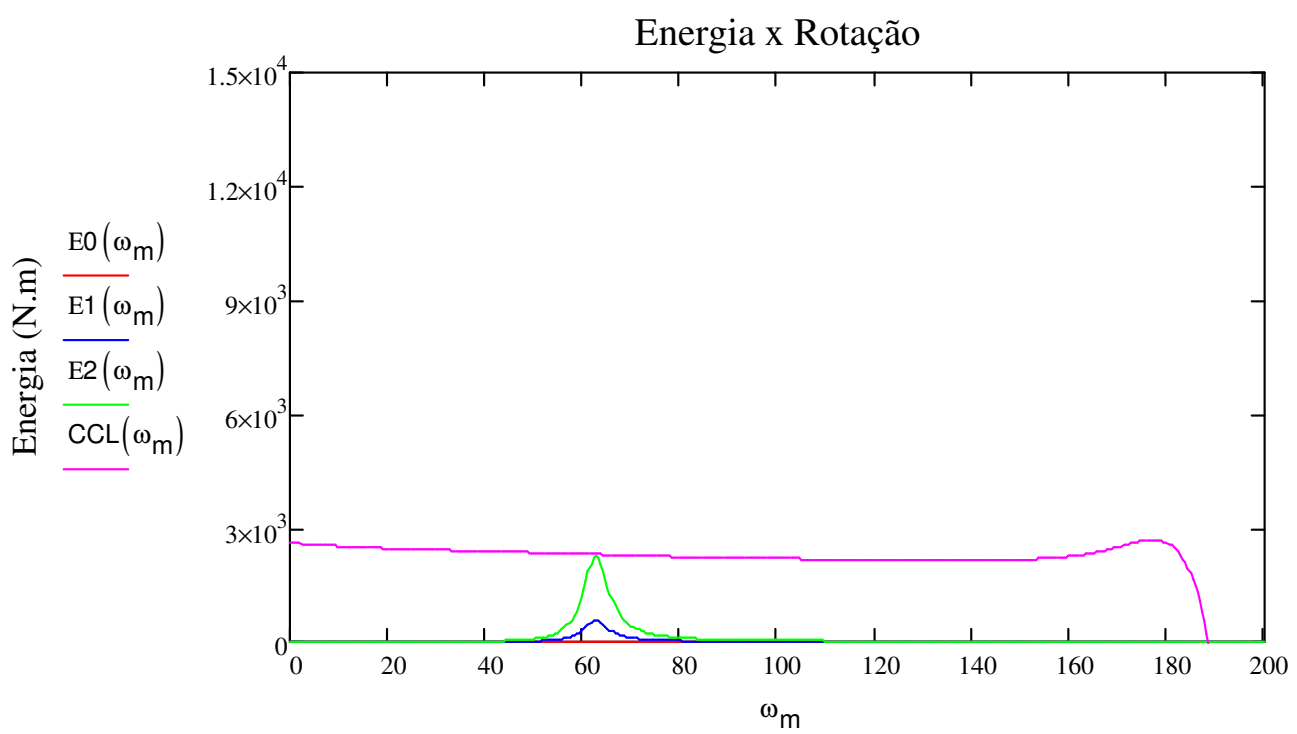

Rotação (rad/s)

— Curva de Energia Consumida pela Estrutura para r0 : $0.034 \mathrm{~mm}$

Curva de Energia Consumida pela Estrutura para r1: $10.67 \mathrm{~mm}$

Curva de Energia Consumida pela Estrutura para r2: $21.34 \mathrm{~mm}$

Curva Característica Líquida do Motor (L-H)

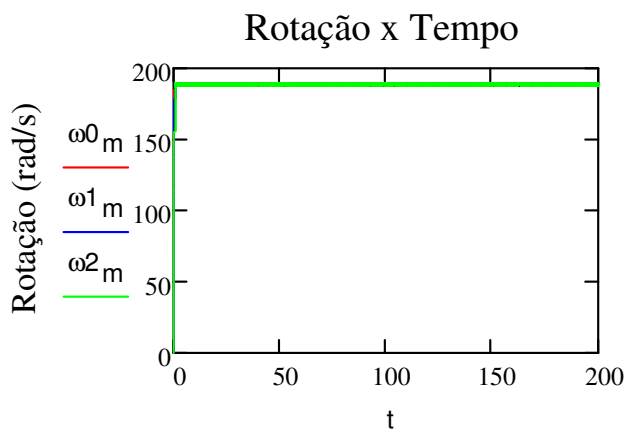

Tempo (s)

Deslocamento x Tempo

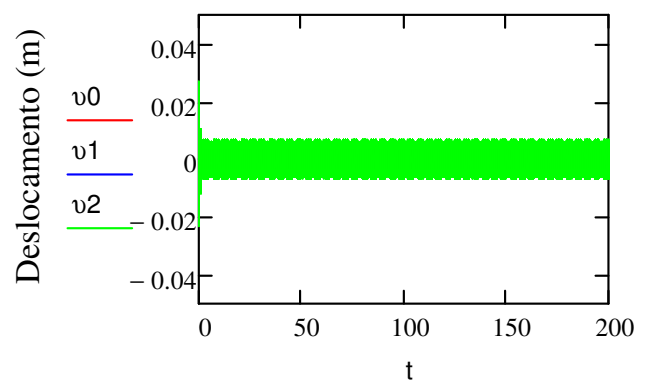

Tempo (s)

$$
\mathrm{E}_{\text {max }}:=5.61 \cdot 10^{-3}
$$

$$
\mathrm{E}_{\text {max }}:=565.08
$$$$
\mathrm{E} 2 \text { max }:=2.26 \cdot 10^{3}
$$

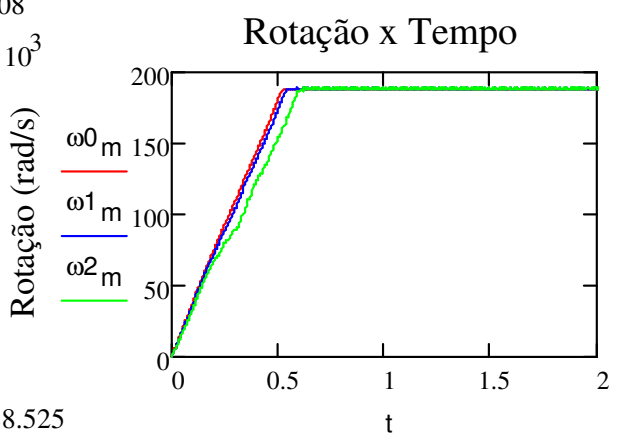

$\max \left(\omega 0_{\mathrm{m}}\right)=188.525$

$\max \left(\omega 1_{\mathrm{m}}\right)=188.791$

$\max \left(\omega 2_{\mathrm{m}}\right)=189.452$

Tempo (s)

Deslocamento x Tempo

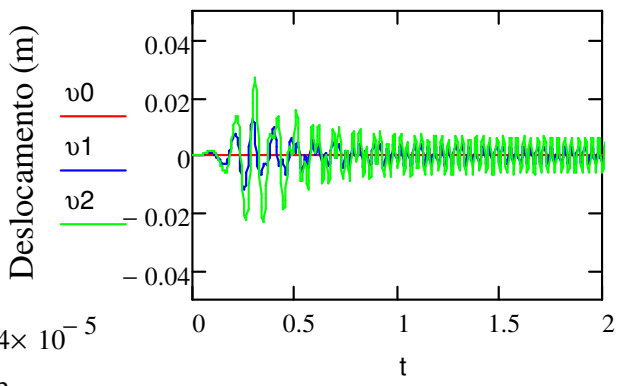

Tempo (s) 
Gráficos D.11 - Respostas da estrutura $b=0.75 \mathrm{~m}, \mathrm{~h}=1.00 \mathrm{~m}$ e $\mathrm{L}=1.5 \mathrm{~m}$

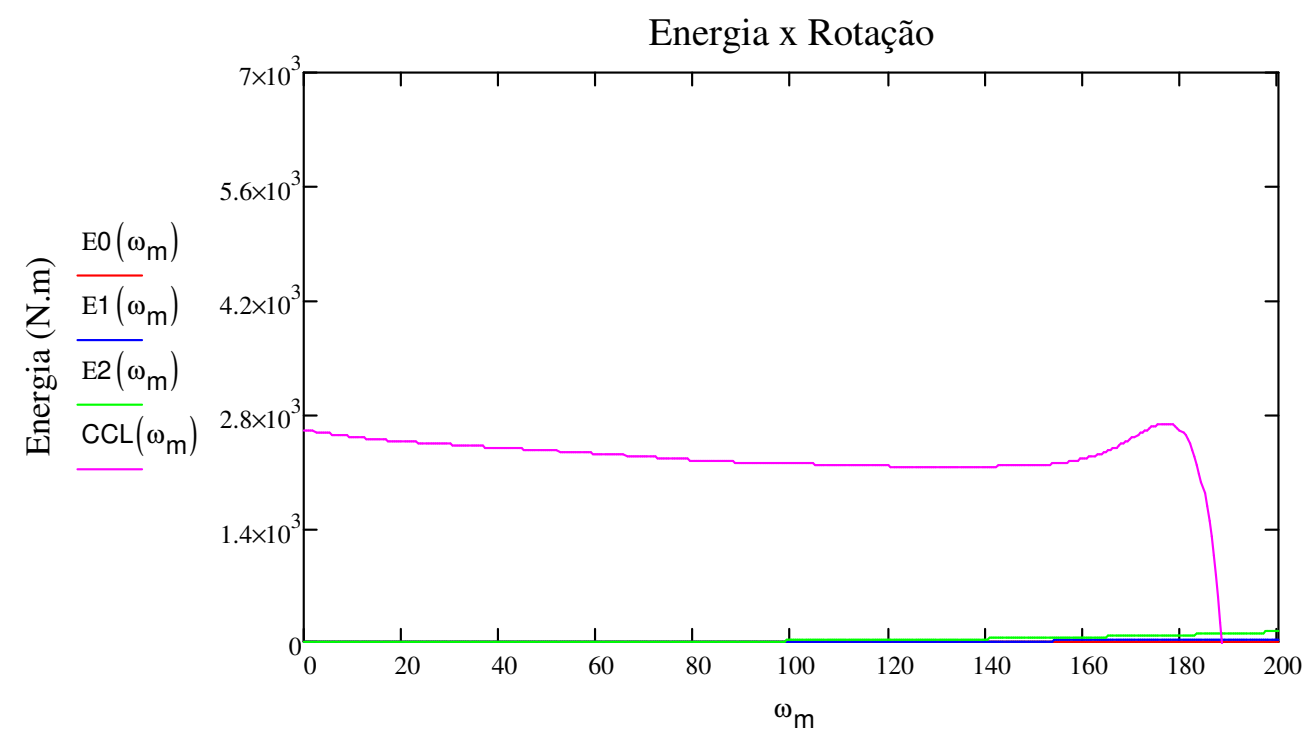

Rotação (rad/s)

— Curva de Energia Consumida pela Estrutura para r0 : $0.034 \mathrm{~mm}$ Curva de Energia Consumida pela Estrutura para r1 : $10.67 \mathrm{~mm}$ Curva de Energia Consumida pela Estrutura para r2: $21.34 \mathrm{~mm}$ Curva Característica Líquida do Motor $(\mathrm{L}-\mathrm{H})$

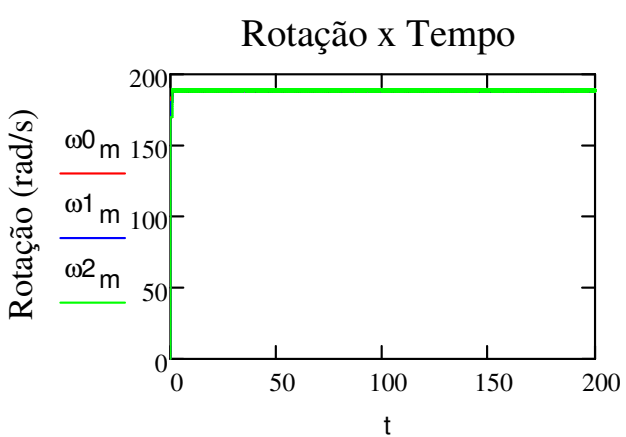

Tempo (s)

Deslocamento x Tempo

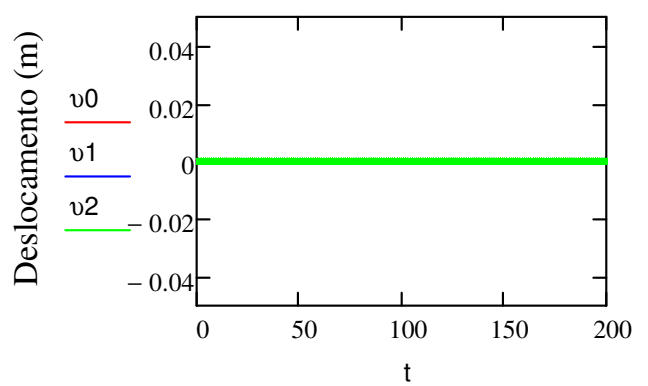

Tempo (s)

$$
\mathrm{E} 0 \text { max }:=3.09 \cdot 10^{-4}
$$$$
\mathrm{E}_{\text {max }}:=31.14
$$$$
\mathrm{E}_{\text {max }} \text { := } 124.55
$$

Rotação x Tempo

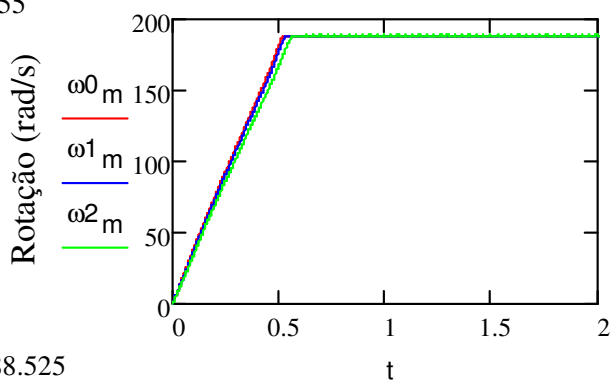

$\max \left(\omega 0_{\mathrm{m}}\right)=188.525$

$\max \left(\omega 1^{\mathrm{m}}\right)=188.591$

$\max \left(\omega 2_{m}\right)=188.676$

Tempo (s)

Deslocamento x Tempo

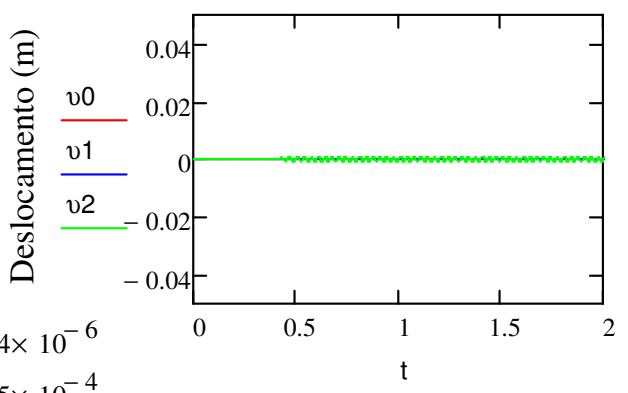

Tempo (s) $\max (v 1)=3.475 \times 10^{-4}$

$\max (v 2)=6.959 \times 10^{-4}$ 
Gráficos D.12 - Respostas da estrutura $b=0.75 \mathrm{~m}, \mathrm{~h}=1.00 \mathrm{~m}$ e $\mathrm{L}=2.0 \mathrm{~m}$

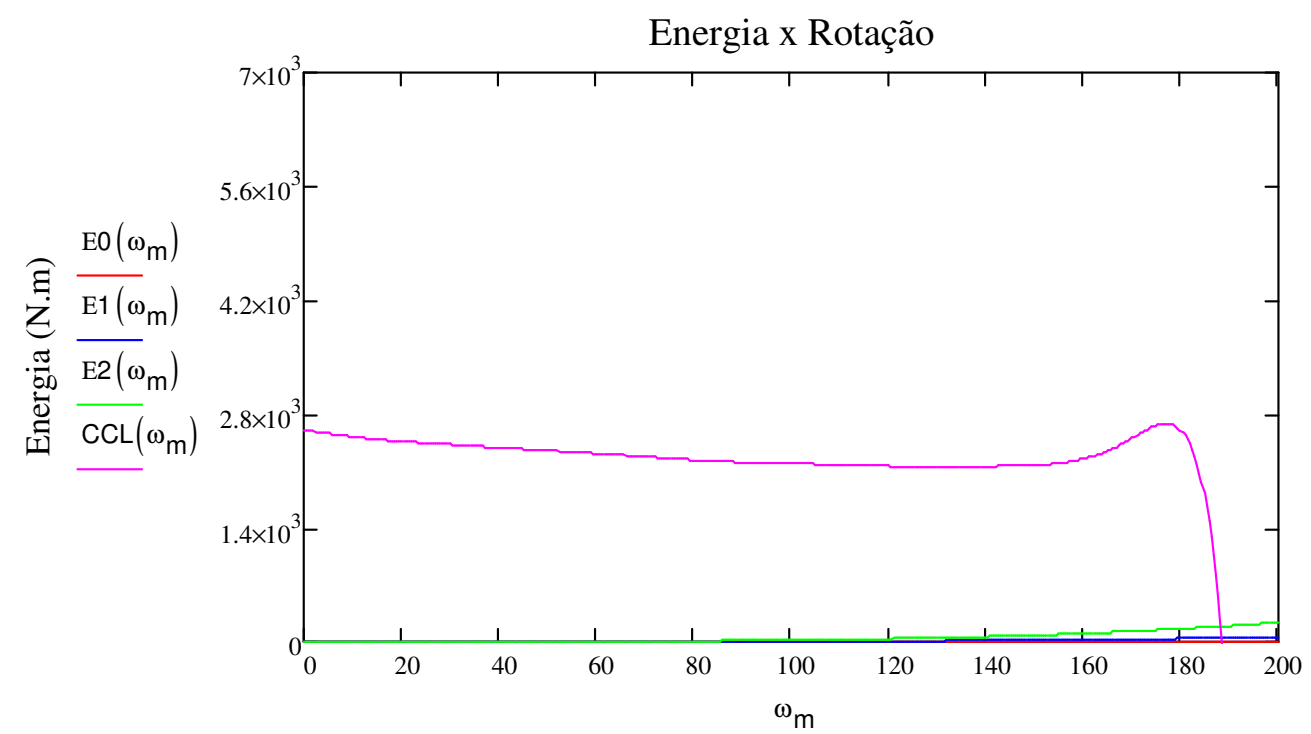

Rotação (rad/s)

— Curva de Energia Consumida pela Estrutura para r0 : $0.034 \mathrm{~mm}$

— Curva de Energia Consumida pela Estrutura para r1 : $10.67 \mathrm{~mm}$

Curva de Energia Consumida pela Estrutura para r2: $21.34 \mathrm{~mm}$

Curva Característica Líquida do Motor $(\mathrm{L}-\mathrm{H})$

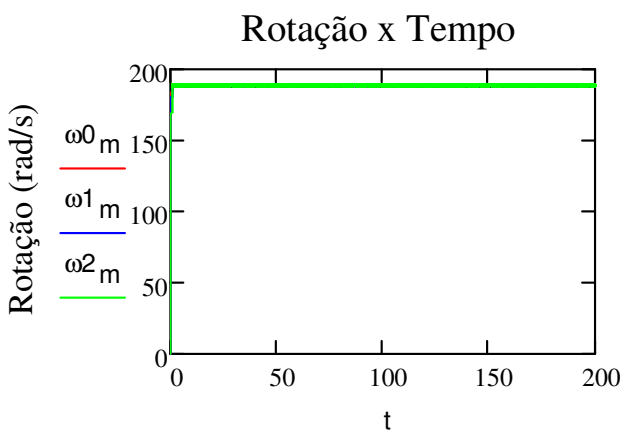

Tempo (s)

Deslocamento x Tempo

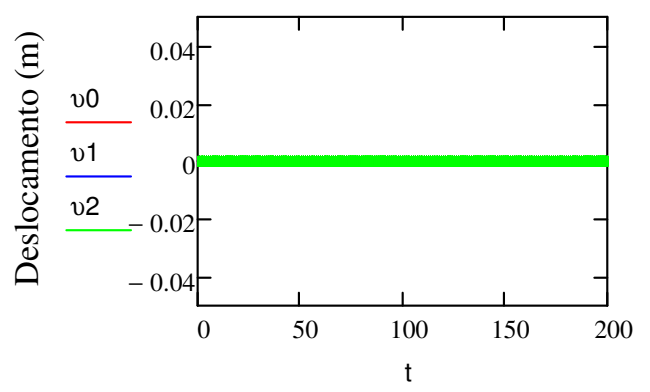

Tempo (s)

$$
\mathrm{E}_{\text {max }}:=6.00 \cdot 10^{-4}
$$$$
\mathrm{E}_{\text {max }}:=60.52
$$$$
\mathrm{E}_{\text {max }}:=242.075
$$

Rotação x Tempo

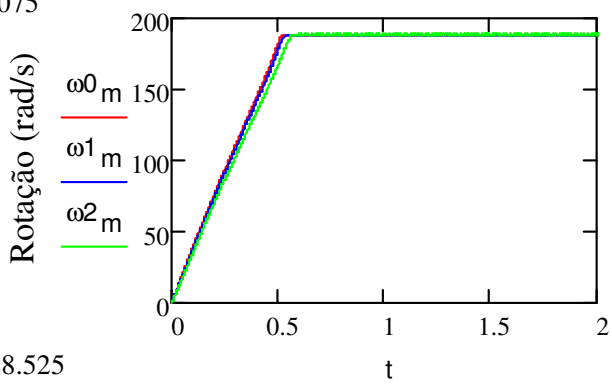

$\max \left(\omega 0_{\mathrm{m}}\right)=188.525$

$\max (\omega 1 \mathrm{~m})=188.613$

$\max \left(\omega 2_{m}\right)=188.782$

Tempo (s)

Deslocamento x Tempo

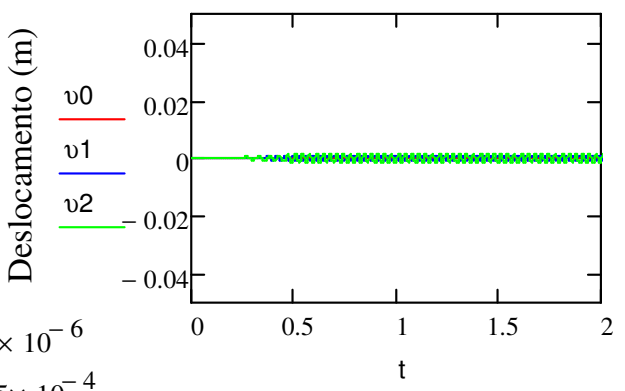

Tempo (s) $\max (v 1)=9.285 \times 10^{-4}$

$\max (v 2)=1.85 \times 10^{-3}$ 
Gráficos D.13 - Respostas da estrutura $b=0.75 \mathrm{~m}, \mathrm{~h}=1.00 \mathrm{~m}$ e $\mathrm{L}=2.5 \mathrm{~m}$

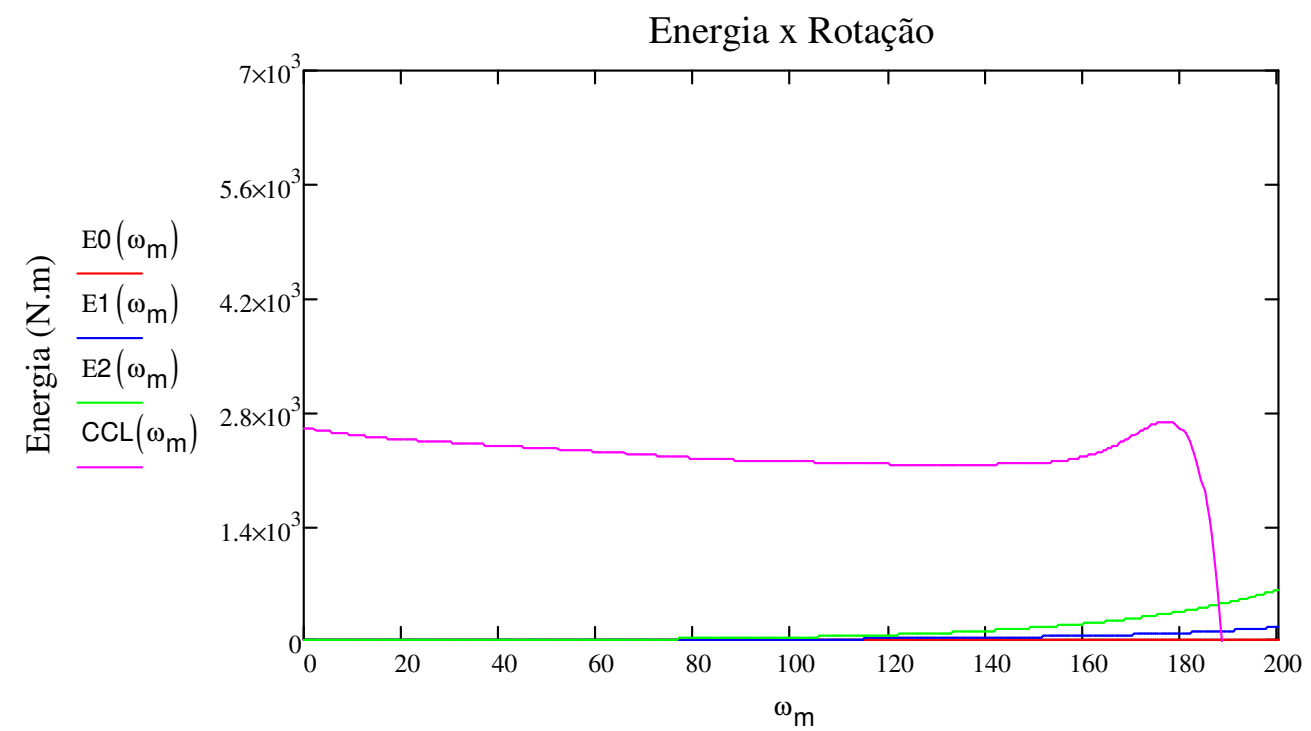

Rotação (rad/s)

— Curva de Energia Consumida pela Estrutura para r0 : $0.034 \mathrm{~mm}$

_ Curva de Energia Consumida pela Estrutura para r1 : $10.67 \mathrm{~mm}$

Curva de Energia Consumida pela Estrutura para r2: $21.34 \mathrm{~mm}$

Curva Característica Líquida do Motor (L-H)

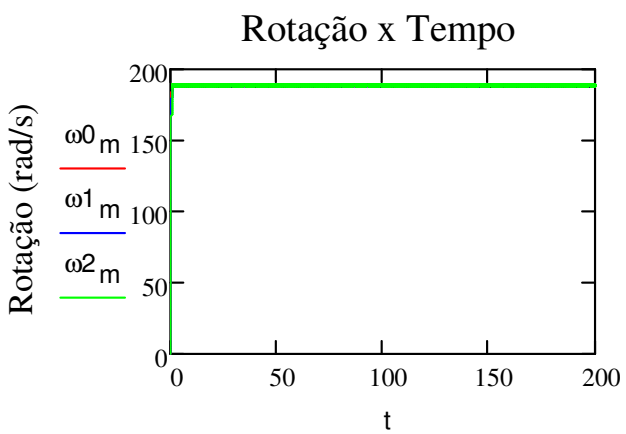

Tempo (s)

Deslocamento x Tempo

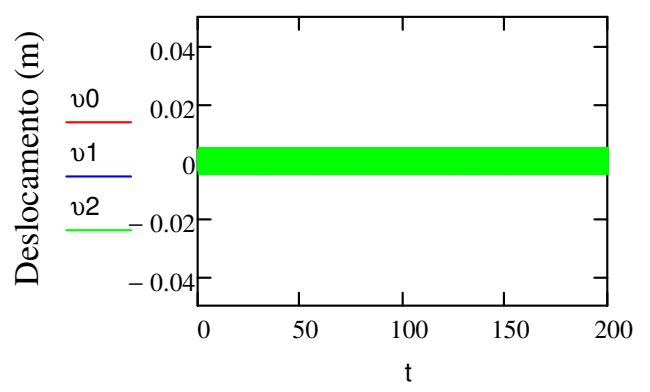

Tempo (s)

$$
\mathrm{E} 0_{\max }:=1.54 \cdot 10^{-3}
$$

$$
\mathrm{E} 1_{\text {max }}:=154.875
$$$$
\mathrm{E}_{\text {max }}:=619.50
$$

Rotação x Tempo

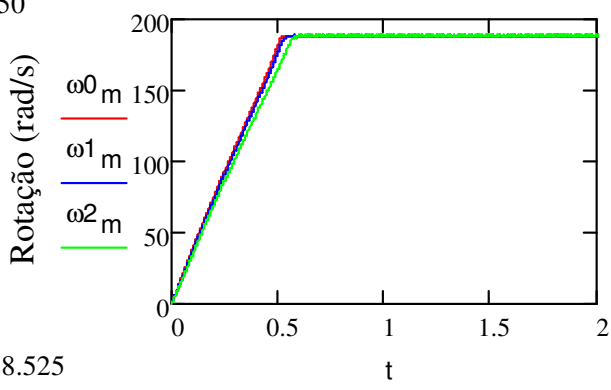

$\max \left(\omega 0_{\mathrm{m}}\right)=188.525$

$\max (\omega 1 \mathrm{~m})=188.665$

$\max \left(\omega 2_{m}\right)=188.968$

Tempo (s)

Deslocamento x Tempo

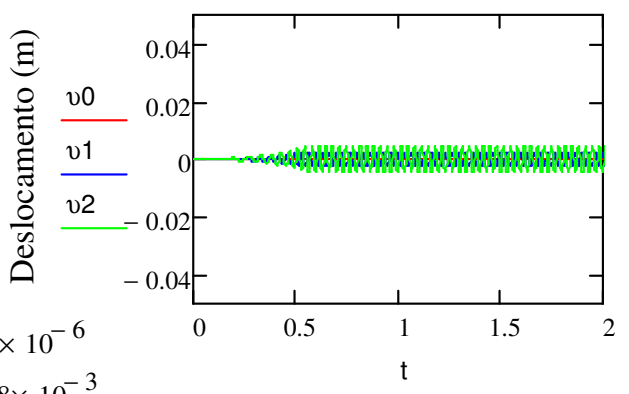

Tempo (s) $\max (v 1)=2.388 \times 10^{-3}$

$\max (v 2)=4.753 \times 10^{-3}$ 
Gráficos D.14 - Respostas da estrutura $b=0.75 \mathrm{~m}, \mathrm{~h}=1.00 \mathrm{~m}$ e $\mathrm{L}=3.0 \mathrm{~m}$

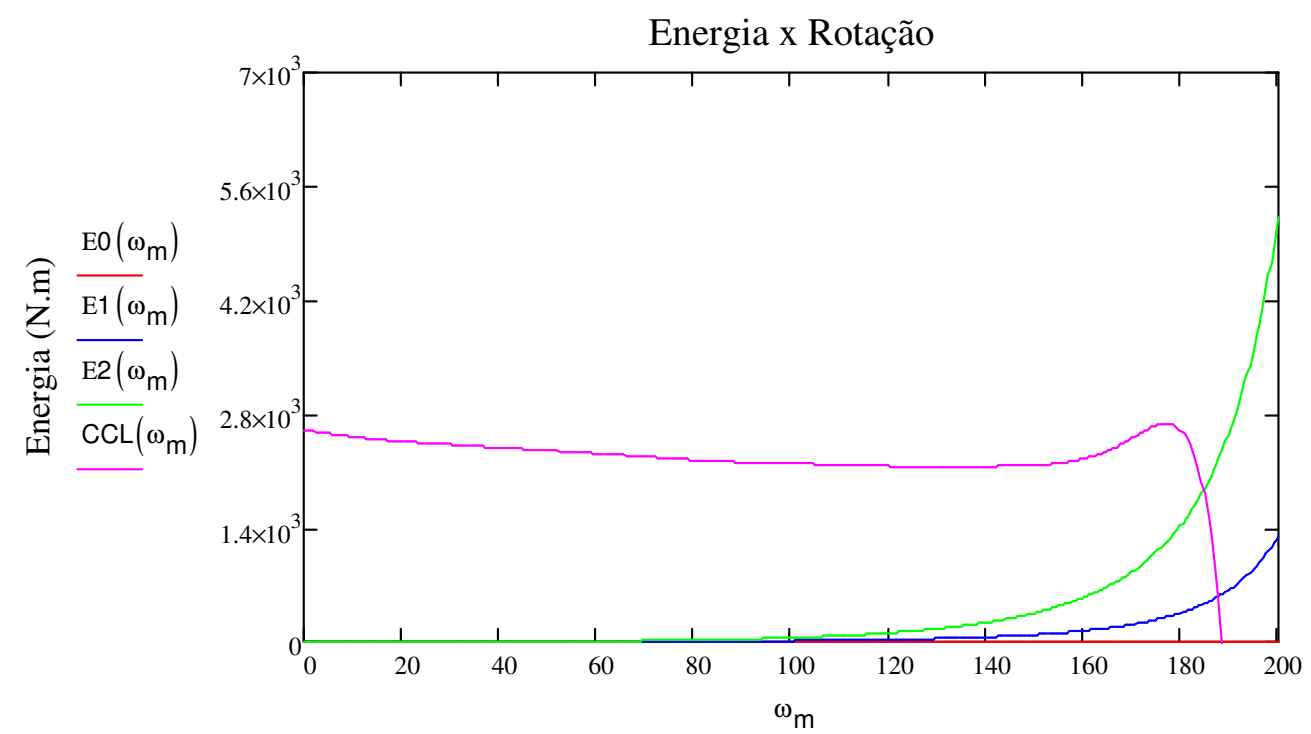

Rotação (rad/s)

— Curva de Energia Consumida pela Estrutura para r0 : $0.034 \mathrm{~mm}$

_ Curva de Energia Consumida pela Estrutura para r1 : $10.67 \mathrm{~mm}$

Curva de Energia Consumida pela Estrutura para r2: $21.34 \mathrm{~mm}$

Curva Característica Líquida do Motor (L-H)

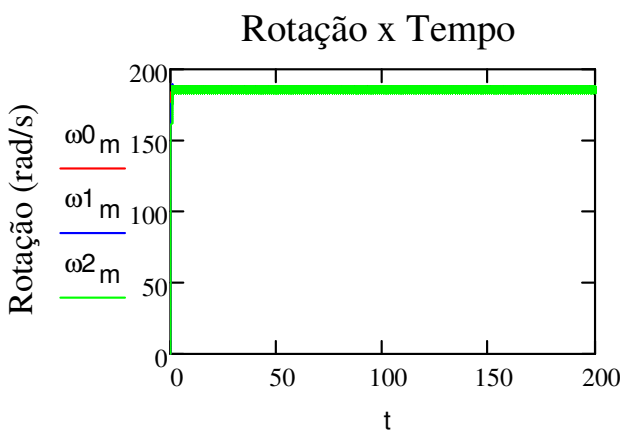

Tempo (s)

Deslocamento x Tempo

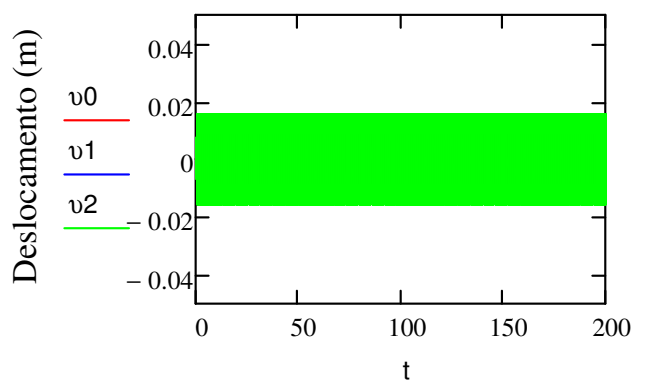

Tempo (s)

$$
\begin{aligned}
& \mathrm{E}_{\text {max }}:=0.013 \\
& \mathrm{E}_{\text {max }}:=1.31 \cdot 10^{3} \\
& \mathrm{E}_{\text {max }}:=5.23 \cdot 10^{3}
\end{aligned}
$$

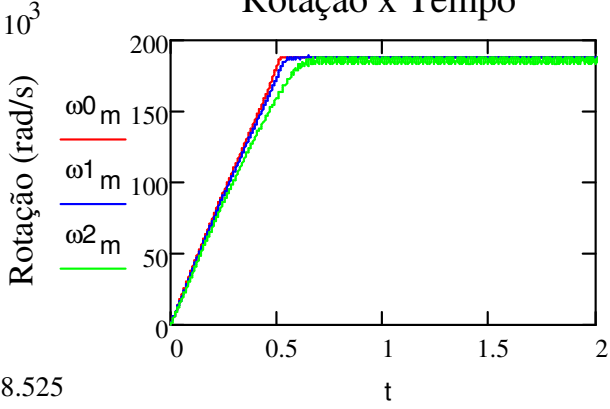

$\max \left(\omega 0_{\mathrm{m}}\right)=188.525$

$\max \left(\omega 1_{\mathrm{m}}\right)=188.642$

$\max (\omega 2 \mathrm{~m})=187.931$

Tempo (s)

Deslocamento x Tempo

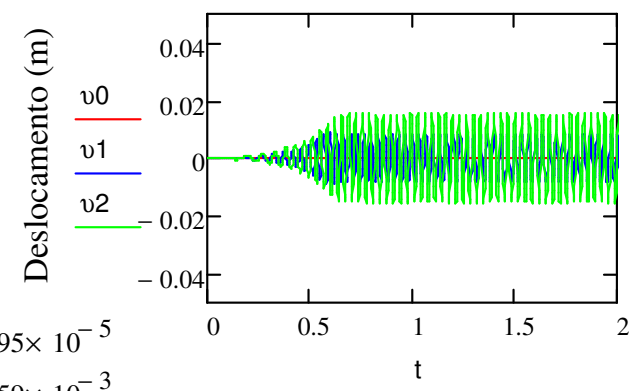

Tempo (s) $\max (v 0)=2.895 \times 10^{-5}$

$\max (v 1)=8.859 \times 10^{-3}$

$\max (v 2)=0.015$ 
Gráficos D.15 - Respostas da estrutura $b=0.75 \mathrm{~m}, \mathrm{~h}=1.00 \mathrm{~m}$ e $\mathrm{L}=3.5 \mathrm{~m}$

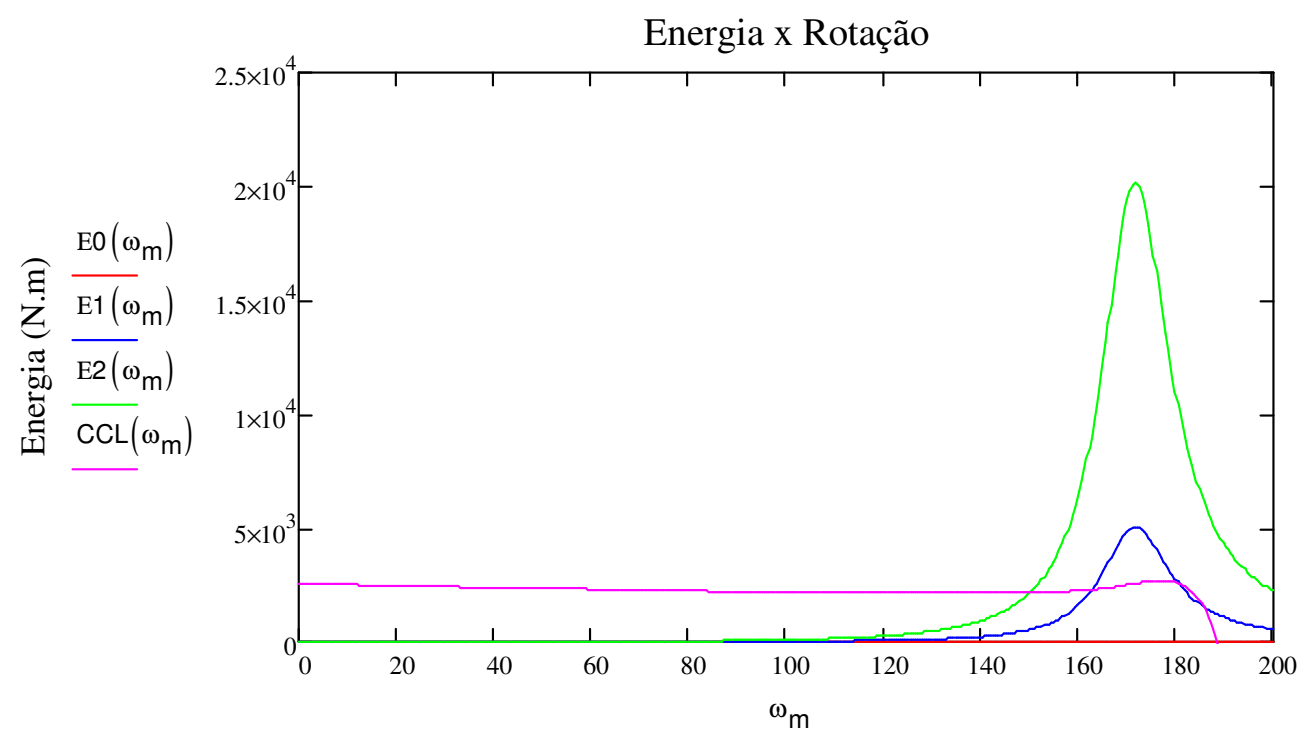

Rotação (rad/s)

— Curva de Energia Consumida pela Estrutura para r0 : $0.034 \mathrm{~mm}$

— Curva de Energia Consumida pela Estrutura para r1 : $10.67 \mathrm{~mm}$

Curva de Energia Consumida pela Estrutura para r2: $21.34 \mathrm{~mm}$ Curva Característica Líquida do Motor (L-H)

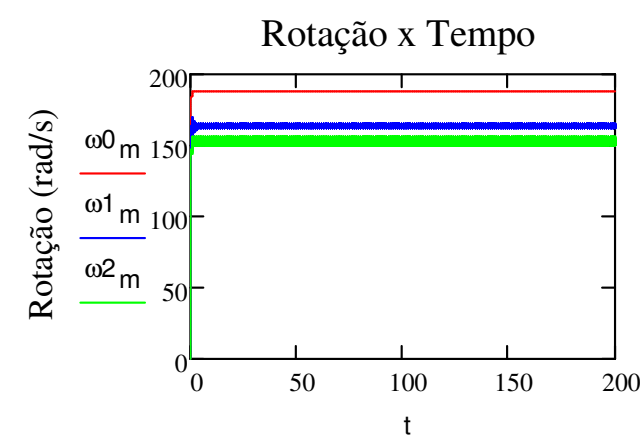

Tempo (s)

Deslocamento x Tempo

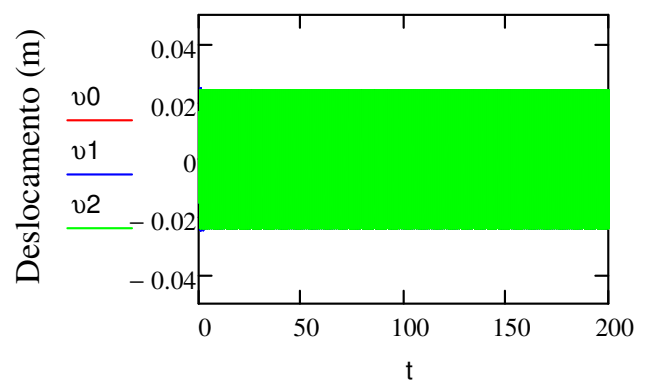

Tempo (s)

$$
\begin{aligned}
& \mathrm{E}_{\text {max }}:=0.05 \\
& \mathrm{E} 1_{\text {max }}:=5.03610^{3} \\
& \mathrm{E}_{\text {max }}:=2.01410^{4}
\end{aligned}
$$

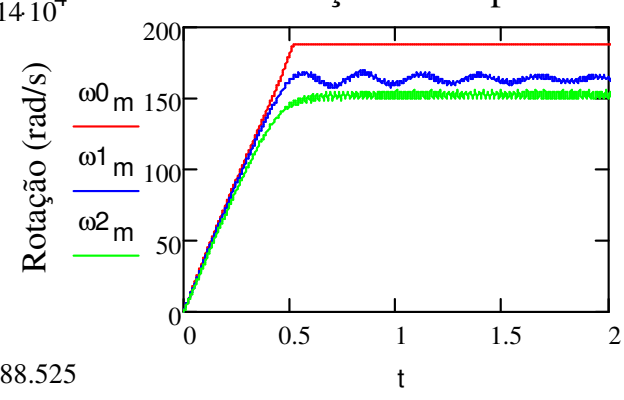

$\max \left(\omega 0_{\mathrm{m}}\right)=188.525$

$\max \left(\omega 1_{1}\right)=169.081$

$\max (\omega 2 \mathrm{~m})=155.38$

Tempo (s)

Deslocamento x Tempo

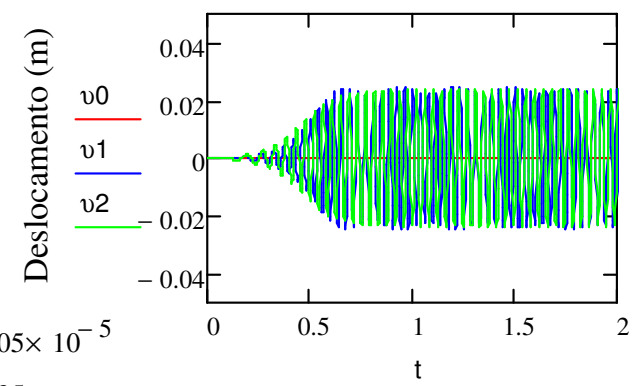

Tempo (s) 
Gráficos D.16 - Respostas da estrutura $b=0.75 \mathrm{~m}, \mathrm{~h}=1.00 \mathrm{~m}$ e $\mathrm{L}=4.0 \mathrm{~m}$

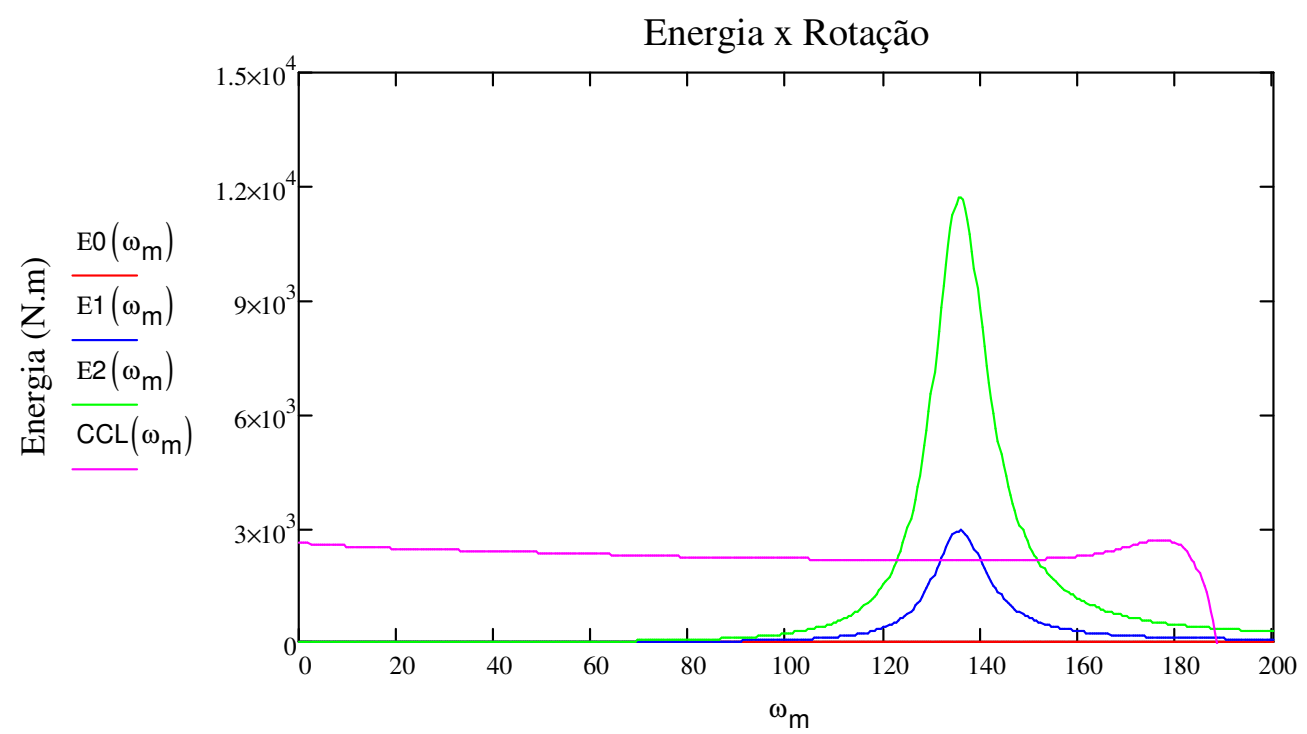

Rotação (rad/s)

— Curva de Energia Consumida pela Estrutura para r0 : $0.034 \mathrm{~mm}$

_ Curva de Energia Consumida pela Estrutura para r1 : $10.67 \mathrm{~mm}$

Curva de Energia Consumida pela Estrutura para r2: $21.34 \mathrm{~mm}$ Curva Característica Líquida do Motor (L-H)

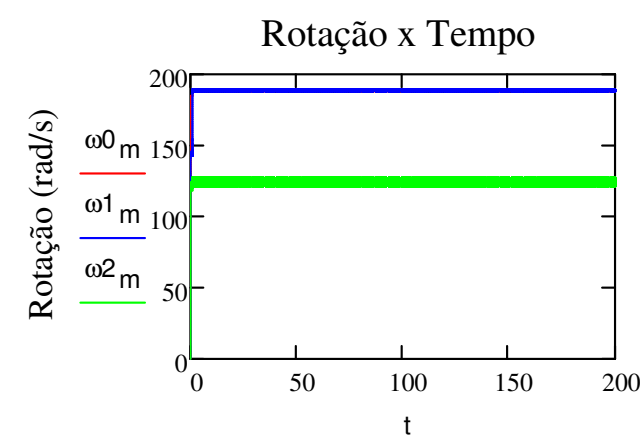

Tempo (s)

Deslocamento x Tempo

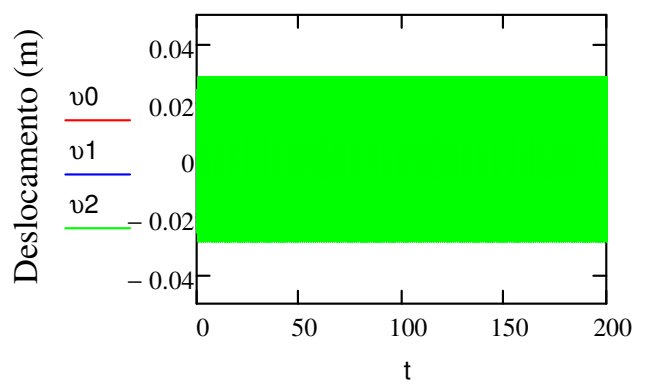

Tempo (s)

$$
\begin{aligned}
& \mathrm{E}_{\text {max }}:=0.029 \\
& \mathrm{E} 1_{\text {max }}:=2.93 \cdot 10^{3} \\
& \mathrm{E}_{\text {max }}:=1.172 \cdot 10^{4}
\end{aligned}
$$

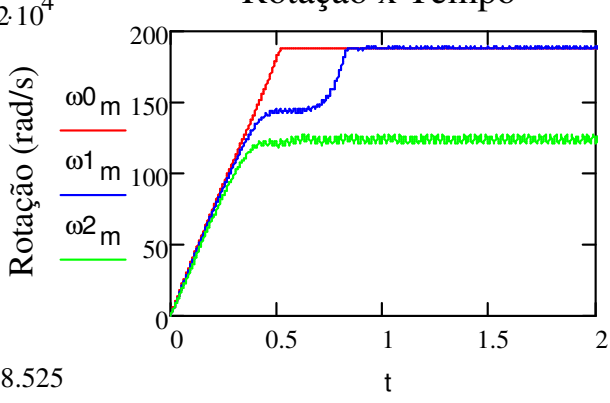

$\max \left(\omega 0_{\mathrm{m}}\right)=188.525$

$\max (\omega 1 \mathrm{~m})=189.087$

$\max (\omega 2 \mathrm{~m})=127.13$

Tempo (s)

Deslocamento x Tempo

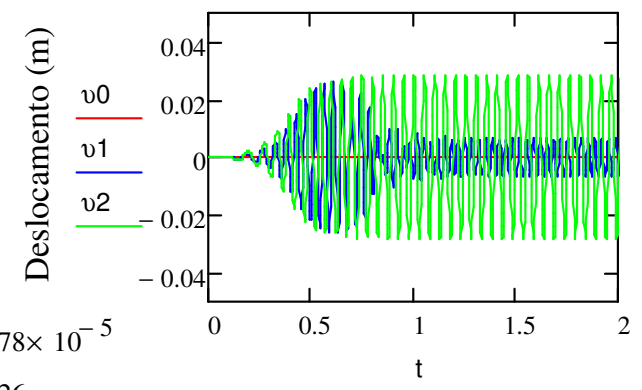

Tempo (s) 
Gráficos D.17 - Respostas da estrutura $b=0.75 \mathrm{~m}, \mathrm{~h}=1.00 \mathrm{~m}$ e $\mathrm{L}=4.5 \mathrm{~m}$

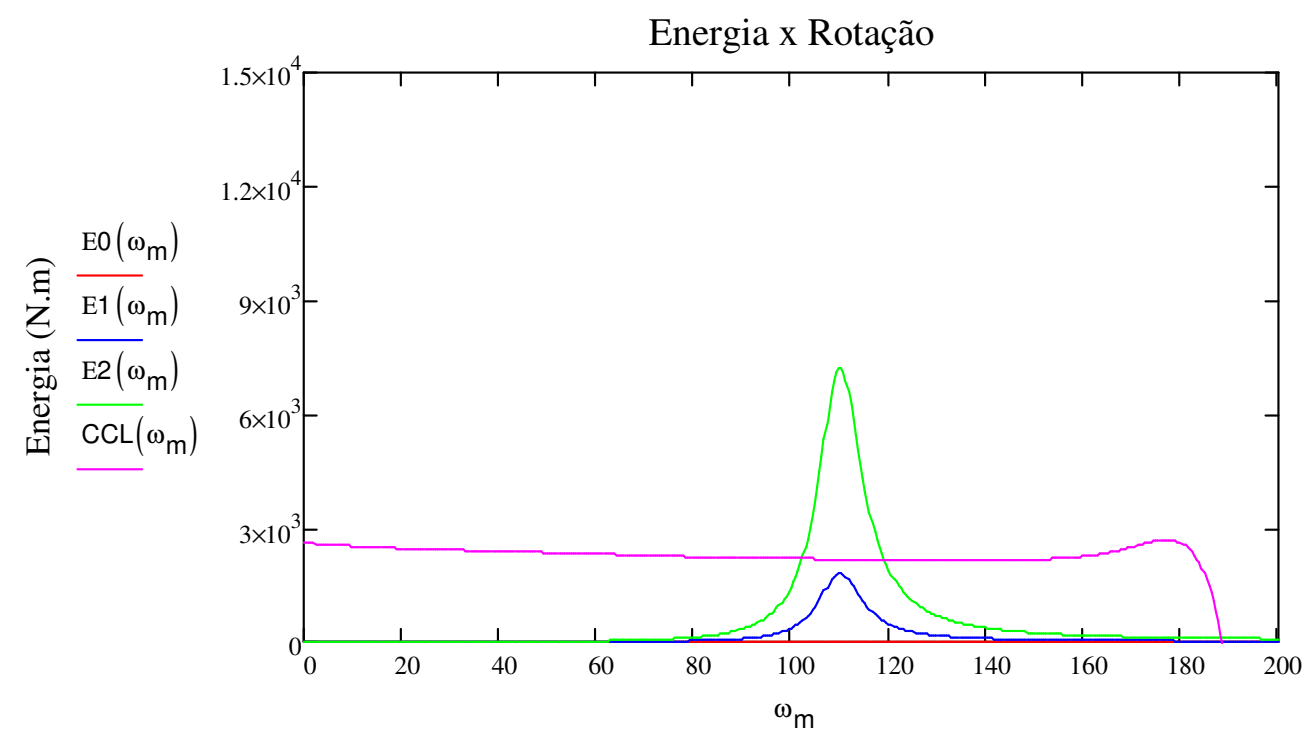

Rotação (rad/s)

_ Curva de Energia Consumida pela Estrutura para r0 : $0.034 \mathrm{~mm}$

— Curva de Energia Consumida pela Estrutura para r1 : $10.67 \mathrm{~mm}$

Curva de Energia Consumida pela Estrutura para r2: $21.34 \mathrm{~mm}$ Curva Característica Líquida do Motor (L-H)

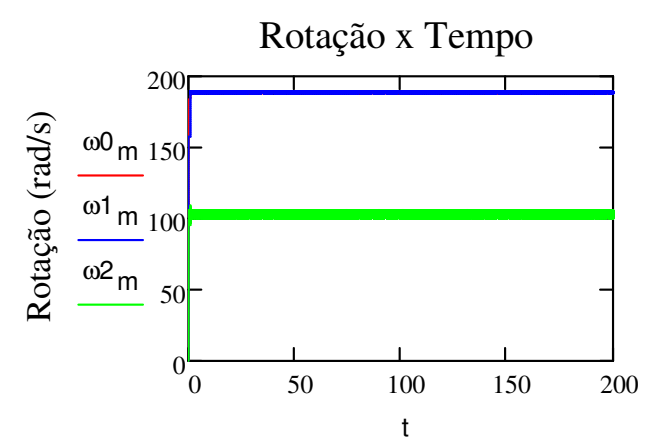

Tempo (s)

Deslocamento x Tempo

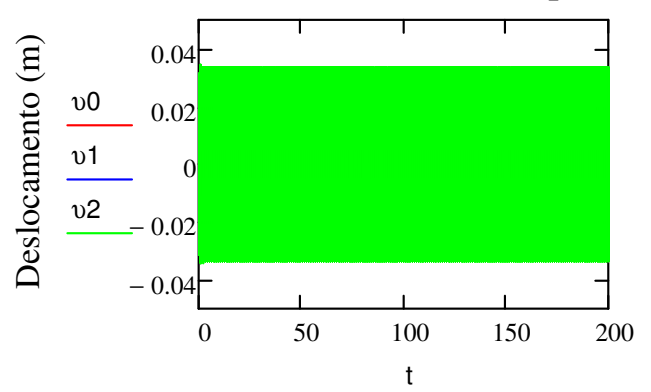

Tempo (s)

$$
\begin{aligned}
& \mathrm{E}_{\text {max }}:=0.018 \\
& \mathrm{E}_{\text {max }}:=1.802 \cdot 10^{3} \\
& \mathrm{E}_{\text {max }}:=7.208 \cdot 10^{3}
\end{aligned}
$$

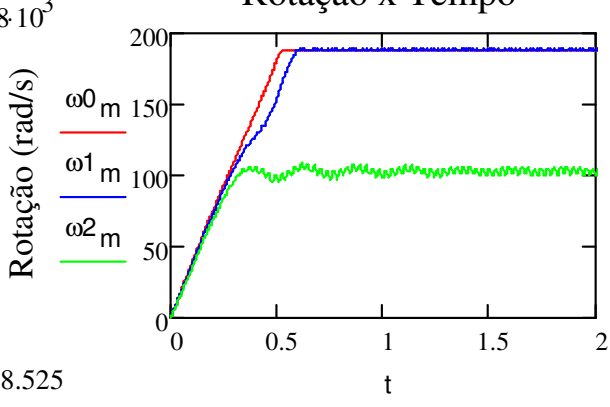

$\max \left(\omega 0_{m}\right)=188.525$

$\max (\omega 1 \mathrm{~m})=188.907$

$\max \left(\omega 2_{m}\right)=108.448$

Tempo (s)

Deslocamento x Tempo

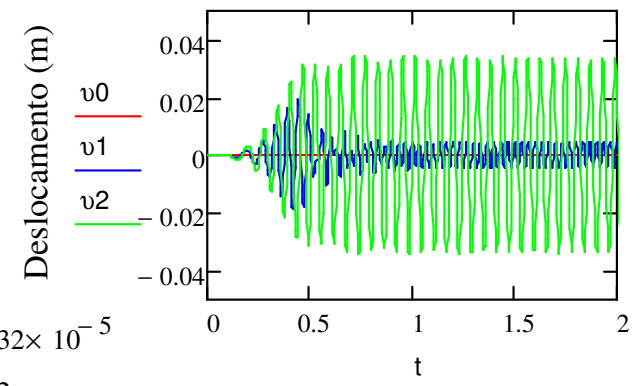

Tempo (s) 
Gráficos D.18 - Respostas da estrutura $b=0.75 \mathrm{~m}, \mathrm{~h}=1.00 \mathrm{~m}$ e $\mathrm{L}=5.0 \mathrm{~m}$

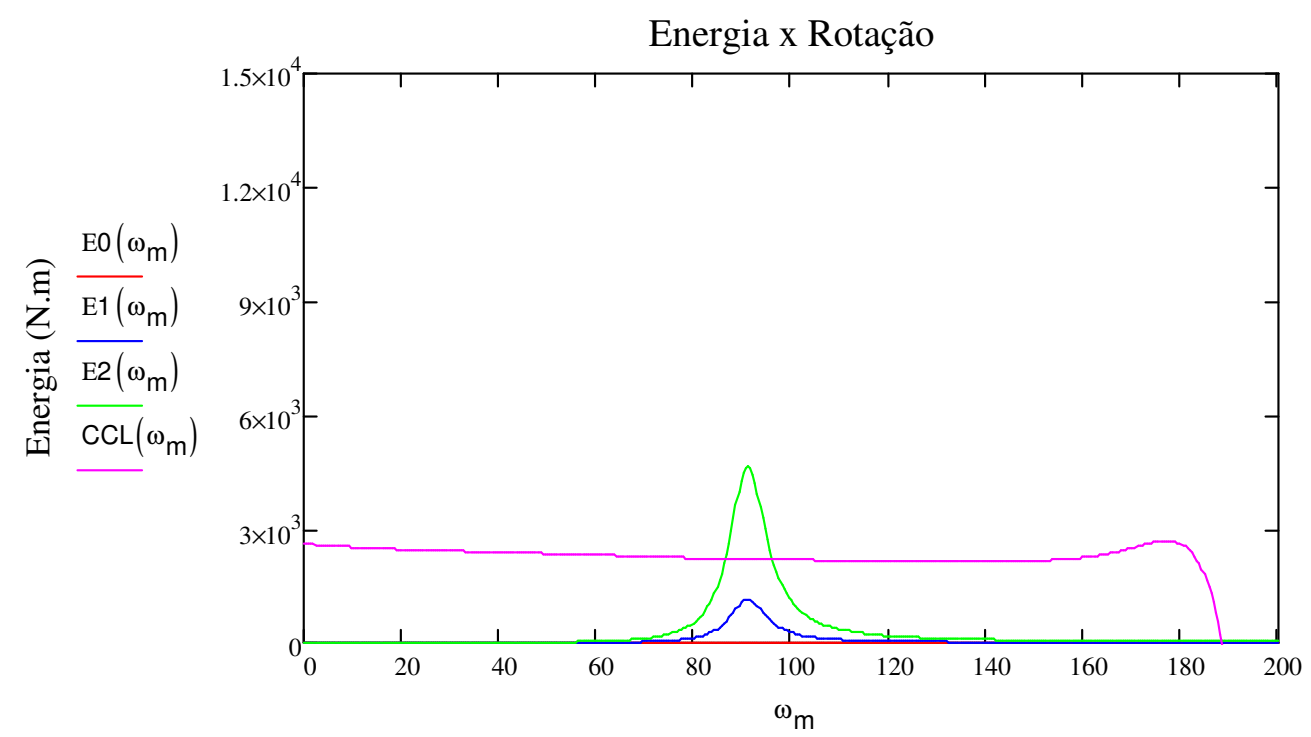

Rotação (rad/s)

_ Curva de Energia Consumida pela Estrutura para r0 : $0.034 \mathrm{~mm}$

_ Curva de Energia Consumida pela Estrutura para r1 : $10.67 \mathrm{~mm}$

Curva de Energia Consumida pela Estrutura para r2: $21.34 \mathrm{~mm}$ Curva Característica Líquida do Motor (L-H)

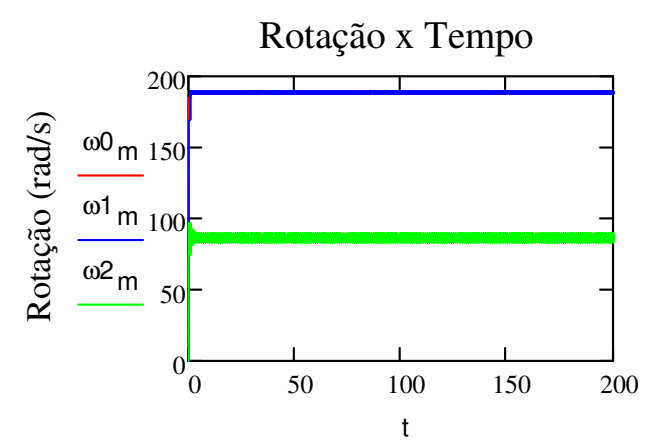

Tempo (s)

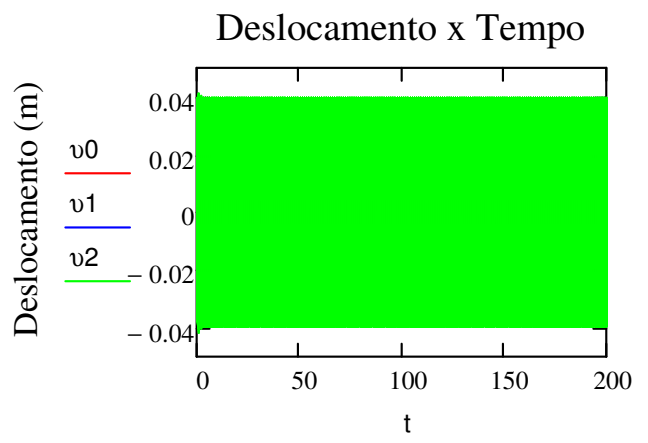

Tempo (s)

$$
\begin{aligned}
& \mathrm{E}_{\text {max }}:=0.012 \\
& \mathrm{E}_{\text {max }}:=1.163 \cdot 10^{3} \\
& \mathrm{E}_{\text {max }}:=4.65 \cdot 10^{3}
\end{aligned}
$$

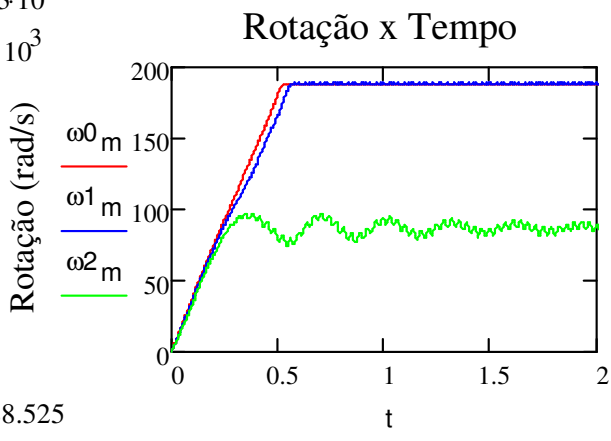

$\max \left(\omega 0_{\mathrm{m}}\right)=188.525$

$\max \left(\omega 1_{\mathrm{m}}\right)=188.828$

$\max (\omega 2 \mathrm{~m})=97.513$

Tempo (s)

Deslocamento x Tempo

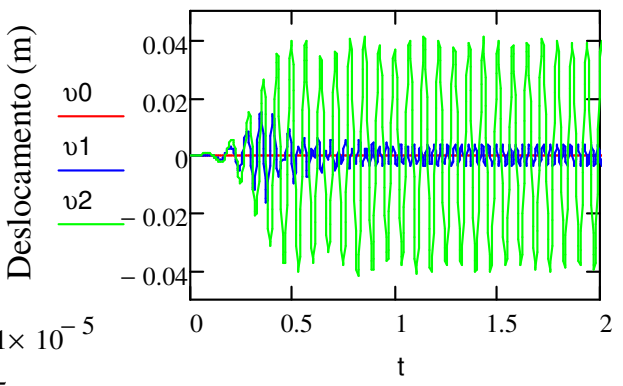

$\max (v 1)=0.015$

$\max (v 2)=0.041$

Tempo (s) 
Gráficos D.19 - Respostas da estrutura $b=0.75 \mathrm{~m}, \mathrm{~h}=1.00 \mathrm{~m}$ e $\mathrm{L}=5.5 \mathrm{~m}$

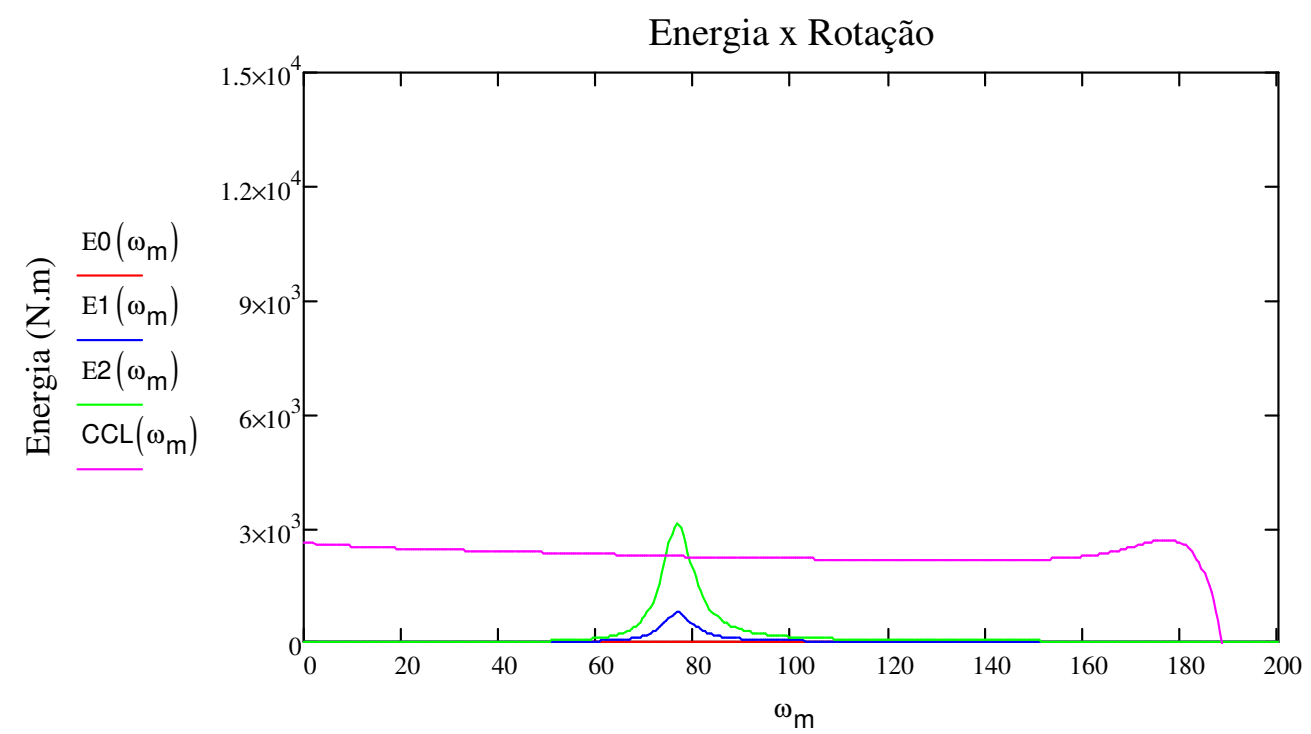

Rotação (rad/s)

— Curva de Energia Consumida pela Estrutura para r0 : $0.034 \mathrm{~mm}$

— Curva de Energia Consumida pela Estrutura para r1: $10.67 \mathrm{~mm}$

Curva de Energia Consumida pela Estrutura para r2: $21.34 \mathrm{~mm}$

Curva Característica Líquida do Motor (L-H)

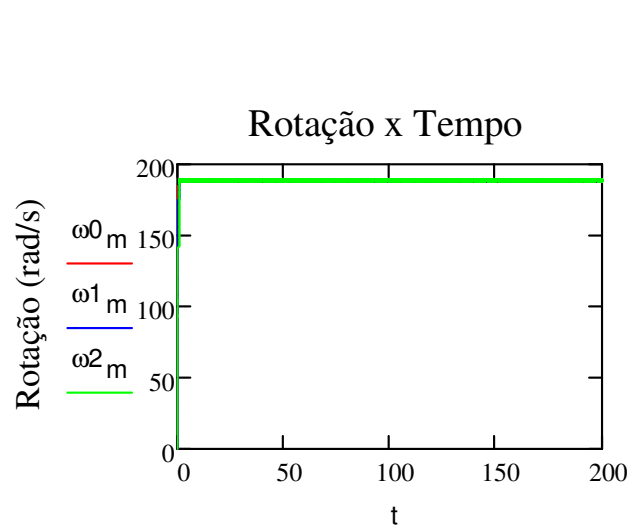

Tempo (s)

Deslocamento x Tempo

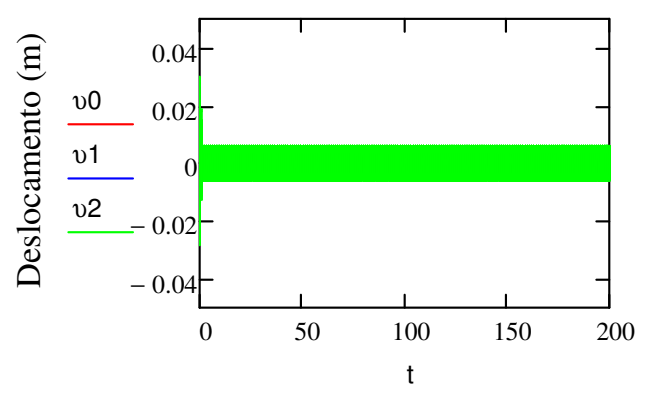

Tempo (s)
$\mathrm{E}_{\max }:=7.70 \cdot 10^{-3}$

$\mathrm{E1}{ }_{\max }:=775.46$

$\mathrm{E} 2 \max :=3.102 \cdot 10^{3}$

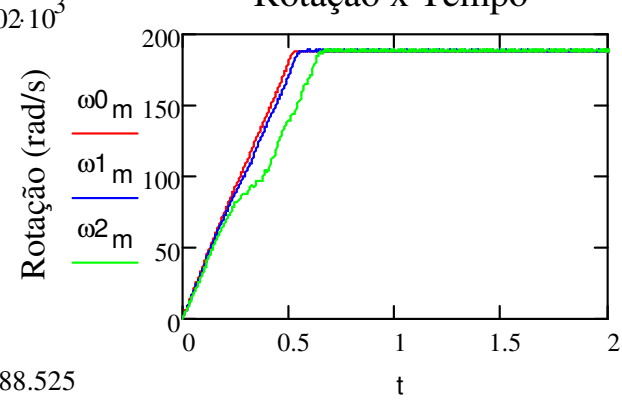

$\max \left(\omega 0_{\mathrm{m}}\right)=188.525$

$\max (\omega 1 \mathrm{~m})=188.825$

$\max \left(\omega 2_{\mathrm{m}}\right)=189.498$

Tempo (s)

Deslocamento x Tempo

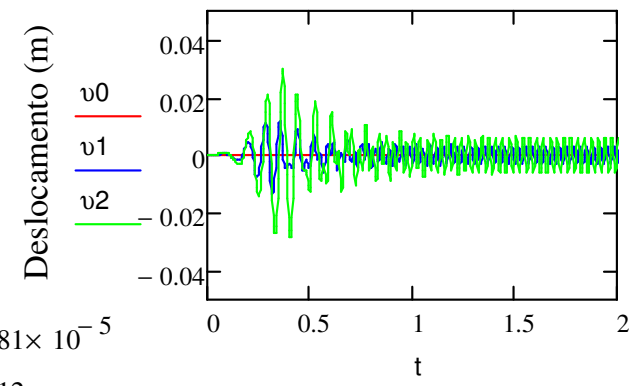

Tempo (s)

$\max (v 1)=0.012$

$\max (v 2)=0.03$ 
Gráficos D.20 - Respostas da estrutura $b=0.75 \mathrm{~m}, \mathrm{~h}=1.00 \mathrm{~m}$ e $\mathrm{L}=6.0 \mathrm{~m}$

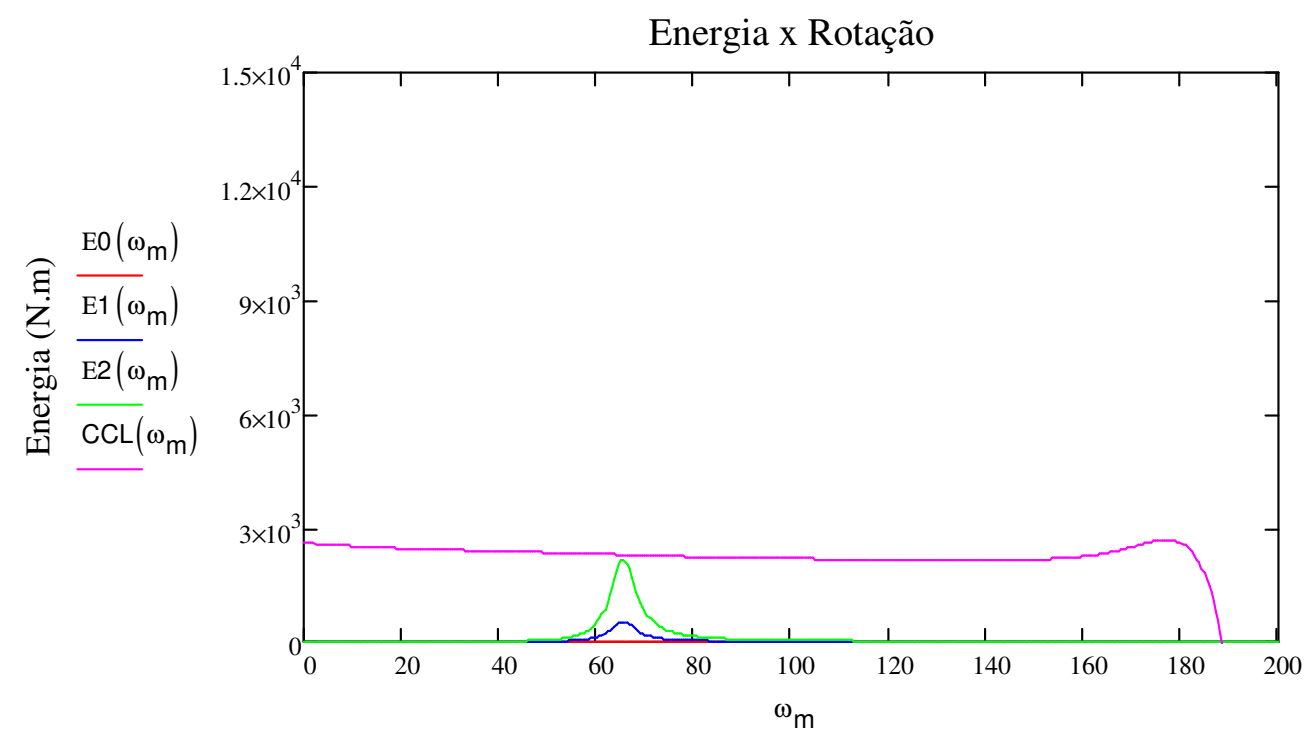

Rotação (rad/s)

— Curva de Energia Consumida pela Estrutura para r0 : $0.034 \mathrm{~mm}$

— Curva de Energia Consumida pela Estrutura para r1: $10.67 \mathrm{~mm}$

Curva de Energia Consumida pela Estrutura para r2: $21.34 \mathrm{~mm}$

Curva Característica Líquida do Motor (L-H)

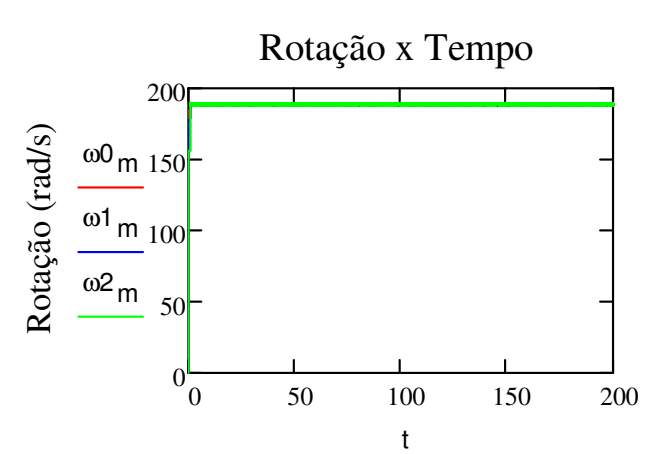

Tempo (s)

Deslocamento x Tempo

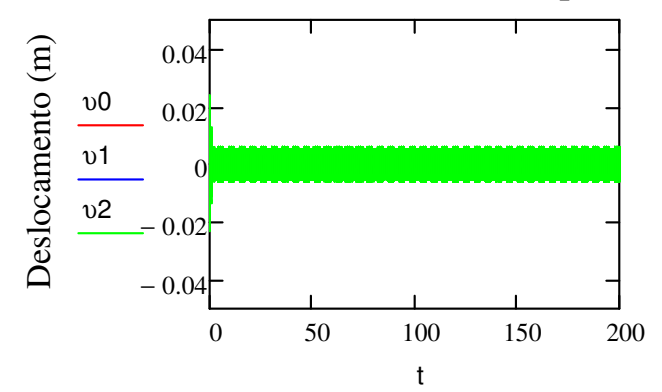

Tempo (s)
$\mathrm{E}_{\text {max }}:=5.305 \cdot 10^{-3}$

$\mathrm{E}_{\text {max }}:=534.645$

$\mathrm{E} 2$ max $:=2.139 \cdot 10^{3}$

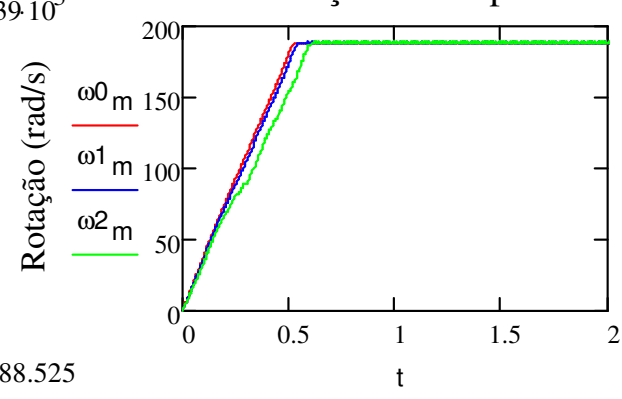

$\max \left(\omega 0_{m}\right)=188.525$

$\max (\omega 1 \mathrm{~m})=188.776$

$\max (\omega 2 \mathrm{~m})=189.28$

Tempo (s)

Deslocamento x Tempo

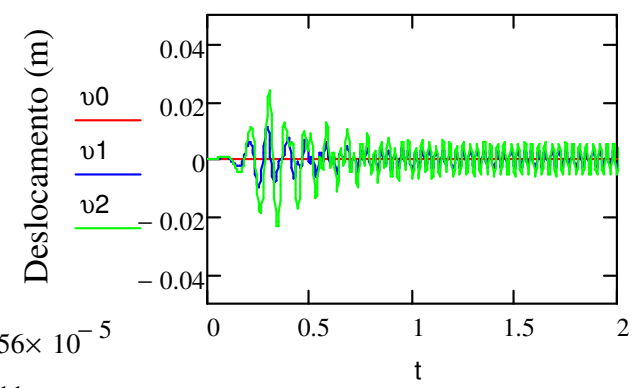

Tempo (s) 
Gráficos D.21 - Respostas da estrutura $b=0.95 \mathrm{~m}, \mathrm{~h}=0.80 \mathrm{~m}$ e $\mathrm{L}=1.5 \mathrm{~m}$

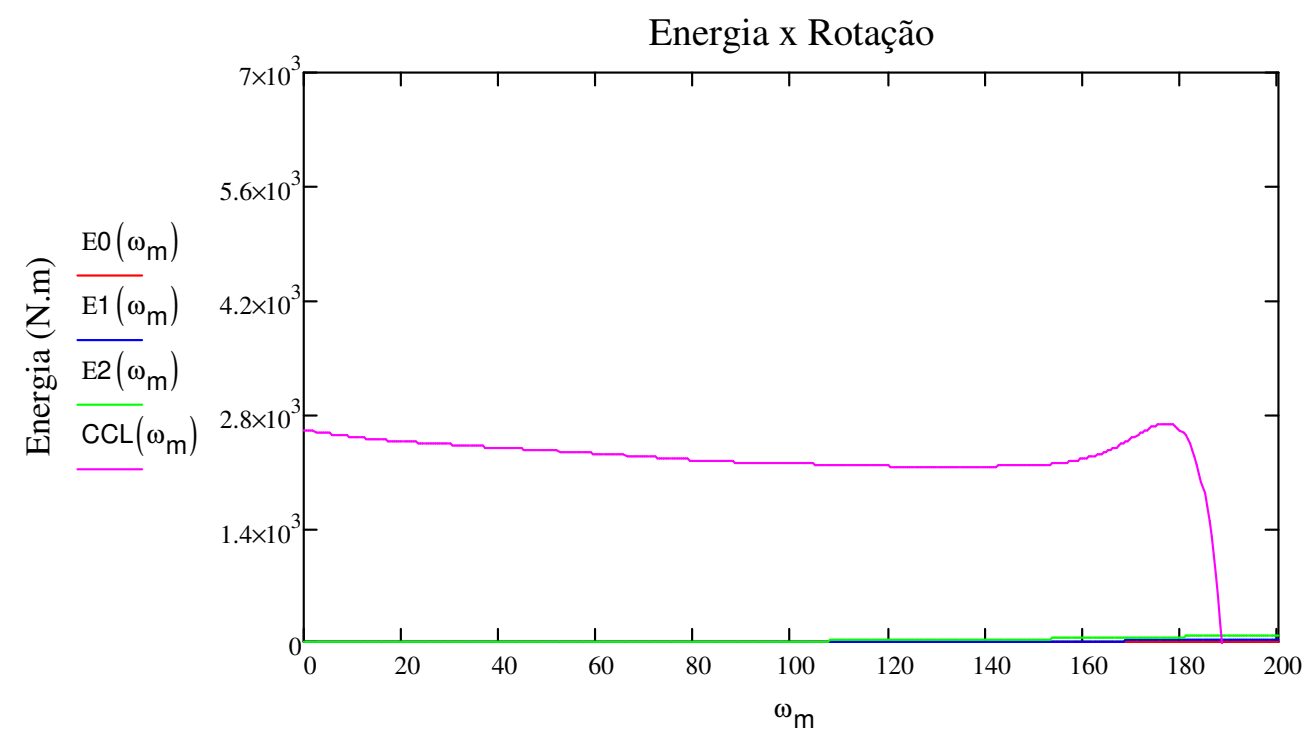

Rotação (rad/s)

— Curva de Energia Consumida pela Estrutura para r0 : $0.034 \mathrm{~mm}$ Curva de Energia Consumida pela Estrutura para r1 : $10.67 \mathrm{~mm}$ Curva de Energia Consumida pela Estrutura para r2: $21.34 \mathrm{~mm}$ Curva Característica Líquida do Motor (L-H)

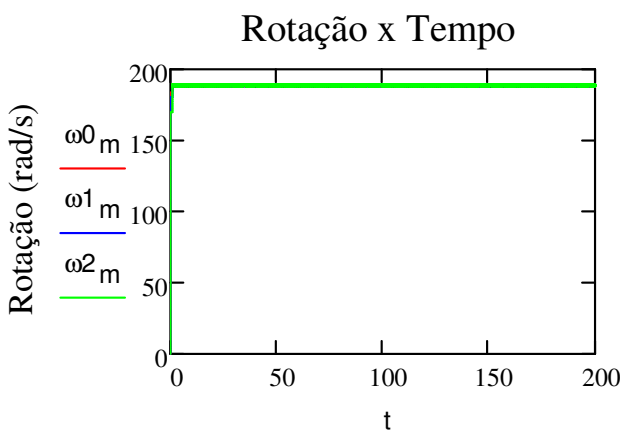

Tempo (s)

Deslocamento x Tempo

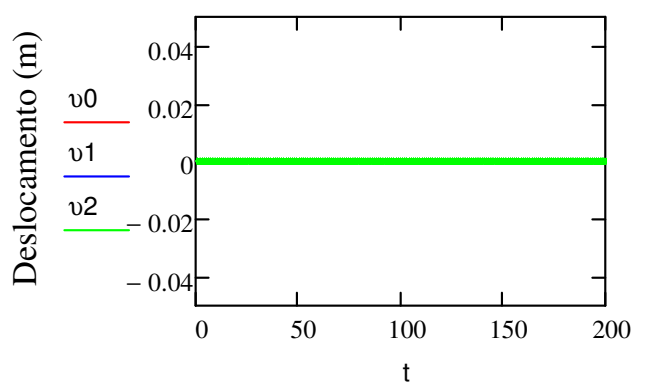

Tempo (s)

$$
\mathrm{E}_{\max }:=2.275 \cdot 10^{-4}
$$$$
\mathrm{E1}_{\text {max }}:=22.92
$$$$
\mathrm{E} 2_{\text {max }}:=91.67
$$

Rotação x Tempo

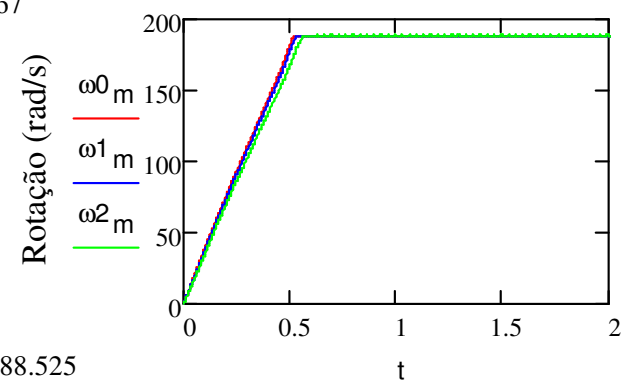

$\max \left(\omega 0_{\mathrm{m}}\right)=188.525$

$\max (\omega 1 \mathrm{~m})=188.589$

$\max \left(\omega 2_{m}\right)=188.656$

Tempo (s)

Deslocamento x Tempo

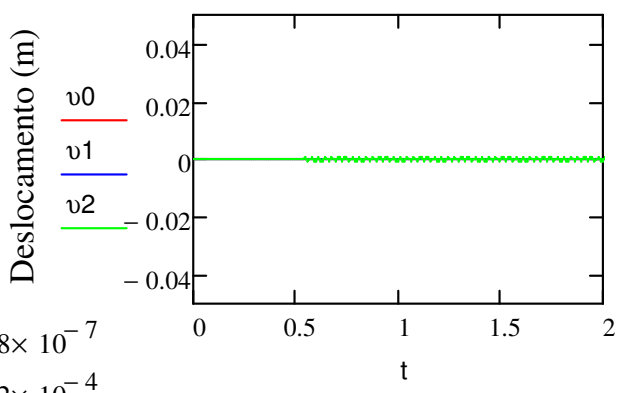

Tempo (s) $\max (v 1)=2.082 \times 10^{-4}$

$\max (v 2)=4.164 \times 10^{-4}$ 
Gráficos D.22 - Respostas da estrutura $b=0.95 \mathrm{~m}, \mathrm{~h}=0.80 \mathrm{~m}$ e $\mathrm{L}=2.0 \mathrm{~m}$

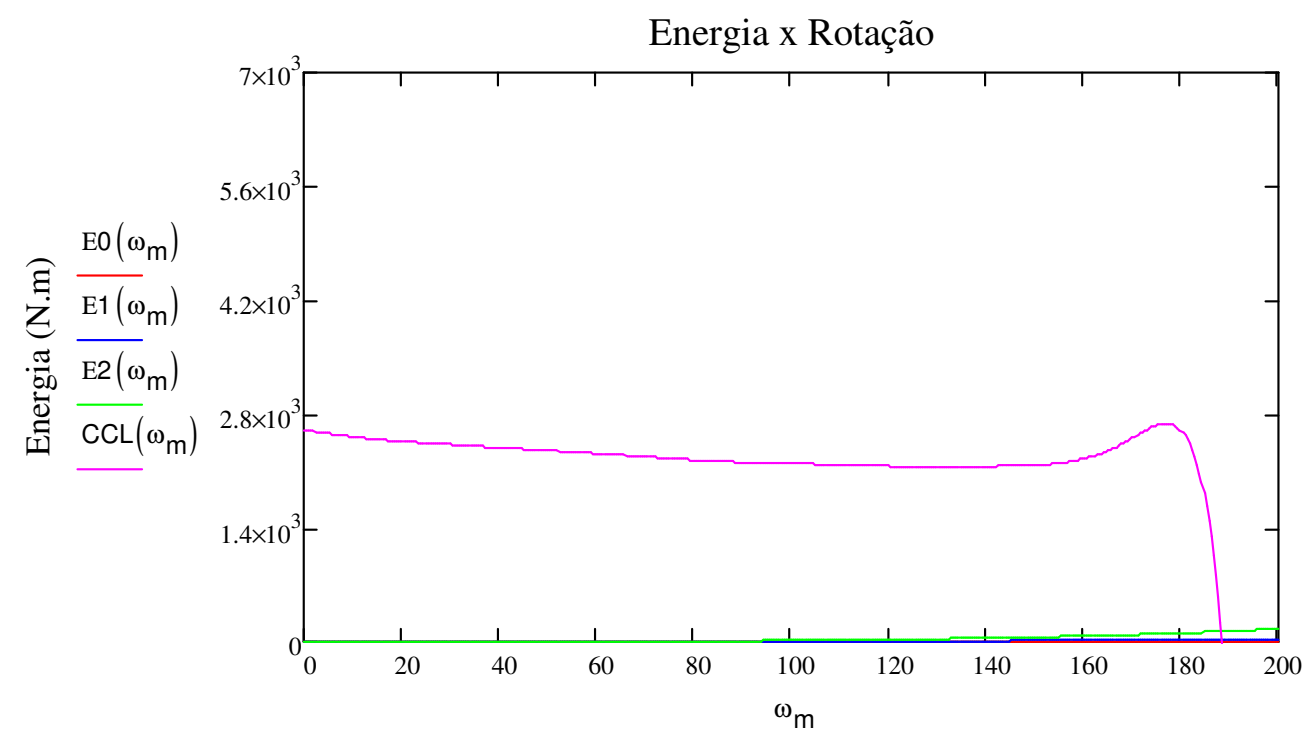

Rotação (rad/s)

_ Curva de Energia Consumida pela Estrutura para r0 : $0.034 \mathrm{~mm}$

— Curva de Energia Consumida pela Estrutura para r1 : $10.67 \mathrm{~mm}$

Curva de Energia Consumida pela Estrutura para r2: $21.34 \mathrm{~mm}$

Curva Característica Líquida do Motor (L-H)

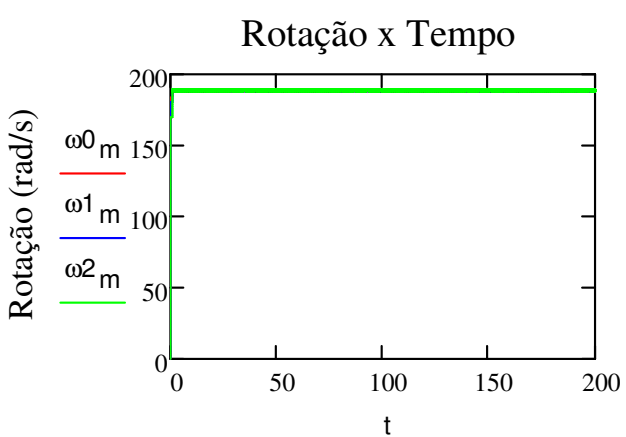

Tempo (s)

Deslocamento x Tempo

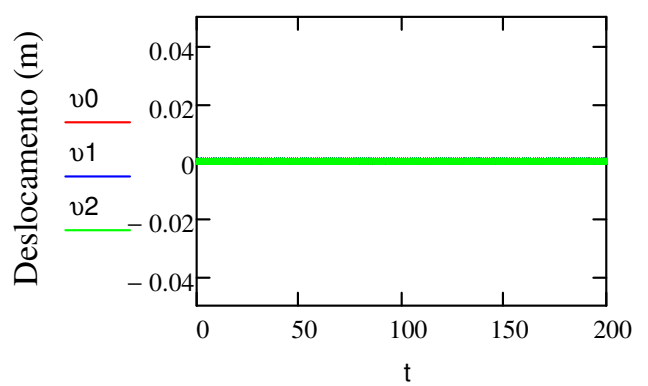

Tempo (s)

$$
\begin{aligned}
\mathrm{E}_{\text {max }} & :=3.928 \cdot 10^{-4} \\
\mathrm{E} 1_{\text {max }} & :=39.585
\end{aligned}
$$$$
\mathrm{E}_{\text {max }}:=158.345
$$

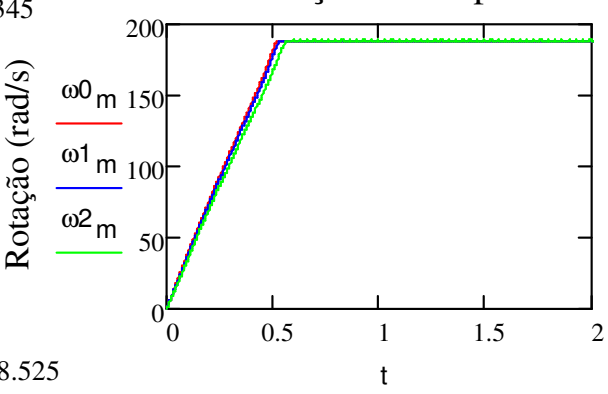

$\max \left(\omega 0_{\mathrm{m}}\right)=188.525$

$\max (\omega 1 \mathrm{~m})=188.596$

$\max \left(\omega 2_{m}\right)=188.703$

Tempo (s)

Deslocamento x Tempo

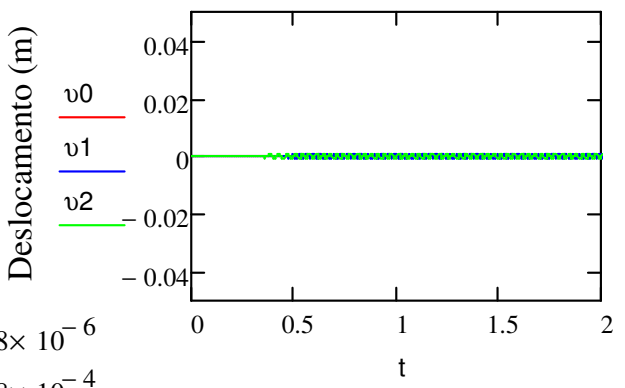

Tempo (s) 
Gráficos D.23 - Respostas da estrutura $b=0.95 \mathrm{~m}, \mathrm{~h}=0.80 \mathrm{~m}$ e $\mathrm{L}=2.5 \mathrm{~m}$

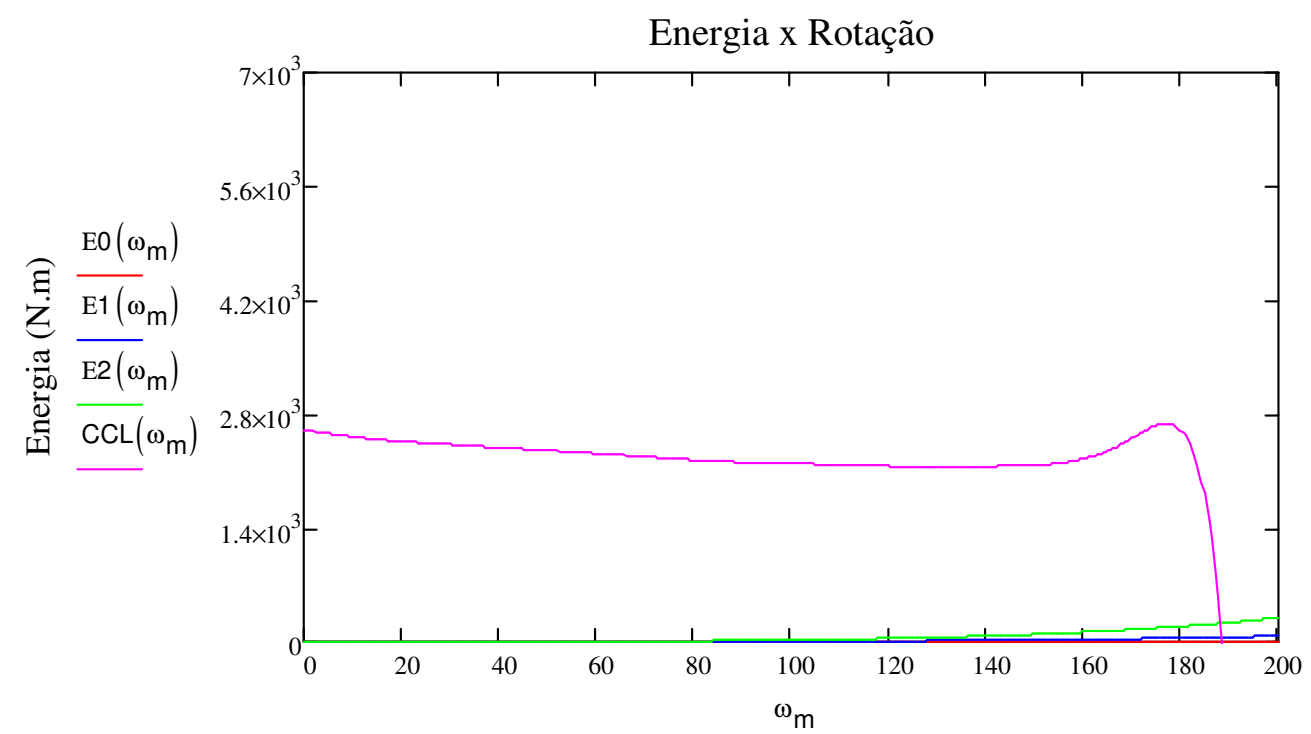

Rotação (rad/s)

_ Curva de Energia Consumida pela Estrutura para r0 : $0.034 \mathrm{~mm}$

— Curva de Energia Consumida pela Estrutura para r1 : $10.67 \mathrm{~mm}$

Curva de Energia Consumida pela Estrutura para r2: $21.34 \mathrm{~mm}$

Curva Característica Líquida do Motor (L-H)

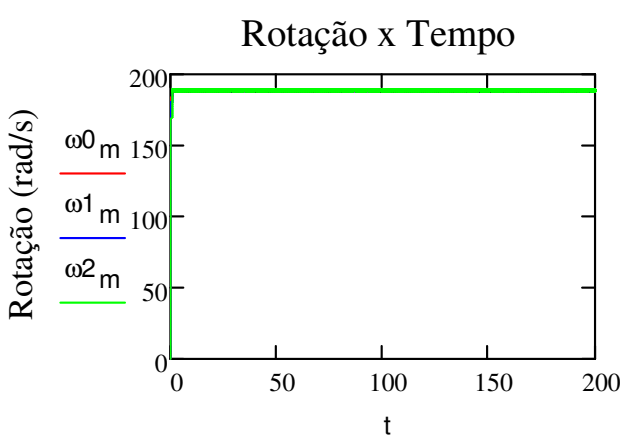

Tempo (s)

Deslocamento x Tempo

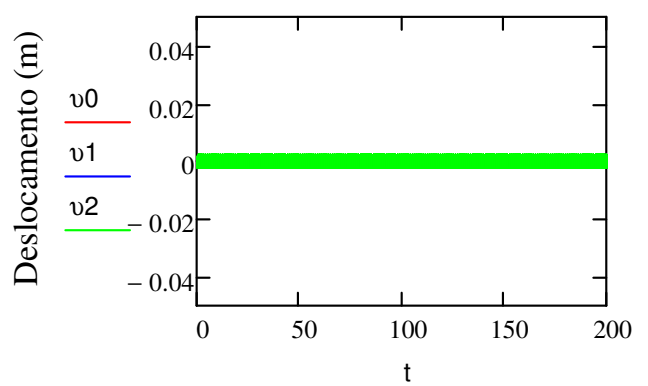

Tempo (s)

$$
\text { E0 } \max :=7.393 \cdot 10^{-4}
$$$$
\mathrm{E}_{\text {max }}:=74.503
$$$$
\mathrm{E}_{\text {max }} \text { := } 298.01
$$

Rotação x Tempo

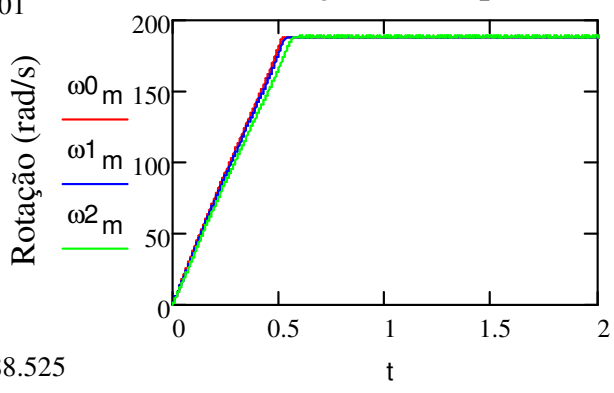

$\max \left(\omega 0_{\mathrm{m}}\right)=188.525$

$\max \left(\omega 1^{\mathrm{m}}\right)=188.623$

$\max \left(\omega 2_{m}\right)=188.822$

Tempo (s)

Deslocamento x Tempo

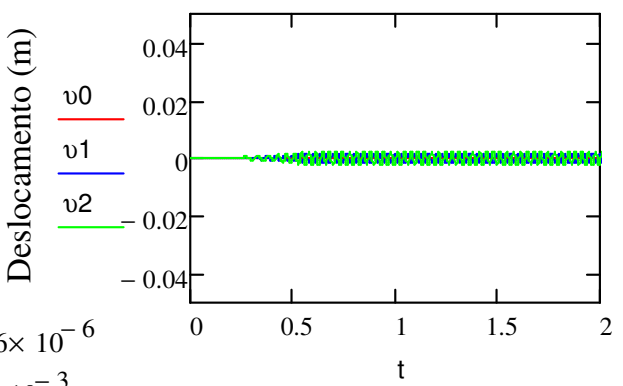

Tempo (s) 
Gráficos D.24 - Respostas da estrutura $b=0.95 \mathrm{~m}, \mathrm{~h}=0.80 \mathrm{~m}$ e $\mathrm{L}=3.0 \mathrm{~m}$

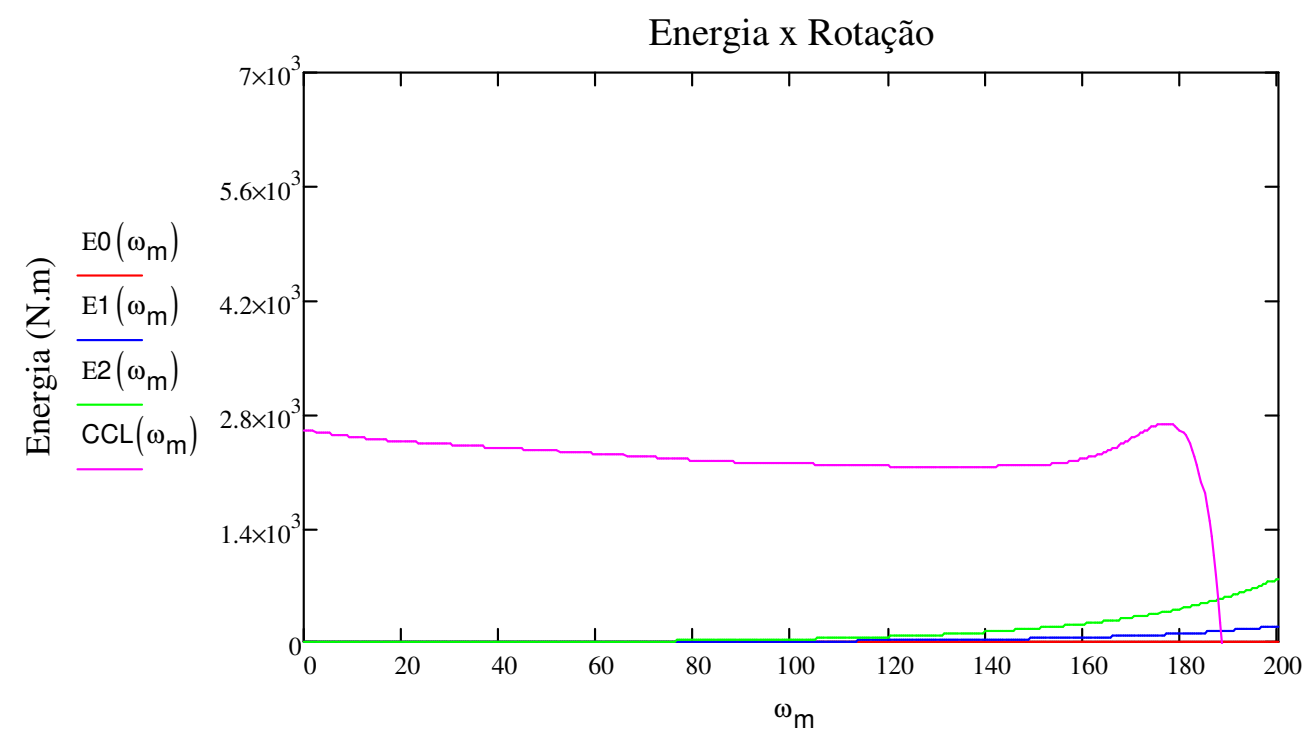

Rotação (rad/s)

— Curva de Energia Consumida pela Estrutura para r0 : $0.034 \mathrm{~mm}$

— Curva de Energia Consumida pela Estrutura para r1 : $10.67 \mathrm{~mm}$

Curva de Energia Consumida pela Estrutura para r2: $21.34 \mathrm{~mm}$

Curva Característica Líquida do Motor (L-H)

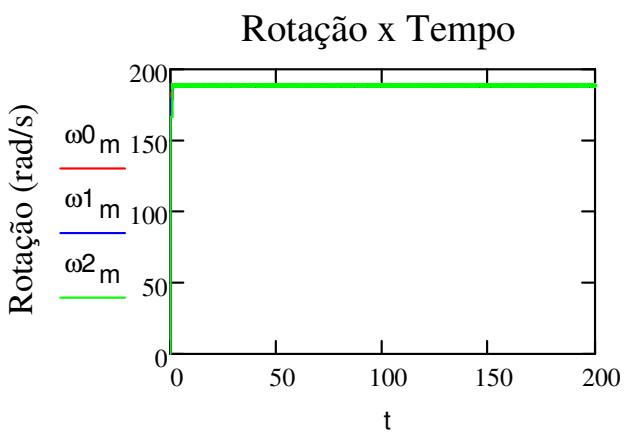

Tempo (s)

Deslocamento x Tempo

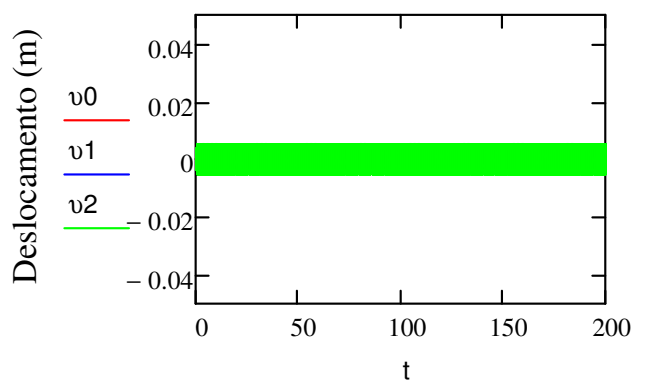

Tempo (s)

$$
\mathrm{E}_{\text {max }}:=1.937 \cdot 10^{-3}
$$$$
\mathrm{E1}_{\text {max }}:=195.221
$$$$
\text { E2 }{ }_{\text {max }}:=780.885
$$

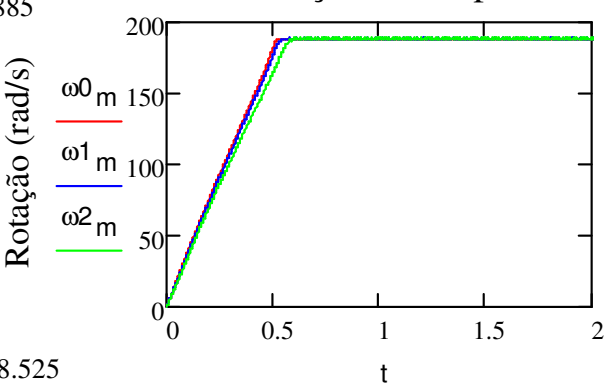

$\max \left(\omega 0_{\mathrm{m}}\right)=188.525$

$\max \left(\omega 1_{\mathrm{m}}\right)=188.672$

$\max \left(\omega 2_{m}\right)=188.972$

Tempo (s)

Deslocamento x Tempo

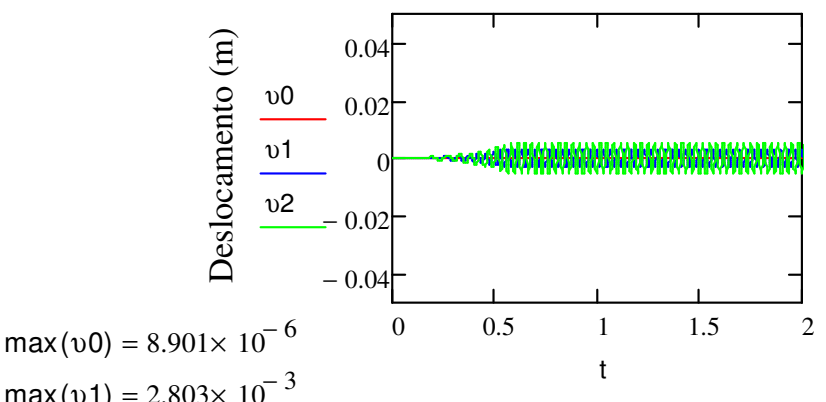

Tempo (s) $\max (v 1)=2.803 \times 10^{-3}$

$\max (v 2)=5.583 \times 10^{-3}$
Rotação x Tempo

Tempo (s) 
Gráficos D.25 - Respostas da estrutura $b=0.95 m, h=0.80 m$ e $L=3.5 m$

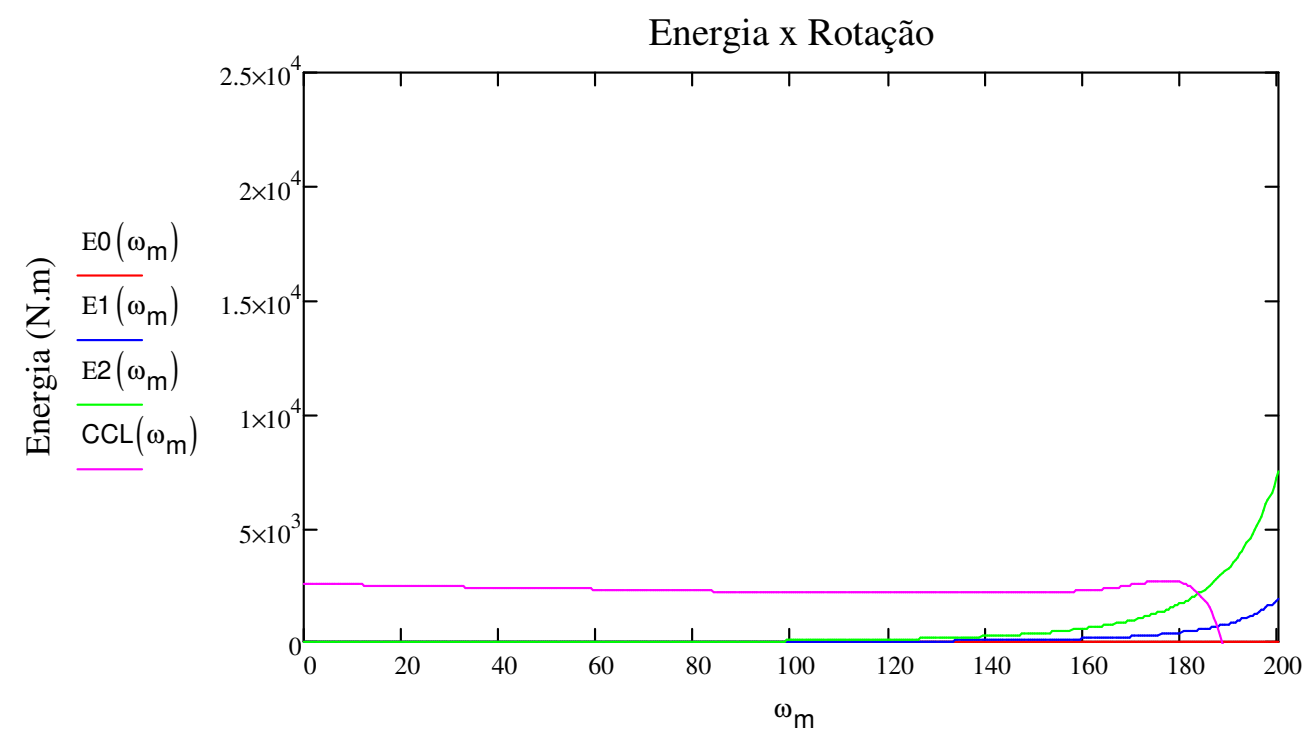

Rotação (rad/s)

— Curva de Energia Consumida pela Estrutura para r0 : $0.034 \mathrm{~mm}$

— Curva de Energia Consumida pela Estrutura para r1: $10.67 \mathrm{~mm}$

- Curva de Energia Consumida pela Estrutura para r2: $21.34 \mathrm{~mm}$

— Curva Característica Líquida do Motor (L-H)

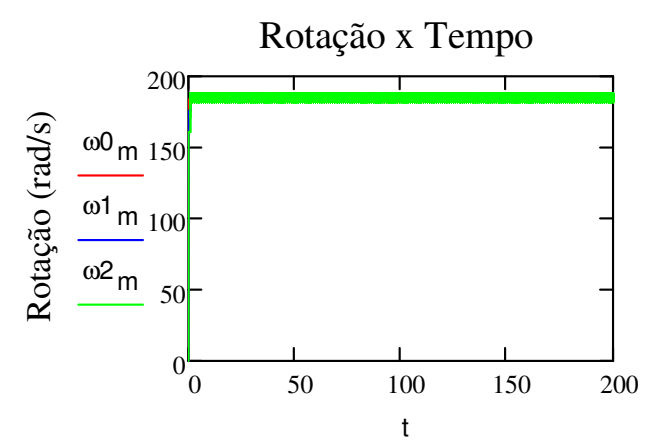

Tempo (s)

Deslocamento x Tempo

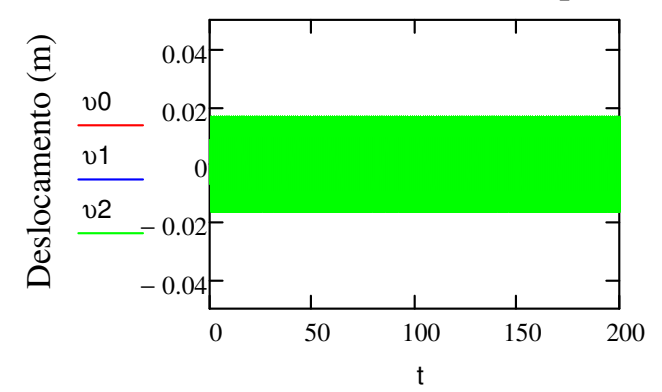

Tempo (s)

$$
\begin{aligned}
& \mathrm{E}_{\text {max }}:=0.019 \\
& \mathrm{E} 1_{\text {max }}:=1.873 \cdot 10^{3} \\
& \mathrm{E}_{\text {max }}:=7.493 \cdot 10^{3}
\end{aligned}
$$

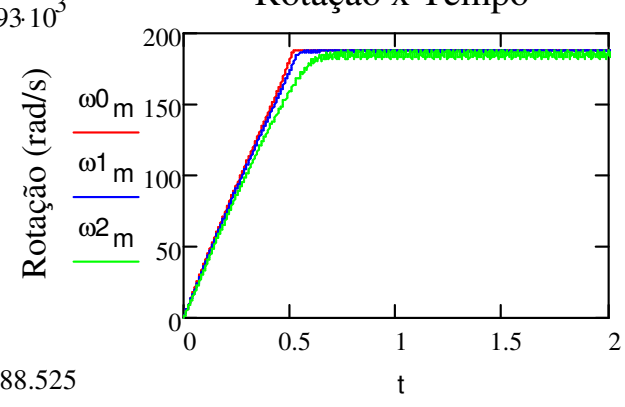

$\max \left(\omega 0_{m}\right)=188.525$

$\max (\omega 1 \mathrm{~m})=188.506$

$\max \left(\omega 2_{m}\right)=187.242$

Tempo (s)

Deslocamento x Tempo

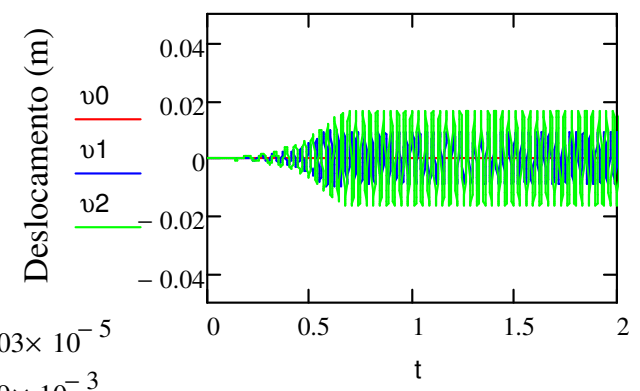

Tempo (s) $\max (v 0)=3.303 \times 10^{-5}$

$\max (v 1)=9.79 \times 10^{-3}$

$\max (v 2)=0.017$ 
Gráficos D.26 - Respostas da estrutura $b=0.95 \mathrm{~m}, \mathrm{~h}=0.80 \mathrm{~m}$ e $\mathrm{L}=4.0 \mathrm{~m}$

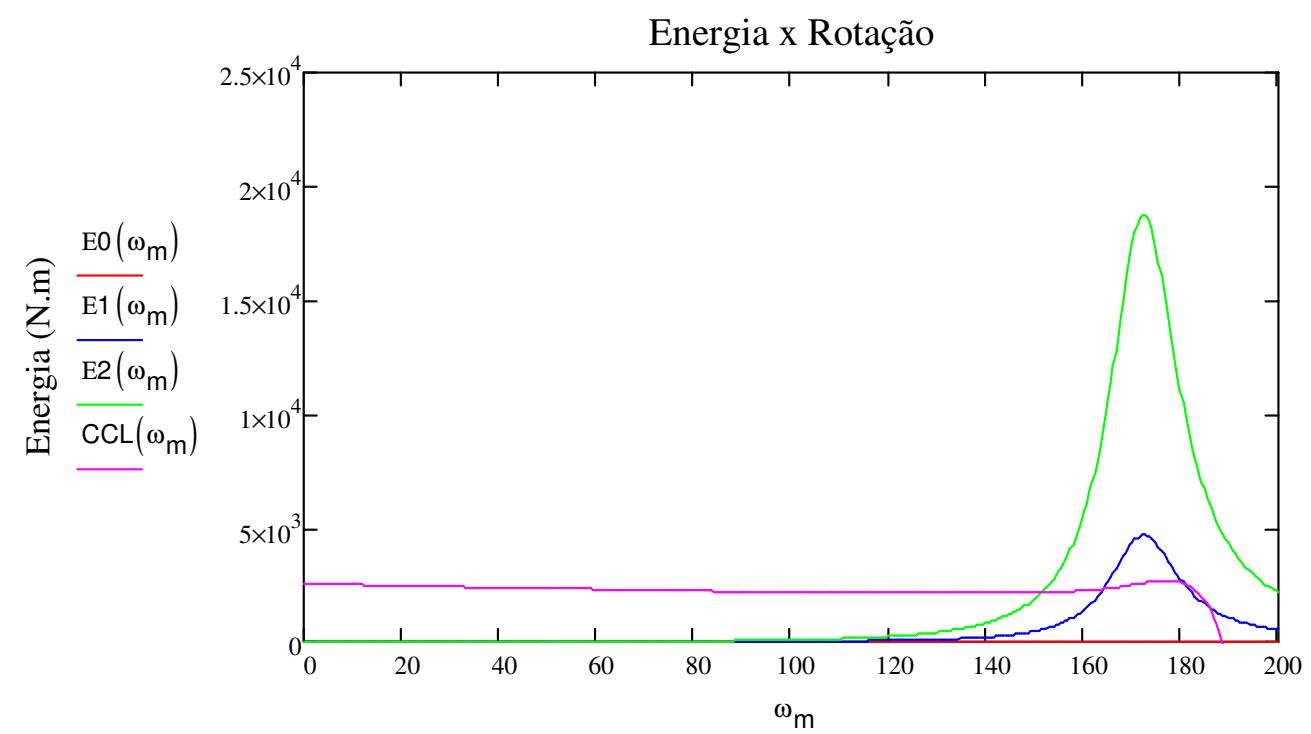

Rotação (rad/s)

— Curva de Energia Consumida pela Estrutura para r0 : $0.034 \mathrm{~mm}$

— Curva de Energia Consumida pela Estrutura para r1 : $10.67 \mathrm{~mm}$

Curva de Energia Consumida pela Estrutura para r2: $21.34 \mathrm{~mm}$ Curva Característica Líquida do Motor (L-H)

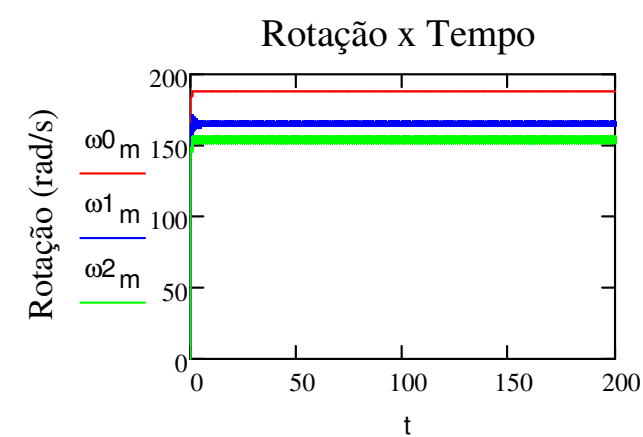

Tempo (s)

Deslocamento x Tempo

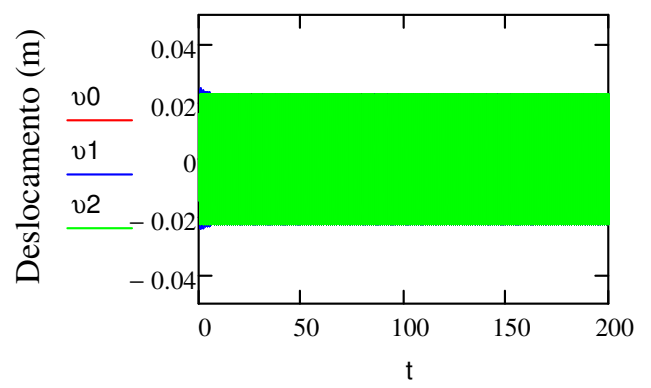

Tempo (s)

$$
\begin{aligned}
& \mathrm{E}_{\text {max }}:=0.047 \\
& \mathrm{E} 1_{\text {max }}:=4.696 \cdot 10^{3} \\
& { }^{\mathrm{E}}{ }_{\text {max }}:=1.878 \cdot 10^{4}
\end{aligned}
$$

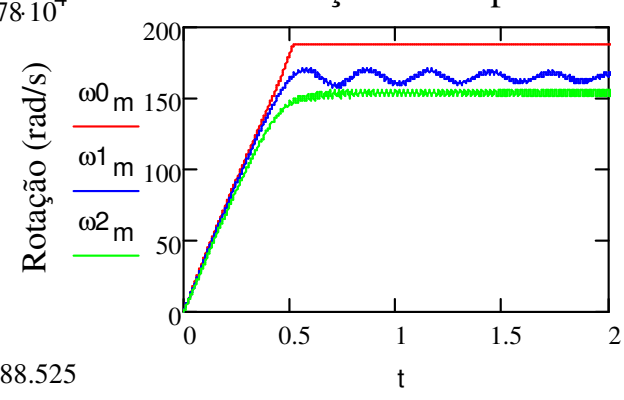

$\max \left(\omega 0_{m}\right)=188.525$

$\max (\omega 1 \mathrm{~m})=171.423$

$\max (\omega 2 \mathrm{~m})=156.51$

Tempo (s)

Deslocamento x Tempo

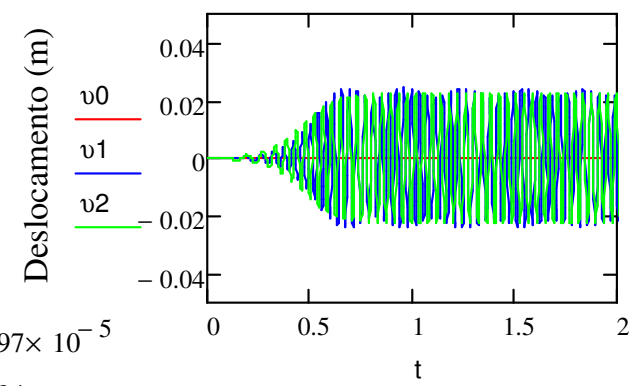

Tempo (s) 
Gráficos D.27 - Respostas da estrutura $b=0.95 m, h=0.80 m$ e $L=4.5 m$

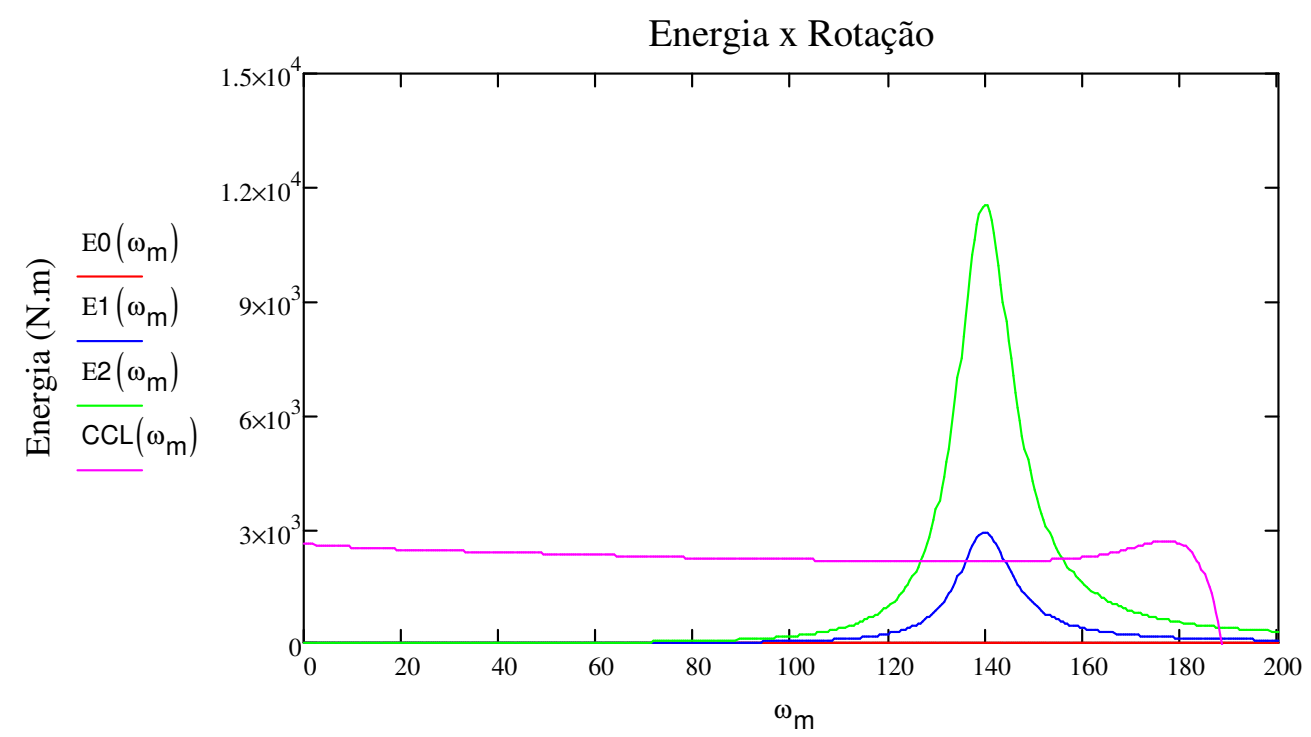

Rotação (rad/s)

— Curva de Energia Consumida pela Estrutura para r0 : $0.034 \mathrm{~mm}$

— Curva de Energia Consumida pela Estrutura para r1: $10.67 \mathrm{~mm}$

Curva de Energia Consumida pela Estrutura para r2: $21.34 \mathrm{~mm}$

— Curva Característica Líquida do Motor (L-H)

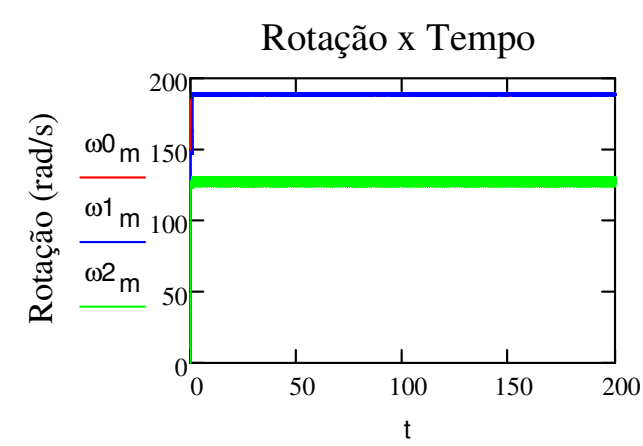

Tempo (s)

Deslocamento x Tempo

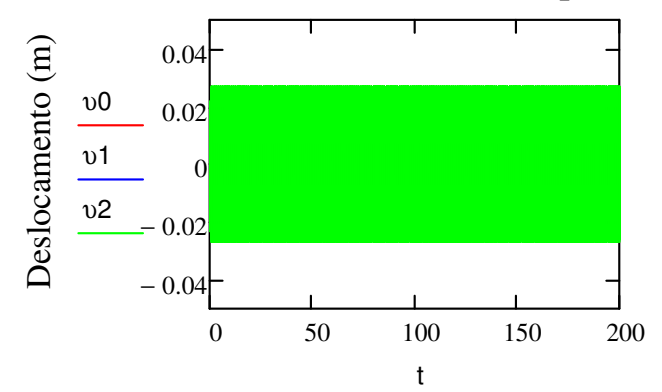

Tempo (s)

$$
\begin{aligned}
& \mathrm{E}_{\text {max }}:=0.029 \\
& \mathrm{E} 1_{\text {max }}:=2.89 \cdot 10^{3} \\
& \mathrm{E}_{\text {max }}:=1.156 \cdot 10^{4}
\end{aligned}
$$

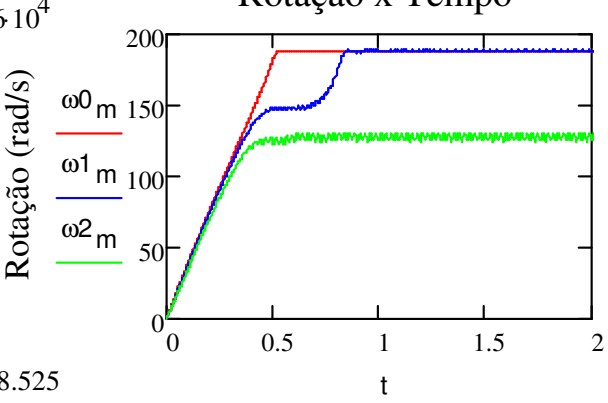

$\max \left(\omega 0_{m}\right)=188.525$

$\max \left(\omega 1^{m}\right)=189.111$

$\max (\omega 2 \mathrm{~m})=130.746$

Tempo (s)

Deslocamento x Tempo

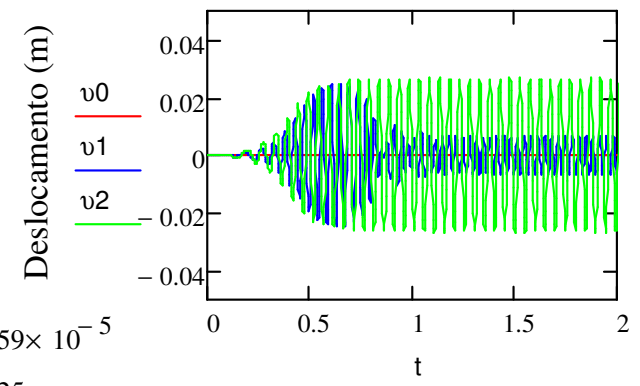

Tempo (s) 
Gráficos D.28 - Respostas da estrutura $b=0.95 \mathrm{~m}, \mathrm{~h}=0.80 \mathrm{~m}$ e $\mathrm{L}=5.0 \mathrm{~m}$

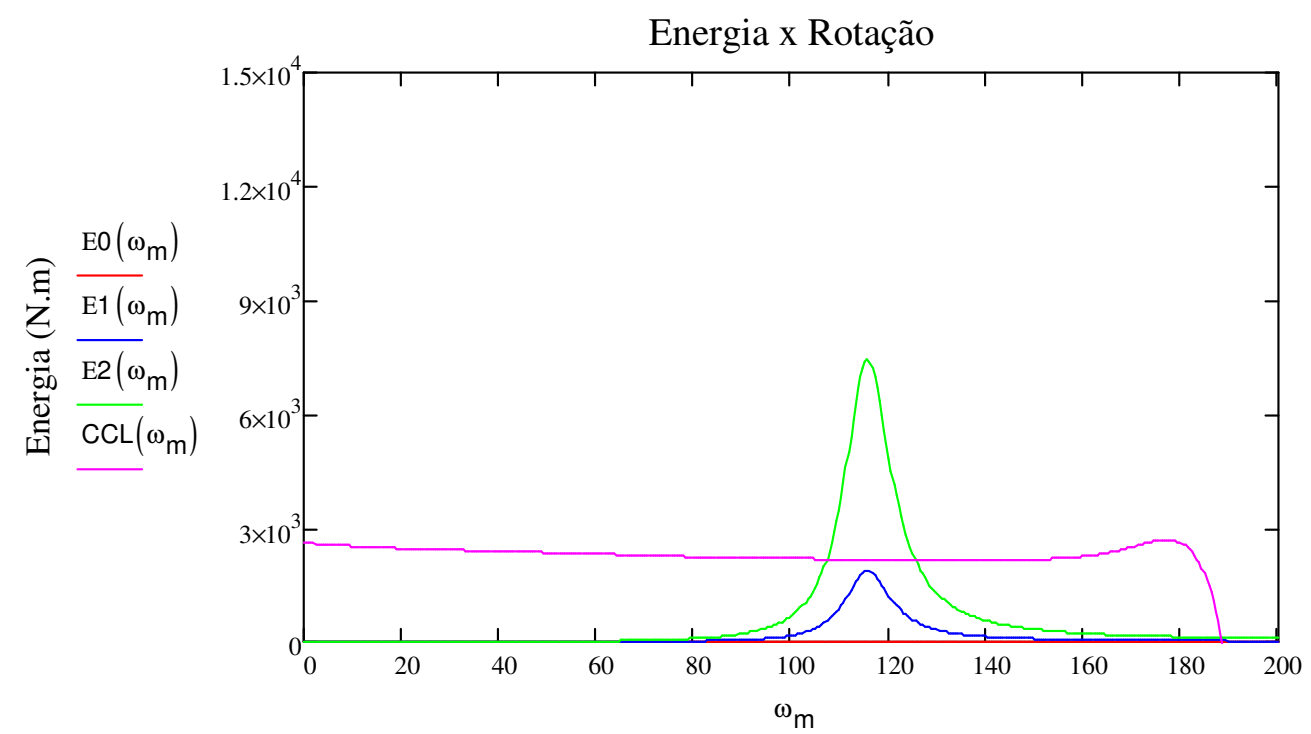

Rotação (rad/s)

_ Curva de Energia Consumida pela Estrutura para r0 : $0.034 \mathrm{~mm}$

— Curva de Energia Consumida pela Estrutura para r1: $10.67 \mathrm{~mm}$

Curva de Energia Consumida pela Estrutura para r2: $21.34 \mathrm{~mm}$ Curva Característica Líquida do Motor (L-H)

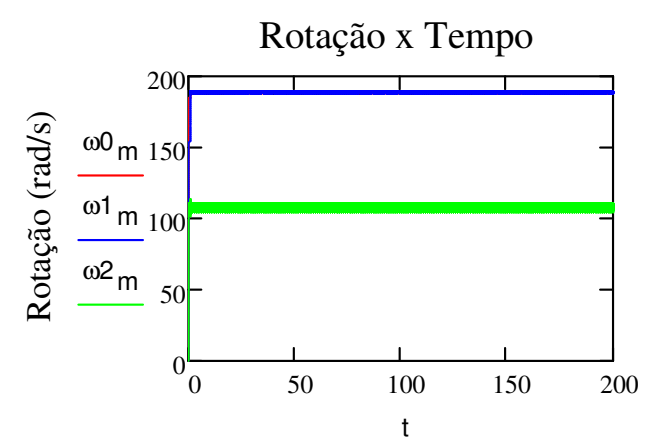

Tempo (s)

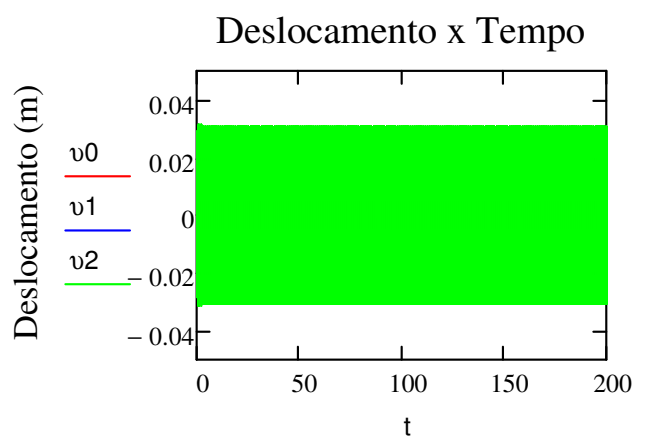

Tempo (s)

$$
\begin{aligned}
& \mathrm{E}_{\text {max }}:=0.018 \\
& \mathrm{E}_{\text {max }}:=1.861 \cdot 10^{3} \\
& { }^{\mathrm{E} 2} \text { max }
\end{aligned}
$$

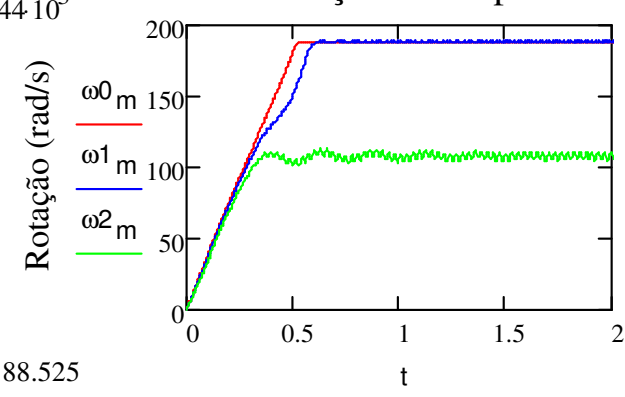

$\max \left(\omega 0_{m}\right)=188.525$

$\max \left(\omega 1^{m}\right)=188.98$

$\max \left(\omega 2_{m}\right)=112.925$

Tempo (s)

Deslocamento x Tempo

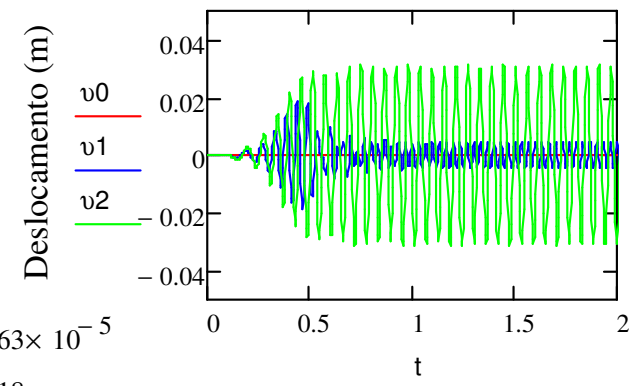

$\max (v 1)=0.018$

$\max (v 2)=0.032$

Tempo (s) 
Gráficos D.29 - Respostas da estrutura $b=0.95 m, h=0.80 m$ e $L=5.5 m$

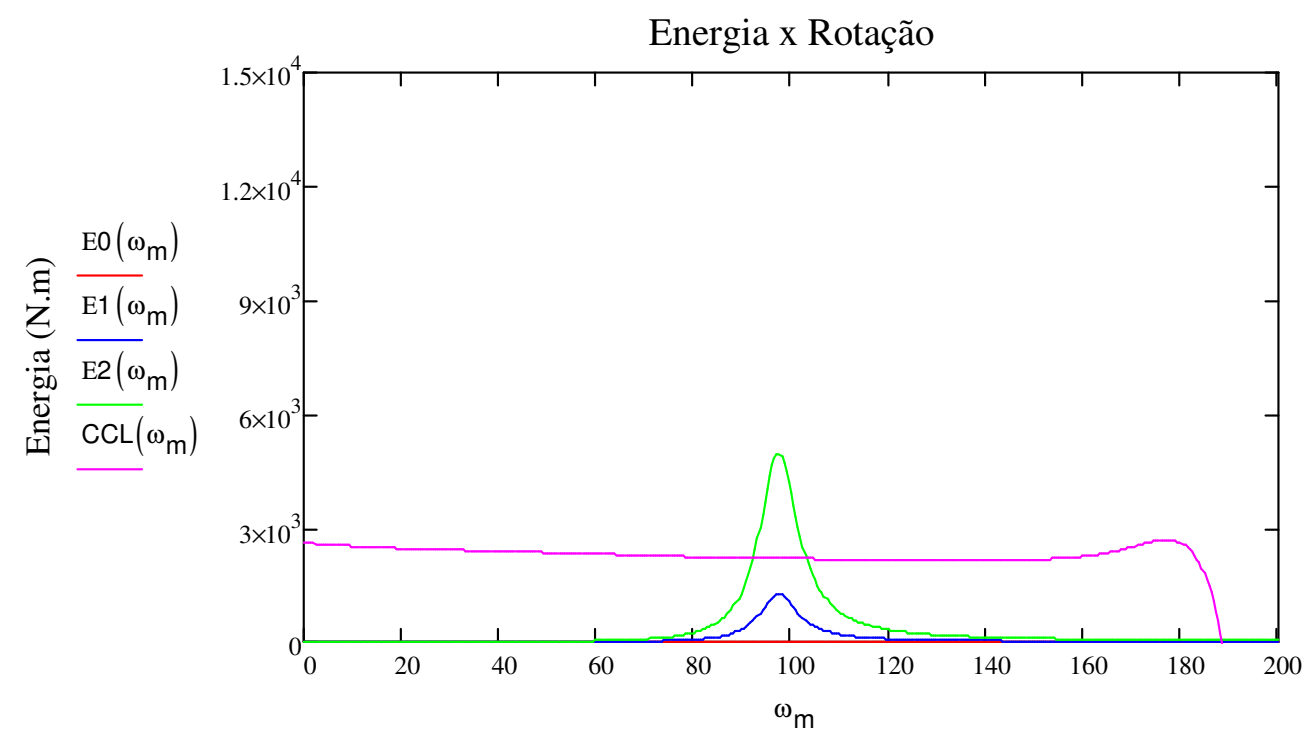

Rotação (rad/s)

— Curva de Energia Consumida pela Estrutura para r0 : $0.034 \mathrm{~mm}$

_ Curva de Energia Consumida pela Estrutura para r1 : $10.67 \mathrm{~mm}$

Curva de Energia Consumida pela Estrutura para r2: $21.34 \mathrm{~mm}$ Curva Característica Líquida do Motor (L-H)

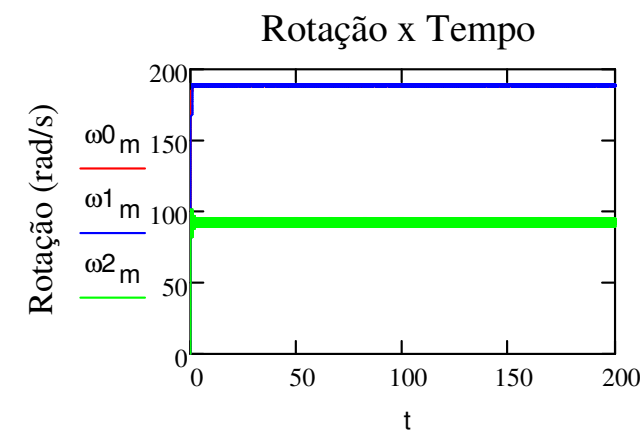

Tempo (s)

Deslocamento x Tempo

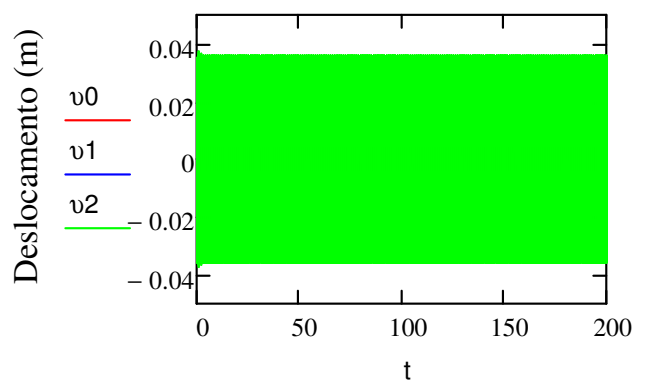

Tempo (s)

$$
\begin{aligned}
& \mathrm{E}_{\text {max }}:=0.012 \\
& \mathrm{E}_{\text {max }}:=1.243 \cdot 10^{3} \\
& \mathrm{E}_{\text {max }}:=4.971 \cdot 10^{3}
\end{aligned}
$$

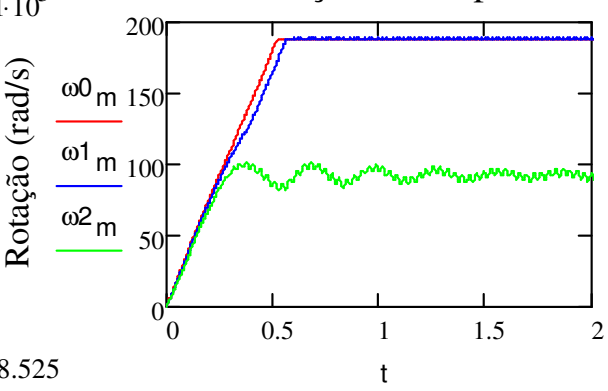

$\max \left(\omega 0_{\mathrm{m}}\right)=188.525$

$\max \left(\omega 1^{\mathrm{m}}\right)=188.801$

$\max \left(\omega 2_{m}\right)=101.782$

Tempo (s)

Deslocamento x Tempo

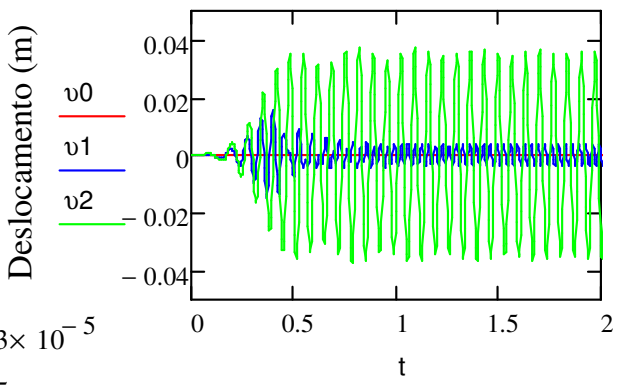

$\max (v 1)=0.015$

$\max (v 2)=0.037$

Tempo (s) 
Gráficos D.30 - Respostas da estrutura $b=0.95 \mathrm{~m}, \mathrm{~h}=0.80 \mathrm{~m}$ e $\mathrm{L}=6.0 \mathrm{~m}$

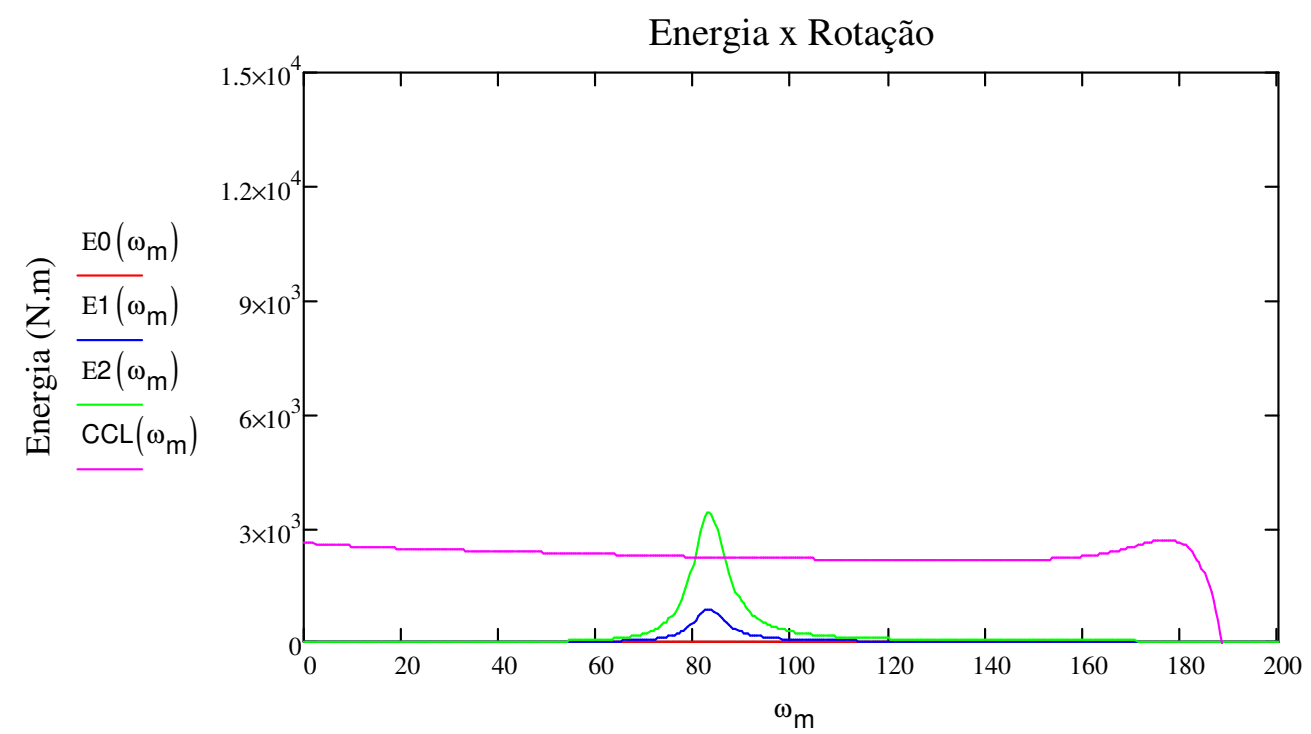

Rotação (rad/s)

— Curva de Energia Consumida pela Estrutura para r0 : $0.034 \mathrm{~mm}$

— Curva de Energia Consumida pela Estrutura para r1: $10.67 \mathrm{~mm}$

Curva de Energia Consumida pela Estrutura para r2: $21.34 \mathrm{~mm}$

Curva Característica Líquida do Motor (L-H)

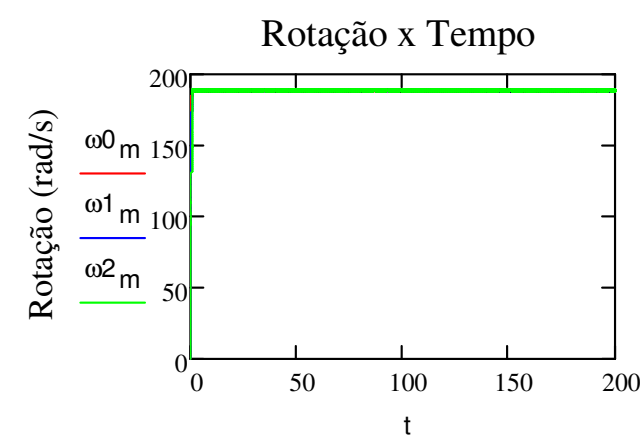

Tempo (s)

Deslocamento x Tempo

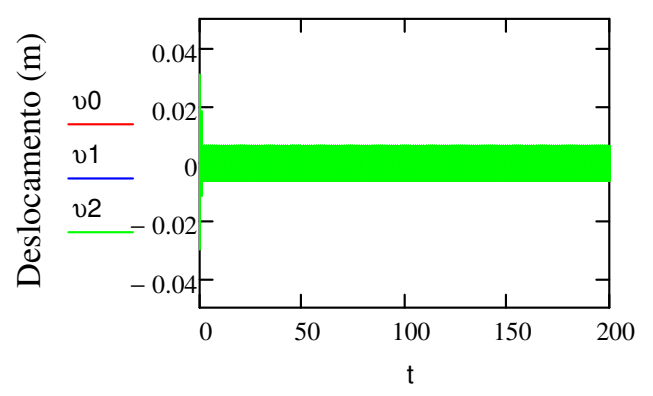

Tempo (s)

$$
\mathrm{E}_{\text {max }}:=8.495 \cdot 10^{-3}
$$$$
\mathrm{E}_{\text {max }}:=856.24
$$$$
\mathrm{E}_{\text {max }}:=3.425 \cdot 10^{3}
$$

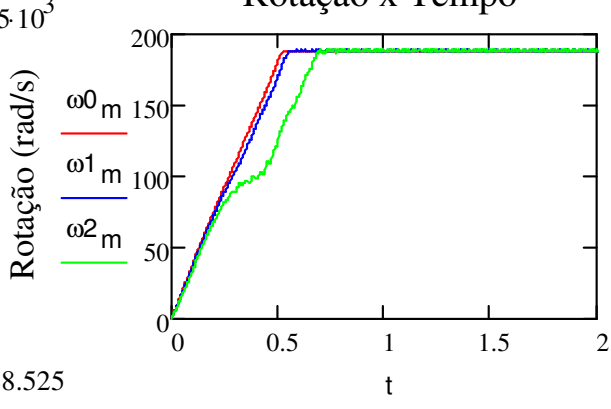

$\max \left(\omega 0_{m}\right)=188.525$

$\max \left(\omega 1_{\mathrm{m}}\right)=188.77$

$\max \left(\omega 2_{m}\right)=189.505$

Tempo (s)

Deslocamento x Tempo

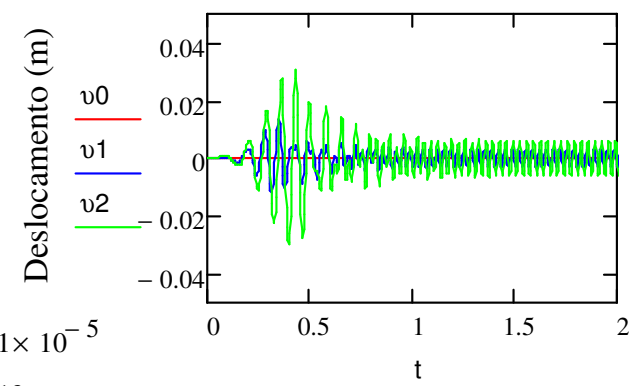

Tempo (s) 


\section{ANEXO A - CARACTERÍSTICAS MECÂNICAS DO MOTOR WEG}

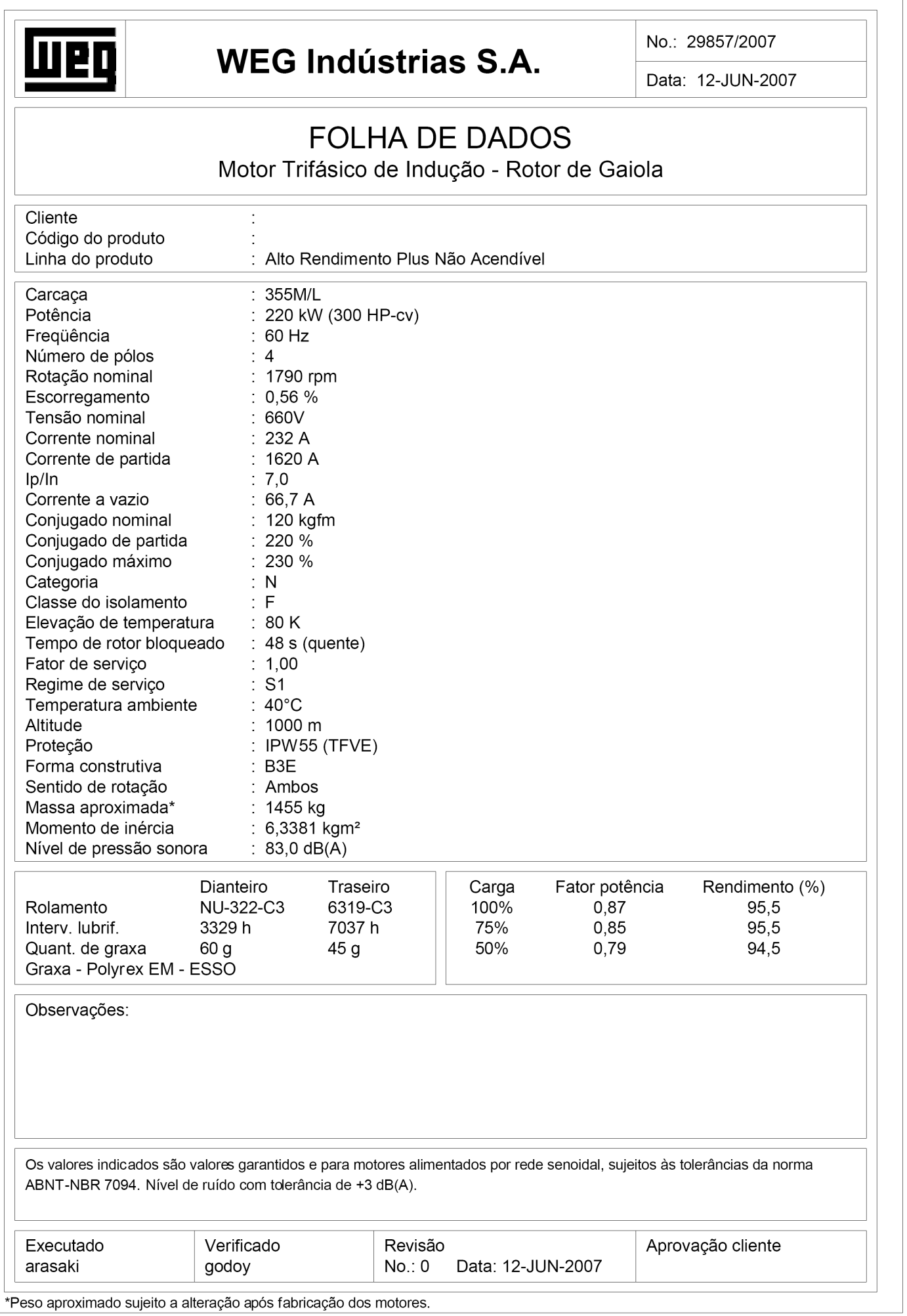




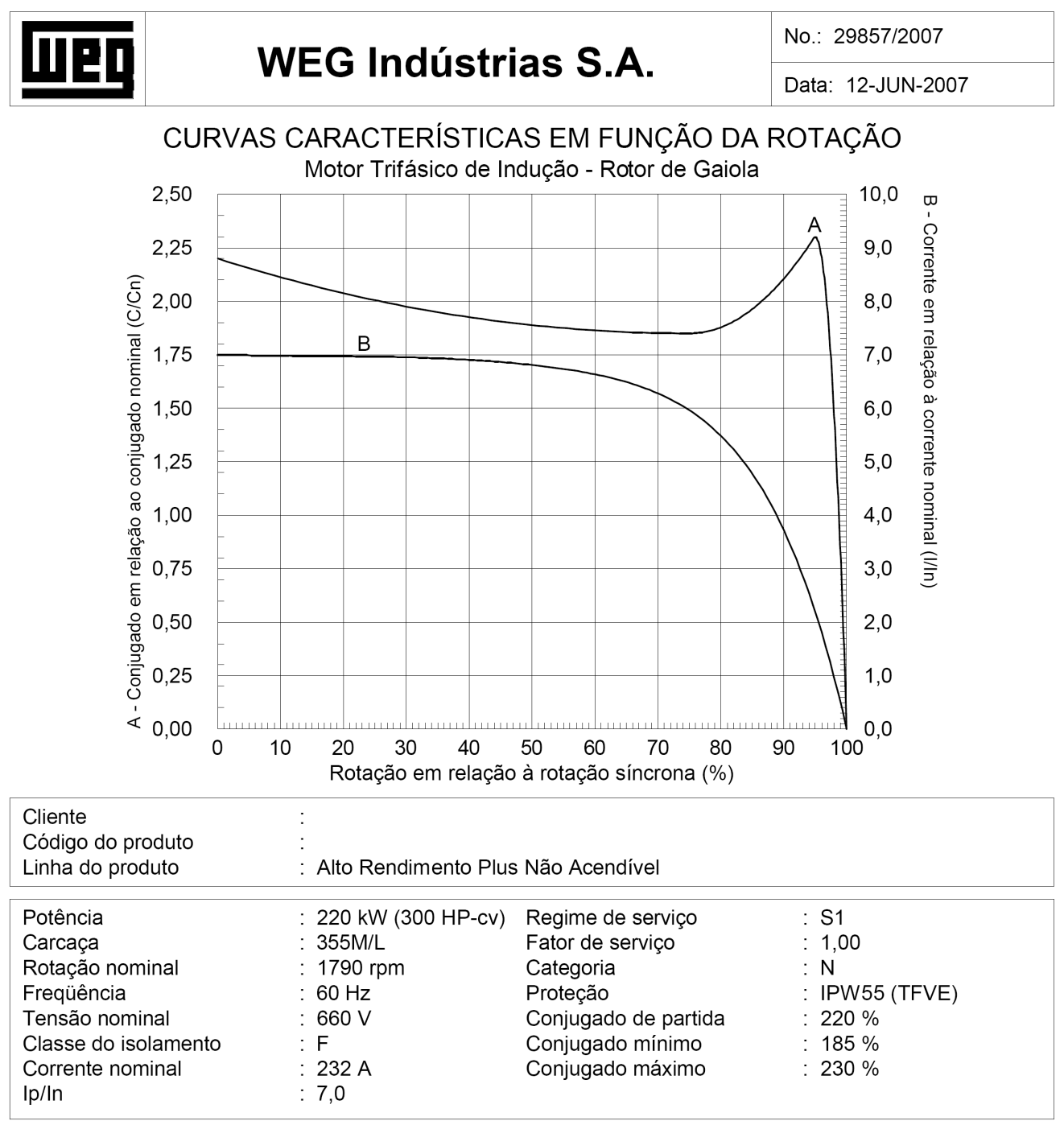

Observações:

Os valores indicados são valores garantidos e para motores alimentados por rede senoidal, sujeitos às tolerâncias da norma ABNT-NBR 7094.

\begin{tabular}{l|l|l|l|}
$\begin{array}{l}\text { Executado } \\
\text { arasaki }\end{array}$ & $\begin{array}{l}\text { Verificado } \\
\text { godoy }\end{array}$ & $\begin{array}{l}\text { Revisão } \\
\text { No.: 0 Data: 12-JUN-2007 }\end{array}$ & Aprovação cliente \\
\hline
\end{tabular}


Motor trifísico IP55

Motor trifísico de Alto Rendimento Plus

Motor trifísicico não acendlivel

Motor trifísico Dahlander IP55 (duas velocidades)
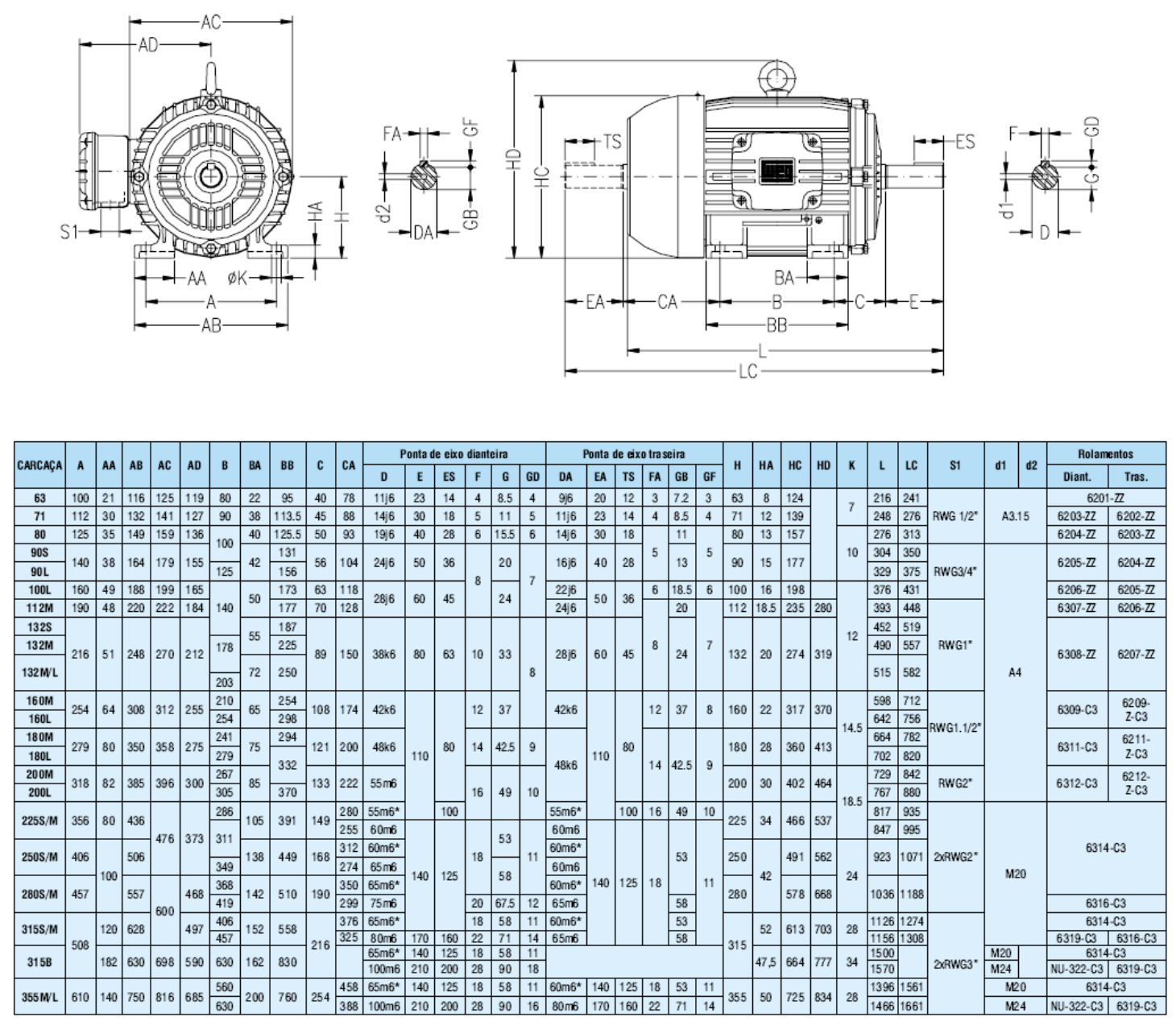

* Dimensóes da ponta de eixo para motores em II pólos.

- A partir da carcaça 160 , inclusive, os rolamentos sáo com folga radial C3.

- Nas carcaças acima de $200 \mathrm{~S} / \mathrm{M}$ a medica $\mathrm{H}$ tem tolerancia $-1 \mathrm{~mm}$.

- Dimensỏes são normalizadas pela norma NBR 5432, sujeitas a alteração sem aviso prévio. 